\title{
Tension in New Zealand's public hospitals: performance effects of sharpening incentives
}

\author{
By
}

Adrian Slack

A thesis submitted to the Victoria University of Wellington in fulfilment of the requirements for the degree of

Doctor of Philosophy in Economics

Victoria University of Wellington

2011 



\section{Abstract}

New Zealand's health sector reforms in the mid-1990s introduced corporate institutions and market disciplines to public hospitals. Yet the reorganisation of New Zealand's public hospitals into Crown Health Enterprises (CHEs) led to severe criticisms. Ultimately the CHEs were replaced with non-profit Hospital and Health Services.

This thesis focuses on three major criticisms of the CHEs. We use game theory to provide a formal and novel analysis of interactions that could cause an organisation's performance to differ markedly from the reformers' expectations. The analysis explains how a stylised set of reforms could fail to achieve their objectives.

Chapter 2 analyses public hospital throughput data over the reform period. We find that the CHE reforms were independently associated with an increase in hospitals' treatment costs. This chapter motivates the theoretical analyses of the three criticisms of the CHEs.

We structure the theoretic analysis using an organisational hierarchy with four actors: a funder, an (hospital) administrator, a (medical) specialist and a (health) consumer.

The first criticism was that CHE Boards paid bonuses despite managers failing to achieve performance targets. Chapter 3 examines when a funder may want to revise the budget of an organisation and to pay the administrator a bonus despite failing to meet a target. We introduce three features of the CHE reforms that conventional soft budget constraint models partly or entirely neglect: funder bargaining power, revisable targets and performance bonuses. A flexible budget constraint paired with bonuses can be efficient in the light of uncertainty. 
The second criticism was that costs escalated despite strong managerial incentives for cost control. Chapter 4 argues that such incentives could disrupt trust in an organisation. We show that sharpening the administrator's incentives for cost control can create a misalignment between the administrator and the specialist and cause costs to escalate. Our result, that incentivising a measurable dimension of performance can worsen performance of that same task, contrasts with the conventional game-theoretic literature.

The third criticism was that the reforms let doctors manipulate managers, resulting in inefficiency. The first model of Chapter 5 shows that an administrator might want to encourage a specialist to influence public opinion. We modify the first model to reflect a feature of the reforms: managerial efforts aimed at improving the organisation's operation. The administrator can damage a whistle-blower's credibility, to the detriment of specialists and patients. Both models give original insights into how the reforms could let an administrator take advantage of his role. In this multi-layered model, the administrator may intentionally reduce communication.

The CHE reformers expected performance incentives to flow through a corporate structure to improve efficiency. Rather than a cascade of beneficial incentives, incomplete contracts could cause unintentional negative interactions. Tension and perverse incentives could have caused costs to rise, necessitating budget revisions and additional bonus payments, while permitting administrators to silence whistle-blowers. This research shows how complex organisations that rely on soft information can benefit from systems that enhance trust and collaboration, and may be harmed by unhealthy tension. 


\section{Acknowledgements}

- My supervisors. Paul Calcott, for his dedication, reminding me that pages of math may make the model work but make the reader work too. And for improving my posture. Jack Robles, for your thoroughness, insights and suggestions on scope.

- Chirok Han and Malathi Velamuri for your guidance on my empirical analysis, and for your enviable skill in explaining how to make a complex problem simple.

- Chris Lewis (NZHIS) for his advice on the NMDS data used in my empirical analysis.

- The School of Economics and Finance for its assistance in purchasing the NMDS data and the opportunity to be part of its lecturing team.

- BERL for its flexibility around work commitments and financial support through the Philpott-BERL Scholarship.

- My family; particularly my father, Roy, for his meticulous review.

- Julie, who supported me throughout, whether I was at home or at school, and could see the point across the many drafts. Sometimes, more is less. 


\section{Table of Contents}

\begin{tabular}{lll}
\hline Abstract & i
\end{tabular}

\begin{tabular}{|ll}
\hline Acknowledgements & iii
\end{tabular}

$\begin{array}{ll}\text { Table of Contents } & \text { v }\end{array}$

List of Tables $\quad$ ix

List of Figures $\quad$ xi

\begin{tabular}{lll}
\hline & Introduction & 1
\end{tabular}

1.1 Organisation of the thesis . . . . . . . . . . . . . . 2

1.2 Background literature . . . . . . . . . . . . . . . . . . . . . . . . . . 4

2 Empirical investigation: hospital admission and cost data, 1988-1995 13

2.1 Introduction . . . . . . . . . . . . . . . . . . . . . 13

2.2 Background . . . . . . . . . . . . . . . . . . . . . . . . 14

2.3 Hospital admission and treatment cost data $\quad . \quad$. . . . . . . . . . . . . . 15

$2.3 .1 \quad$ Data cleaning and filtering . . . . . . . . . . . . . . . . . . . 18

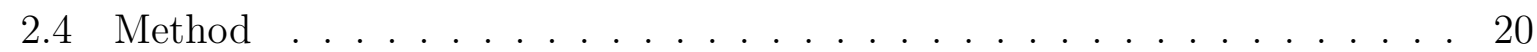

2.5 Results . . . . . . . . . . . . . . . . . . . . . . . 26

2.5 .1 Summary statistics . . . . . . . . . . . . . . . 26

2.5 .2 Econometric analysis . . . . . . . . . . . . . . . . . 36

2.6 Discussion $\ldots \ldots \ldots \ldots \ldots$ 
$\begin{array}{|ccc|}3 & \text { Designing delegation incentives in an uncertain environment } & 49\end{array}$

$3.1 \quad$ Introduction . . . . . . . . . . . . . . . . . . . . . . . . . . . . . . . 49

3.2 Delegation and performance incentives in CHEs $\ldots \ldots \ldots \ldots$

3.3 Literature $\ldots \ldots \ldots \ldots \ldots$. . . . . . . . . . . . . . . . . . 51

3.3.1 Delegation and authority in organisational hierarchies . . . . . . . 51

$3.3 .2 \quad$ The soft budget constraint syndrome . . . . . . . . . . . . 53

3.4 Budget revision and bonuses: an alternative to conventional SBC models . 55

3.4 .1 Play of the game . . . . . . . . . . . . . . . . . 56

$3.4 .2 \quad$ A hard budget constraint regime . . . . . . . . . . . 60

$3.4 .3 \quad$ A flexible budget constraint regime . . . . . . . . . . . . . . . . 64

3.4 .4 Outcomes of the game $\ldots \ldots \ldots \ldots$

$3.5 \quad$ Discussion $\ldots \ldots \ldots \ldots$. . . . . . . . . . . . . . . . . . . . . . . . 69

\begin{tabular}{|lll}
\hline & Incentive congruence, credibility and communication & 71
\end{tabular}

4.1 Introduction . . . . . . . . . . . . . . . . . . . . . . . . . . . . . 71

4.2 Managers' incentives and the CHE reforms . . . . . . . . . . . . . . 73

4.3 Literature . . . . . . . . . . . . . . . . . . . . . . . . . . . . . . 77

4.3 .1 Undesirable performance effects of sharp incentives . . . . . . . 77

4.3 .2 New Zealand's CHE reforms . . . . . . . . . . . . . . . . . . 80

$4.3 .3 \quad$ Modelling concepts used in this chapter. . . . . . . . . . . . . . . 81

4.4 Cheap talk: incentive congruence and credibility . . . . . . . . . . . . 84

4.4 .1 A motivating example of harmful cost-cutting incentives . . . . . 84

4.4 .2 Play of the game . . . . . . . . . . . . . . . . 86

$4.4 .3 \quad$ Bayesian Nash Equilibria . . . . . . . . . . . . . . . . . . . 90

$4.4 .4 \quad$ Neologism-proof Bayesian Nash equilibrium outcomes . . . . . . . . 94

4.4 .5 Sharp incentives . . . . . . . . . . . . . . . . . . . . . . 104

4.5 Discussion $\ldots \ldots \ldots \ldots$ 


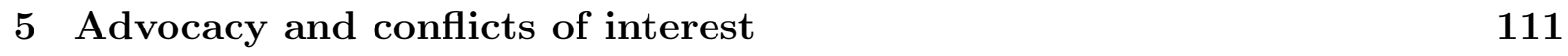

5.1 Introduction . . . . . . . . . . . . . . . . . . . . . 111

5.2 Background to the patient-doctor relationship in the CHE reforms . . . . . 113

5.3 Conflicts of interest and credibility . . . . . . . . . . . . . . . . 114

5.3 .1 Play of the game . . . . . . . . . . . . . . . 115

5.3 .2 Why setting wages can turn signals on and off . . . . . . . . . . 118

$5.3 .3 \quad$ Plausible equilibria with neologism-proof subgames . . . . . . . . . 122

5.3 .4 Results . . . . . . . . . . . . . . . . . . . . . . . . . . . . 129

5.4 Managerial effort and public expectations . . . . . . . . . . . . . . . 130

5.4 .1 Play of the game . . . . . . . . . . . . . . . . 131

5.4 .2 Solving the game . . . . . . . . . . . . . . 133

5.4 .3 Results . . . . . . . . . . . . . . . . . . . . 138

5.5 Discussion . . . . . . . . . . . . . . . . . . . . . 139

$\begin{array}{lll}6 & \text { Conclusion } & 141\end{array}$

\begin{tabular}{ll}
\hline Bibliography & 147
\end{tabular}

$\begin{array}{ll}\text { Glossary } & 155\end{array}$ 
\begin{tabular}{lr}
\hline Appendices & 159
\end{tabular}

A.1 Appendices to Chapter 2 . . . . . . . . . . . . . . . . . . . . . . 160

A.1.1 Weighted Inlier Equivalent Separations methodology . . . . . . . 160

A.1.2 NMDS records excluded from analytical data set. . . . . . . . . . 163

A.1.3 Tabulation of regression statistics . . . . . . . . . . . . . 166

A.1.4 Supplementary analysis excluding outliers . . . . . . . . . . . . 167

A.1.5 Supplementary analysis censoring transition period . . . . . . . . 171

A.2 Appendices to Chapter 3 . . . . . . . . . . . . . . . . . . . . . . . 174

A.2.1 Delegation model . . . . . . . . . . . . . . . . . . . . . . . 174

A.2.2 Soft budget constraint model . . . . . . . . . . . . . . . 183

A.3 Appendices to Chapter 4 . . . . . . . . . . . . . . . . . . . . . . . 190

A.3.1 Proof of the uniqueness of an NPE outcome . . . . . . . . . . 190

A.3.2 Example of NPE outcomes . . . . . . . . . . . . . . . . . . . . 194

A.4 Appendices to Chapter 5 . . . . . . . . . . . . . . . . . . . . . . . 198

A.4.1 Application of neologism-proofness with continuous choice variables 198

A.4.2 The minimum wage and the signalling wage . . . . . . . . . . 202 


\section{List of Tables}

$2.1 \quad$ Data excluded using NZHIS filters and due to a private procedure . . . . . 20

2.2 Variable definitions . . . . . . . . . . . . . . . . . . . 25

2.3 Summary of regression model variables . . . . . . . . . . . . . . . . . . . 25

2.4 Number of observations . . . . . . . . . . . . . . . . . . . . . . . 27

2.5 Cost weight statistics (filtered data set, $n=6,370,748$ ) . . . . . . . . . . . . 28

$2.6 \quad$ Age statistics (filtered data set, $n=6,370,748$ ) $\ldots \ldots$. . . . . . . . . 28

2.7 Change in average cost weight and age over the reform period . . . . . . . 29

2.8 Summary of regression model results $\ldots \ldots \ldots$

4.1 Utilities for the specialist and administrator . . . . . . . . . . . . . . . 85

$4.2 \quad S^{\prime}$ s preferred choices $\ldots \ldots \ldots \ldots$. . . . . . . . . . . . . . . . 92

4.3 Conditions for $T_{0}$ to be consistent with a BNE outcome . . . . . . . . . . . 93

4.4 Conditions for deviating from a potential BNE outcome with $\left|T_{0}\right|=2$. . . 96

4.5 Conditions for deviating from a potential BNE outcome with $\left|T_{0}\right|=1$. . . 97

4.6 Conditions for $T_{0}$ to be consistent with a NPE outcome . . . . . . . . . . . 99

4.7 Illustrative example of NPE outcome existence . . . . . . . . . . . . . . . 103 
5.1 The specialist's choice . . . . . . . . . . . . . . . . . . . . 119

5.2 Conditions for $Y_{0}$ to be consistent with a PBE outcome . . . . . . . . . . . 122

5.3 Conditions for deviating from a potential PBE outcome with $\left|Y_{0}\right|=1$. . . 123

5.4 Conditions for $Y_{0}$ to be consistent with a potential NPE outcome . . . . . 124

5.5 The probability of reappointment in case 3 given effort and the wage . . . 137

A.1 Records excluded by the filtering process - 1988/89 to 1998/99 . . . . . . . 165

A.2 Summary of regression model coefficients and statistics . . . . . . . . . . 166

A.3 Summary of supplementary regression model results, excluding outliers . . 167

A.4 Summary of regression model variables using censored data . . . . . . . . . 171

A.5 Conditions for $T_{0}$ to be consistent with an NPE outcome assuming $\pi \sim U(0,1) 197$

A.6 Potential PBE outcomes and the specialist's second stage payoff . . . . . . 202 


\section{List of Figures}

2.1 Relative frequency of cost weighted discharges $\ldots \ldots \ldots$. . . . . . 30

2.2 Average cost weight of publicly-performed procedures over time $\ldots \ldots .32$

2.3 Average cost weight of privately-performed procedures over time . . . . . . 32

2.4 Number of publicly-performed procedures $\ldots \ldots \ldots \ldots$

2.5 Number of privately-performed procedures $\ldots \ldots \ldots$. . . . . . . . 34

$2.6 \quad$ Relationship between age and cost weight . . . . . . . . . . . . . 35

2.7 Residual plot of Model $1 \ldots \ldots \ldots$

2.8 Semi-parametric model of cost weight and time (Model 1a) . . . . . . . . . 40

2.9 Model of cost weight including a linear time trend (Model 4) . . . . . . . 44

2.10 Model of cost weight including a quadratic time trend (Model 5) . . . . . . 44

2.11 Residual plot of Model 4 . . . . . . . . . . . . . . . . 46

2.12 Residual plot of Model 5 . . . . . . . . . . . . . . . 46

3.1 Game tree for budget-bonus model $\ldots \ldots \ldots \ldots$. . . . . . . . 57

3.2 Cost function examples $\ldots \ldots \ldots \ldots$

4.1 Game tree for cheap talk model $\ldots \ldots \ldots \ldots$. . . . . . . . 87

4.2 Example of equilibrium outcomes with various $T_{0}$ for a range of $\theta \quad \ldots 103$ 
5.1 Game tree for three-actor model . . . . . . . . . . . . . . . . . . . . 116

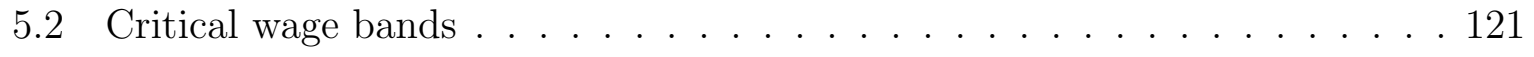

$5.3 \quad$ Outcomes in neologism-proof subgames . . . . . . . . . . . . . . . . . 125

5.4 Game tree for three-actor model with commitment step . . . . . . . . . . . 132

A.1 Relationship between cost weight and age, for inlier cases . . . . . . . . . . 169

A.2 Frequency of inlier cases by age . . . . . . . . . . . . . . . . . . 169

A.3 Relationship between cost weight and age, for outlier cases . . . . . . . . . 170

A.4 Frequency of outlier cases by age . . . . . . . . . . . . . . . . 170

A.5 Model of cost weight with a linear time trend (model 4-3), censored data . 172

A.6 Model of cost weight with a quadratic time trend (model 5-3), censored data 172

A.7 Model of cost weight with a linear time trend (model 4-6), censored data . 173

A.8 Model of cost weight with a quadratic time trend (model 5-6), censored data 173

A.9 Game tree for simple delegation model . . . . . . . . . . . . . . . . . . . . 177

A.10 Example of $F^{\prime}$ 's payoffs under delegation . . . . . . . . . . . . . . . . . . . 182

A.11 Game tree for first SBC model . . . . . . . . . . . . . . . . . . . . . . 185

A.12 Example of NPE outcomes for a range of $\gamma_{i}$. . . . . . . . . . . . . . . . . 195 


\section{Chapter 1}

\section{Introduction}

Every health system in the world appears to be beset with difficulties, which ought to be a warning to reformers that there are no easy solutions.

- Easton (1997)

New Zealand's public hospitals were reformed into Crown Health Enterprises (CHEs) in the mid-1990s. The reforms aimed to address the hospitals' persistent budget deficits and performance failures. The CHE reforms were part of a suite of reforms that aimed to liberalise the economy and commercialise parts of the public sector (Easton 1997).

The CHE reformers aimed to harness market forces through a corporate structure and to create stronger incentives for performance. CHE chief executive officers (CEOs) were given strong performance incentives aligned with the reformers' aims for the public hospital system. These incentives were expected to create a healthy tension between managers, as the health system funder's agents, and medical providers. The reformers expected the CEOs' incentives would flow down through the hospital hierarchy, improving performance throughout the organisation's hierarchy (Vaithianathan 1999b, 325).

Although the reforms created tension in the public hospital system, the reforms had unanticipated effects and were subject to major criticisms. Ultimately these criticisms contributed to the replacement of the CHEs. 
The central objective of this thesis is to analyse formally the underlying mechanics that could cause actual performance to differ markedly from that expected by the reforms' designers. We address the conceptual puzzles inherent in these criticisms, which may shed light on the CHE reforms and their failure to live up to expectations.

This thesis focuses on three criticisms of the operation of the CHEs about which the theoretical literature offers limited insight. The criticisms are that:

1. CHE Boards did not provide strong incentives as managers were paid bonuses despite failing to achieve performance targets.

2. Although managers were given strong incentives for cost control, these incentives impaired the relationship between managers and doctors, and resulted in poorer cost control.

3. The reforms gave medical specialists the opportunity and incentives to manipulate managers politically to the detriment of hospital efficiency.

We contribute to the theoretical literature by developing novel game theoretic models to explain stylised facts about the CHE reforms. Our models show how contractual incompleteness could disrupt the flow of beneficial incentives within a multi-layered organisational hierarchy. Market incentives can potentially be harnessed efficiently in such an environment. But there is a risk of over-sharpening incentives. We conclude that the CHE reforms could have over-incentivised managers and created too much tension, resulting in inefficient behaviour rather than solving the hospitals' perceived problems. Complex organisations that rely on the communication of soft information require systems that enhance trust and collaboration, rather than create unhealthy tension.

\subsection{Organisation of the thesis}

In this thesis, we formalise the CHE criticisms and assess them with reference to the concept of an organisational hierarchy. We consider an organisational hierarchy with 
four actors: a funder, an (hospital) administrator, a (medical) specialist and a (health) consumer. Each of the three theoretical modelling chapters focuses on the relationship between actors in this hierarchy.

We begin with an empirical motivation for the theoretical analyses in the subsequent chapters. Chapter 2 reports an empirical analysis of hospital usage and cost data over the reform period. We find that the CHE reforms were independently associated with an increase in hospitals' treatment costs.

Chapter 3 analyses how a funder could influence an administrator through budget targets and performance bonuses. In this chapter the administrator performs two roles. The first is as an allocator who is delegated authority to make rationing decisions. The second role is that of a specialist provider, who has private information about a patient's characteristics that affect which treatment is appropriate. A novel result of our model is that paying bonuses, despite apparent performance failures, can be a feature of efficient contracting. Chapter 4 separates the roles of an administrator and a provider, which were combined in Chapter 3, between two different actors. We consider how increasing the tension between an administrator and specialist might affect cost control. The model shows that sharper incentives for an administrator to control costs can impair communication with a specialist. A central and novel result of Chapter 4 is to show formally that sharper incentives to control costs, such as a tighter initial budget, can cause costs to increase.

Chapter 5 adds a consumer to the organisational hierarchy, and considers how conflicting incentives might erode a specialist's credibility with a consumer. We examine how a specialist's dual role as service provider and consumer advocate affects the specialist's credibility with the public. The model in this chapter is motivated by the results of Chapter 4, where some patients may be given inappropriate treatment as a result of the funder changing an administrator's incentives for cost control. Chapter 5 shows that the administrator may want the specialist to act as a patient advocate. A second result shows when an administrator may prefer to use managerial initiatives to sway the public and erode the specialist's credibility with the public, thus reducing valuable communication. 
Chapter 6 concludes by drawing together the insights from the empirical and theoretical analyses. It discusses how the findings provide a mutually reinforcing explanation of how reforms designed to improve a system could have failed to meet the reformers' expectations and generated criticism. The CHE reformers expected performance incentives to flow through a corporate structure to improve efficiency. This thesis argues that, rather than a cascade of beneficial incentives, stronger incentives for cost control can perversely create tension. Such tension can causes costs to rise, necessitating budget revisions and additional bonus payments, while permitting administrators to silence whistle-blowers in the system.

\subsection{Background literature}

The thesis draws on two streams of literature. One stream reflects on the health reforms in New Zealand. Section 1.2 provides an overview of the motivation and key features of the CHE reforms, the performance of the CHEs relative to the reformers' and others' expectations, and how, in some cases, performance worsened under the CHEs.

The second stream of literature formally models the effects of incentives on performance. In this stream, microeconomic theory is used to analyse agency failure in the presence of incomplete contracts. We present the modelling literature in two sets. The first set in section 1.2 provides background on agency theory and incomplete contracting, which gives a general underpinning to our models. The second set relates to specific modelling approaches and concepts, and sits in the relevant chapters.

\section{New Zealand's public hospitals and their reform in the mid-1990s}

Public hospitals have provided the majority of hospital services in New Zealand since the enactment of the Social Security Act 1938. In 1992/93 they accounted for approximately two-thirds of national health expenditure (excluding hospital deficit funding) and provided just over 90 percent of all hospital operations (Ministry of Health 2005). 
The CHE reforms in the early 1990s occurred in a broader context of substantial public sector reform. Many of New Zealand's public sector operations were retrenched during the 1980s and 1990s. The public sector reforms were motivated partly by contemporary fiscal pressures and "dissipating" support for the welfare state that was envisioned by the Government in 1938 (Boston, Dalziel \& St John 1999, 3).

Within the broader context of economic liberalisation and corporatisation, New Zealand's health sector reformers aimed to introduce market disciplines to a predominantly publicly controlled sector. This approach was inspired by the United Kingdom's internal market model. This approach maintained the predominance of public ownership and funding of hospitals, while aiming to introduce competitive disciplines via an internal market. This included the separation of funding, purchasing and provision roles, the latter being termed a purchaser-provider split. The broader health sector reforms, which include the CHE reforms, have been described in detail by Vaithianathan (1999b). 1

Prior to the 1993 health sector reforms, 14 Area Health Boards (AHBs) owned the public hospitals and controlled the 29 hospital boards that ran the hospitals. AHBs received money from government "to provide an unspecified range and quantity of services" (Hospital and Related Services Taskforce 1988, 23). Therefore, an AHB acted as both the purchaser and provider, determining what services to buy, and providing those services through its facilities. Prior to the health sector reforms, there were concerns that AHB purchasing decisions were biased by AHBs also being service providers. In addition, many hospitals ran persistent, substantial budget deficits. The aggregate AHB deficit climbed to $\$ 66.1$ million in 1992/93 (Deloitte, Touche \& Tohmatsu 1998).

In light of concerns about hospital efficiency, the Government targeted public hospitals for reform (Upton 1991). The Ministers of Finance and Health instituted a committee, the Hospital and Related Services Taskforce (HRST) ${ }^{2}$ This committee was charged with

\footnotetext{
${ }^{1}$ A market-orientated public health sector was a radical conceptual departure for New Zealand. While some researchers are sceptical about the extent of change to medical practice (Davies, Ashton \& Brown 2001), political considerations limited the pace and extent of change towards a market orientation. For example, the reformers designed quasi-market institutions but did not to privatise major public hospitals.

${ }^{2}$ The thesis refers to the Ministers of Finance and Health, and The Treasury and Ministry of Health,
} 
reviewing the New Zealand public secondary care (hospital) system.

One conclusion of the Hospital and Related Services Taskforce (1988) was that the interests of hospital administrators were closely aligned with those of clinical specialists, but were not well aligned with the health service funder's interests or responsive to the public's priorities (Hospital and Related Services Taskforce 1988, 6-12). The report critically noted that"many [area health] boards seem to think expenditure on accounting and information services is a waste of money and would rather spend it on clinical services staff" (Hospital and Related Services Taskforce 1988, 21).

The Taskforce also concluded that "the government has largely delegated control of its central concern for equity to health professionals who thus, by default, have assumed progressively more powers over areas for which they have received no specific training or brief" (Hospital and Related Services Taskforce 1988, 5). It argued that the environment sponsored a lack of cost consciousness where "the use of the bulk of the hospital's resources is determined by the decisions of individual doctors and nurses who have clinical but not resource responsibility", resulting in poor resource allocation decisions (Hospital and Related Services Taskforce 1988, 21).

Arthur Anderson and Co was commissioned by the HRST to report on the relative efficiency of the hospital sector. It estimated that between $\$ 450$ million and $\$ 600$ million was wasted in 1986/87 due to poor hospital management (Arthur Anderson and Co 1987a). The estimates implied wastage of between approximately one-quarter and one-third of total public hospital operating expenditure at that time. $3^{3}$ The lower estimate of the wastage was contextualised as being "equivalent to the 1986/87 government grant for the Auckland Hospital Board for operating expenses", while the upper figure was "almost as large as the combined operating grant to the Auckland and Canterbury Boards, the two largest boards in the country" (Hospital and Related Services Taskforce 1988, 13).

collectively as the reformers, or the funder, of the public health system.

${ }^{3}$ Arthur Anderson and Co's (1987a) estimates were based on an annual operating expenditure of $\$ 1.85$ billion, and noted that even the upper estimate was regarded as "conservative". Others later questioned the robustness of these figures (Easton 1997). However, the estimates indicate the reformers believed that substantial inefficiency existed in the AHB hospital system. 
The Taskforce's report, "Unshackling the hospitals", recommended splitting purchasing responsibilities from provision, as part of establishing an internal market. This split was consistent with Arthur Anderson and Co's (1987a, I-2) conclusion that "hospital and board management structures need to be sharpened", and the funder and provider functions of the health board be separated to "set up a benign but healthy tension leading to greater result from expended funds". The purchaser-provider split and corporatisation of New Zealand's public hospitals was expected to sharpen organisational incentives for cost efficiency (Hospital and Related Services Taskforce 1988, Upton 1991, Easton 1997).

The reformers and their advisors suspected hospital managers had soft incentives for operational efficiency due to: a team-based approach to management involving a "triumvirate" of an Administrative Officer, a Medical Superintendent and a Principal Nurse; a non-profit ethos; and a lack of management information (Hospital and Related Services Taskforce 1988). A Crown Company Monitoring Advisory Unit (CCMAU) ${ }^{4}$ observer expressed of one hospital that "there was insufficient tension between management and clinical staff" (Health and Disability Commissioner 1998, 164).

Arthur Anderson and Co (1987a) found managerial information systems were weak under the public AHB model. Corporatisation was expected to improve the availability and quality of managerial information available. The drive to use private sector management techniques to improve information availability was accompanied by oversight from the Crown Company Monitoring Advisory Unit as part of an effort to make "rationing more explicit” (Crown Company Monitoring Advisory Unit 1996, 6).

Cabinet directed that hospitals should be reorganised into corporations in the early 1990s (Upton 1991). The reformers expected better information, coupled with strong managerial incentives, to facilitate prioritisation, deliver efficiency gains, and reduce hospital deficits.

Hospitals became financially-independent public companies called Crown Health Enter-

\footnotetext{
${ }^{4} \mathrm{CCMAU}$ was a government agency that was charged with monitoring the performance of public hospitals as state-owned companies and providing advice to the shareholding Ministers of Finance and of Health about "maximis[ing] the value from owning these companies". Downloaded 25 August 2004 from http://www.ccmau.govt.nz/aboutUs/whatwedo.asp.
} 
prises, governed by a Board of Directors, and managed by CEOs. CHE boards and managers were driven by the dual, but sometimes conflicting, statutory objectives of social responsibility and operating as a successful business (Ashton 1999, Upton 1991, 148). CEO's contracts included service and budget targets, with bonuses tied to improving operational efficiency. The incentives created for upper management were supposed to cascade to lower levels of the hierarchy to reinforce the efficiency objective.

The reforms gave CHEs "enhanced powers to negotiate individual employment contracts with medical staff" (Kisely 1997, 2). CHE managers' efforts to pass on their incentives led to a warning from the Director of the Association of Salaried Medical Specialists in New Zealand that "management is promoting alternative individual contracts which significantly enhance managerial power over what doctors do, when they do it, and what they are paid for it" (Williams 1996, 578). Despite contractual obligations, doctors were bound by a statutory set of medical ethics focused on patient wellbeing (Medical Council of New Zealand 1994).

The reforms sharpened managers' incentives for cost control, but by 1996 "the health reforms [had] yet to yield the original expectations... the pace of performance seems, if anything, to have weakened since the advent of the reforms" (Crown Company Monitoring Advisory Unit 1996). Instead of universally improving operational efficiency, corporatisation saw accelerating deficits in some hospitals. The government injected extra funds through specific budget appropriations for CHE deficits. "From 1993/94 to 1997/98 CHE deficit financing averaged $\$ 202$ million per annum ... The deficits decreased to $\$ 39.6$ million in 1998/99 and $\$ 6.4$ million in 1999/2000 because of increased funding through Vote Health" (Ministry of Health 2001a, 4). These injections were over $5 \%$ of the Ministry of Health's funding to health purchasers, and reached 11\% in 1996/97 (Ministry of Health 2001a, Vaithianathan 1999b). The Ministry of Health mainly attributed the deficits over this period to "underpricing of services and provision of more services than CHEs/HSPs were contracted to provide" (Ministry of Health 2001a,4).$^{5}$

\footnotetext{
${ }^{5}$ Public sector health expenditure rose more quickly in the five years over the CHE reforms (at an annual average rate of $4.4 \%$ ) than in the preceding five years (1.3\%), in real terms (Ministry of Health
} 
The reforms were strongly criticised by members of parliament, alienated some medical staff and contributed to growing public disaffection (Health and Disability Commissioner 1998, Ministry of Health 1998, Donelan, Blendon, Schoen, Davis \& Binns 1999) $]^{6}$ In its 1996 briefing to the incoming minister, CCMAU acknowledged that the reforms had not "fully met its expectations" or "achieved their full potential" (Crown Company Monitoring Advisory Unit 1996, 7-8).

The Medical Council of New Zealand (MCNZ) is responsible for regulating medical practice and guiding doctors in areas such as standards and conduct.7 The second ethical principle of the Medical Council of New Zealand's (1994) guidelines for doctors in a resource constrained environment stated, it is "not acceptable for doctors to allow their commercial interests, or those of an employer, to affect their clinical decisions in meeting the health needs of individual patients..." 8 Vaithianathan $(1999 a, 171)$ points to one case, where a team of hospital doctors refused to fulfil a contract negotiated by a CHE manager to treat patients from another hospital. In the doctors' belief, such treatment amounted to unethical queue jumping. This suggests that CEOs may not have secured sufficient authority to implement some cost control innovations.

New Zealand's health service funder may also have had greater difficulty designing incentives to encourage cost control by a doctor than an administrator. Hospital managers were not governed by a professional code that included a principle of patient care, unlike doctors. 9 Near the end of the reforms, however, a number of CHEs began to appoint clinical

$2001 a, 12)$. This trend was reflected in public expenditure per capita, in spite of an increasing population. Public sector health expenditure also grew more quickly than private expenditure (4.0\%) over this period, in contrast to the pre-reform period when private expenditure grew more quickly. The public share of expenditure rose from $76.6 \%$ of the total prior to the reforms in $1992 / 93$ to $77.0 \%$ in $1997 / 98$.

${ }^{6}$ A public opinion survey by the New Zealand Ministry of Health in 1998 and by international researchers in 1999 indicated increasing dissatisfaction with New Zealand's public health care system. The percentage of New Zealanders who thought that the health care system needed to be completely reformed rose from 18\% to $32 \%$ between these surveys (Ministry of Health 1998, Donelan et al. 1999).

${ }^{7}$ The role of the MCNZ is paraphrased from the Council's website. Downloaded 17 February 2006 from http://www.mcnz.org.nz/

${ }^{8}$ The 1994 guideline was updated and more strongly named an "ethical guideline" in 1999 (Medical Council of New Zealand updated October 1999). Although the later document was published after the CHE reforms, the four "ethical principles" that begin both documents remained the same.

${ }^{9}$ Although the MCNZ's guidelines, Responsibilities of Doctors in Management and Governance, were 
directors to enhance clinical input to management decision-making (Mold 1996, Crown Company Monitoring Advisory Unit 1996, Health and Disability Commissioner 1998).

The Crown Company Monitoring Advisory Unit's (1996) Briefing to the Incoming Minister recognised the public criticisms of the CHE reforms, and some of the perceived failings underpinning these criticisms. It observed that 21 out of 23 CHEs were in deficit in 1996, and argued that "deficit support has the perverse effect of rewarding poorer performance with more money" (Crown Company Monitoring Advisory Unit 1996, 8). CCMAU also noted that the public's concerns about the reforms "may or may not accord with reality, but... the public's concerns have to be acknowledged in the development of policy since a measure of public acceptance is necessary for policy stability over time" (Crown Company Monitoring Advisory Unit 1996, 6).

Ultimately, flagging operational efficiency, growing deficits, medical protest and public disaffection with the reforms saw the Government resile from the corporatisation of public hospitals. The Government replaced CHEs with non-profit Hospital and Health Services (HHSs) in mid-1998.

\section{Agency theory, incomplete contracts and authority}

Over the next four chapters we consider how corporatisation and strong managerial incentives could fail to deliver on the reformers' expectations. As a backdrop to this analysis, we briefly review some of the foundational literature on principal-agent relationships, incomplete contracts, authority and organisational hierarchies.

Agency theory provides insight into how relationships function and how mechanisms, such as contracts, can be used by a principal to provide an agent with appropriate incentives. The agency theory literature "recognizes conflicts of interest between different economic actors" within organisations, and "formaliz[es] these conflicts through the inclusion of ob-

published after the CHE reforms, they reflected on incidents that occurred during the reform period. This guideline states "[medical managers'] first consideration must continue to be the interests and safety of patients regardless of their managerial responsibilities" (Medical Council of New Zealand 2000, 1). 
servability problems and asymmetries of information" (Hart 1989, 1758-59). Information asymmetries and observation or monitoring costs mean contractual incompleteness plays a central role in agency theory (Hart \& Moore 1985, Tirole 1999). A contract may be incomplete because it is not feasible or desirable to specify precisely each agent's obligations in every contingency (Williamson 1985, Bernheim \& Whinston 1998). Contractual incompleteness means that in some circumstances it is more efficient to organise economic activity within a firm than through a market (Grossman \& Hart 1986).

Alchian \& Demsetz (1972) build on the concept of authority within a firm developed by Simon (1951). This body of work emphasises that organisations, such as firms, give managers decision making power and incentivises them as a residual claimant.10 As Tirole (1999) puts it "a decision right or authority granted to a party is the right for the party to pick a decision in an allowed set of decisions". Organisational theorists provide the useful framework an organisational hierarchy, and consider how features of this hierarchy affect the ability of a manager to exercise delegated authority (Aghion \& Tirole 1997, Harris 1977).

We use the framework and concepts of authority and delegation to consider how the CHE reforms may have altered authority within a hospital hierarchy. Aghion \& Tirole (1997) introduce the ideas of formal and real authority to understand decision-making in hierarchical organisations. Formal authority refers to "the right to decide". Real authority is "effective control over decisions", that is, having sufficient information to make an informed decision (Aghion \& Tirole 1997, 1-2). Formal authority need not confer real authority, and Aghion \& Tirole (1997) emphasise the important role of information in determining the locus of real authority. That paper considers how formal authority can affect the information structure within an organisation, and models the communication of "hard" (verifiable) information. The authors conclude that a principal may do better by accepting a subordinate's informed, but biased, recommendation rather than making an uninformed choice themselves.

\footnotetext{
${ }^{10} \mathrm{~A}$ residual claimant is an agent who is rewarded with (a share of) the surplus of income over costs.
} 
In the context of the health sector, Harris $(1977,470)$ emphasises that hospital doctors have a substantial degree of independence from administrators due the "unstandardized" nature of medical services, combined with ethical and legal factors. A manager may limit this autonomy due to a doctor's reliance on the hospital's "ancillary services", such as a pharmacy. In addition to the factors outlined by Harris (1977), Vaithianathan (1999a, 173-175) notes that a doctor's real authority depends on the information he controls relative to a manager, and the doctor's alternative opportunities if he is excluded from using a particular hospital's assets.

Agency theory is closely connected to the area of information economics. Holmström (1982), for example, considers how competition within a team can create incentives that can be used to extract information. In Arrow's (1963) early application of information economics to the health sector, he explores how information asymmetries pose difficulties for the efficient functioning of health markets and institutions within them. Vaithianathan (1999a) adds to this literature by modelling mechanisms to overcome information problems faced by health insurers and in primary care reimbursement schemes; and, analysing the internal market created by New Zealand's health sector reforms.

\section{Discussion}

This literature forms a backdrop to our analysis of incentives within a stylised CHE organisational hierarchy. In Chapter 2, we complement this literature review with an empirical motivation for our theoretical models. We show that the CHE reforms were associated with an increase in treatment costs in public hospitals, in spite of the incentives for improved efficiency outlined above.

In later chapters, we build on Chapters 1 and 2 by arguing that incomplete contracts in a multi-layered hierarchy could cause unintentional negative interactions between the agents. Tension and perverse incentives could result in the sort of criticisms made of the CHE reforms. We show how a set of reforms designed to improve a failing system could fail to achieve even their primary objective of solving the health system's deficit problem. 


\section{Chapter 2}

\section{Empirical investigation: hospital}

\section{admission and cost data, 1988-1995}

\section{$2.1 \quad$ Introduction}

This chapter aims to establish the plausibility of the claim that CHE costs did not fall as expect. This claim underpins the criticisms that this thesis examines formally in the following three chapters. That is, despite expectations of improved efficiency, costs fell less quickly and efficiency gains were less than expected. In Chapter 4 we show formally how sharper managerial incentives for cost control can even have the perverse result of increasing costs.

This chapter answers the following empirical research question.

Were the CHE reforms independently associated with an increase in the average reported cost of medical and surgical procedures in public hospitals?

We analyse hospital use and treatment cost data from July 1988 to June 1995 to identify whether the reforms were associated with an increase in average hospital treatment costs. 1

\footnotetext{
${ }^{1}$ Although the CHEs were restructured in 1998, the shorter period to 30 June 1995 avoids confounding our analysis due to data coding changes and funding injections that occurred from 1 July 1995.
} 
We believe that this is the first analysis to use the National Minimum Data Set (NMDS) to estimate econometrically the effect of the CHE reforms on reported inpatient treatment costs. We find that the CHE reforms were independently associated with an increase in hospitals' average treatment costs, which contrasts with the objective of the reforms to control costs and improve efficiency.

The chapter is organised as follows: section 2.2 provides a brief contemporary background to the reforms to give a political and economic context for the empirical analysis. Section 2.3 describes the data and core analytical variables we use for this analysis. Section 2.4 details the method and assumptions, and section 2.5 reports the results. The final section discusses what the econometric results reveal about the impacts of the reforms.

\subsection{Background}

The CHE reforms introduced corporate structures and sharpened hospital managers' incentives in order to bring hospitals' deficits under control, to improve the prioritisation of care and improve efficiency. Contemporary statements by some CEOs suggested that they felt they had a stronger focus on cost control than the hospital management teams under the preceding AHB system (Coast Health Care Limited 1994, Healthlink South 1996, Guyan 1998).

While the reformers successfully created cost-control incentives for managers, these incentives conflicted with the incentives of a significant proportion of medical staff, due to perceived clashes between a profit motive and a medical professional code that included a principle of patient care (Easton 1997, Health and Disability Commissioner 1998). In turn, this conflict impaired the relationship between managers and clinical staff in some hospitals (Health and Disability Commissioner 1998).

Chapter 1 and the contemporary evidence above furnish three stylised facts about the CHE reforms. These stylised facts form a backdrop to the empirical analysis in this chapter and theoretical analysis in the following chapters. The first is that the CHE 
reforms successfully sharpened managers' incentives to control costs. Second, the CHE managers' incentives were not well aligned with a significant proportion of clinicians' incentives. A corollary of the first two statements is that the CHE reforms caused, or increased, a misalignment between the incentives of hospital managers and clinicians than existed under the AHB public hospital system. The third stylised fact is that increased tension between managers and doctors worsened relationships between these two groups.

\subsection{Hospital admission and treatment cost data}

The New Zealand Health Information Service (NZHIS) extracted data from its NMDS for this research. The NMDS is an administrative data set containing discharge record data for publicly and privately-performed hospital procedures.$^{2}$ Most procedures relate to medical and surgical care $3^{3}$

The NZHIS uses the data provided by hospitals to construct summary treatment and cost-related variables, such as a Diagnostic Related Group (DRG) code $4^{4}$

NMDS data on publicly-funded hospital events are available from 1988. Data are recorded for July years, corresponding to a financial year from 1 July to 30 June. The NZHIS also has discharge data up to 1995 for privately-funded procedures. The latter data are not relevant as the private hospital sector was not subject to the CHE reforms and are unsuitable for comparison to public hospital data (see footnote 2). Below, "private procedures" refers to publicly-funded procedures performed in private hospitals.

Each discharge record has a patient identifier called a National Health Index (NHI) num-

\footnotetext{
${ }^{2}$ The NMDS also contains records on privately-funded procedures that were performed in public or private hospitals. NZHIS advised against comparing private data with public data as the data are collected differently and the private records have more missing information. The missing information mostly relates to diagnoses, especially co-morbidities, and complications. The absence of this information results in the discharge being allocated a low costweight and "an overall perceived lack of complexity irrespective of what actually happened to the patient" (personal communication with NZHIS, 21 June 2005).

${ }^{3}$ The NMDS also covers procedures related to mental health, geriatric and maternity services.

${ }^{4}$ Diagnostic Related Group (DRG) codes categorise discharges based on the diagnosis made and procedure(s) undertaken (Ministry of Health 2001b).
} 
ber.5 NZHIS supplied records with anonymised NHIs for this research. The anonymised

NHIs are consistent across across all the years of data for a given NHI number.

\section{Cost weights}

We use cost weights as our main dependent variable. A cost weight is an index of the average cost of treating a particular type of medical or surgical procedure ${ }^{6}$ Cost weights are useful for this research as they reflect the real resources used to treat a patient.

A cost weight depends on both the patient's health status and the interaction between managers and clinicians, which result in the authorised treatment. That is, the reported cost weight reflects what the patient needed, as evaluated by a medical expert, and what was authorised in accordance with hospital policy set by management.

Cost weights are calculated using a case-mix methodology. The case-mix reflects the complexity and costs of cases that a hospital treated (Ministry of Health 1999, 233). It was used by New Zealand health providers, and purchasers, to monitor hospital throughput.7 The NZHIS assigned a cost weight to each inpatient discharge record and can construct cost weights using individual patient discharge records from 1988.

\footnotetext{
${ }^{5}$ Individual patients are assigned a unique NHI number on the person's first contact with a public hospital. Subsequent admissions by the same individual should be recorded using their unique NHI number. The NZHIS transformed individual NHI numbers into an anonymous patient identifier for confidentiality reasons as a requirement of providing the data for this research.

Approximately 18.0 percent of patients had more than one admission in the period covered by the data set. Around half of these admissions were within a single year. Only 9.5 percent of patients had admissions in more than one financial year, and the majority of these (5.9 percent of patients) were in no more than two different years.

It is possible that a single person is assigned more than one NHI number in error. In 2003, the Ministry of Health found 125,000 duplicate NHI numbers, which was approximately 2.5 percent of the active NHI numbers. However, non-unique NHI numbers are primarily an issue for tracking patients across time, so it is not a material issue given the focus of this research.

${ }^{6}$ The funding a hospital would attract for a given case was calculated as the reported cost weight, which are also referred to as case-mix weight, multiplied by a base case-mix price set by the government's health service purchaser. The NZHIS standard procedure normalises the base cost weight to 1.0 each financial year. A case with a cost weight of 1.0 would attract, for example, $\$ 2,433.62$ of funding in $1998 / 99$, which reflected the average funding per inpatient in that year.

${ }^{7}$ Between 1993 and 1999 the government had several health service purchasing agents, variously Regional Health Authorities, the Transitional Health Authority and the Health Funding Authority.
} 
The cost weight applied to a particular record depends on several factors. These factors include the reported diagnoses and procedures a person received, which affect the DRG code that a case is assigned, the person's length of stay and whether the person had complications or required mechanical ventilation (de Boer 2000, Rains, Hogan \& de Boer 2001). The NZHIS generates cost weights according to a well defined methodology called the Weighted Inlier Equivalent Separations (WIES) methodology (de Boer 2000, Rains et al. 2001). This research uses Version 5 with Amendments for New Zealand (WIES5A). Appendix A.1.1 outlines this methodology and details its application in this research.

We calculated a consistent index for every admission record across the entire analytical period using: the WIES5A methodology; a single version of the DRG classification system; and, a common cost weight base for all years of data, rather than re-basing the base cost weight to 1.0 for each financial year. By applying a single version of this methodology to the admission data, we avoid spurious results from methodological changes over the analytical time period, periodic re-weighting of the relative cost of specific treatment categories and general hospital service inflation.

Applying the cost weight methodology in this way has a number of advantages. It:

- provides a consistent measure of reported inpatient treatment costs before and after the reforms, calculated using the same cost-mix methodology for all years of data

- excludes costs such as overheads and reform implementation costs that would cloud the reforms' effects on operational efficiency within a hospital

- represents a real price index

- directly reflects reported diagnostic and treatment information, rather than the funding a case attracted, which also depends on the case-mix base price and thus may be confounded by the periodic changes to the case-mix price (see footnote 6).

By constructing the cost weight variable specifically for this research, we can interpret changes in the average cost weight over time as a change in the (average) real resources 
used to treat a patient. Other demographic data tied to the NHI let us control for a range of other influences that affect the cost weight, so that we can isolate the effect of the reforms on the average cost weight.

\subsubsection{Data cleaning and filtering}

This research compares data collected across time and from different providers. Meaningful comparisons require data that are prepared and reported in a consistent way throughout the analytical timeframe. Therefore, the data were filtered to improve the coding and reporting consistency. Below we summarise this process.

We exclude publicly-funded, privately-performed procedures from the econometric analysis. In addition to concerns about data quality for privately-performed procedures, it is unclear that the CHE reforms would have had the same effects in private hospitals as in public hospitals. Private hospitals were for-profit organisations or non-profit hospitals run by private interests. We consider the privately-performed procedures in the univariate and bivariate analysis only $\left.\right|^{8}$

\section{Timeframe}

The main analysis uses data from 1 July 1988 to 30 June 1995. Data after 1995 are subject to changes in coding by hospital staff due to changes to the WIES methodology, and ad hoc funding injections to address patient waiting times in 1995 and 1997.99 These changes affected how the data were reported, while the funding injections affected the health status and treatment cost of the average patient. The coding changes are expected to result in higher reported costs after 1995, while the funding injections could potentially lower the average cost weight by improving access for lower priority patients and shortening waiting

\footnotetext{
${ }^{8}$ This analysis shows the importance of allowing for the impact of the funding injections in 1995 and 1997, due to changes induced in the mix of patients and proportion of patients treated in public hospitals as private hospital provision increased.

${ }^{9}$ Appendix A.1.1 discusses the coding changes and funding injections in more detail.
} 
times (Fielden, Cumming, Horne, Devane, Slack \& Gallagher 2005). These changes could disguise the effect of the CHE reforms on reported patient costs.

Focusing on the period between 1 July 1988 and 30 June 1995 gives a total of 3,862,495 records $(3,736,814$ records, after filtering the data and excluding private procedures) 10

\section{NZHIS filtering process}

We concentrate on publicly-funded procedures because the data are reported most consistently. We use the NZHIS standard NMDS filters (Ministry of Health 2000, Ministry of Health 2001b)111 Appendix A.1.2 discusses the main types of patients excluded from this analysis, and Table A.1 in this appendix tabulates the number of records affected by exclusion type.

\section{Summary of exclusions}

Table 2.1 on page 20 summarises the data between 1988 to 1999 that were excluded from the econometric analysis. The first three rows are based on the NZHIS' filters. The final row shows the privately-performed procedures that are excluded, and the third column shows the data from 1 July 1995 that are excluded from the main analyses ${ }^{12} 13$

We exclude 249,013 records (3.76 percent) from the original data set of 6,619,761 records. This gives an analytical data set with $6,370,748$ observations (96.24\% of the original

\footnotetext{
${ }^{10} \mathrm{We}$ conducted supplementary econometric analyses using an uncensored data set ranging from 1 July 1988 to 30 June 1999. The models in the supplementary analysis had similar sets of variables to the main analyses, with the inclusion of dummy and interaction terms for the coding changes and funding injections after 1 July 1995. These analyses did not generally show any qualitatively different results from the core models for the period up to 30 June 1995. The results of these supplementary analyses are not reported here.

${ }^{11}$ Appendix 2 of Ministry of Health (2001b)) discusses limitations in the NMDS data and the filtering rules it applies; some of these filters are not relevant to this research.

${ }^{12} \mathrm{~A}$ case may meet more than one exclusion criterion, so the total number of excluded cases does not equal the sum of the categories shown in table 2.1 .

${ }^{13}$ Cases assigned zero cost weights represented a small proportion of the total data set, with some difference in the propensity for public and privately-performed procedures. Just under 0.79 percent of the 6,544,461 publicly-performed procedures had zero cost weights, while the equivalent proportion was 0.58 percent of the 75,300 privately-performed procedures.
} 
data set) between 1988 to 1999. The majority of the excluded cases were non-treated admissions or publicly-funded patients treated in private facilities. The main error DRG records were due to an unacceptable primary diagnosis (AN-DRG 956) 14

\begin{tabular}{|c|c|c|c|c|c|}
\hline Reason for exclusion & $\begin{array}{l}\text { July } 1988 \\
\text { - June } 1993\end{array}$ & $\begin{array}{l}\text { Number of } \\
\text { July } 1993 \\
\text { - June } 1995\end{array}$ & $\begin{array}{l}\text { excluded ca } \\
\text { July } 1995 \\
\text { - June } 1999\end{array}$ & Total & $(\%)$ \\
\hline Zero cost weight & 35,776 & 14,681 & 1,626 & 52,083 & $0.79 \%$ \\
\hline Non-treated admission & 52,652 & 18,326 & 26,213 & 97,191 & $1.47 \%$ \\
\hline Error DRG & 35,776 & 14,680 & 1,630 & 52,086 & $0.79 \%$ \\
\hline Non-case-mix procedure & 1,699 & 3,239 & 20,426 & 25,364 & $0.38 \%$ \\
\hline Private procedure & 13,659 & 5,717 & 55,924 & 75,300 & $1.14 \%$ \\
\hline Total & 139,562 & 56,643 & 105,819 & 249,013 & $3.76 \%$ \\
\hline
\end{tabular}

Table 2.1: Data excluded using NZHIS filters and due to a private procedure

\subsection{Method}

We examine the impact of the reforms by examining individual discharge records. We begin by presenting descriptive statistics and bivariate patterns. These provide an overview of data characteristics and the simple relationships between the analytical variables. This stage does not control for differences in variables that are likely to influence treatment costs, such as age. Such influences are controlled for in the econometric analysis stage. Changes in the age distribution over the analytical timeframe, for example, would affect the average treatment profile and the average cost weight.

We then use econometric methods to control for the effects of several variables on cost weight, in order to isolate the relationship between the reforms and reported treatment costs. The primary econometric method used is multivariate ordinary least squares (OLS) regression. We regress cost weights on a dummy variable for the reforms, named period,

\footnotetext{
${ }^{14}$ The distribution of cases that were assigned error DRGs is: AN-DRG $951 \mathrm{n}=8,319$; AN-DRG 952 $\mathrm{n}=100 ;$ AN-DRG $955 \mathrm{n}=1,132$; and, AN-DRG $956 \mathrm{n}=42,535$.
} 
and control variables including age and a background time trend.

The period variable is a binary variable, and is used as part of the process to isolate the potential effects of the reforms from other changes over the reform timeframe. The period variable indicates whether a discharge occurred before or after the reforms, that is, 1 July 1993. It takes a value of 0 or 1 , respectively.

\section{Potential confounding variables}

A range of factors changed over the reform period, and these coincidental changes could obscure the effects of the reforms.

Patient age should be controlled for because as it affects the demand for, and cost of, treatment. Changes in the age distribution during the reforms would change the average cost weight, ceteris paribus. Health care needs tend to be high at birth, followed by a period of lower need, then rise at older ages as increasingly costly and complicated care is needed. We use age and $a g e^{2}$ variables in the regression model to isolate this hypothesised non-linear relationship with the cost-weight variable.

A background time trend in treatment costs could also obscure the impact of the CHE reforms. That is, unobserved time-related factors may cause cost weights to change. For example, the cost weight may fall over time due to improvements in medical technology that allow the substitution of cheaper, or more effective treatments. This could change the composition or average health of patients entering the system.

Two approaches were used to investigate the presence of a time trend and to isolate its impacts from the effects of the reforms. The first approach is an exploratory, semiparametric method that aims to control for an unobserved time-related effect without imposing a particular parametric model form on the relationship between cost weight and time. The approach uses a binary variable for each month, where there are $T$ months and the model uses $T-1$ dummy variables. 
The second approach assumes a parametric time trend ${ }^{15}$ This approach uses a single multinomial variable whose value equals the month of discharge. Based on the period 1 July 1988 to 30 June 1995, this variable has a range of 1 (July 1988) to 84 (June 1995). ${ }^{16}$

Hospital coding practices may also change over time as hospital managers respond to the influence of cost weight on hospital revenue and as system improvements allow more accurate and comprehensive recording. Steinwald \& Dummit (1989), for example, examine case-mix change in Medicare's prospective payment system. They distinguish between "real case-mix change" and "DRG creep". The latter refers to "changes in hospital recordkeeping practices to increase case-mix indexes and reimbursement", and is also known as coding creep.

In New Zealand, however, cost weights only had an indirect influence on funding as they were used as one of many inputs to contract negotiations and to update the general funding formula. They were not a direct funding mechanism to fund medical/surgical events during the study period for this analysis. In addition, prior to July 1995, there was limited scope under the original New Zealand coding rules for coding creep with records allowing just 4 diagnosis codes and 3 procedure codes. In July 1995, Australian coding rules were introduced, allowing mapping to Australian DRGs. This increased the number of diagnoses (from 4) and procedures (from 3) to 25 each.

Complication codes are an element of the discharge record. Their use can indicate that a case is more complex, more costly, and would therefore attract more revenue ${ }^{17}$ An examination of the proportion of cases with no complication code in 1992 and 1994 showed no statistically significant increase. This suggests that immediately around the reforms, which is the focus of this empirical analysis, there is unlikely to have been substantive coding creep. This is consistent with the limited scope for such behaviour under the New

\footnotetext{
${ }^{15}$ We examined linear, quadratic and cubic time trends. We present the results for the linear and quadratic forms. We do not present the results for the cubic form as they were similar to those using the quadratic form and less intuitive.

${ }^{16}$ Supplementary analyses using data beyond 1 July 1995 included dummy variables and interaction terms to isolate any structural break effects due to the funding injections in this period.

${ }^{17}$ Records can be coded on a four point scale, with zero indicating no complications.
} 
Zealand DRG coding system prior to July 1995, and the indirect effect of cost weights on hospital revenues.

\section{Issues with using cost weights to estimate the impact of the reforms}

Using cost weights to estimate how reported treatment costs responded to the reforms poses two issues.

The first issue is that meaningful cost weights should take a positive value, but it is possible to predict a non-positive cost weight using the OLS method. We investigated both linear and log-linear model forms ${ }^{18}$ The estimated linear models all predicted positive cost weight values for individual discharge records. Therefore, there was no issue in practice with interpreting the model results for this data set.

We report the results of a log-linear form for two core models to check on the robustness of using the linear form of the cost weight variable.19 The anti-log of the estimated coefficients from these models produced results that were qualitatively and quantitatively similar to the simpler linear models examined. This suggests that the analysis is robust to the linear form of the cost weight variable.

A second issue relates to the timing of potential impacts from the reforms. CHEs were legally established on 1 July 1993. Therefore any impacts of the reforms should occur after this date. The simple relationship between cost weight and time, shown in Figure 2.3 on page 32 , suggest that the reforms were associated with a change in the average cost of medical procedures. The main analysis in this chapter assumes that the reforms had immediate impact. This is captured by using a before/after dummy variable calibrated to 1 July 1993, when CHEs were legally established.

\footnotetext{
${ }^{18}$ Regressing on the natural log of the cost weight avoids the issue of non-positive predictions.

${ }^{19}$ The log-linear form has two drawbacks. First, this form implies that cost weight is proportionally related to the independent variables, which is arguably a stronger assumption about the relationship than a simple linear relationship as it places importance on the initial (average) level of the cost weight variable. Second, the estimated coefficients of this technique are more complicated to interpret, as they reflect relative rather than absolute changes.
} 
It is possible that the reforms took some time to take effect. For example, it may have taken some time for CHE managers to introduce changes and for these to flow through the layers of hospital hierarchy and thus affect costs. Such transitional timing would cloud the effect of the reforms and make it more difficult to detect a statistically significant impact. However, a supplementary analysis censoring a period immediately following the reforms did not find this was a significant issue ${ }^{20}$

\section{Econometric models}

This section specifies the econometric models used to analyse the data described above. We estimated nine models, which progress from identifying simple relationships to including an appropriate set of variables to isolate the effects of the reforms.

Table 2.2 on page 25 defines the variables used in the analysis. We use various interaction terms in some models, which are the product of the listed variables; Table 2.2 does not list the interaction terms separately ${ }^{21}$ For example, period $\times$ month equals 0 prior to the reforms when the period variable is 0 , and takes the value of month after the reforms. This interaction term allows us to detect the effect of the reforms on a linear time trend.

\footnotetext{
${ }^{20}$ This supplementary analysis estimated selected models using a data set that censored a proximate period following the reforms of three or six months. Section 2.5 .2 briefly discusses the results of this analysis and Appendix A.1.5 reports the results.

${ }^{21}$ The Break $k_{1}$ and Break $_{2}$ variables are used to control for funding and coding changes after 1 July 1995 in a supplementary analysis. These variables are not used in the main analyses, which concentrate on the period up to 30 June 1995 as discussed in section 2.3.1.
} 


\begin{tabular}{|c|c|c|}
\hline Variable & Туре & Definition \\
\hline$C W$ & Continuous & $\begin{array}{l}\text { Cost weight is a non-zero index of average treatment } \\
\text { cost assigned to a discharge record. }\end{array}$ \\
\hline Period & Binary & $\begin{array}{l}\text { An indicator of whether a discharge was before the } \\
\text { reforms }(=0) \text { or on/after } 1 \text { July } 1993(=1) \text {. }\end{array}$ \\
\hline Age & Discrete & Age in years as at the day of discharge. \\
\hline$A g e^{2}$ & Discrete & Based on Age; used to detect non-linear effects of age. \\
\hline Month & Discrete & $\begin{array}{l}\text { Month as at the date of discharge, ranging from } 1 \\
\text { (July 1988) to } 132 \text { (June 1998). }\end{array}$ \\
\hline Month $h^{2}$ & Discrete & $\begin{array}{l}\text { Based on Month; used to detect a non-linear } \\
\text { background cost trend. }\end{array}$ \\
\hline Break $_{1}$ & Binary & $\begin{array}{l}\text { An indicator of whether a discharge was before coding } \\
\text { and funding changes }(=0) \text { or on/after } 1 \text { July } 1995(=1) \text {. }\end{array}$ \\
\hline Break $_{2}$ & Binary & $\begin{array}{l}\text { An indicator of whether a discharge was before a } \\
\text { funding change }(=0) \text { or on/after } 1 \text { July } 1997(=1) \text {. }\end{array}$ \\
\hline
\end{tabular}

Table 2.2: Variable definitions

Table 2.3 outlines the variables included in the main models. All models were estimated with an intercept term. Table 2.8 on page 36 in section 2.5 .2 presents the regression results for the models outlined in Table 2.3 .

\begin{tabular}{|c|c|c|c|c|c|c|c|c|c|c|}
\hline $\begin{array}{c}\text { Model } \\
(\text { Coefficient })\end{array}$ & $\begin{array}{c}\text { Dependent } \\
\text { variable } \\
\left(\alpha_{0}\right)\end{array}$ & $\begin{array}{c}\text { Period } \\
\left(\beta_{0}\right)\end{array}$ & $\begin{array}{c}\text { Age } \\
\left(\alpha_{1}\right)\end{array}$ & $\begin{array}{c}\text { Period } \\
\text { x Age } \\
\left(\beta_{1}\right)\end{array}$ & $\begin{array}{c}\text { Age }^{2} \\
\left(\alpha_{2}\right)\end{array}$ & $\begin{array}{c}\text { Period } \\
\mathrm{x} \mathrm{Age}^{2} \\
\left(\beta_{2}\right)\end{array}$ & $\begin{array}{c}\text { Month } \\
\left(\alpha_{3}\right)\end{array}$ & $\begin{array}{c}\text { Period } \\
\text { x Month } \\
\left(\beta_{3}\right)\end{array}$ & $\begin{array}{c}\text { Month }^{2} \\
\left(\alpha_{4}\right)\end{array}$ & $\begin{array}{c}\text { Period } \\
\text { x Month } \\
\left(\beta_{4}\right)\end{array}$ \\
\hline 1 & $\mathrm{CW}$ & & $\mathrm{Y}$ & & $\mathrm{Y}$ & & & & & \\
\hline 2 & $\mathrm{CW}$ & $\mathrm{Y}$ & $\mathrm{Y}$ & & $\mathrm{Y}$ & & & & & \\
\hline 3 & $\mathrm{CW}$ & $\mathrm{Y}$ & $\mathrm{Y}$ & $\mathrm{Y}$ & $\mathrm{Y}$ & $\mathrm{Y}$ & & & & \\
\hline 4 & $\mathrm{CW}$ & $\mathrm{Y}$ & $\mathrm{Y}$ & $\mathrm{Y}$ & $\mathrm{Y}$ & $\mathrm{Y}$ & $\mathrm{Y}$ & $\mathrm{Y}$ & & \\
\hline 5 & $\mathrm{CW}$ & $\mathrm{Y}$ & $\mathrm{Y}$ & $\mathrm{Y}$ & $\mathrm{Y}$ & $\mathrm{Y}$ & $\mathrm{Y}$ & $\mathrm{Y}$ & $\mathrm{Y}$ & $\mathrm{Y}$ \\
\hline 6 & $\mathrm{Log} \mathrm{CW}$ & $\mathrm{Y}$ & $\mathrm{Y}$ & $\mathrm{Y}$ & $\mathrm{Y}$ & $\mathrm{Y}$ & $\mathrm{Y}$ & $\mathrm{Y}$ & & \\
\hline 7 & $\mathrm{Log} \mathrm{CW}$ & $\mathrm{Y}$ & $\mathrm{Y}$ & $\mathrm{Y}$ & $\mathrm{Y}$ & $\mathrm{Y}$ & $\mathrm{Y}$ & $\mathrm{Y}$ & $\mathrm{Y}$ & $\mathrm{Y}$ \\
\hline
\end{tabular}

Table 2.3: Summary of regression model variables

The core models use cost weight as the dependent variable. Two models use the log of cost weight, as explained above on page 23. The main variable of interest is the period variable and its interaction terms. We use the period variable, and period interaction variables in richer models, to test the hypothesis that the reforms were independently associated with an increase in costs. 
The first model focuses on the influence of age on cost weight. It does not attempt to isolate the effect of the reforms; it does not include the period or background time trend variables. The regression equation for this model, named Model 1, is:

$$
C W=\alpha_{0}+\alpha_{1} a g e+\alpha_{2} a g e^{2}
$$

Richer models include the period variable and control for a greater range of potential confounding variables. Model 4, for example, controls for age and time trend variables as well as allowing for interaction effects with the period variable. The regression equation for this model is:

$$
\begin{aligned}
C W= & \alpha_{0}+\alpha_{1} \text { age }+\alpha_{2} \text { age } e^{2}+\alpha_{3} \text { mont } h+\alpha_{4} \text { mont } h^{2}+\beta_{0} \text { period }+\beta_{1} \text { period } \times \text { age } \\
& +\beta_{2} \text { period } \times \text { age } e^{2}+\beta_{3} \text { period } \times \text { mont } h+\beta_{4} \text { period } \times \text { mont } h^{2}
\end{aligned}
$$

An alternative time trend model, Model 1a, uses a binary variable for each month. This alternative approach is a semi-parametric technique, which does not impose a particular structural form on the time trend variable. The coefficients for each month are estimated separately; collectively they suggest the pattern between the predicted cost weight and a background time trend.

\subsection{Results}

This section provides summary statistics as a backdrop to the econometric analysis.

\subsubsection{Summary statistics}

The full data set spanning 1 July 1988 to 30 June 1999 contains 6,619,761 records, as shown in Table 2.4 on page 27. The main analysis focuses on the seven years spanning 1 July 1988 to 30 June 1995, which provides $3,736,814$ records.22

\footnotetext{
${ }^{22}$ While we analyse the data by unit record (an individual discharge record), given the large number of observations the figures below present the data using monthly averages.
} 
CHEs were established on 1 July 1993. This date is used to divide the data into two periods: operations performed before 1 July 1993 and those after the reforms. There are $2,510,896$ observations (67.2 percent of the total number of observations) for the five years before the reforms and 1,225,918 (32.8 percent) observations for the two years after the reforms ${ }^{23}$ The following tables report descriptive statistics on the full data set, according to whether the publicly-funded procedure was performed in a public or private hospital.

Table 2.4 indicates the dominance of public hospital provision. Just over 1.1 percent of the full data set $(75,300$ records) were publicly-funded procedures performed in private hospital facilities. The proportion of private procedures rose from 0.52 percent between 1988 to 1993 to 1.54 percent in the six years following the reforms. This primarily reflects the funding injections in 1995 and 1997 to reduce hospital waiting lists. 24

\begin{tabular}{|l|r|r|r|r|}
\hline & \multicolumn{4}{|c|}{ Number of observations } \\
& $\begin{array}{l}\text { July 1988 } \\
\text { - June 1993 }\end{array}$ & $\begin{array}{c}\text { July 1993 } \\
\text { - June 1995 }\end{array}$ & $\begin{array}{c}\text { July 1995 } \\
\text { - June 1999 }\end{array}$ & Total \\
\hline Publicly-performed & $2,510,896$ & $1,225,918$ & $2,633,934$ & $6,370,748$ \\
\hline Privately-performed & 13,048 & 5,636 & 55,687 & 74,371 \\
\hline Filtered records & 90,127 & 36,246 & 48,269 & 174,642 \\
\hline Total & $2,614,071$ & $1,267,800$ & $2,737,890$ & $6,619,761$ \\
\hline
\end{tabular}

Table 2.4: Number of observations

Table on page 28 shows that the cost of treatment had a wide range. The distribution shows that the majority of cases were low to average cost, a moderate number of expensive cases and a small number of extremely expensive cases. Just under one half (47.0 percent) of the cases had a cost weight between 0 and 0.5 , just over one quarter (26.0 percent) were between 0.5 and 1.0, and just under one quarter (23.8 percent) were between 1.0 and 5.5. Less than 2.8 per cent of cases had a cost weight of greater than or equal to 5.5.

\footnotetext{
${ }^{23}$ There are a further 2,633,934 records between 1 July 1995 and 30 June 1999.

${ }^{24}$ The proportion of private procedures fell to 0.46 percent between 1993 and 1995 and rose to 2.07 percent between 1995 and 1999.
} 


\begin{tabular}{|c|c|c|c|c|c|c|}
\hline \multirow[b]{2}{*}{ Cost weight } & \multicolumn{3}{|c|}{ Publicly-performed } & \multicolumn{3}{|c|}{ Privately-performed } \\
\hline & $\begin{array}{c}\text { July '88 } \\
\text { - June '93 }\end{array}$ & $\begin{array}{l}\text { July '93 } \\
\text { - June '95 }\end{array}$ & $\begin{array}{l}\text { July '95 } \\
\text { - June '99 }\end{array}$ & $\begin{array}{c}\text { July '88 } \\
\text { - June '93 }\end{array}$ & $\begin{array}{l}\text { July '93 } \\
\text { - June '95 }\end{array}$ & $\begin{array}{l}\text { July '95 } \\
\text { - June '99 }\end{array}$ \\
\hline Average & 1.4519 & 1.1883 & 1.135 & 4.7548 & 3.8778 & 4.3003 \\
\hline Minimum & 0.0543 & 0.0543 & 0.0543 & 0.1792 & 0.1792 & 0.1337 \\
\hline Maximum & 159.7824 & 154.1508 & 152.3087 & 113.1109 & 104.7685 & 110.0087 \\
\hline Std. dev. & 4.7368 & 3.6789 & 3.1093 & 11.4875 & 10.4629 & 12.5777 \\
\hline
\end{tabular}

Table 2.5: Cost weight statistics (filtered data set, $\mathrm{n}=6,370,748$ )

Table 2.6 indicates that the age of patients in public hospitals was below that of patients treated in private hospitals on average, but generally had greater variation.

\begin{tabular}{|c|c|c|c|c|c|c|}
\hline \multirow[b]{2}{*}{ Age } & \multicolumn{3}{|c|}{ Publicly-performed } & \multicolumn{3}{|c|}{ Privately-performed } \\
\hline & $\begin{array}{c}\text { July '88 } \\
\text { - June '93 }\end{array}$ & $\begin{array}{l}\text { July '93 } \\
\text { - June '95 }\end{array}$ & $\begin{array}{l}\text { July '95 } \\
\text { - June '99 }\end{array}$ & $\begin{array}{l}\text { July '88 } \\
\text { - June '93 }\end{array}$ & $\begin{array}{l}\text { July '93 } \\
\text { - June '95 }\end{array}$ & $\begin{array}{c}\text { July '95 } \\
\text { - June '99 }\end{array}$ \\
\hline Average & 38.3 & 37.0 & 39.5 & 47.4 & 51.4 & 41.5 \\
\hline Minimum & 0 & 0 & 0 & 0 & 0 & 0 \\
\hline Maximum & 118 & 107 & 112 & 100 & 99 & 106 \\
\hline Std. dev. & 26.3 & 27.7 & 27.9 & 26.1 & 24 & 29.3 \\
\hline
\end{tabular}

Table 2.6: Age statistics (filtered data set, $\mathrm{n}=6,370,748$ )

\section{Cost weight outliers}

Cost weights greater than 2.13 (727,997 cases or 11.0 percent of the records between 1988 to 1999) were identified as outliers based on the test in Clark \& Randal (2004). Supplementary econometric analyses showed that excluding outliers did not affect the main qualitative results related to the period variables, that is, the algebraic signs of the coefficients and statistical significance of the period estimates did not change. Appendix A.1.4 reports on the supplementary regression results with the outliers removed.

As the outliers were high cost cases, removing them lowered the overall average cost weight. These outlier cases were disproportionately associated with very young and older 
people. Removing these cases changed the algebraic signs of the estimated coefficients for the age variable in some models.

The main analyses below do not remove the outliers. These high cost cases may have been of interest to the reformers, and as a focus for the newly installed CEOs they may also have been a source of tension between managers and specialists.

\section{Public and private provision of publicly-funded procedures}

Table 2.7 shows the average cost weight and age before and after the reforms and the break due to the coding and funding injections. The data set excludes cases with a zero cost weight. It decomposes the statistics according to whether a publicly-funded procedure was performed in a public or a private hospital. The cost weight statistics in this table are not adjusted for any potentially confounding influences before and after the reforms, such as changes in the age distribution.

\begin{tabular}{|c|c|c|c|c|c|c|}
\hline & \multirow{2}{*}{$\begin{array}{c}\text { Before } \\
(1988-93)\end{array}$} & \multirow{2}{*}{$\begin{array}{c}\text { After } \\
(1993-95)\end{array}$} & \multirow{2}{*}{$\begin{array}{c}\text { Post breaks } \\
(1995-99)\end{array}$} & \multicolumn{2}{|c|}{ Change } \\
\hline & & & & & Before-After & Before-Post \\
\hline \multirow{3}{*}{$\begin{array}{c}\text { Cost } \\
\text { weight }\end{array}$} & - public & 1.4519 & 1.1883 & 1.135 & -0.2635 & -0.3169 \\
\hline & - private & 4.7548 & 3.8778 & 4.3003 & -0.877 & -0.4545 \\
\hline & - total & 1.4689 & 1.2006 & 1.2005 & -0.2683 & -0.2684 \\
\hline \multirow{3}{*}{ Age } & - public & 38.3 & 37.0 & 39.5 & -1.3 & 1.2 \\
\hline & - private & 47.4 & 51.4 & 41.5 & 4.0 & -5.9 \\
\hline & - total & 38.4 & 37.1 & 39.5 & -1.3 & 1.2 \\
\hline
\end{tabular}

Table 2.7: Change in average cost weight and age over the reform period

Table 2.7 indicates that the average cost of a procedure fell following the reforms across both hospital types, by -0.2683 or approximately 18.3 percent. The table also shows that the average age of patients fell following the reforms. The additional patients were likely to be younger with less complex conditions, lowering the average age and complexity of cases. Further, following the waiting time funding injections in 1995 and 1997, the 
average age of patients treated rose in public facilities and fell in private facilities.${ }^{25}$ These features of the data suggest that controlling for changes in the age distribution and for the funding injections is important, in order to separate the influence of these changes from those of the reforms.

Figure 2.1 shows the distribution of cost weighted discharges for the sample period as a whole, excluding cases with a zero cost weight. The figure indicates that the majority of cases have a cost weight below the normalised average of 1.0. As noted above, this reflects a skewed distribution with a large number of low to average cost cases, a moderate number of mid-range costing cases and a small number of extremely expensive cases.

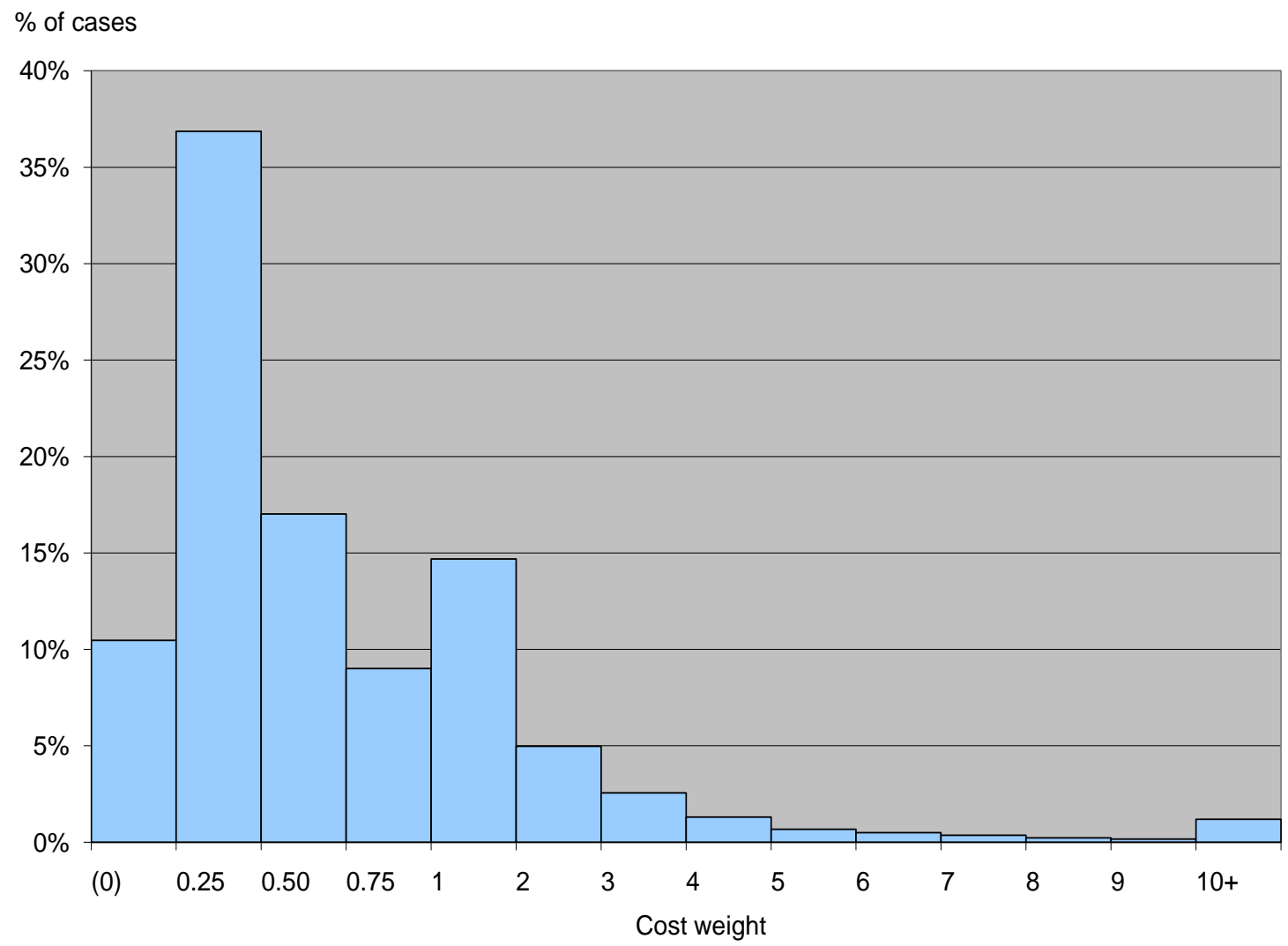

Figure 2.1: Relative frequency of cost weighted discharges

\footnotetext{
${ }^{25}$ The additional procedures permitted by the funding injections tended to be provided in private facilities. This is reflected in the greater fall in the average cost weight and age in private facilities versus public facilities. The likely impacts of the funding injections are discussed further on page 33.
} 


\section{The pattern of cost weight over time}

Figures 2.2 and 2.3 on page 32 show the pattern of cost weight over time, using the monthly average of cost weight ${ }^{26}$ The first figure plots the average cost weight for publicly-treated patients, and the second figure is for publicly-funded privately-treated patients.

The average cost of patients treated in public hospitals was lower than publicly-funded privately-treated patients. However, public hospitals tended to perform the most complex and high risk surgeries, in addition to a large volume of low cost procedures.

Figure 2.2 exhibits a downward trend in the average cost weight per month of publiclyperformed procedures. There is a modest increase in average cost immediately after the reforms in July 1993, followed by a drop and a moderately increasing trend, before a return to a falling trend after July 1995.

Figure 2.3 also shows a moderate downward trend in the average cost weight per month for publicly-funded privately-performed procedures. The average cost weight for privatelyperformed procedures fell after the reforms (see Table 2.7). There is a marked drop in the average cost weight for privately-performed procedures after July 1995 followed by an increase after July 1997. These changes correspond to the two funding injections aimed at reducing waiting times for publicly-funded services.

The initial fall after July 1995 is likely to reflect an increase in the volume of relatively simple procedures that were on waiting lists, but could not be treated in the capacityconstrained public system. The later increase in the average cost weight is likely to involve a shift of patients with more complex needs who would previously have been treated in public hospitals. This is consistent with the slight fall in the average cost in public hospitals in the period after July 1997, which is discernable in Figure 2.2, and a rise for private hospitals, as in Figure 2.3.

\footnotetext{
${ }^{26} \mathrm{It}$ is not practical to plot individual cost weights over time due to the large number of data points.
} 


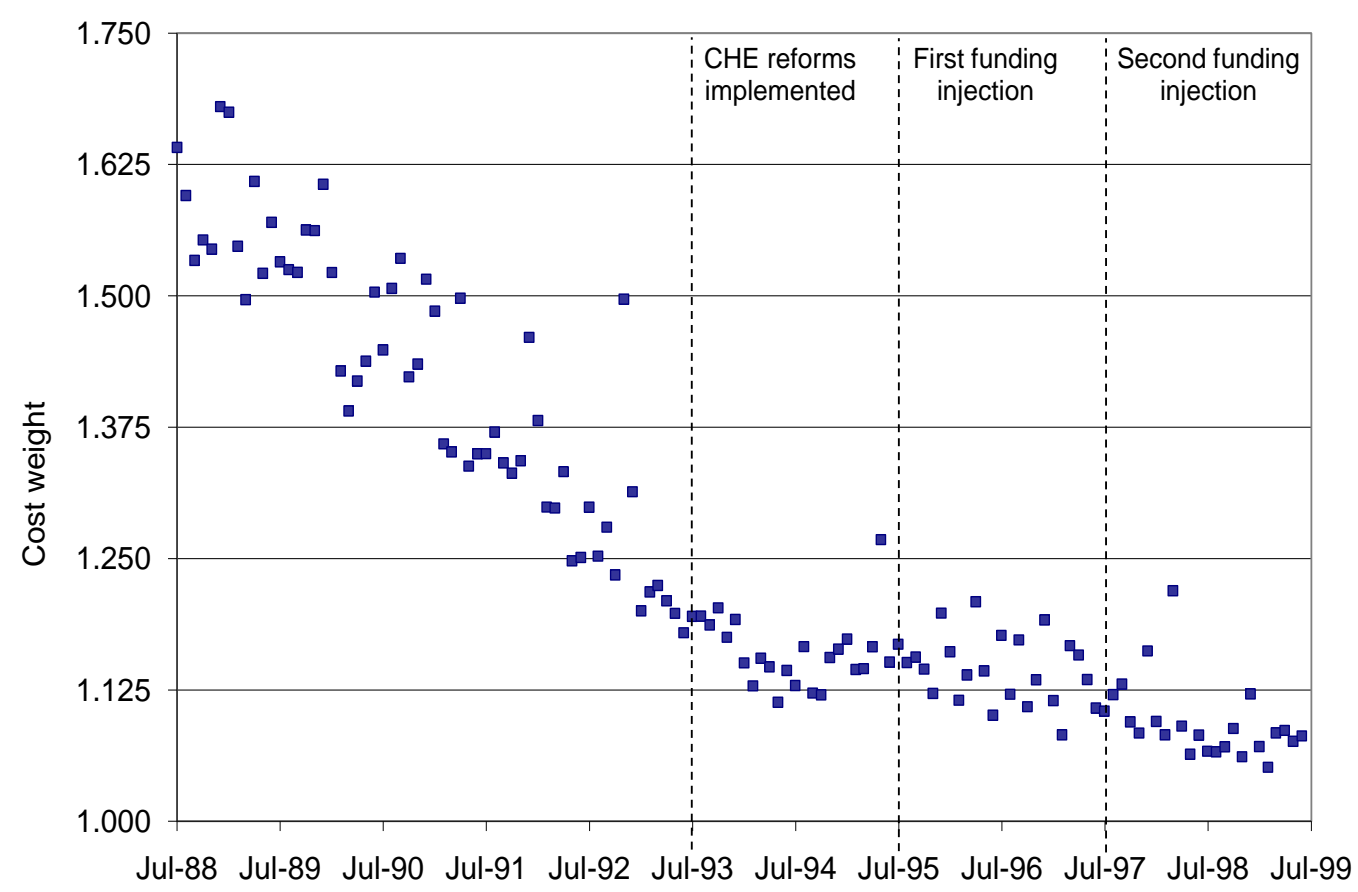

Figure 2.2: Average cost weight of publicly-performed procedures over time

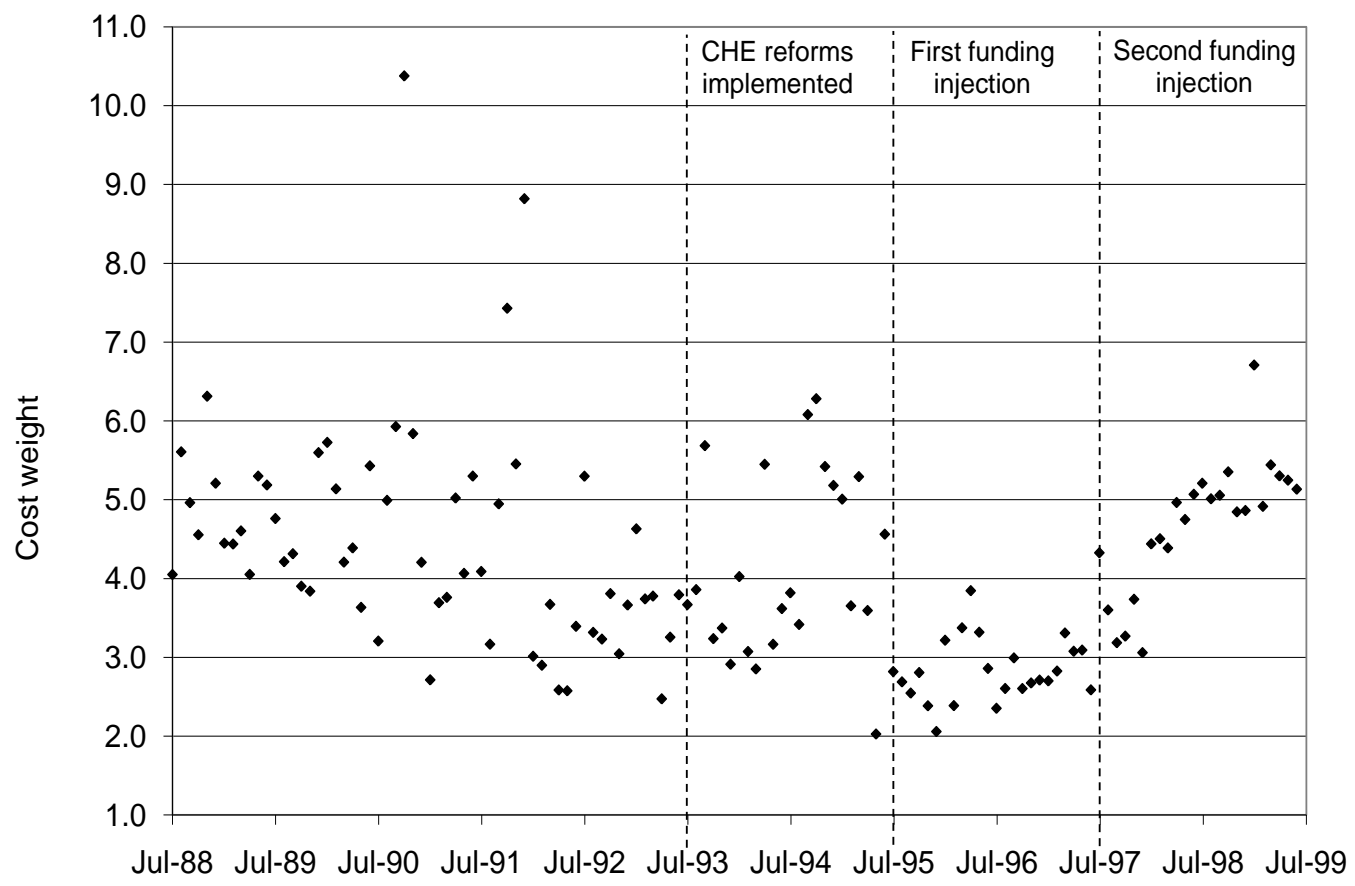

Figure 2.3: Average cost weight of privately-performed procedures over time 


\section{The frequency of operations by sector}

Figure 2.4 shows the number of publicly-funded operations performed in the public and private sectors over the timeframe. The number of publicly-performed procedures and the total are an order of magnitude greater than privately-performed procedures, and the number of these operations is on the right-hand side axis.

Figure 2.5 shows a growing proportion of privately-performed procedures after 1995. This is likely to be due to funding injections in July 1995 and July 1997.

\section{Funding injections to reduce waiting lists}

Publicly-funded elective services in New Zealand are rationed, typically resulting in a waiting lists for some services. Until 1998, waiting lists were managed by individual surgeons who assessed a patient's priority ${ }^{27}$ Funding injections in 1995 and 1997 were motivated by concerns over the length of time people were waiting for some types of surgery and were aimed at reducing average waiting times.

Against a background trend of a decreasing number of operations per month, the proportion of privately-performed procedures increased substantially after the 1995 funding injection, as shown in Figures 2.4 and 2.5 below 28 The funding injections lowered the cost of treating the average case (see Table 2.7).

\footnotetext{
${ }^{27}$ The Ministry of Health implemented a booking system based on a set of centrally-developed priority criteria in 1998, which stemmed from concerns over the equity and effectiveness of the prioritisation decisions of individual surgeons (Fraser, Alley \& Morris 1993, Hadorn \& Holmes $1997 a$, Hadorn \& Holmes 1997b, Deloitte et al. 1998).

${ }^{28}$ This may have reflected capacity constraints on public facilities and competition amongst providers as part of the broader health sector reforms, which increased the use of private facilities. However, there appears to have been some relocation of existing public hospital surgery volumes to private hospitals, rather than the funding leading to a pure increase in total number of procedures.
} 


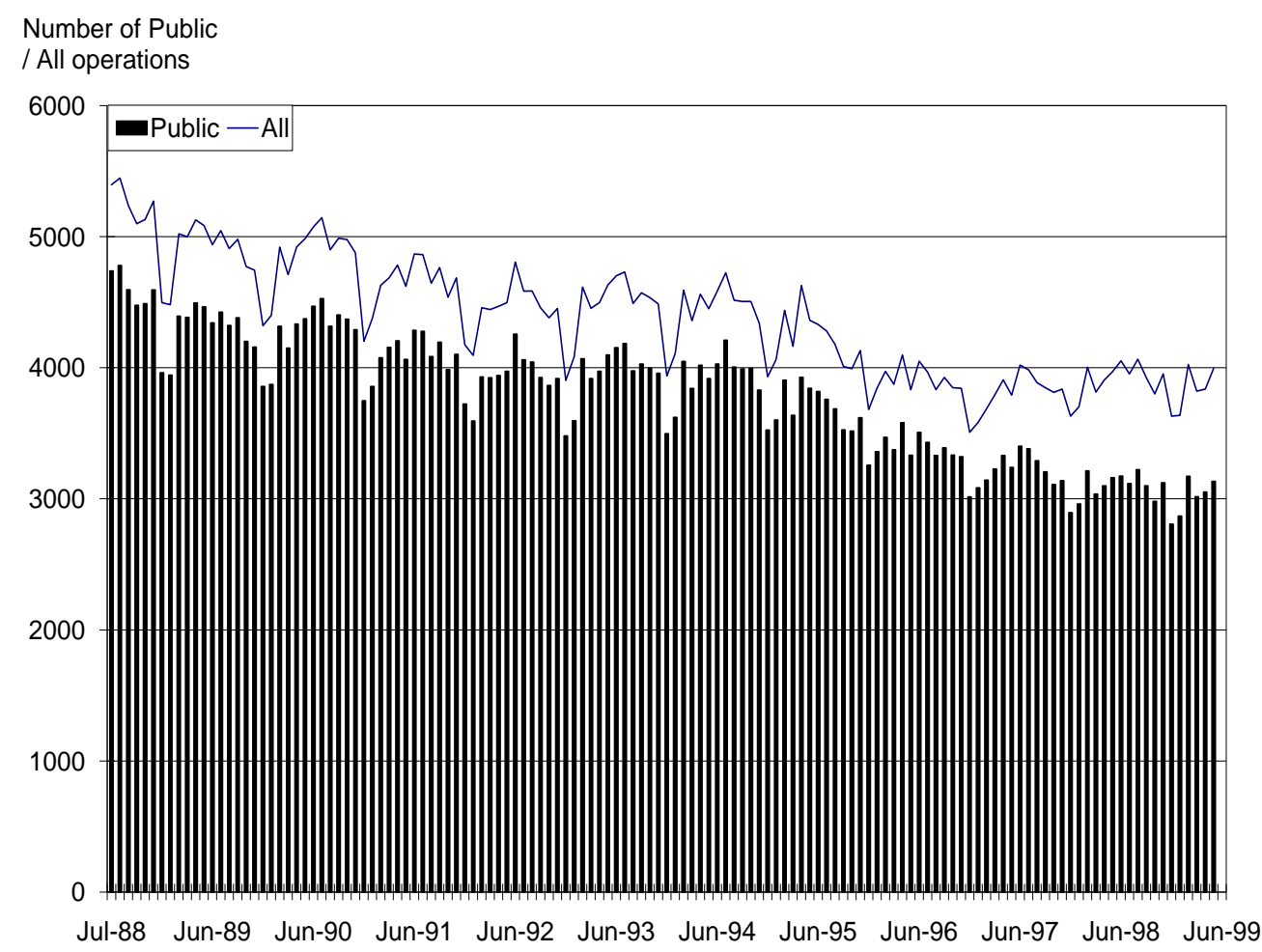

Figure 2.4: Number of publicly-performed procedures

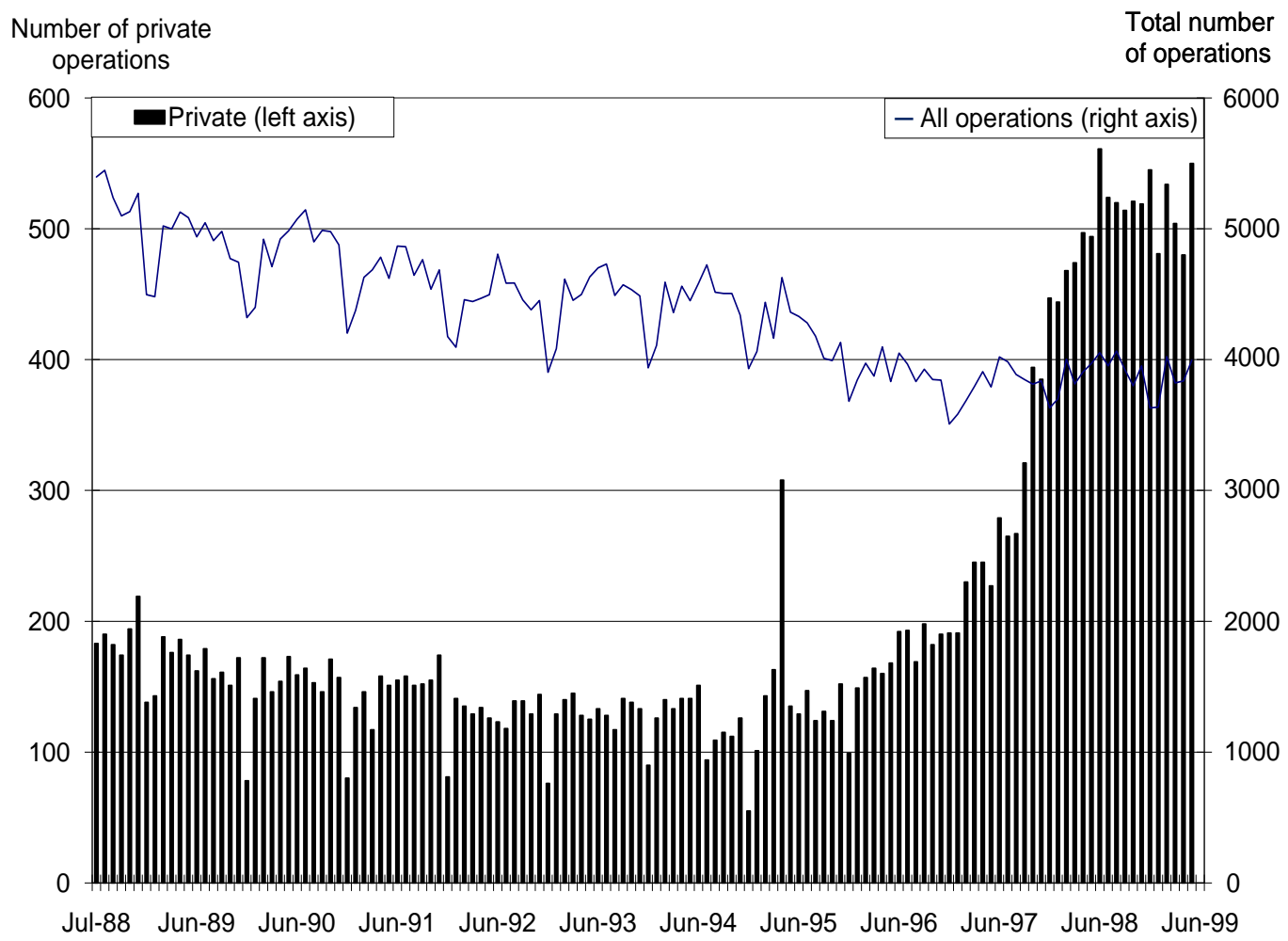

Figure 2.5: Number of privately-performed procedures 
The injections allowed people to be treated whose conditions had previously been assessed as being of insufficient priority for treatment. The average age of new patients fell, which may also correspond to the new patients having both less severe conditions. Both lower severity and younger age are likely to have contributed to the lower average cost weight reported in the later years of the data set. The econometric analysis isolates the funding and age effects to identify the independent effect of the CHE reforms on treatment costs.

\section{Age}

Figure 2.6 shows the unadjusted relationship between age and cost weight.

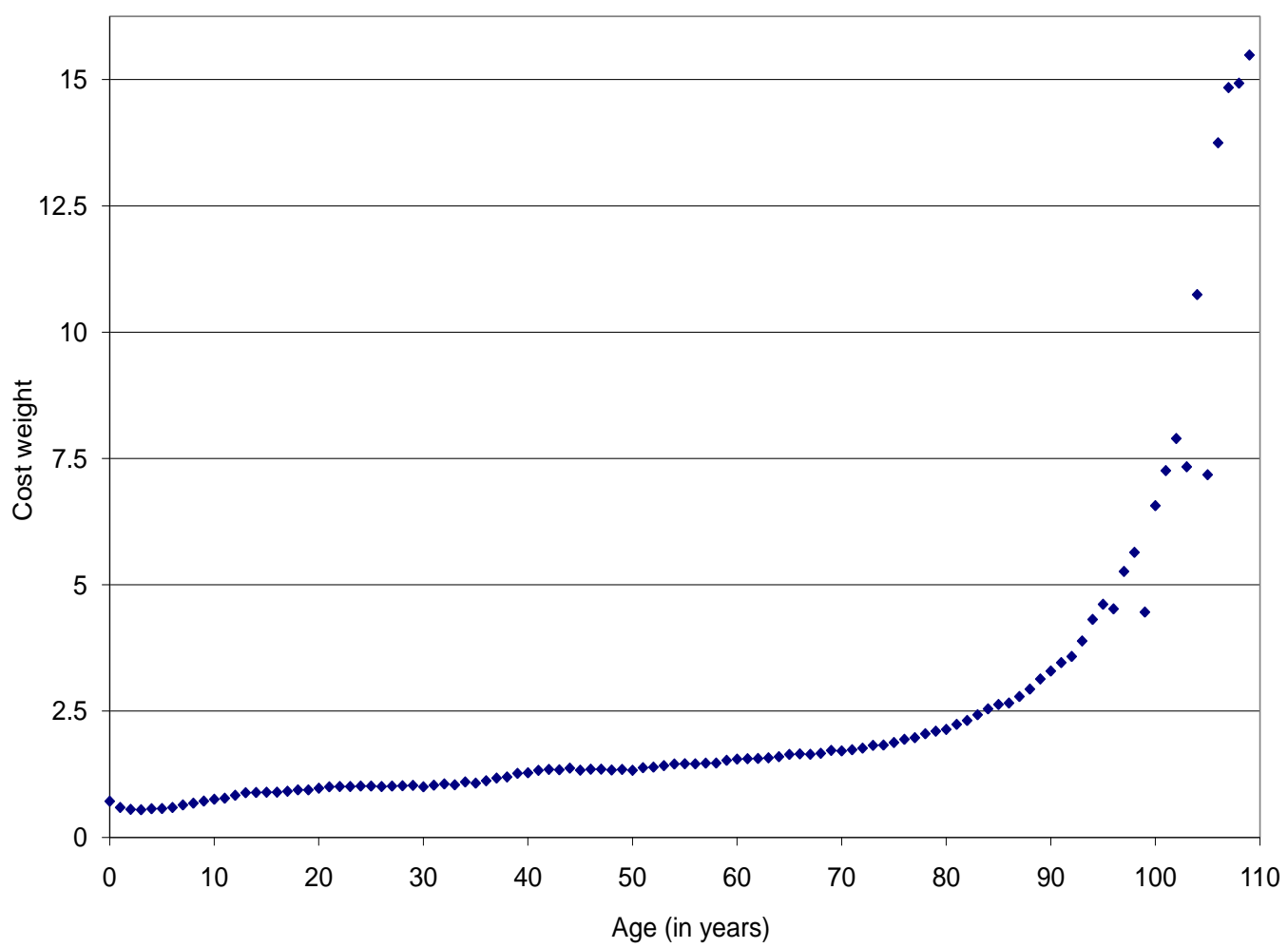

Figure 2.6: Relationship between age and cost weight

Average cost weight declines in early childhood followed by an increase with age. The pattern becomes less clear after 90 years of age. This is in part due to the dwindling number of people in the data set at older ages.

The average age of publicly-funded patients fell in the post-reform period, as shown in Table 2.7 on page 29. As age is correlated with cost weight, this would tend to lead to lower 
cost weights over time. Therefore, we include age-related variables in the econometric analysis to isolate the effects of age on cost from any reform-related effects.

\subsubsection{Econometric analysis}

This section presents the results of the econometric analysis using the data described above. After isolating the effects of changes in the age distribution and a background time trend, we examine the independent effect of the reforms on treatment costs.

\section{Summary of regression results}

Table 2.3 in section 2.4 describes the econometric model specifications. Table 2.8 shows the estimated regression coefficients for these models. Table A.2 in appendix A.1.3 expands Table 2.8 to show the standard errors and p-values.

\begin{tabular}{|c|c|c|c|c|c|c|c|c|c|c|}
\hline $\begin{array}{c}\text { Model } \\
\text { (Coefficient) }\end{array}$ & $\begin{array}{c}\text { Intercept } \\
\left(\alpha_{0}\right)\end{array}$ & $\begin{array}{c}\text { Period } \\
\left(\beta_{0}\right)\end{array}$ & $\begin{array}{l}\text { Age } \\
\left(\alpha_{1}\right)\end{array}$ & $\begin{array}{l}\text { Period } \\
\text { x Age } \\
\left(\beta_{1}\right)\end{array}$ & $\begin{array}{l}\mathrm{Age}^{2} \\
\left(\alpha_{2}\right)\end{array}$ & $\begin{array}{l}\text { Period } \\
\text { x Age } \\
\left(\beta_{2}\right)\end{array}$ & $\begin{array}{c}\text { Month } \\
\left(\alpha_{3}\right)\end{array}$ & $\begin{array}{c}\text { Period } \\
\text { x Month } \\
\left(\beta_{3}\right)\end{array}$ & $\begin{array}{l}\text { Month }^{2} \\
\left(\alpha_{4}\right)\end{array}$ & $\begin{array}{c}\text { Period } \\
\text { x Month }{ }^{2} \\
\left(\beta_{4}\right)\end{array}$ \\
\hline 1 & $0.7768^{\ddagger}$ & & $0.0034^{\frac{1}{\ddagger}}$ & & $0.0002 \ddagger$ & & & & & \\
\hline 2 & $0.8758^{\ddagger}$ & $-0.2560 \ddagger$ & $0.0023^{\ddagger}$ & & $0.0002 \ddagger$ & & & & & \\
\hline 3 & $0.8626 \ddagger$ & $-0.2179 \ddagger$ & -0.0005 & $0.0090 \ddagger$ & $0.0003 \ddagger$ & $-0.0002 \ddagger$ & & & & \\
\hline 4 & $1.1069 \ddagger$ & $-0.3369 \ddagger$ & 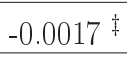 & $0.0103 \ddagger$ & $0.0003 \ddagger$ & $-0.0002 \ddagger$ & $-0.007 \ddagger$ & $0.0053 \stackrel{\ddagger}{\ddagger}$ & & \\
\hline 5 & $1.1074^{\ddagger}$ & $1.8490 \stackrel{\ddagger}{\ddagger}$ & 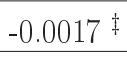 & $0.0103 \ddagger$ & $0.0003^{\ddagger}$ & $-0.0002 \stackrel{\ddagger}{\ddagger}$ & $-0.0071^{\ddagger}$ & $-0.0555^{\ddagger}$ & 0.000001 & $0.0004 \stackrel{+}{\dagger}$ \\
\hline 6 & $-0.2552 \ddagger$ & $-0.0886 \ddagger$ & $0.0016^{\ddagger}$ & $0.0019 \ddagger$ & $0.00004 \ddagger$ & $-0.00003 \ddagger$ & $-0.0015^{\ddagger}$ & $0.0013^{\ddagger}$ & & \\
\hline 7 & $-0.2561^{\ddagger}$ & $0.2171^{\ddagger}$ & $0.0016 \stackrel{\ddagger}{\ddagger}$ & $0.0019 \ddagger$ & $0.00004^{\ddagger}$ & $-0.00003^{\ddagger}$ & $-0.0015^{\ddagger}$ & $-0.0073^{\ddagger}$ & -0.000001 & $0.0001 \ddagger$ \\
\hline
\end{tabular}

Table 2.8: Summary of regression model results

In general, the variables examined tended to be highly statistically significant. Table 2.8 shows that in all the models, except the simple Model 1, the period variable is both independently significant and jointly significant with the other period interaction terms.

Due to the large sample size, the estimated standard errors tend to be very small. This means that even small changes tend to pass conventional significance tests using t-values and p-values. However, we find that the estimated coefficients for the reform variables 
remain statistically significant using various model and dependent variable forms. The estimated effects are substantial: our results, discussed below, show that the predicted average treatment cost per patient increases by between 11 percent and 14.5 percent immediately after the reforms. Taken alongside the real concerns expressed in the criticisms of the CHE reforms, these results provide a motivation for the theoretical models, which are the primary focus of this thesis.

Below we consider the effect of the potential confounding variables. This analysis suggests that relationships with cost are as expected, and it is appropriate to include age and time trend variables in our model to control for these effects.

\section{Age effects}

Model 1 indicates that a patient's age is positively related to treatment cost. The coefficient on age is positive, contrary to our hypothesis that it would have a negative sign. However, the effects of the reforms on how patients of different ages are treated may cloud this relationship, as the first two models do not include the interaction variables with age and period. Model 3 includes interaction terms between the age and period variables. The results from this model are consistent with the expected relationship between age and cost weight prior to the reforms.

Model 1 is a simple model that aims to control for the change in the age composition of the population over time. Plotting the estimated residuals from Model 1 gives a sense of the underlying pattern in the data after controlling for the influence of age ${ }^{29}$ Figure 2.7 plots the monthly average of the residuals from Model 1; the corresponding model equation is noted below the figure.

Figure 2.7 shows that, after accounting for changes in the age composition over the timeframe examined, there remains a time-related pattern in the cost weight variable. The

\footnotetext{
${ }^{29}$ Residuals are the difference between the predicted cost weight and actual cost weight for each discharge record. A residual reflects how much of an actual observation is explained by the model's prediction. An obvious pattern in a residual plot can diagnose omitted variables or an incorrectly specified functional form of the model (Gujarati 1995, 461).
} 
figure indicates a strong negative trend over time. This suggests that while age explains some of the behaviour of the cost weight variable over time, it leaves much unexplained. The next section explores some further possible explanatory variables and presents the results of including time-related influences on cost weight behaviour.

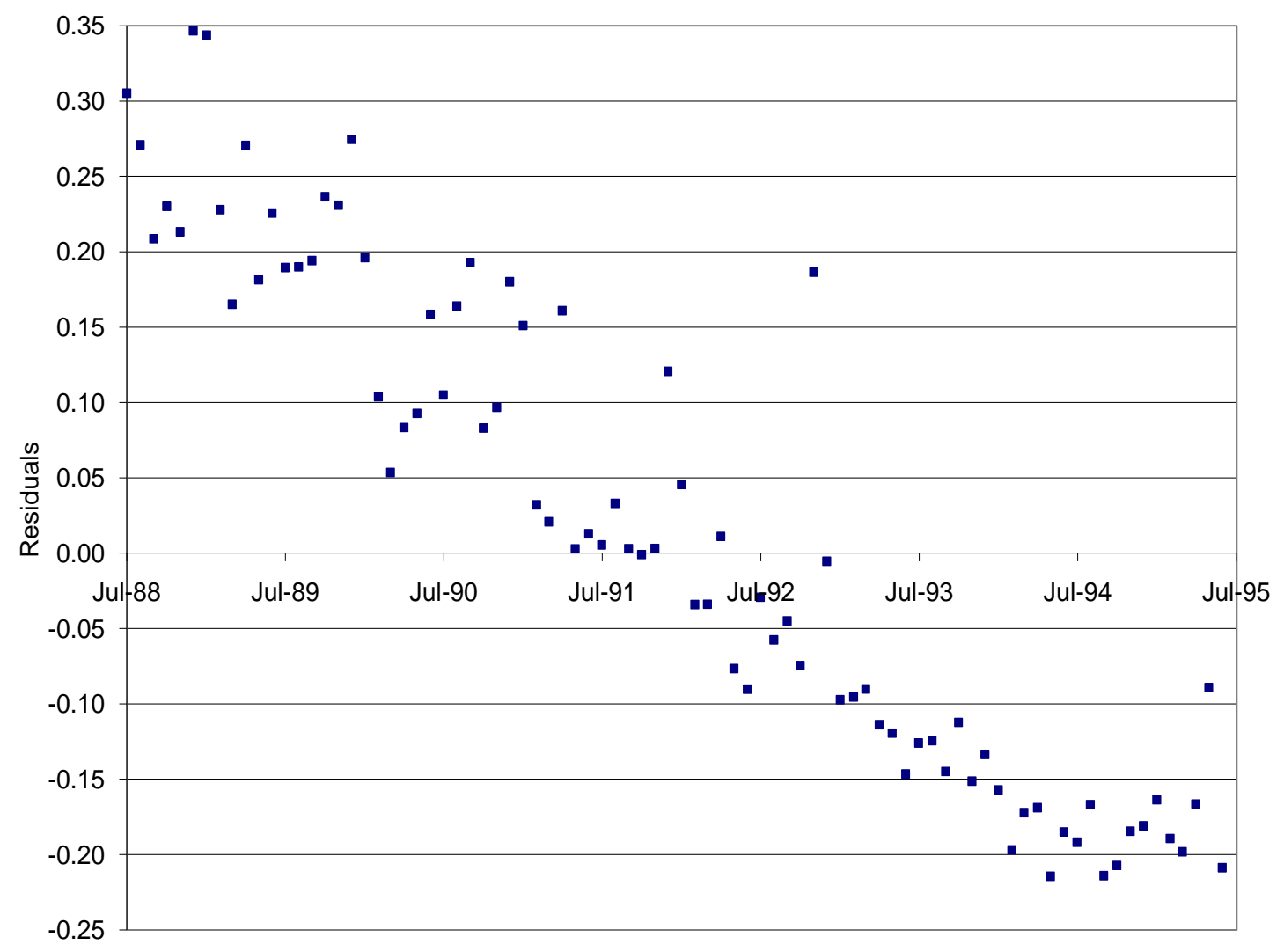

Figure 2.7: Residual plot of Model 1

$$
C W=\alpha_{0}+\alpha_{1} a g e+\alpha_{2} a g e^{2}
$$

\section{Time effects}

It is likely that non-reform related changes, for example improvements in medical technology, could cause costs to change over time. We include both reform and time trend variables to isolate the effect of the reforms from a background time trend.

Model 2 adds the period variable to Model 1 as a first step to identify the effect of the reforms. Model 3 allows for an interaction between the age and period variables. The 
highly statistically significant negative coefficient of -0.2560 for the period variable from Model 2 suggests a fall in costs after the reforms compared to the period before the reforms. However, the residual plot in Figure 2.7 suggests that the average cost weight follows a negative trend that is not directly related to the reforms.

Model 1a adapts Model 1 to include a set of binary variables for the month of discharge

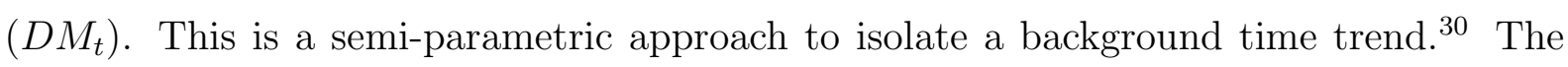
semi-parametric approach used in Model 1a does not impose any linearity constraint on the trend.

Figure 2.8 plots the cost weights predicted by Model 1a, holding age constant. The plotted points may be interpreted as the average cost weight for publicly-performed services in given month after removing the influence of age and changes in the age distribution over time. As age tends to have a positive relationship with the cost weight, removing the age effects shifts the entire distribution of average cost weights downwards relative to the raw pattern shown in figure 2.2 on page 32 .

Figure 2.8 shows that up to the reforms in July 1993, there is a downward trend in the cost weight variable. The trend up to the reforms appears linear. Extrapolating a linear trend is unrealistic, however, as it would predict that costs would continue to fall to zero and eventually become negative, which is nonsensical. Therefore, we believe a non-linear trend is conceptually appropriate 31

\footnotetext{
${ }^{30}$ This method requires a reference month to be excluded. The first month (DM1) was excluded, which acts as the reference month.

${ }^{31}$ One interpretation of the background trend in the cost weight variable before the reforms is that the data capture a (reasonably) linear portion of a non-linear trend. This may reflect a quadratic trend, for example, where reductions in cost may initially be substantial but over time the incremental cost reductions become smaller. We include a quadratic variable in the more sophisticated, parametric time trend models.
} 


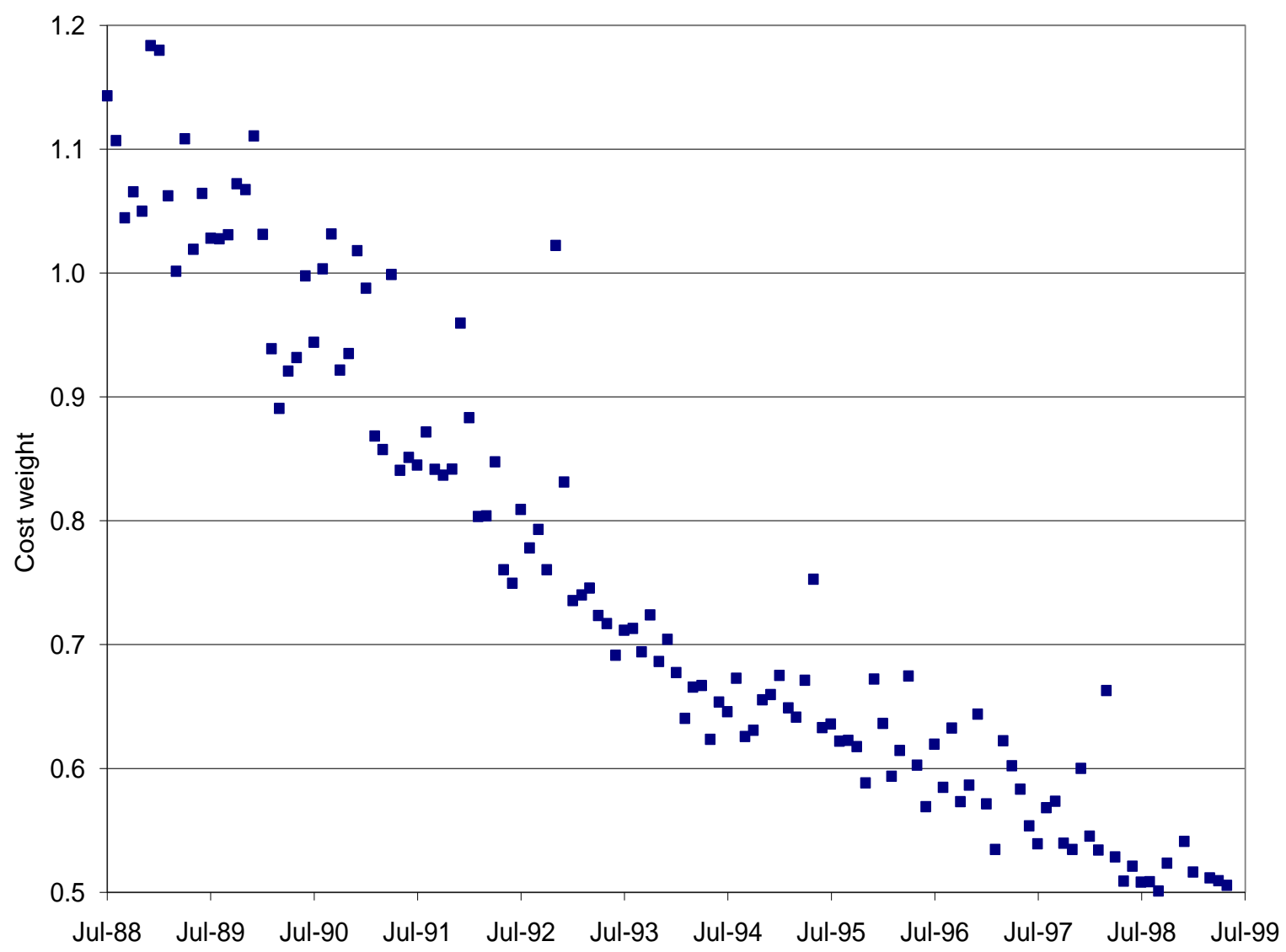

Figure 2.8: Semi-parametric model of cost weight and time (Model 1a)

$$
C W=\alpha_{0}+\alpha_{1} a g e+\alpha_{2} a g e^{2}+\delta_{i} D M_{i}, i \in\{2,132\}
$$

The second approach imposes a parametric form on the background time trend, which is simpler to interpret and is reasonable given the results of the semi-parametric investigation. Model 4 uses a linear time trend by including a discrete count variable called month; these two models differ in terms of the range of period interaction terms they include. Model 5 allows for a quadratic time trend by also including $m o n t h^{2}$.

The linear trend prior to the reforms is confirmed by the small coefficient on the non-linear element of the time trend, mont $h^{2}$, where it is included.

Models 4 and 5 also include interaction terms between age or age $e^{2}$ and period. These interaction terms capture the effect of the reforms on how patients of different ages were treated. 
Inclusion of the interaction term between age and period in models 4 and 5 yields the expected quadratic relationship in the period before the reforms, with a negative coefficient on age and a positive coefficient on age $e^{2}$. However, the reforms are associated with a change in this relationship. Combining the coefficient on age and the interaction term, period ${ }^{*}$ age, both models 4 and 5 indicate a positive relationship between age and treatment cost following the reforms.

This change may result from managers targeting high cost, young patients. The standard deviation of cost weight decreased from 4.66 before the reforms to 3.27 after the reforms, suggesting increased consistency of treatment. In this case, it is possible that very young, high cost patients were targeted to receive lower cost care while young to middle aged patients received more costly treatment than prior to the reforms. A decrease in the average treatment cost for very young patients and a disproportionate increase in the cost of treating young to middle age patients could replace the u-shaped age relationship seen prior to the reforms with a monotonically positive relationship.

The nature and distribution of the outlier cases also shed some light on the estimated change in the relationship between cost and age following the reforms. Section 2.5.1 noted that removing the outlier cases lowers the population's cost weight profile. Very young patients who had unusually high cost cases made up a disproportionately large part of this age group compared to the rest of the population. For example, people under 1 year of age with an outlier cost weight accounted for 4.83 percent of the discharges in this age group, but 41.6 percent of the total cost weights for this age group.

The proportion of cases with outlier cost weights across the population was 10.8 percent, which accounted for 54.7 percent of the sum of the cost weights. Targeting treatment costs for the disproportionately expensive very young age group could eliminate the expected quadratic relationship between cost and age. Further, if the reforms reduced information flow between doctors and managers, different types of patients may have received more homogenous treatment. 


\section{Reform effects}

After isolating the effects of changes in the age distribution and a background time trend there remain significant effects from the reforms on costs. We use two models to explain these effects: Models 4 and 5. The key factors in these models are the period variable and its interaction terms.

The reforms could cause a step-change in treatment cost, a change in the age-treatment profile or a change in the treatment cost trend. In modelling terms, including both period intercept and age or time trend interaction variables, allows the function to rotate without being fixed at the vertical intercept. Therefore, the effects of the reforms on costs cannot be assessed by just focusing on the sign of the estimated period intercept $\left(\beta_{0}\right)$. Evaluating the impact of the reforms requires examination of the joint effect of the reforms on the intercept, which is captured by $\beta_{0}$; on the age relationship as measured by $\beta_{1}$ and $\beta_{2}$; and on the time trend ( $\beta_{3}$ and $\beta_{4}$, depending on the form of the time trend).

Figure 2.9 on page 44 illustrates the estimated relationship between cost weight, a linear time trend and age-related interaction effects generated by Model 4 (holding the nonreform related age relationship constant).

Model 4 indicates that the reforms were associated with an immediate rise in cost weight immediately after the reforms. The linear model also shows that cost weight fell more slowly after the reforms than before them. Taking the intercept shift together with the change in the time trend, Model 4 predicts that the average cost weight would have been higher than in the absence of the reforms. The predicted cost weight in July 1993 is 1.1180, taking into account the effects of the reforms and given the average age of 36.35 in that month. In the absence of the reforms, the predicted value is 1.0064 . This indicates that the reforms were associated with a rise of 11.1 percent in the reported cost of treating an average patient, as measured by the cost weight variable.

It is possible that it took some time for the full effect of the reforms to manifest after the formal implementation date of 1 July 1993. A supplementary analysis censored three and 
six month periods immediately after the reforms ${ }^{32}$ Re-running the main models using censored data gave statistically significant coefficients that also predicted an increase in costs following the reforms.

For example, the estimates for Model 4-6, with a 6 month transition period removed, gave estimated time trend coefficients of $\alpha_{3}=-0.0067$ and $\beta_{3}=0.0080$, which implies a positive cost trend following the reforms, and the age interaction coefficients were $\beta_{1}=$ 0.0099 and $\beta_{2}=-0.0002$, which implies a positive impact given the age distribution following the reforms. The estimated intercept coefficients were $\alpha_{0}=-1.0882$ and $\beta_{0}=$ -0.5423. Taken together, the trend and age interaction effects outweigh the negative change in the intercept, so the joint effect of the reforms was to increase the average cost weight. This supplementary analysis does not provide strong evidence that there was a transition period before the full effects of the reforms manifested.

Model 5 posits a non-linear time trend. Figure 2.10 on page 44 plots the predicted cost weights using the estimated coefficients from Model 5, which has age interaction effects and a quadratic time trend, holding the non-reform related age relationship constant.

Model 5 predicts at any point after the reforms, the average cost weight would have been higher than in the absence of the reforms. This reflects the joint positive effects of a change in the intercept, the trend and age relationships resulting from the reforms. The estimated coefficients indicate that the reforms were associated with an immediate upward shift in the intercept, but a steepening in the trend immediately after the reforms. The increase in the intercept, however, outweighs the change in the trend, so the average cost weight increases immediately after the reforms. Given the average age of 36.35 in July 1993, the predicted cost weight is 1.1529 , but 1.0069 in the absence of the reforms, indicating a rise of 14.5 percent in the reported cost of treating an average patient due to the reforms. Furthermore, the downward trend softens after the reforms and, toward the end of 1994, the downward trend reverses and creates a rising trend in cost weight.

\footnotetext{
${ }^{32}$ Appendix A.1.5 reports the regression results.
} 


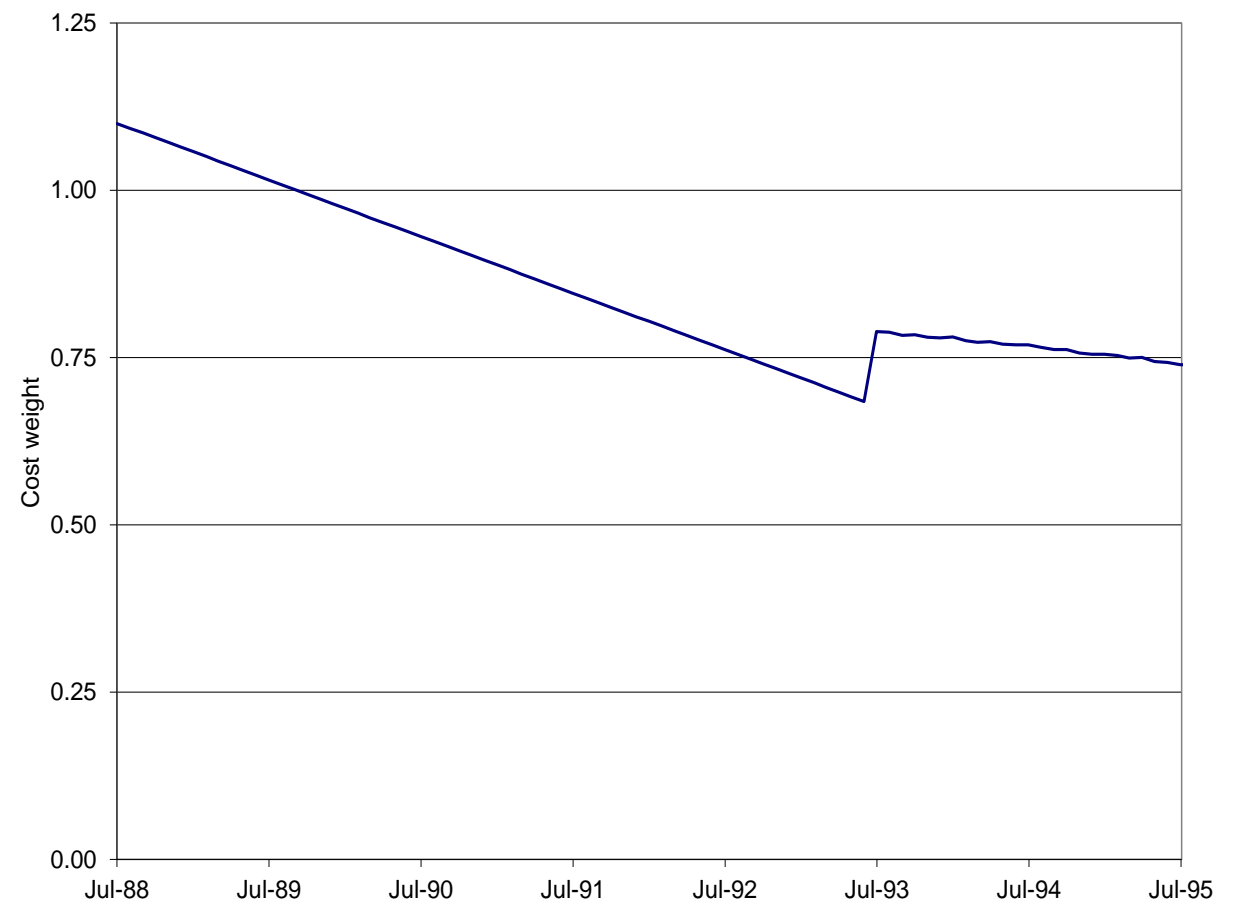

Figure 2.9: Model of cost weight including a linear time trend (Model 4)

$C W=\alpha_{0}+\beta_{0}$ period $+\alpha_{1}$ age $+\beta_{1}$ period $*$ age $+\alpha_{2}$ age $e^{2}+\beta_{2}$ period $*$ age ${ }^{2}+\alpha_{3}$ month $+\beta_{3}$ period $*$ month

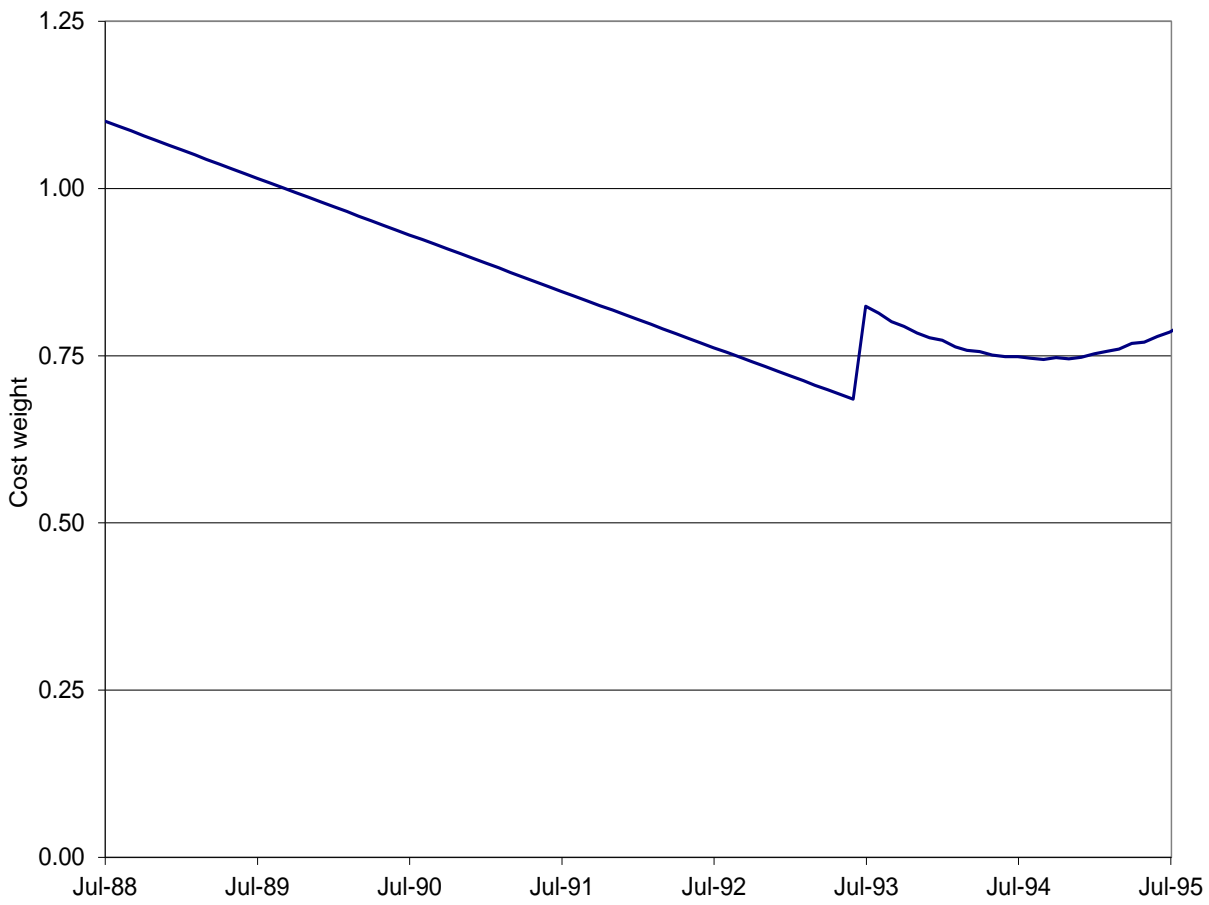

Figure 2.10: Model of cost weight including a quadratic time trend (Model 5)

$C W=\alpha_{0}+\beta_{0}$ period $+\alpha_{1}$ age $+\beta_{1}$ period $*$ age $+\alpha_{2}$ age ${ }^{2}+\beta_{2}$ period $*$ age $e^{2}+\alpha_{3} \operatorname{month}+\beta_{3}$ period $*$ month $+\alpha_{4}$ month ${ }^{2}+\beta_{4}$ period $*$ mont $h^{2}$ 
Both Model 4 and Model 5 estimate an immediate and substantial increase in the average treatment cost following the reforms. These models also indicate that the reforms altered the background trend in the behaviour of cost weights: costs fell less quickly after the reforms in the linear trend model, and eventually rise in the quadratic model. The net effect immediately after the reforms takes into account the direct impact (which affects the intercept) and indirect impacts on the time trend and age interaction effects (which affect the slope of the function). In the case of Model 5, the net effect is positive despite the initially steeper downward trend, and ultimately the trend rises. Both models predict a higher cost weight at any point in time following the reforms independent of other influences.

Models 4 and 5 explain much of the pattern in the cost weight data: the impact of the reforms, age and a background time trend are jointly highly statistically significant. Figures 2.11 and 2.12 plot the residuals of the estimated cost weights and actual observations 33 The plots do not reveal an obvious time-related pattern, indicating that the models explain the behaviour of cost weight across time.

One feature in the residual plots is that there appears to be less variation in the residuals following the reforms. This suggests that the reforms may have generated more uniform patterns of patient treatment. In Chapter 4, we consider whether a poorly informed administrator may authorise more consistent, but less appropriate, treatment.

In a final step, as noted in the method in section 2.4, we examined the impact of using the linear form of the cost weight variable. Using this form in OLS regression can result in negative cost weight predictions, which are unrealistic. Models 6 and 7 are identical to models 4 and 5, except that they use the log of cost weight as the dependent variable. The log of cost weight ensures a positive cost weight is predicted. Taking the anti-log of the predicted values lets us make comparisons with the estimates from the linear dependent variable models 4 and 5 .

\footnotetext{
${ }^{33}$ See footnote 29 on page 37 for a discussion of residuals and their usefulness.
} 


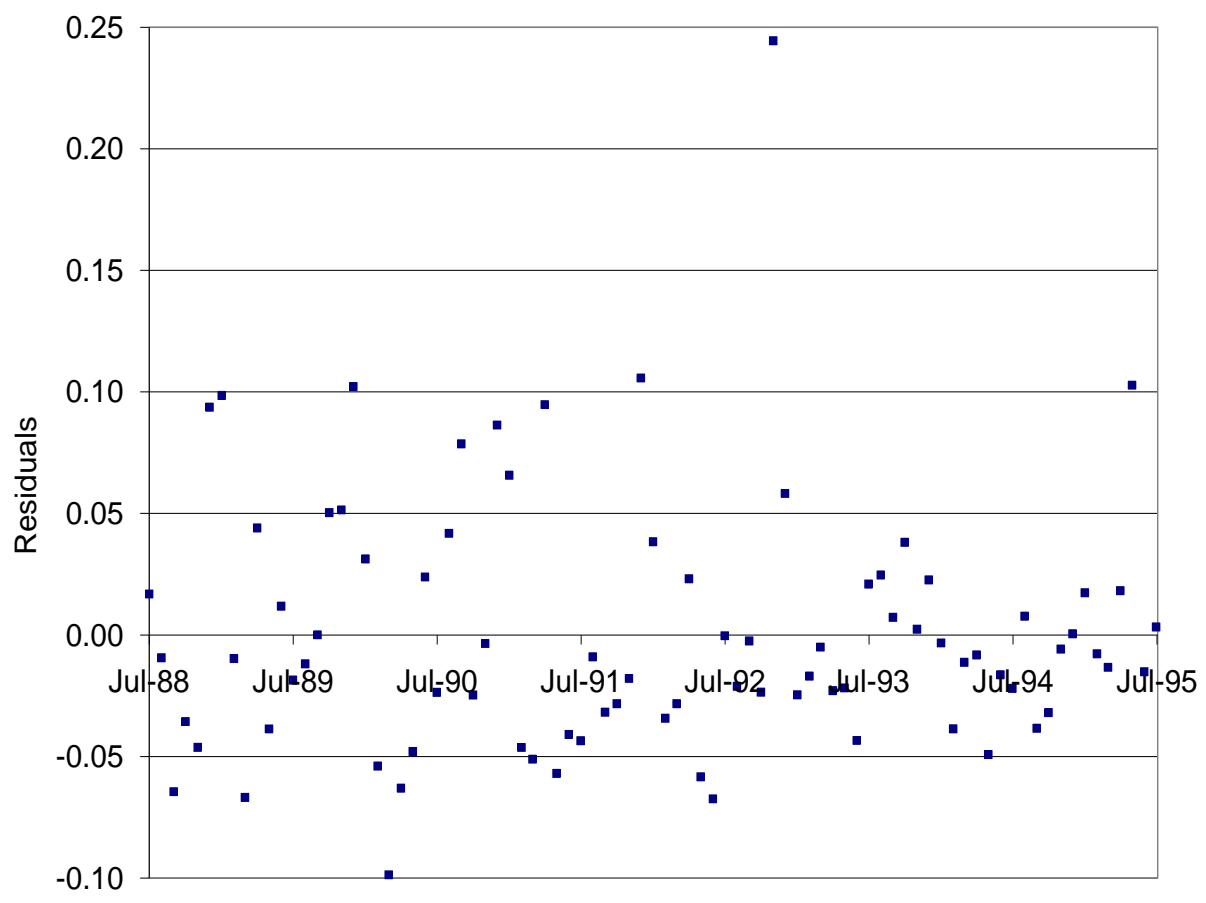

Figure 2.11: Residual plot of Model 4 $C W=\alpha_{0}+\beta_{0}$ period $+\alpha_{1}$ age $+\beta_{1}$ period $*$ age $+\alpha_{2}$ age ${ }^{2}+\beta_{2}$ period $*$ age $e^{2}+\alpha_{3}$ month $+\beta_{3}$ period $*$ month

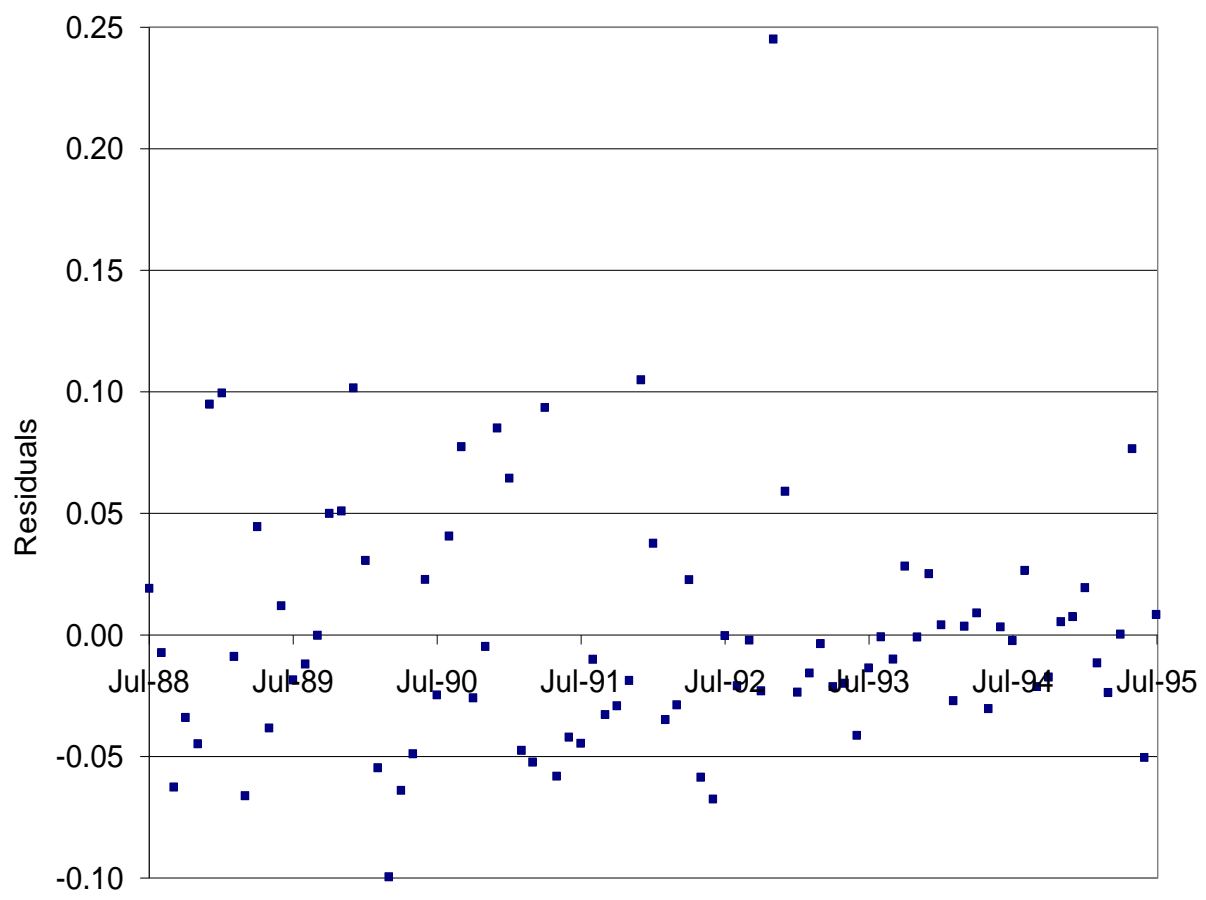

Figure 2.12: Residual plot of Model 5

\footnotetext{
$C W=\alpha_{0}+\beta_{0}$ period $+\alpha_{1}$ age $+\beta_{1}$ period $*$ age $+\alpha_{2}$ age ${ }^{2}+\beta_{2}$ period $*$ age $e^{2}+\alpha_{3}$ month $+\beta_{3}$ period $*$ month $+\alpha_{4}$ month ${ }^{2}+\beta_{4}$ period $*$ month $^{2}$
} 
This analysis gave similar relationships to the linear dependent variable models. Both models 6 and 7 showed that costs would have been higher than in the reforms' absence. This supplementary analysis suggests the results are robust to the form of the dependent variable, and that the linear form is sensible.

\subsection{Discussion}

The empirical analysis supports the claim that the reforms were independently associated with an increase in costs. Both the linear and quadratic time trend models predict a higher cost weight at any point following the reforms after controlling for a background time trend and changes in the age distribution.

In the regression models, the change in treatment costs due to the reforms is measured by the joint effects on the intercept and trend. This change is sensitive to the structure of the model and whether a transitional period immediately following the reforms is censored.

Including a linear trend and period interaction variables, as in Model 4, allowed a simple examination of whether the reforms were associated with a change in the age relationship and background time trend in costs. This model predicted a statistically significant increase in costs immediately after the reforms. Section 2.5.2 considered the possibility that it may have taken some time for the full effects of the reforms to manifest after the CHEs' formal establishment date, which would confound detecting a change in costs using the period variable. Supplementary analyses that censored data in the three month period six month period following the reforms both gave significant increases after the reforms for both the linear and quadratic time trend models.

A more complex, but more appropriate, quadratic trend model allows costs to fall without becoming negative. Model 5 uses a quadratic time trend, and its results also support our hypothesis. This model produces a statistically significant net increase in costs immediately after the reforms, controlling for independent age and background time trend effects. The predicted cost weight rose by 14.5 percent in the month following the reforms. 
The quadratic model indicates some temporary improvements in aspects of cost control immediately after the reforms, with a steepening of the downward trend in cost weight following the reforms. However, within the next year, the slope of the predicted cost weight function flattens then becomes positive due to the reforms.

The analysis of different model structures shows that the relationship between the reforms and treatment costs is likely to have been complex. We estimate that the reforms increased treatment costs, but the magnitude depends on the model structure imposed. This analysis supports our hypothesis that the reforms had an undesirable effect on cost control. This might be interpreted as a reflection of worsening relationships between hospital staff, which led to cost increases immediately after the reforms and then on-going weaker cost control.

The results from the empirical analysis motivate the theoretical models in the following chapters. In Chapter 3 we consider how a funder might incentivise an administrator with a budget target and where a budget blowout puts the administrator's performance incentive at risk. Chapter 4 then investigates how stronger managerial incentives for cost control could inadvertently reduce communication and cause cost increases. Chapter 5 examines how the reforms may have given administrators incentives that could reinforce this process by permitting administrators to limit the flow of information from medical specialists to the public. 


\section{Chapter 3}

\section{Designing delegation incentives in an uncertain environment}

\subsection{Introduction}

The first criticism of the CHE reforms outlined in Chapter 1 was that CHE Boards did not give managers strong incentives, as managers received bonuses despite failing to achieve initial performance targets (Ashton 1999, 142). These targets included operating within a specified budget. Instead, the hospitals' ultimate budgets were increased following cost over-runs, which is consistent with the findings in Chapter 2, In this chapter, we show that paying bonuses in organisations suffering cost over-runs may be efficient because it incentivises additional effort when it is most required.

Budget blow-outs might be understood with reference to the literature on soft budget constraints (SBCs). In this literature, the revised budgets would be viewed as a failure by funders to commit to a hard budget constraint (HBC), resulting in ex ante inefficiency. However, conventional SBC models neglect three important features of the CHE reforms: funder bargaining power, contracts with revisable targets, and the use of performance bonuses. We introduce these features formally in an alternative model to the conventional SBC approach. We generate a novel result that paying bonuses, despite apparent performance failures, can be a feature of efficient contracting. 
We address the following research question:

If a funder is uncertain about an organisation's operating environment, might budget revision and a bonus encourage an administrator to provide an efficient level of effort ex ante, while allowing ex post cost efficiency?

With an HBC, a funder is able to commit credibly to an initial budget. Such a commitment can strengthen an agent's ex ante incentives to work towards meeting the constraint 11 However, we argue that an $\mathrm{HBC}$, and the incentives it creates, is not always in the best interests of the principal. We examine formally how to incentivise an administrator appropriately when an HBC is undesirable or impractical.

We show that where there is uncertainty, a funder may do better by retaining contracting flexibility than by committing to an HBC. The funder may use budget and bonus flexibility to enhance, rather than to undermine, the administrator's incentives. Therefore, budget revisions and bonus payments may be an appropriate response to new information available to the funder, not a failure of managerial efficiency.

The chapter continues as follows: section 3.2 provides background to the CHE reforms, and how the reforms sought to delegate authority more effectively. Section 3.3 reviews two relevant sets of literature: delegation motives and SBC problems.2 Section 3.4 develops a flexible budget constraint (FBC) model as an alternative to the standard SBC approach, as the standard approach sees budget revision as a sign of organisational inefficiency. We examine the conditions under which an $\mathrm{FBC}$ regime may be preferable to an $\mathrm{HBC}$ one.

\subsection{Delegation and performance incentives in CHEs}

The corporatisation of hospitals saw the Minister of Health delegate governance authority to the Boards of Directors of the CHEs. Each Board appointed a single CEO, who

\footnotetext{
${ }^{1} \mathrm{An} \mathrm{HBC}$ is one that a principal commits to not revising even if the organisation were to make a loss and potentially be liquidated.

${ }^{2}$ Appendix A.2.1 presents a simple model of delegation as a motivation for the model in this chapter. This simple model shows that a funder can improve cost efficiency by delegating authority to an administrator even where the administrator's incentives may not be aligned with those of the funder.
} 
replaced a hospital's "triumvirate" management team. CHE CEOs had a mandate for organisational change (Upton 1991), to ration services better within capped funding (Crown Company Monitoring Advisory Unit 1996, 8).

In addition to the radical changes to the management structure, the reformers also changed hospital managers' incentives. CHE CEOs had service and budget targets, and substantial performance bonuses related to controlling costs through efficiency gains. The reformers controlled the top of the organisation and used their power to appoint a high proportion of managers without health sector experience. This suggests that the reformers, not managers, had substantial bargaining power.

Few CHEs successfully controlled their budgets during the reforms, and the CHEs commonly ran substantial deficits. Their CEOs received bonuses despite their apparent failure to rein in deficits. The next section considers the literature on the effectiveness of delegation and how budget constraints may shape managers' incentives to meet those constraints and pursue efficiency.

\subsection{Literature}

Two strands of literature provide a base that the model developed in this chapter extends. The first strand focuses on authority in organisations and the motivations for delegating authority. The second strand is on the SBC syndrome: environmental factors that make it difficult to incentivise a manager.

\subsubsection{Delegation and authority in organisational hierarchies}

We begin by reviewing the first strand of literature on generic motivations for delegation, its extension by Aghion \& Tirole (1997), and Vaithianathan's (1999b) application of agency theory to New Zealand's health sector reforms.

In general, a principal may delegate to make use of an agent's greater inherent skill or their ability to extract information (Alchian \& Demsetz 1972, Holmström 1982). Dele- 
gation gives an agent different incentives than a market-based contract or direct control (Williamson 1985, Aghion \& Tirole 1997). This can encourage participation and incentivise the agent to gather information and invest in effort, which yields benefits to the principal (Aghion \& Tirole 1997, 3). In a political context, delegation can allow politicians to commit to a process, such as health care prioritisation, while insulating themselves from decisions the process entails, such as denying some people care.

Delegation in itself, however, does not necessarily confer the power to make efficient decisions (Tirole 1986, Aghion \& Tirole 1997). In the context of New Zealand's public hospital system, it is likely that the pre-reform triumvirate management teams had real authority and could make good decisions. The management team included medical staff, and the management team's interests were seen as being aligned to those of the clinical staff (Arthur Anderson and Co 1987a). Delegation to a CEO might have conferred formal authority, but it did not necessarily confer real authority.

The reformers expected that corporatisation of hospitals and the new system of delegation would replace "poor management" with "good management", as managers would gather "accurate and relevant information and [have] appropriate incentives to use it" (Hospital and Related Services Taskforce 1988, 26). This quote highlights how the reformers expected CHEs to deliver managers better information (Arthur Anderson and Co $1987 a$, Hospital and Related Services Taskforce 1988).

Vaithianathan $(1999 a, 175)$ concludes that a problem facing a CHE manager was to secure "sufficient real authority within the hospital to enable him to make efficiency-improving changes", while the impediments were "the doctor's strong power base" and "the alternative lucrative employment opportunities that doctors enjoy". As such, delegating authority to a single hospital CEO with strong incentives for service prioritisation might not have conferred real authority, or have passed on these incentives to actors in the hierarchy.

The organisational environment can pose problems for incentivising managers appropriately, as well as the structure for delegating real authority. The SBC literature offers a 
second point of departure to investigate the incentives created in the CHE reforms, and CHE performance.

\subsubsection{The soft budget constraint syndrome}

The SBC literature addresses situations in which a funder persistently supports a lossmaking enterprise. It considers the incentive effects on managers operating in an environment where a funder is unwilling to sanction an under-performing organisation by stopping its funding. Kornai (1998) has referred to this outcome as the "SBC syndrome", to capture the wide set of contexts and factors that may be associated with this outcome. For example, the SBC syndrome may occur in socialist or capitalist systems, and intervention may be ex ante subsidies or ex post bail outs (Dewatripont \& Maskin 1995, Bai \& Wang 1998, Kornai 1998).

The conventional SBC framework allows actors to have a range of objectives, and can be applied to a wide range of organisation types. Kornai $(1998,15)$ characterises SBC theory as a theory of exit, where "an SBC perspective does not require that economic agents are profit maximizers. Rather it is sufficient to assume that the decision maker is intent on survival and avoiding organisational death". This flexibility is useful in considering how managers are incentivised in organisations where it may be politically unpalatable to shut such organisations down ${ }^{3}$ In this case, a manager may focus less on income and profit, and more on securing the organisation's on-going existence despite making persistent losses (Kornai 1998, 15). The flexibility of this framework is useful for thinking about CHEs, which were state owned companies instilled with private sector disciplines, but where the closure of larger hospitals would have been politically difficult.

The SBC literature has provided a variety of explanations as to why a manager may have weak incentives to meet a funder's budget target (Kornai 1980, Kornai 1986, Schmidt 1996,

\footnotetext{
${ }^{3}$ Although the issue of shutdown is a key one in public sector theory, SBC theory also applies to private sector institutions. Kornai $(1998,12)$ argues that the SBC syndrome is more likely with state ownership than in the private sector as "state ownership provides the government with much stronger inducements to soften the budget constraint".
} 
Kornai 1998, Bai 1998, Bai, Li, Tao \& Wang 2000, Maskin \& Xu 2001, Kornai 2001). In this literature, a budget increase represents a funder's ex post efficient response to actual conditions, but ex ante this response is inefficient. However, these models make assumptions that do not sufficiently mirror the features of New Zealand's CHE reforms.

Another feature of the SBC syndrome is that it occurs where there are vertical relationships, which involve the authority of a superior over a subordinate (Kornai 1998, 12).4 This is relevant to the stylised CHE organisational hierarchy being developed through this thesis.

It is commonly argued in the literature that SBC problems are worse for public organisations than for private ones. This result may reflect the reluctance of public sector organisations to ignore negative externalities, or the different objectives followed by public and private organisations.

Schmidt $(1996,1)$ contributes to the SBC literature by arguing that privatisation can reduce the information the government can access, which can commit it to an HBC. The choice of governance structure in this environment creates a tradeoff between allocative and productive efficiency in some circumstances: production levels may be lower than efficient but a manager has better incentives to save costs. While Schmidt (1996) allows the principal to have full bargaining power, a feature of the CHE reforms incorporated in our model, his approach and focus are not well suited to the CHE reforms. The reforms aimed to improve information available to managers and the government's monitoring agencies, not to reduce it as in Schmidt's (1996) model.

The organisational structure and environment of CHEs differed in a number of respects from those envisaged by the conventional SBC literature. As such, this literature offers limited insight into the impact of the reforms on CHE performance. First, the CHE reformers arguably had strong bargaining power over managers. Second, the CHE managers were intentionally drawn from a common pool of labour that served the public and

\footnotetext{
${ }^{4}$ In a horizontal relationship, two parties negotiate but neither has authority over the other, although the levels of bargaining power may differ.
} 
private sectors, and the managers were given contracts with strong at-risk performance incentives. Third, despite CHE operational independence and explicit service contracts, hospital deficits could still be funded ex post if expenditure turned out to be higher than the contracted service volumes (Vaithianathan 1999b, 145-46). Fourth, corporatisation was expected to improve management information systems that were weak before the reforms. Although this information was often controlled by clinical staff (Arthur Anderson and Co $1987 a$, Arthur Anderson and Co 1987b), the organisation's overall costs were observable and contractible. The choice of governance structure was unlikely to have aimed to reduce the production and transfer of precise information to the government. As we show in Chapter 4, however, incentives on managers may have unintentionally encouraged managers to limit communication of information.

\subsection{Budget revision and bonuses: an alternative to conventional SBC models}

In this section we outline how a funder might use an initial budget and bonus offer to encourage an administrator to pursue cost efficiency $!^{5}$ We modify the conventional SBC model to include three stylised features of the CHE reforms: funder bargaining power, contracts with revisable targets, and the use of performance bonuses.6 Given our modelling innovations and novel results, we refer to our model as an FBC model to distinguish it from a conventional SBC model.

First, we assume that the principal has all the bargaining power, rather than being 'held up' in ex post renegotiations with the agent. Second, we assume that some variables are verifiable at an intermediate step in the game. This allows the funder to write an initial contract, and revise it at a later step. Third, our model explicitly includes performance bonuses to connect the administrator's effort to the organisation's performance. Our

\footnotetext{
${ }^{5}$ For ease of reading, we assign the actors genders. These genders are used consistently throughout the thesis. In this chapter we introduce a funder (male) and an administrator (female).

${ }^{6}$ Appendix A.2.2 outlines two models that bridge between the SBC literature and this chapter's main model. The first shows a conventional SBC result, and the second incorporates bonus payments. Neither of these simple models, however, reflects the stylised facts of the CHE reforms examined here.
} 
model generates a novel result that paying bonuses, despite apparent performance failures, can be a feature of efficient contracting.

\subsubsection{Play of the game}

This is a one-period game and has two versions: an HBC regime and an FBC regime. Its steps are as follows:

1. The funder, $F$, offers an administrator, $A$, a contract with a budget target, $b_{1}$, and a remuneration package. $\left(w_{1 M}, w_{1 N}\right) \in \mathbb{R}_{+}^{2} ; A$ is entitled to a bonus of $w_{1 M}$ if she meets the target and $w_{1 N}$ otherwise.

2. $A$ observes the offer and decides whether to take the offer, $O$. If she rejects it, $Q$, then the players receive their reservation payoffs, $\bar{u}_{F}$ and $\bar{u}_{A}$. Otherwise ...

3. A chooses to put in high or low effort to an initial task, $e_{1} \in\{L, H\}$.

4. $N$ determines the organisation's baseline costs, $c \sim G_{e_{1}}$.

5. $F$ observes $c$ and $e_{1}$. Under an HBC regime the contract in the second stage has the same budget target, $b_{2}=b_{1}$, and no bonus revision, $w_{2}=0$. Under an FBC regime, $F$ can offer a revised contract.7

6. A chooses to put in high or low effort to a second task, $e_{2} \in\{L, H\}$. This influences the organisation's ultimate costs, $\kappa$.

Figure 3.1 on page 57 illustrates the steps of the game. The players' utility functions are given in equations 3.1 and 3.2 on page 57 .

Budgets do not directly affect the organisation's ultimate costs. That is, the funder sets a budget target, $b$, but ultimately incurs the actual treatment cost $\kappa$. This implies that any budget surplus would be fully recovered, while budget deficits are fully funded.

\footnotetext{
${ }^{7}$ If $A$ rejects the revised contract, the original contract is binding.
} 


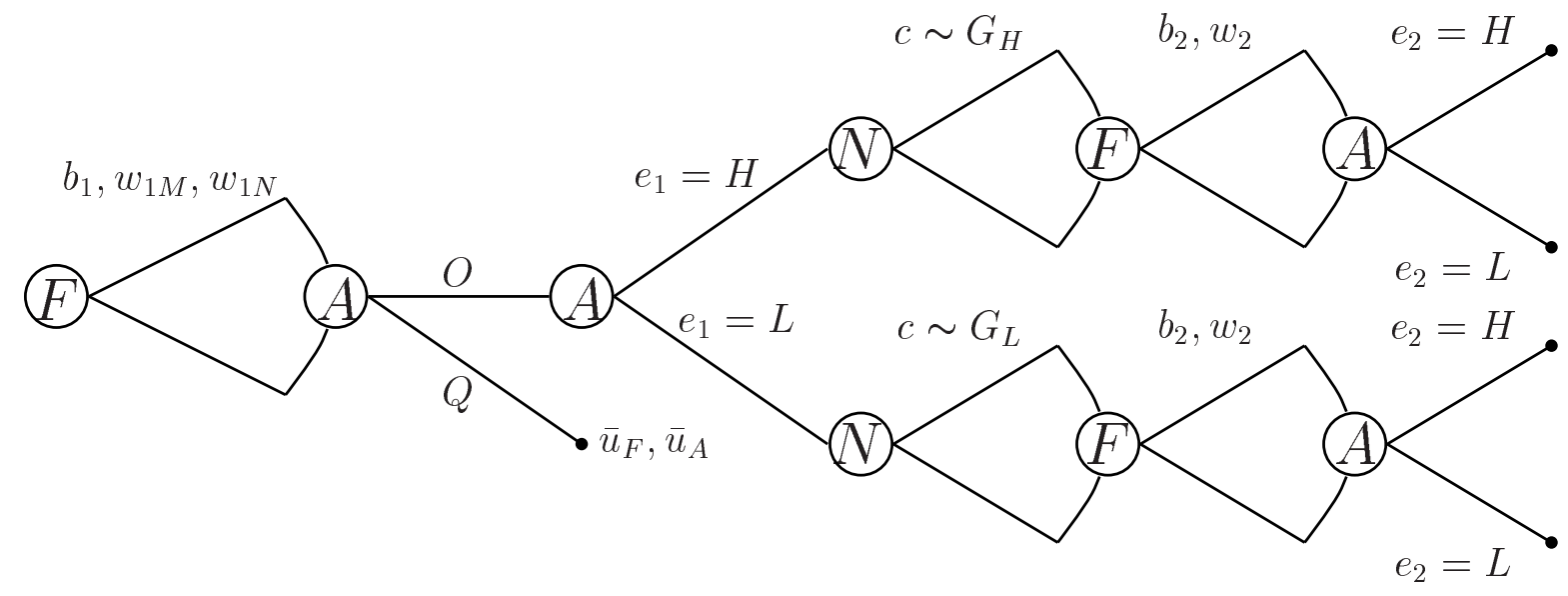

Figure 3.1: Game tree for budget-bonus model

The funder seeks to minimise expected total costs and is risk-neutral; the administrator is risk-averse ${ }^{89}$ The players' utility functions are given below; $\kappa$ and $\omega$, the administrator's bonus payment, are defined below on pages 57 and 59 .

$$
\begin{aligned}
& U_{F}=-[\omega+\kappa] \\
& U_{A}=v(\omega)-\left(e_{1}+e_{2}\right) .
\end{aligned}
$$

The organisation's ultimate costs depend on both Nature's choice of $c$ and the effort the administrator puts into the second task:

$$
\kappa= \begin{cases}c & \text { if } e_{2}=L \\ c-\delta & \text { if } e_{2}=H\end{cases}
$$

Nature's choice of the organisation's baseline costs, $c$, can be thought of as reflecting the environment, for example, whether the people presenting for treatment tend to be simple or complex cases.

\footnotetext{
${ }^{8}$ This implies that for the administrator, $0<v^{\prime}(\omega)$ and $v^{\prime \prime}(\omega)<0$.

${ }^{9}$ In the context of New Zealand's extensive public secondary health service, the widespread closure of public hospitals that ran deficits, and consequent service shortage, would likely have been less politically palatable than deficits. This motivates the funder's cost minimisation objective.
} 
We assume that:

1. the administrator's first task determines the cost distribution, $c \sim G_{e_{1}}$.

2. the distributions $G_{L}$ and $G_{H}$ have the same support $[0, \bar{c}]$, where $\bar{c}$ is an upper cost limit.

3. $G_{L}$ exhibits first order stochastic dominance over $G_{H}{ }^{10}$ That is, the probability of meeting the budget target with high effort in the initial task is greater than with low effort for any $b_{1}$ that the funder might set.

4. high effort in the second task reduces the organisation's baseline costs by $\delta$. If the administrator were to provide high effort in the second task, ex ante her effort increases the cumulative probability of satisfying the original budget target.

5. low effort in either period has zero disutility, $L=0$, and high effort in either period has the same disutility, $H$.

Budgets and costs are contractible. This means the funder can make the initial bonus offer, $w_{1 i}$, contingent on whether or not costs in the initial stage exceed the baseline budget, $b_{1}$, not just on whether costs exceed the ultimate budget, $b_{2}$.

The administrator's effort is non-contractible and she receives a single bonus payment at the end of the game, $\omega$. The difference between $w_{1 M}$ and $w_{1 N}$ can be thought of as a performance-related component of the administrator's remuneration package.

Under the $\mathrm{HBC}$ regime, the administrator receives a bonus, $\omega=w_{1 M}$, if she achieves the initial budget target and $\omega=w_{1 N}$ if she does not; we assume $w_{2}=0$ under this regime. Under the FBC the administrator receives $\omega$ as described in array (3.5). For example, under the FBC regime, if the administrator achieves the budget targets under both the original and revised contract, the bonus payment is $\omega=w_{1 M}+w_{2}$.

\footnotetext{
${ }^{10} G_{L} \leq G_{H}$ for all $c \in[0, \bar{c}]$ and $G_{L}<G_{H}$ for some $c$.
} 
In the HBC regime, $\omega= \begin{cases}w_{1 N} & \text { if } b_{1}<c \\ w_{1 M} & \text { if } b_{1} \geq c\end{cases}$

In the FBC regime, $\omega= \begin{cases}w_{1 N} & \text { if } b_{1}<c \text { and } b_{2}<\kappa \\ w_{1 N}+w_{2} & \text { if } b_{1}<c \text { and } b_{2} \geq \kappa \\ w_{1 M} & \text { if } b_{1} \geq c \text { and } b_{2}<\kappa \\ w_{1 M}+w_{2} & \text { if } b_{1} \geq c \text { and } b_{2} \geq \kappa\end{cases}$

Under an FBC, the original contract affects the administrator's bargaining position when it comes to revising her contract. For example, if $c \leq b_{1}$ at step 5 , then the administrator could meet the budget target with $e_{2}=L$ and receive a payoff of $v\left(w_{1 M}\right)-e_{1}$. Incentivising high effort in the second task would be more costly to the funder than if the administrator was not already eligible for a bonus. The funder would need to allow for this in designing the original contract, and in considering a contract revision at step 5 in the $\mathrm{FBC}$ regime. Given our interest in the CHE reforms, which incentivised managerial effort, we assume that high effort in the second task is efficient. That is, we assume the funder would be willing to pay for such effort. Ex post, the maximum the funder would be willing to pay for high effort in the second task is $\delta$. As the administrator is risk-averse, it is plausible that the administrator would be willing to accept less money to provide high effort than if she were risk-neutral. This idea is captured in the following assumption, where $x$ represents a generic measure of income.

$$
v(x)<v(x+\delta)-H \quad \forall x \in \mathbb{R}_{+}
$$

The funder's willingness to pay for high effort in the second task, $\delta$, is higher than the administrator's willingness to accept, in equation (3.6). Thus, the funder and administrator should be willing and able to negotiate a bonus that results in high effort in the second task. 
We use backward induction to solve this game for a specific set of assumptions about the effect of effort on costs. We focus on a case where the funder desires high effort for both tasks 11 This case is consistent with the desire of CHE reformers that CEOs work to redesign the systems and incentives throughout a hospital to deliver efficiency gains.

\subsubsection{A hard budget constraint regime}

In this section we examine the funder's choices under an $\mathrm{HBC}$ regime, which provides a benchmark for comparison under an FBC ${ }^{12}$ We begin by considering the contract design problem for effort in the second task, and then consider the design problem for effort in the initial task.

\section{The second stage}

At step 5 in Figure 3.1, both players have observed the organisation's baseline costs, resolving any uncertainty. Below, we consider three cases distinguished by whether the administrator needs to provide high effort in the second task in order to meet the original budget target.

\section{- Case 1: high effort not required under the original budget}

When $c \leq b_{1}$, the administrator does not need to provide high effort in the second task in order to meet the initial budget target and to be eligible for $w_{1 M}$. With an HBC, at this stage of the game, the funder is unable to incentivise the administrator to put high effort into the second task. In this case, $\kappa=c$ and the administrator's payoff is:

$$
v\left(w_{1 M}\right)
$$

\section{- Case 2: high effort required under the original budget}

Now consider a case with higher costs: when $c-\delta \leq b_{1}<c$, the administrator can only

\footnotetext{
${ }^{11}$ More generally, the equilibrium contract will depend on the assumptions about the effects of effort on costs. These assumptions influence the funder's preferred level and mix of effort in equilibrium.

${ }^{12}$ The closure of larger public hospitals would have been politically difficult in New Zealand. Therefore, imposing an HBC, and committing to shut down under-performing hospitals, would not have been practical. Footnote 3 on page 53 discusses the treatment of the shutdown decision in the SBC literature.
} 
satisfy the original budget constraint by exerting high effort in the second task. For the administrator to wish to exert this effort, the following condition must hold:

$$
v\left(w_{1 N}\right) \leq v\left(w_{1 M}\right)-H
$$

In this case, the original budget constraint and bonus, $w_{1 M}$, would induce the administrator to provide high effort in the second task. In this case, $\kappa=c-\delta$ and the administrator's payoff is:

$$
v\left(w_{1 M}\right)-H
$$

\section{- Case 3: budget violation even with high effort}

In the third case, costs are higher again, $b_{1}<c-\delta$. As the organisation's baseline costs are higher than the initial budget, the administrator is unable to meet the original budget target even with high effort. She would not provide high effort in the second task under the original contract as she would only receive a base payment of $w_{1 N}$. In this case, $\kappa=c$ and the administrator's payoff is:

$$
v\left(w_{1 N}\right)
$$

Thus, the administrator provides high effort in the second stage in only one of the three cases under an HBC regime. The bonus induces high effort in case 2 only.

\section{The initial offer}

The problem facing the funder at step 1 is to design a contract that induces the level of effort in the initial task that minimises total expected costs, given the incentives required for high effort given in (3.7), (3.8) and (3.9) above.

We denote the ex ante cumulative probability of achieving the initial budget constraint given some level of effort in the first task, but without high effort in the second task, by $G_{e_{1}}\left(b_{1}\right)$. High second stage effort lowers the ultimate cost by $\delta$ and is equivalent to 
increasing the budget to $b_{1}+\delta$. We denote the ex ante cumulative probability with high effort in the administrator's second task by $G_{e_{1}}^{\delta}\left(b_{1}\right){ }^{13}$

The funder's objective is to choose $b_{1}, w_{1 M}$, and $w_{1 N}$ in order to minimise the following, subject to satisfying the administrator's participation constraint and incentive compatibility constraint for high effort in the initial task:

$$
G_{H}\left(b_{1}\right) w_{1 M}+\left[G_{H}\left(b_{1}+\delta\right)-G_{H}\left(b_{1}\right)\right]\left(w_{1 M}-\delta\right)+\left[1-G_{H}\left(b_{1}+\delta\right)\right] w_{1 N}+E_{H}[c] .
$$

Figure 3.2 is an illustrative example of a cumulative probability distribution of costs depending on the administrator's effort in the first task. $G_{H}$ is the distribution induced by high effort, and $G_{L}$ by low effort. The figure shows the ex ante cumulative probability of achieving a particular budget target with or without high effort in the administrator's second task.

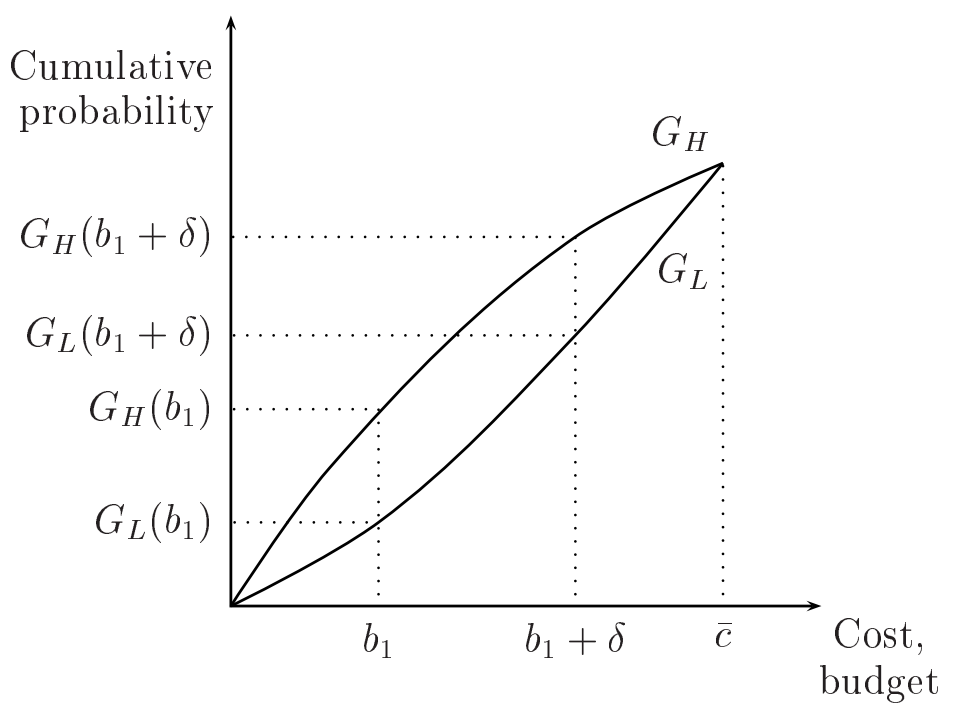

Figure 3.2: Cost function examples

The initial bonuses must satisfy two constraints to induce the administrator to provide high effort in the initial task. The participation constraint requires that the administrator receives an expected utility that is at least as great as her reservation utility, $\bar{u}_{A}$. Thus,

${ }^{13}$ We abbreviate the terminology $G_{e_{1}}\left(b_{1}\right)$ to $G_{e_{1}}$ and $G_{e_{1}}\left(b_{1}+\delta\right)$ to $G_{e_{1}}^{\delta}$. 
the participation constraint can be expressed as follows:

$$
\bar{u}_{A} \leq G_{H} v\left(w_{1 M}\right)+\left[G_{H}^{\delta}-G_{H}\right]\left(v\left(w_{1 M}\right)-H\right)+\left[1-G_{H}^{\delta}\right] v\left(w_{1 N}\right)-H .
$$

The three elements in the participation constraint in equation 3.12 reflect the payoffs that the administrator expects to receive in the second stage as set out in equations (3.7), (3.8) and (3.9). The funder's bargaining power lets him hold the administrator to her participation constraint. This requires the administrator be indifferent between participating (and providing effort) or not.

The second constraint is an incentive compatibility constraint. This constraint requires that the expected bonus be sufficient to encourage the administrator to provide high effort in the first task:

$$
\begin{aligned}
& G_{L} v\left(w_{1 M}\right)+\left[G_{L}^{\delta}-G_{L}\right]\left(v\left(w_{1 M}\right)-H\right)+\left[1-G_{L}^{\delta}\right] v\left(w_{1 N}\right) \\
\leq & G_{H} v\left(w_{1 M}\right)+\left[G_{H}^{\delta}-G_{H}\right]\left(v\left(w_{1 M}\right)-H\right)+\left[1-G_{H}^{\delta}\right] v\left(w_{1 N}\right)-H .
\end{aligned}
$$

The choice of $b_{1}$ will depend on the distributions of $G_{L}$ and $G_{H}$. But to incentivise high effort in the initial task, the funder must set the initial budget below the upper cost limit, $\bar{c}$. If the funder sets too relaxed a budget target, for example $\bar{c} \leq b_{1}$, then the administrator would achieve the target whatever her level of effort. The incentive compatibility constraint would become $v\left(w_{1 M}\right) \leq v\left(w_{1 M}\right)-H$, which can never be satisfied. Thus, to implement high effort in the first task, the funder sets the initial budget below the maximum cost, $b_{1}<\bar{c}$.

For any given choice of $b_{1}$, the funder's choice of $w_{1 M}$ and $w_{1 N}$ follows a standard moral hazard model with discrete effort levels, a risk averse agent and a risk neutral principal ${ }^{14}$ In such a model, the principal minimises the risk that the agent bears, while incentivising the principal's preferred action, for example high effort. The optimal incentive scheme will involve the agent bearing some risk, and the optimal solution occurs at the intersection of two constraints: the agent's participation and incentive compatibility constraints.

\footnotetext{
${ }^{14}$ See for example, Chapter 25 of Varian (1992).
} 
Recall that in our model, the funder is risk-neutral and the administrator risk-averse.

The funder aims to choose a contract that gives as little risk to the administrator as possible, without undermining her incentive for high effort in the first task. If the funder can implement high effort in the first task using an HBC, then the constraints in equation (3.12) and equation 3.13 hold with equality.

Solving the constraints given by equations 3.12 and (3.13) simultaneously yields the following solutions. We use the following notation below: $\Delta_{H}=G_{H}^{\delta}-G_{H}$ and $\Delta_{L}=$ $G_{L}^{\delta}-G_{L}$

$$
\begin{aligned}
& v\left(w_{1 N}\right)=\bar{u}_{A}-\frac{G_{L}^{\delta}}{G_{H}^{\delta}-G_{L}^{\delta}} H+\left[\Delta_{H}-\frac{G_{H}^{\delta}}{G_{H}^{\delta}-G_{L}^{\delta}}\left(\Delta_{H}-\Delta_{L}\right)\right] H \\
& v\left(w_{1 M}\right)=\bar{u}_{A}+\frac{1-G_{L}^{\delta}}{G_{H}^{\delta}-G_{L}^{\delta}} H+\left[\Delta_{H}+\frac{1-G_{H}^{\delta}}{G_{H}^{\delta}-G_{L}^{\delta}}\left(\Delta_{H}-\Delta_{L}\right)\right] H
\end{aligned}
$$

These solutions implicitly define the funder's choices of $w_{1 M}$ and $w_{1 N}$ when the funder wants high effort in both of the tasks. These solutions may be used in equation (3.11) to determine $b_{1}$ implicitly.

\subsubsection{A flexible budget constraint regime}

This section shows that the funder can benefit from an FBC regime relative to the HBC regime described above.

\section{The revised offer}

At step 5 in Figure 3.1, if the funder has set the initial budget below the upper cost limit,

$\bar{c}$, then he may sometimes wish to revise the budget at this step, paired with a revised bonus, to incentivise the desired level of effort in the second stage.

The original contract, along with the organisation's baseline costs $c$, determines the minimum bonus that would induce the administrator to put high effort into the second task, 
$e_{2}=H$. A revised bonus must satisfy the following constraint for the administrator to have an incentive to provide high effort. We refer to $w_{1 i}, i \in\{N, M\}$, so we can express the constraint in a generic form, but the players know whether the administrator has met the original target and is eligible for $w_{1 M}$ or $w_{1 N}$ :

$$
v\left(w_{1 i}\right) \leq v\left(w_{1 i}+w_{2}\right)-H \quad i \in\{N, M\}
$$

It is convenient to have a shorthand expression for the revised bonus the funder wishes to offer. Let $\mathfrak{w}\left(w_{1 i}\right)$ represent the minimum revised bonus that is compatible with high effort, given that the administrator could earn $w_{1 i}$ with low effort, which has zero disutility to the administrator (by assumption (5) on page 58):

$$
\mathfrak{w}\left(w_{1 i}\right)=\min \left\{w_{2} \mid v\left(w_{1 i}\right) \leq v\left(w_{1 i}+w_{2}\right)-H\right\}
$$

Below, we consider the three cases of whether high effort is required to meet the original budget target.

\section{- Case 1: high effort not required under the original budget}

Under an FBC regime, when $c \leq b_{1}$, the funder would offer the minimum revised bonus that would induce the administrator to put high effort into the second task, given that the administrator is eligible for $w_{1 M}$ even with $e_{2}=L$. That is:

$$
w_{2}=\mathfrak{w}\left(w_{1 M}\right)
$$

The funder would need to pair the revised bonus with a revised budget target, otherwise the administrator would be able to earn $w_{1 M}+w_{2}$ with low effort. The funder should tighten the budget target so that the revised bonus is contingent on high effort in the second task, $c-\delta \leq b_{2}<c$.

The funder's bargaining power lets him hold the administrator to her incentive compatibility constraint, so the administrator's payoff in this case is:

$$
v\left(w_{1 M}\right)=v\left(w_{1 M}+w_{2}\right)-H
$$




\section{- Case 2: high effort required under the original budget}

As in the HBC regime, when costs are higher, $c-\delta \leq b_{1}<c$, the administrator can only satisfy the original budget constraint by exerting high effort in the second task. For the administrator to wish to exert this effort, the following condition must hold:

$$
v\left(w_{1 N}\right) \leq v\left(w_{1 M}\right)-H
$$

As the original budget constraint and bonus, $w_{1 M}$, would induce the administrator to provide high effort in the second task, it is unnecessary to revise the budget and bonus. The funder could offer, for example, $w_{2}=0$ and leave the budget at its original level, $b_{2}=b_{1}$.

In this case, as the contract is not renegotiated, the administrator's payoff is:

$$
v\left(w_{1 M}\right)-H
$$

\section{- Case 3: high effort only with a revised budget}

In the third case, the administrator cannot meet the original budget target even with high effort, $b_{1}<c-\delta$ and lacks the incentive for high effort under the original contract. In this case, the funder would revise the budget to $b_{2}=c-\delta$ and offer an additional bonus that is contingent on the administrator providing high effort in the second task:

$$
w_{2}=\mathfrak{w}\left(w_{1 N}\right)
$$

The administrator's payoff in this case is:

$$
v\left(w_{1 N}\right)=v\left(w_{1 N}+w_{2}\right)-H .
$$

Thus, in an FBC regime, the funder can always secure high effort in the second stage, so $\kappa=c-\delta$ in all three cases.

\section{The initial offer}

Our model is now more complicated than the standard moral hazard model, as there is renegotiation at the start of the second stage. 
We assume that the funder has all the ex post bargaining power. In this case, the funder can renegotiate to secure high effort in the second task and the payoff of the administrator remains the same. Thus, if effort in the first task is implementable under the HBC regime it will also be implementable under an FBC regime. That is, the solutions defined by equations (3.14) and (3.15) are consistent with the conditions for the three cases in the revision stage on pages 65 to 66 .

For example, equation (3.20) requires that $H \leq v\left(w_{1 M}\right)-v\left(w_{1 N}\right)$ for high effort in the second task (in case 2). Equations (3.14) and (3.15) give the following expression:

$$
v\left(w_{1 M}\right)-v\left(w_{1 N}\right)=\frac{H+\left(\Delta_{H}-\Delta_{L}\right) H}{G_{H}^{\delta}-G_{L}^{\delta}} .
$$

Substituting equation 3.24 into equation 3.20 we get:

$$
H \leq \frac{H+\left(\Delta_{H}-\Delta_{L}\right) H}{G_{H}^{\delta}-G_{L}^{\delta}}
$$

This simplifies to:

$$
G_{H}-G_{L} \leq 1
$$

As $G_{L}<G_{H} \leq 1$ by assumption (3) and as they are both probabilities, equation (3.25) is true. Thus, the funder can use the flexible budget and bonus scheme to incentivise the administrator to put high effort into both tasks.

Hence, if the funder can implement high effort in the first task under an HBC regime, he can also implement it under an FBC regime. Furthermore, the funder's payoff will be no lower under an FBC, and will be sometimes be higher. In particular, if high effort in the second task is desirable, then the funder's payoff is higher in cases 1 and 3 above. That is, flexibility is strictly preferable when baseline costs are low (case 1) or high (case 3) as he can offer a revised contract, which reduces costs to $c-\delta$ compared to $c$ under an HBC regime. 


\subsubsection{Outcomes of the game}

Budget targets and bonuses may be used to encourage cost-minimising effort when a funder faces ex ante uncertainty about what total level of effort (and incentive) would be required.

We conclude that the funder may find it efficient to retain contracting flexibility and $e x$ post to pay the administrator a performance bonus even when she fails to meet the original budget target. That is, under high cost conditions the funder may offer a higher revised budget and combine this with a bonus to induce the cost-minimising level of effort.

Furthermore, a contract including a performance bonus conditional on meeting a budget target may minimise expected total cost. Such a contract provides the administrator with appropriate ex ante incentives for high early effort.

We conclude that it can be efficient ex ante to retain budget flexibility, rather than committing to an $\mathrm{HBC}$. If the funder were to commit to an $\mathrm{HBC}$, then in two of the three cases outlined above he would lack the flexibility to adjust the administrator's incentives so that he could incentivise high effort and minimise expected total costs. Under an HBC the funder faces a tension between incentivising high effort in both stages. In particular, tightness in the initial budget makes it more likely that high effort will be required in the second stage, but so that the administrator Thus, an HBC can result in ex post inefficiency, as the funder's commitment prevents him from incentivising the cost-minimising level of effort in the second task.

Our novel conclusion is that budget revision paired with a bonus can encourage an administrator to provide an efficient level of effort ex ante, while allowing ex post cost efficiency. This conclusion contrasts with the results in conventional SBC models. In those models, there is a trade-off between ex ante and ex post efficiency and an HBC may be desirable but is not credible.

In the environment we explore, budget and bonus revisions enable the funder to achieve ex ante and ex post efficiency. We conclude that budget increases associated with bonus 
payments can be efficient, and budget revision is not necessarily a sign of inefficiency. Rather, flexible contracts can be used to give an administrator the correct incentives for efficient early effort, while allowing the funder to tailor the ultimate budget to the actual conditions.

The results above suggest that the CHE reformers may have expected a clear target and a performance bonus to deliver. That is, to provide a new CEO ex ante incentives to redesign the hospital's management systems and encourage efficiency-enhancing changes throughout the hospital. Paying CEOs bonuses despite running unexpected deficits may have helped to elicit extra effort when the reformers realised such effort was needed, that is, it was ex post efficient. Our formalisation can provide an insight into the actions of the CHE funder, which contrasts with the first criticism of the CHE reforms.

\subsection{Discussion}

We show formally that a funder may benefit from contracting flexibility, and an FBC can be preferable to an HBC. Budget and bonus flexibility may enhance, rather undermine, an administrator's ex ante incentives to act in accordance with the funder's objectives. Budgets and bonuses set in an initial stage may give the administrator appropriate $e x$ ante incentives. A tighter ex ante budget demands more effort from the administrator to satisfy the constraint; but it increases the risk of not meeting the target. Ex post revision allows actual conditions to dictate the ultimate efficient budget and bonus levels, while providing the administrator appropriate ex ante incentives for effort.

We find that a delegated organisational structure with budget and bonus flexibility can be an efficient response to an environment with ex ante uncertainty and incomplete contracts. This FBC approach can yield a better result than imposing an HBC. This contrasts with the conventional SBC result where budget revisions represent a failure of managerial efficiency. 
The formal results suggest that the criticism of paying bonuses alongside budget blowouts may not have sufficiently acknowledged the reforms' uncertain environment. Bonus payments may have been a pragmatic way to encourage CEOs to pursue budget targets despite uncertain conditions and rewards. Budget revisions may have been an effective response to unfavourable cost conditions that were beyond the CEO's control to manage. That is, it may have been politically expedient to relax the budget constraints of CHEs rather than to close hospitals that run deficits. This may appear to frustrate the objectives of the reformers. But it may also be seen as the best outcome for a funder given initial uncertainties.

In Chapter 4, we suggest how offering bonuses to control costs could potentially increase total costs. We show that sharp incentives for cost control may result in an environment of uncertainty, less well informed treatment decisions, and higher costs. In the light of this chapter's findings, such a dynamic might cause big budgets. This is contrary to the expectations of the CHE reformers, but is consistent with the claim of poor cost control by CHEs examined in Chapter 2 . 


\section{Chapter 4}

\section{Incentive congruence, credibility and communication}

[T]here was insufficient tension between management and clinical staff

- A CCMAU observation of Christchurch Hospital, Health and Disability Commissioner $(1998,164)$

\subsection{Introduction}

The Crown Company Monitoring Advisory Unit (CCMAU) observation in the quote above reflected a belief amongst some government advisers that consensus management meant hospital managers were not pursuing productivity and cost improvements aggressively enough before the CHE reforms. The reformers introduced strong incentives for CEOs to focus them on the funder's objective of improved prioritisation and cost efficiency ${ }^{1}$

This chapter considers a theoretical basis for a second criticism of the CHE reforms, that although CHE managers were given strong incentives for cost control, many hospitals' costs and deficits escalated. We argue that sharpening an administrator's incentives

\footnotetext{
${ }^{1} \mathrm{CCMAU}$ was responsible for monitoring the performance of public hospitals, as state-owned companies, and providing advice to the shareholding Ministers of Finance and of Health about "maximis[ing] the value from owning these companies". Downloaded 25 August 2004 from http://www.ccmau.govt.nz/aboutUs/whatwedo.asp. CCMAU ceased to be involved in the monitoring of CHEs (or their successors) with the removal of CHEs and a transfer of functions under the Health and Disability Act 2003.
} 
for cost control can impair communication and can result in a worse outcome for the incentivised task.

We develop a theoretical model to answer the following questions:

- If information is not verifiable, can a misalignment between an administrator and a specialist's incentives erode the specialist's credibility with the administrator?

- Can such tension reduce communication between an administrator and a specialist?

- If a funder increases an administrator's incentive to control costs, could the resulting tension between an administrator and a specialist cause a poorer outcome from the funder's perspective?

We add to the literature by modelling the communication of "soft" information as an endogenous choice in a multi-layered organisational hierarchy. We show how sharp incentives may erode an agent's credibility and reduce the meaningful communication of soft information $2^{2}$ Sharper managerial incentives for cost control can cause both higher expected patient harm and higher expected treatment costs. This suggests a tradeoff between sharp incentives that align the objectives of the administrator and the funder, and soft incentives that promote communication between the agents. A novel result of the model is that sharper incentives for a verifiable task can lead to a poorer outcome for that same task.

The empirical analysis in Chapter 2 suggests that patient treatment costs rose after the reformers installed administrators incentivised to control costs. This chapter adds a layer to the organisational hierarchy of Chapter 3. The model in the present chapter separates the role of two actors involved in an organisation's operation, an administrator and a specialist. By dividing these two roles, we can examine how a divergence between the agents' interests might impair the organisation's performance.

\footnotetext{
${ }^{2}$ Our model uses a specialist's recommendation about the appropriate treatment for a patient as an example of the communication of soft information. The model in this chapter may be adapted for more general forms of cooperation, or communication, between agents where credibility is an important dimension to successful cooperation.
} 
This chapter is organised as follows: section 4.2 describes aspects of the CHE reforms to give some context to the second criticism of the CHEs. Section 4.3 reviews the theoretical literature on the undesirable effects of sharp incentives. Section 4.4 presents a model with the novel result that giving managers strong incentives to control costs can increase costs. The chapter finishes with a discussion that links the theoretical model's insights to the empirical analysis in Chapter 2. Appendix A.3.1 provides a proof of the uniqueness, and conditions for existence of the equilibrium outcomes identified in this chapter; and, Appendix A.3.2 provides an illustrative example of the existence of the NPE outcomes.

\subsection{Managers' incentives and the CHE reforms}

CHE Boards of Directors sought "new leadership committed to the new order, rather than old leadership bound by the status quo" (Troughton 1993, 16). As a result, a large proportion of CEOs was appointed from outside the health sector, while CEOs from the health sector were in some cases only appointed provisionally.

In addition to changing the management structure, the reformers sought to change hospital managers' incentives, with the objective of achieving better rationing and cost control (Crown Company Monitoring Advisory Unit 1996, 8). Arthur Anderson and Co (1987a) recommended to the Hospital and Related Services Taskforce that hospitals needed a "sharpened" management system. In particular, it advocated the use of "financial incentives to motivate" staff, which could involve "increasing salaries" for managers with the savings from improved management processes (Arthur Anderson and Co 1987a, I-2,40).

The focus on managers' incentives was apparent in CEO's employment contracts. The newly appointed CEOs had service and budget targets. One CEO performance target was better cost control, to eliminate the budget deficits seen under the AHBs. Performancebased bonuses could be a significant part of a CEO's remuneration package, up to $50 \%$ of their salary (Troughton 1993). Sharper incentives were expected to cascade through the hospital's management hierarchy to clinical staff, reinforcing the funder's objective of cost-efficiency (Vaithianathan 1999b). 
The newly appointed CEOs openly expressed their commitment to making services "more efficient", "reduc[ing] costs and break[ing] even" (Coast Health Care Limited 1994). Some CHEs cut costs dramatically. Capital Coast Health effected a "stunning financial turnaround" by cutting $\$ 33 \mathrm{M}$ in one financial year (Guyan 1998) $]^{3}$ Although most CHEs found deficits difficult to eliminate, many CEOs persisted. For example, Southern Health's CEO Anthea Green saw eliminating the deficit as "imperative" and CEO Paul Wylie stated "HealthLink South remains committed to the task [of improving value for money] and will continue to aggressively seek further efficiency gains" (Healthlink South 1996).

The reformers expected the managers' sharp incentives to cascade down the hospital hierarchy. This is consistent with Aghion \& Tirole's (1997) model of an organisational hierarchy through which authority may be delegated from superordinates to subordinates. CEOs were given strong incentives for cost control and tried to pass these incentives on, expecting staff to respond to opportunities to improve efficiency. One CEO, Paul Wylie, "required [staff] to re-examine not only what they do but how they do things and be ever vigilant for the opportunities to save costs" (Healthlink South 1996).

Internal process changes varied by $\mathrm{CHE}$, and included business plans, quality accreditation, establishing clinical management committees, developing care pathways, patient management systems, and reconfiguring hospital facilities to match the integration of primary and secondary services (Crown Company Monitoring Advisory Unit 1997, 11-33). But many initial efforts concentrated on cutting or contracting out support services rather than managing core clinical services (Ashton 1999, 142). CCMAU "observ[ed] substantial efficiency capture in internal processes" while "reducing direct personnel" (Crown Company Monitoring Advisory Unit 1997, 5).

Despite CCMAU's observations, it is questionable to what degree managers successfully shaped clinicians' incentives. "Senior management processes were weakened by the entry of new managers who were unused to operating in an environment where information

\footnotetext{
${ }^{3}$ Based on Crown Company Monitoring Advisory Unit (1997) figures, this saving was equivalent to just over one sixth of the entire sector deficit forecast in 1987/88.
} 
about costs and outputs was limited" (Ashton 1999, 142). As the general finance manager of Waikato Hospital commented in 1993, "The clinicians are responsible for prescribing treatments. It means that they drive the organisation in terms of costs. Last year one clinician spent $\$ 500,000$ on eight patients. Under the present system it is not possible to control and question their operations in an objective to see the components of such costs" (Alam \& Lawrence 1994, Stewart Lawrence \& Lowe 1994, 49).

CEOs were given sharp incentives for cost control and formal authority over allocation decisions. However, in practice it could be difficult for managers to secure sufficient information to make good decisions and to pass their incentives successfully to clinicians. Vaithianathan $(1999 b, 146)$ noted that "hospitals are unable to turn away patients who need immediate and urgent care. Therefore, managing volumes to contracted levels is almost impossible". That is, it was impossible for managers to refuse acute care in spite of budget pressures or performance incentives. This suggests that in some instances, CHE CEOs may have lacked sufficient real authority to control costs effectively.

The reforms did succeed in increasing the tension between some managers and clinical staff. The "professional and commercial cultures have come face to face in a way that has not happened before... At present there are serious tensions...". (Easton 1997). One source of tension was a conflict between managerial motives and the professional motives of medical specialists, where "as a rule doctors are professionally and legally inclined to provide patients with maximum quality care" (Evans (1984)). In some cases, however, "some Clinical Directors accepted the challenge of managing within limited budgets and co-operated with managers...[and] as a result, other senior medical staff stopped trusting these Clinical Directors [perceiving Clinical Directors as] having been captured by management" Health and Disability Commissioner (1998, 93).

In the case of patient deaths at Christchurch Hospital, the Health and Disability Commissioner concluded that "insufficient and inappropriate communication between management and staff resulted in failure to implement solutions identified by health professional staff" (Health and Disability Commissioner 1998, 86). The lack of communication and 
trust meant "patients did not receive appropriate quality care, particularly in the 1996 winter months".

Contrary to expectations, CHEs failed to secure sustained efficiency gains (Crown Company Monitoring Advisory Unit 1996, 7). In many cases, cost improvements were less than expected and in some instances hospital debt increased (Deloitte, Touche \& Tohmatsu 1996, Controller and Auditor General 1998). A report to the Ministry of Health concluded, "CHEs have been less successful at controlling costs in the short term than boards were in the period leading up to CHE establishment" (Deloitte et al. 1996). The report attributed this failing in part to "a weakening of processes after CHE establishment".

There is some evidence that cost increases were driven by patients being treated more intensively (Crown Company Monitoring Advisory Unit 1997) and due to falling clinical admission thresholds (Ministry of Health 1995, Vaithianathan 1999b). A key performance measure for CHEs monitored by CCMAU was patient length of stay. In 1997, CCMAU noted "reduced length of stay has been achieved through more expensive treatments and increased use of drugs (in part offering an explanation as to the failure of the sector to translate the process efficiencies achieved into financial gains)" (Crown Company Monitoring Advisory Unit 1997, 6).

The failure to achieve the reformers' expectations also appears to be due to the reforms disengaging clinicians from hospital management functions (Health and Disability Commissioner 1998). This may be because the desired incentives did not cascade down the hierarchy as expected, or because managers tried, and failed, to incentivise clinicians by exercising greater authority over them. In a survey on barriers to CHE CEOs' performance, a clinician/senior manager said "there are cultural barriers, the model requires new management tools... doctors are not usually by their nature team players. Therein lies an inherent conflict which poses a barrier to a CEO" (George, Inglis \& Rippin 1997). Managers frequently had difficulty controlling doctors, "Service Managers had little or no control over the clinical staff and in some cases Clinical Directors refused to report or cooperate with the Service Managers" (Health and Disability Commissioner 1998). 


\subsection{Literature}

This chapter shows how stronger, or 'sharper', incentives for a contractible task can worsen the outcomes of that task. Undesirable effects of sharp incentives have been posited in previous work, which is discussed below. That work does not adequately explain, in formal terms, the example of poor performance seen in the CHE reforms. That is, where rewarding a specific, measurable task appeared to worsen that task's outcome despite involving costless communication between interdependent agents.

\subsubsection{Undesirable performance effects of sharp incentives}

One strand of literature focuses on the nature of an activity and examines tasks that have multiple dimensions and jobs that involve multiple tasks. As noted in Chapter 1 , Holmström \& Milgrom (1991) argue that rewarding an agent for tasks with observable outcomes can encourage the agent to substitute effort inefficiently from tasks with unobservable, and therefore unrewarded, outcomes. This result may explain, for example, 'quality-quantity' tradeoffs where incentivising (observable) quantity targets may result in lower (unobservable) quality. This approach does not suggest how sharper incentives for an observable task could cause poorer performance for that same task.

Lazear (1989) shows how sharp incentives may reduce performance for a rewarded task and how soft incentives may improve collective performance $4^{4}$ In Lazear's (1989) model, relative performance pay creates an incentive for a worker to sabotage a peer's efforts. His model concentrates on horizontal coordination between peers, and how pay differentials may create competition between peers.

The model in this chapter differs from Lazear's (1989) model in a number of respects. First, we assume that the agents are part of an interdependent vertical hierarchy, rather

\footnotetext{
${ }^{4}$ Lazear (1989) refers to "pay compression" which is consistent with this chapter's use of soft incentives, but in a different environment. Pay compression describes the reduction of wage inequality in an interdependent group of workers who compete for high relative wages. Effort is costly, and workers allocate their effort between two tasks: producing output or sabotaging their peer's efforts. Pay compression reduces the payoff to sabotage. Lazear (1989) concludes that soft incentives, via wage equality, are efficient where sabotage is more ruinous to individual productive effort than are soft incentives.
} 
than co-workers that compete for relative wages. That is, it examines vertical coordination rather than horizontal coordination as in Lazear (1989). Second, we use a cheap talk model rather than assuming an agent takes a costly action that can result in a poor outcome. This avoids the interpretation of Lazear's (1989) model that sharp incentives may motivate sabotage and affect the availability of information. Rather, here we explicitly model the coordination process and how sharp incentives may erode credibility and impair the effectiveness of costless communication, which may result in poorer outcomes.

Behavioural economics provides another major strand of literature on the negative performance effects of sharp incentives. A standard result in the behavioural literature on sharp incentives is that a tradeoff may exist between intrinsic and extrinsic motives

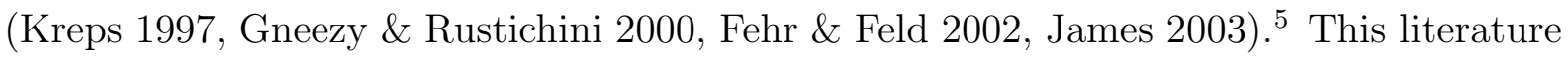
suggests that the net effect of stronger extrinsic motives on performance depends on the extent to which they crowd out intrinsic incentives. Sharpening existing extrinsic incentives, rather than newly introducing them, may reduce performance. This is possible if the extrinsic incentive is relatively small and its effect is outweighed by the corresponding effect of reduced intrinsic motivation.

Fehr \& Feld (2002) and Gneezy \& Rustichini (2000) argue that the interaction between extrinsic and intrinsic motives, and the net effect on performance, depends on whether extrinsic incentives are introduced or whether existing ones are strengthened. Gneezy \& Rustichini's (2000) experiments, for example, showed that large monetary incentives raised performance, but that people offered money did not always perform better than those paid nothing. In particular, the introduction of an extrinsic, monetary incentive could change "the perception of an incomplete contract" towards a focus on the monetary reward as a "reference point to decide the intensity of an activity" (Gneezy \& Rustichini $2000,804)$. They argue that the introduction of a monetary reward could replace intrinsic motivation with extrinsic motivation, and the net effect of a small monetary reward could

\footnotetext{
${ }^{5}$ Intrinsic incentives refer to an actor's internal motivation, or preferences, while extrinsic incentives refer to incentives created by others to compensate an actor for doing something that they would not otherwise choose to do.
} 
be weaker incentives and worse performance.

Kreps (1997) presents two informal arguments that stronger extrinsic incentives may lower performance, which reflect two definitions of intrinsic incentives. The first argument is based on "fuzzy extrinsic incentives". Additional effort may be a best response for risk-averse people to uncertainty about actual extrinsic incentives, for example where evaluation criteria are poorly defined. If incentives were clearer it would reduce a worker's uncertainty, so the agent "can put in just enough effort to stay safely employed... leading to a lower (average) effort level" (Kreps 1997, 362). In the second argument intrinsic incentives reflect a concern for others, but only for some types of relationship. A person may care about those close to the person, such as family, but not those people with whom the person has an arms-length relationships. Kreps (1997) argues that sharpening explicit incentives may make the person alter the person's assessment of the relationship from close to arms-length. If the resulting diffidence outweighs the positive effect of the extrinsic incentives, the extrinsic incentive could reduce net effort and performance.

James (2003) models the interaction of performance pay and consideration for others on effort. James (2003) includes consideration, or "intrinsic" motivation, in the agent's utility function. Performance pay may lower effort where an agent is intrinsically concerned with the principal's interests. It lowers effort if the direct benefit is exceeded by the indirect cost from lowering the principal's payoff.

The behavioural models above may explain poorer performance where extrinsic incentives are introduced, where the change in incentives is relatively minor, or the fundamental relationship is altered from close to arms-length which changes the relative importance of consideration for others. These scenarios, however, do not appear to correspond to the CHE reforms.

First, most CEOs were newly appointed and so had no prior attachment with the health sector. Second, where CEOs had private sector experience it is likely these CEOs were used to extrinsic performance incentives. Third, performance bonuses of up to $50 \%$ of a CEO's remuneration are unlikely to be considered as relatively small. In such a case, 
behavioural economics is likely to predict that the substantial extrinsic incentives would outweigh the crowding out of intrinsic motivation, thus increasing performance. Third, interactions between agents in a hierarchy is an important aspect of the performance of the organisation that is not captured in this behavioural literature: whether the incentives given to managers at the top of the hierarchy successfully passed down to, and motivated, medical specialists. This aspect is discussed further below, in the context of New Zealand's CHE reforms and with reference to a literature on authority in hierarchical organisations.

\subsubsection{New Zealand's CHE reforms}

In addition to these two strands of literature, and the broader literature reviewed in section 1.2, Vaithianathan analyses a range of economic incentives facing clinical decision makers and applies these insights to aspects of New Zealand's health sector reforms. Vaithianathan (1999b) and Vaithianathan (1999a) comment on the performance incentives created by the CHE reforms. She argues "that increasing hospital costs were due to the manager's inability to influence clinical decisions regarding the intensity of treatment". The role of information in conflicts between economic agents is central to one of Vaithianathan's (1999a) arguments. Vaithianathan (1999a) uses Aghion \& Tirole's (1997) framework of interdependence in hierarchies, discussed in Chapter 1, to examine the interaction of CHE managers and doctors.

Although our approach draws on the concept of an organisational hierarchy, our model in this chapter differs from Aghion \& Tirole's (1997) model. Aghion \& Tirole (1997) focuses on the conditions for optimal delegation, and formally models communication with "hard" or verifiable information. We focus on how the incentives created by a superordinate affects a subordinate's ability to acquire credible, but non-verifiable, information. That is, we model how the incentives created within an existing delegated structure affect the subordinate's ability, rather than their incentives, to acquire information.

Vaithianathan (1999a) uses Aghion \& Tirole's (1997) framework to discuss the effects of the CHE reforms on the allocation of formal and real authority within hospitals. Vaithi- 
anathan concludes that poorly informed CEOs lacked the real authority necessary to improve hospital efficiency, which affected their incentives to pursue this goal. Rather, CEOs were forced to "negotiate with doctors for any changes they require, and this exposes the CEO to potential 'holdup'" (Vaithianathan 1999a, 173).

This chapter focuses on a different, but complementary, question: how incongruous objectives between sub- and super-ordinates affect the communication, and credibility, of non-verifiable information in a multi-layered hierarchy. ${ }^{6}$ This chapter considers a situation with given allocations of formal authority and information, concentrating on communication and credibility, rather than on the generation of information. This chapter extends Vaithianathan's (1999a) analysis to model formally the interaction between a manager and a specialist, and the possible impacts of the CHE reforms on this interaction.

\subsubsection{Modelling concepts used in this chapter}

The model and solution approach presented in section 4.4 draw on cheap talk theory, which developed from the area of signalling theory. Signalling is often used to describe a form of costly communication where an informed party sends a message to a less informed party. If a message is verifiable, the sender can send a message that corresponds directly to the information. If the message is unverifiable, signals may still be useful if, due to the cost, only certain types of sender would want to send particular signals (Spence 1973, Kreps \& Sobel 1994). That is, a signal may gain credibility from the cost that different types of sender incur to send the signal rather than the content of the message per se. Crawford \& Sobel (1982) is a forerunner to the concept of cheap talk. It examines communication where signals are costless to the sender, and shows "how informative an equilibrium language can be, given the degree of conflict and of common interest" (Farrell 1993, 515).

\footnotetext{
${ }^{6}$ Dur \& Swank (2005) is an independent, but concurrent piece of work with this thesis, that considers the negative performance effects of divergent preferences. Dur \& Swank (2005) analyses delegation in cheap talk situations where the author assumes agents have some degree of bias against the principal's interests. Our model differs substantially from Dur \& Swank (2005). We endogenise the degree of bias between a funder and his immediate subordinate, an administrator. Further, we concentrate on how sharpening incentives for a given task may result in a poorer outcome of that same task.
} 
A cheap talk message is costless, non-verifiable and does not involve commitments. As such, it differs from a signal or a mechanism (Farrell \& Gibbons 1989). A signal may gain credibility from the cost of sending the message, as the willingness of different types of sender to incur such a cost may allow the receiver to discriminate between different types. A mechanism involves a commitment where "a mediator controls the communication and is committed to enforcing a given outcome as a function of the messages" (Farrell \& Gibbons 1989, 223). A cheap talk message has an impact to the extent that it affects the receiver's beliefs. Although the receiver may understand a message, he may not believe it: it is assumed that "messages are comprehensible and credibility is the only barrier to communication, out of equilibrium as well as in" (Farrell 1993, 520).

We use the equilibrium refinement of neologism-proofness to restrict our attention to plausible Bayesian Nash equilibria (BNE) of our cheap talk game.7 The neologism-proofness refinement discards a BNE if mutually beneficial communication could occur, but such communication (via a neologism) is not part of that BNE. Thus, a neologism-proof BNE (NPE) is robust to unexpected messages.

Farrell (1993) developed the concept of a neologism-proof equilibrium. Farrell $(1993,520)$ argues that the only barrier to communication in a cheap talk game is credibility. He requires that an equilibrium in such games be robust to mutually beneficial but unexpected messages, that is, credible neologisms. He motivates the use of neologisms by assuming that messages are communicated with a "rich language" and that this language could potentially be used to send an unexpected, but comprehensible, message (Matthews, Okuno-Fujiwara \& Postlewaite 1991, 248).8 A message sent using a rich language might have a literal, and therefore comprehensible, meaning.9 Farrell $(1993,520)$ also assumes

\footnotetext{
${ }^{7}$ For example, many games have implausible "babbling equilibria" (Myerson 1989, Farrell 1993). A babbling equilibrium is one where a receiver treats all messages as uninformative. Thus, the sender has a best response of randomising the messages sent, as the sender expects all messages to be ignored. Neologism-proofness can be used to rule out such implausible equilibria.

${ }^{8}$ The concepts of a rich language and messages with literal meanings, which are discussed below, are presented in an earlier piece of work by Myerson (1989). Myerson signals his awareness of Farrell's work, however, which was yet to be published.

${ }^{9}$ The use of a rich language differs from Crawford \& Sobel's (1982) landmark analysis of cheap talk games, where messages do not have a natural meaning but gain their meaning via their equilibrium use.
} 
that "for every non-empty subset [of types] $X \subseteq T$... there exists a message $n(X)$ that is unused in the equilibrium and whose literal meaning is that $t \in X$ ".

The credibility of an unexpected, non-verifiable message is crucial to meaningful communication. A neologism is credible if the sub-set of types who "prefer (strictly) this message to be believed over what they would get in the putative equilibrium" corresponds precisely to the senders' types (Matthews et al. 1991, 254). Such types would be strictly better off from using a neologism, rather than playing their putative equilibrium strategy, while types not in this sub-set would be weakly worse off from using this neologism. Farrell $(1993,581)$ refers to such a set of types as "self-signalling", and argues that the receiver should believe neologisms are used by such types. ${ }^{10}$ Self-signalling sets lend credibility to a non-verifiable message, and the existence of a credible neologism would disrupt a putative BNE. Thus, a BNE is neologism-proof if no credible neologisms are available.

One issue with using the BNE concept for analysing cheap talk games is that different messages may have the same meaning, so the specific words used in the messages are irrelevant to the players' payoffs. As one message may be interpreted as having the same meaning as an equivalent but different message, there may be numerous distinct equilibria. These equilibria differ only in the words in the message, rather than the information the message communicates. With an infinite message space, analysing message strategies complicates the analysis unnecessarily.

In response to the indeterminacy of equilibrium messages, Matthews et al. (1991) formalises Farrell's (1993) concepts of neologism-proofness and an equilibrium outcome. An outcome abstracts from specific messages and concentrates on payoff-relevant variables, such as types (Matthews et al. 1991). The equilibrium outcome approach simplifies the analysis of games where different messages can have the same meaning, by grouping together similar equilibria into an outcome. An equilibrium outcome is neologism-proof if

\footnotetext{
${ }^{10} \mathrm{~A}$ set of $\pi$ is self-signalling if the sub-set of types that would want to use a neologism equates precisely to that sub-set of types, given the sender prefers the payoff where the receiver believes the neologism and and accordingly plays his best response, rather than get their putative equilibrium payoff. That is, if the administrator adopts beliefs conditioned on the message, then the specialist would want to send such a message for $\pi$ in the self-signalling set, but would not want to send it for $\pi$ not in that set.
} 
the equilibria giving rise to it are neologism-proof (Matthews et al. 1991, 255). ${ }^{11}$

\subsection{Cheap talk: incentive congruence and credibility}

This section considers whether stronger incentives for cost control may worsen performance. It models an interaction between an administrator and a specialist as a cheap talk game. In such a model, sharp incentives could disrupt trust-based relationships within an organisation. In the context of the CHEs this could lead to an increase in costs. Section 4.4 .2 sets up the cheap talk game, and section 4.4.3 identifies potential BNE of this game. Section 4.4.4 uses the concept of neologism-proofness to refine the set of equilibrium candidates. Section 4.4.5 uses the results from these sections to consider explicitly the potential effects of sharpening an administrator's incentives on cost control and patient safety.

\subsubsection{A motivating example of harmful cost-cutting incentives}

We begin with a motivating example for the following theoretical model. The example shows that sharpening an administrator's incentives for cost control can cause higher costs.

Consider an administrator who rations costly treatment. Costlier treatments are safer, resulting in less harm to a patient if there is an adverse event. The administrator does not observe a person's risk of an adverse event. Suppose this risk can be high or low, and that the administrator knows that the two risk levels are equally likely.

The administrator works with a specialist who knows a patient's risk. The specialist can communicate this information to the administrator.

\footnotetext{
${ }^{11}$ The outcome approach is analogous to applying the revelation principle. The revelation principle "allows us, in any given economic situation, to make general statements about all possible communication mechanisms" (Myerson 2008, p2). The revelation principle states that, "any Bayesian Nash equilibrium of any Bayesian game can be represented by an incentive-compatible direct mechanism" (Gibbons 1992, p165). A direct mechanism is a type of "static Bayesian game in which each player's only action is to submit a claim about his or her type", and it is incentive-compatible where "truth telling is a Bayesian Nash equilibrium", that is, for each player's claim, $\mu_{i}$, about their type, $\pi_{i}$, is $\mu_{i}\left(\pi_{i}\right)=\pi_{i}$.
} 
In this illustration, the goals of the specialist and the administrator are incongruent. The administrator puts more emphasis on keeping costs down, while the specialist puts more emphasis on a patient's health 12

The following table presents the player's utilities for given outcomes. The numbers on the left denote the specialist's utility; higher numbers imply greater utility. ${ }^{13}$

Administrator's choice of treatment

\begin{tabular}{rr|c|c|c|}
\cline { 3 - 4 } & & low cost & moderate cost & high cost \\
\cline { 3 - 5 } Patient's risk & low risk & 4,5 & 8,3 & 6,2 \\
& high risk & 0,0 & 2,1 & 6,4 \\
\cline { 3 - 4 } & &
\end{tabular}

Table 4.1: Utilities for the specialist and administrator

When a patient's risk is low, the administrator prefers less intensive treatment than the specialist. When a patient's risk is high, both players prefer the high cost treatment.

If the specialist communicates the patient's risk honestly, the administrator would choose the low cost treatment when the risk is low. But if the administrator is uninformed about a particular patient, she would offer high cost treatment ${ }^{14}$ The specialist does better if the administrator is uninformed; and the administrator knows the specialist prefers her to remain uninformed.

In this illustration, the administrator's enthusiasm for cost-cutting is counter-productive. If the administrator cared less about cutting costs, it would align her incentives more closely to the specialist's preferences. Such an alignment lends credibility to the specialist's recommendations. With better information, the administrator can target treatment more appropriately, thereby lowering expected costs 15

\footnotetext{
${ }^{12}$ This assumption accords with the view that, "doctors generally have an innate tendency to overutilise health care resources on behalf of their patients" (Pauly 1980).

${ }^{13}$ This illustration is similar to example 4 in Farrell \& Rabin (1996).

${ }^{14}$ The administrator bases her decision on her expected utility. If the administrator is uninformed about a particular patient, we assume she believes low and high risk are equally likely, so her expected utility from low, moderate and high cost treatment in this example is $2.5,2$ and 3 , respectively.

${ }^{15}$ For example, if the administrator had the same preferences as the specialist, the administrator would
} 
In this illustration, if the administrator is uninformed, she errs on the side of caution and provides expensive treatment, that is sometimes excessive 16

\subsubsection{Play of the game}

Below we set out a cheap talk model to investigate how sharp incentives might result in poorer outcomes 17

There are two players in this model: an administrator, $A$, who uses a strategy denoted by $\tau$, and a specialist, $S$, who uses a strategy denoted by $\mu t^{18}$ At the beginning of the game, a player's expected payoff is as follows, where $i \in\{S, A\}$ :

$$
U_{i}(\mu, \tau)=E_{\pi}\left[u_{i}(\pi, \tau(\mu(\pi)))\right]
$$

Specialists may make a variety of recommendations in a hospital setting, such as about best practice guidelines, treatment protocols and advice on medical versus surgical intervention, given the private information they have about their patients. Administrators choose whether to accept or reject these recommendations. While this type of interaction may be quite general, we give it a specific medical interpretation in this chapter for concreteness.

prefer moderate treatment over low cost treatment when the risk was low. In this case, the specialist could honestly report the patient's condition and expect the administrator to believe it, as the administrator's (informed) decision would be consistent with the specialist's preferences. This would lower the total expected cost: when the patient's risk was low the administrator would approve the less costly moderate treatment rather than the high cost treatment; when the patient's risk was high costs would be unchanged.

${ }^{16}$ Chapter 3 showed that it may be optimal for a funder to increase the budget when the organisation's actual costs exceed its planned budget. This result suggests why a specialist might expect an administrator to be willing to provide the high cost treatment when the administrator is poorly informed. Although generous treatment might mean violating an initial budget constraint, the administrator may secure a budget increase from the funder when costs are higher than initially budgeted.

${ }^{17}$ As in Chapter 3 , for ease of reading, we assign genders to the two actors introduced in this chapter: a specialist (male) and a consumer (female).

${ }^{18}$ These strategies are defined on page 88 . 
The steps of the game are as follows:

1. Nature, $N$, chooses the specialist's type (i.e. the patient's condition), $\pi \sim H$.

2. A specialist, $S$, observes $\pi$. He chooses a message, $m \in M$.

3. An administrator, $A$, observes $m$ and chooses a treatment, $t \in T=\left\{t_{1}, t_{2}, t_{3}\right\}$.

4. Nature causes an adverse event with probability $\pi$ that results in harm of $\delta(t)$.

Figure 4.1 illustrates the steps of the game. The indicator function, $I$, denotes Nature's choice in the final step; $I$ equals one if an adverse event occurs and zero otherwise.

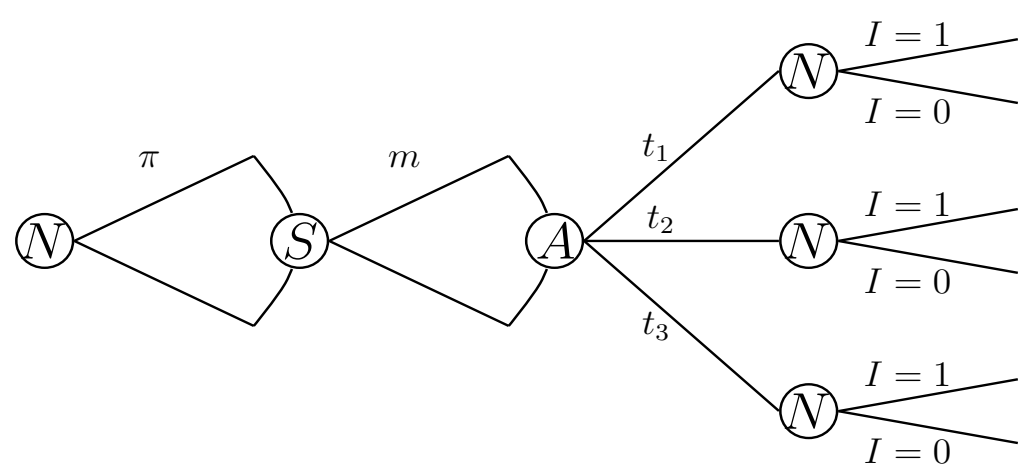

Figure 4.1: Game tree for cheap talk model

In the first step nature, $N$, selects the probability $\pi$ that a patient, $C$, would suffer an adverse event.19 In modelling terms, the patient's medical condition, $\pi$, may be thought of as a specialist's type 20 A patient's condition has a cumulative distribution function over $\pi \sim H$, where $\pi \in \Pi=[0,1]$. If an adverse event occurs, a patient suffers some

\footnotetext{
${ }^{19}$ For concreteness, the analysis refers to a specific patient, where $\pi$ reflects her underlying state of health or some risk factor that may affect how she responds to health care. The distribution of $\pi$ can be interpreted as how these risk factors are spread across the population.

${ }^{20}$ The patient is not a strategic actor in this model. While $\pi$ refers to the patient's characteristics, in game theoretic terms, $\pi$ may be interpreted as the specialist's type. $\pi$ is privately observed by the specialist, and it determines the specialist's information partition and payoffs. The administrator is uncertain about the specialist's type, but holds some beliefs about what the specialist knows, that is, about the specialist's type.
} 
harm. The severity of the harm depends on the treatment, $t$, she has received. Let $\delta(t)$ denote the players' evaluation of the harm a patient suffers from an adverse event.

The specialist, $S$, costlessly learns the patient's condition, $\pi$. The specialist's assessment of the patient's condition is private, non-contractible information; that is, $\pi$ is not verifiable. This information can be communicated to the administrator via a costless message, $m \in$ $M$. The specialist's messages are universally comprehensible but not necessarily credible. The specialist's strategy is a function $\mu: \Pi \rightarrow M$.

An administrator, $A$, receives the specialist's message, but does not observe $\pi$. She then authorises a particular treatment, $t$, according to her strategy, $\tau: M \rightarrow T$.

At the final stage of the game $\pi$ and $t$ are given, but Nature has not yet determined whether an adverse event occurs. The players' expected utilities at this point are:

$$
\begin{aligned}
& u_{S}=-[C(t)+\pi \delta(t)] \\
& u_{A}=-[(1+\theta) C(t)+\pi \delta(t)] .
\end{aligned}
$$

The disutility of treatment costs differ between the players because of the parameter $\theta$. This parameter reflects the greater relative weight that the administrator puts on treatment costs compared to the harm of an adverse event. The parameter $\theta$ drives a wedge between the specialist and administrator: as $\theta$ increases the administrator is more concerned with keeping treatment costs down than with patient safety.

We assume that more intensive treatment is more costly but reduces the harm if there is an adverse event. We consider three levels of treatment: $t_{1}, t_{2}$ and $t_{3}$. The treatments are ranked in order of increasing cost and safety. That is, a higher level of treatment implies more cost, $C\left(t_{i}\right)$, and less harm if there is an adverse event, $\delta\left(t_{i}\right)$, so $C\left(t_{1}\right)<C\left(t_{2}\right)<C\left(t_{3}\right)$ and $\delta\left(t_{3}\right)<\delta\left(t_{2}\right)<\delta\left(t_{1}\right)$. This relationship implies a trade-off between treatment cost and patient safety.

The following expressions are cost-benefit thresholds. They are used below to characterise the specialist's and administrator's preferred treatment for a given medical condition, $\pi .{ }^{21}$

\footnotetext{
${ }^{21}$ For example, $S$ prefers $t_{1}$ to $t_{2}$ if $C\left(t_{1}\right)+\pi \delta\left(t_{1}\right)<C\left(t_{2}\right)+\pi \delta\left(t_{2}\right) \Rightarrow \pi<\frac{C\left(t_{2}\right)-C\left(t_{1}\right)}{\delta\left(t_{1}\right)-\delta\left(t_{2}\right)}=\gamma_{12}$.
} 
Let $\gamma_{12}, \gamma_{13}$ and $\gamma_{23}$ be defined as follows:

$$
\gamma_{12}=\frac{C\left(t_{2}\right)-C\left(t_{1}\right)}{\delta\left(t_{1}\right)-\delta\left(t_{2}\right)}, \quad \gamma_{13}=\frac{C\left(t_{3}\right)-C\left(t_{1}\right)}{\delta\left(t_{1}\right)-\delta\left(t_{3}\right)}, \quad \gamma_{23}=\frac{C\left(t_{3}\right)-C\left(t_{2}\right)}{\delta\left(t_{2}\right)-\delta\left(t_{3}\right)} .
$$

The specialist's preferred treatment is as given by the following array ${ }^{22}$

$$
t_{S}^{\star}=\left\{\begin{array}{lll}
t_{1} & \text { if } & \pi \leq \gamma_{12} \\
t_{2} & \text { if } & \gamma_{12} \leq \pi \leq \gamma_{23} \\
t_{3} & \text { if } & \gamma_{23} \leq \pi
\end{array}\right.
$$

The specialist might want to use any of the three treatments, depending on $\pi{ }^{23}$ However, it may not be possible for the specialist to persuade the administrator to authorise his preferred treatment. For example, in a putative equilibrium where the administrator is only willing to authorise $t_{1}$ or $t_{3}$, then the specialist's threshold for choosing between these options is $\gamma_{13}$, as the specialist is unable to persuade the administrator to authorise $t_{2}$.

The administrator is more budget-conscious than the specialist, that is $0 \leq \theta$. If the administrator knew $\pi$, her preferred treatment would be as follows:

$$
t_{A}^{\star}=\left\{\begin{array}{lll}
t_{1} & \text { if } \quad \pi \leq(1+\theta) \gamma_{12} \\
t_{2} & \text { if } \quad(1+\theta) \gamma_{12} \leq \pi \leq(1+\theta) \gamma_{23} \\
t_{3} & \text { if } \quad(1+\theta) \gamma_{23} \leq \pi
\end{array}\right.
$$

We make the following two assumptions:

$$
\begin{aligned}
& 0<\gamma_{12}<\gamma_{23}<1 \\
& 0<P\left(\pi<\gamma_{12}\right)<P\left(\pi<\gamma_{13}\right)<P\left(\pi<\gamma_{23}\right)<1
\end{aligned}
$$

\footnotetext{
${ }^{22}$ When $\pi$ is equal to a threshold, the specialist would be indifferent between two treatments. As the distribution of $\pi$ is continuous, however, the probability that $\pi$ is exactly equal to a threshold is zero, $P\left(\pi=\gamma_{i j}\right)=0$ where $i, j \in\{1,2,3\}, i<j$.

${ }^{23}$ We make particular assumptions about the ordering of the thresholds and the cumulative probability distribution of $\pi$ below.
} 
The first assumption indicates that the cost-benefit threshold for a lower cost treatment is below that for a higher cost treatment. It also follows that $\gamma_{12}<\gamma_{13}<\gamma_{23}$. The second assumption indicates that it is possible that there are values of $\pi$ that lie in any of the intervals defined by the three thresholds. These two assumptions imply that the specialist may prefer any of the three levels of treatment, depending on the patient's medical condition, $\pi$.

\subsubsection{Bayesian Nash Equilibria}

Our first step is to consider the BNE of this game.

A BNE is a profile of strategies, where each player's strategy maximises their expected payoff given the other player's strategy. Let $\zeta_{S}$ and $\zeta_{A}$ denote the specialist's and administrator's strategy sets. The conditions for a strategy pair $(\mu, \tau)$ to be part of a BNE are as follows:24

$$
\begin{gathered}
U_{S}(\tilde{\mu}, \tau) \leq U_{S}(\mu, \tau), \quad \forall \tilde{\mu} \in \zeta_{S} \\
U_{A}(\mu, \tilde{\tau}) \leq U_{A}(\mu, \tau), \quad \forall \tilde{\tau} \in \zeta_{A}
\end{gathered}
$$

Conditions 4.7a and 4.7b require the equilibrium strategies to be best responses to each other:

$$
\begin{gathered}
\mu^{*} \in \arg \max _{\mu \in \zeta_{S}} U_{S}\left(\mu, \tau^{*}\right) \\
\tau^{*} \in \arg \max _{\tau \in \zeta_{A}} U_{A}\left(\mu^{*}, \tau\right) .
\end{gathered}
$$

Recall that the concept of an outcome allows us to abstract from differences between words in messages that convey the same meaning, and the corresponding strategies. An outcome is a mapping from the patient's medical conditions to treatments, $o: \Pi \rightarrow T$. It groups together different message and treatment strategies where they result in the same mapping from types to treatments. A BNE with a strategy profile $\left(\mu^{*}, \tau^{*}\right)$ generates an equilibrium outcome, $o^{*}(\cdot)$ :

$$
o^{*}(\cdot)=\tau^{*}\left(\mu^{*}(\cdot)\right)
$$

\footnotetext{
${ }^{24}$ These conditions follow a similar presentation to that given in Matthews et al. (1991).
} 


\section{BNE outcomes}

This section characterises the set of potential BNE outcomes in this model. We begin by outlining which outcomes are consistent with the specialist playing a best response. We then consider when an outcome is consistent with the administrator playing a best response.

For a given equilibrium, let $T_{0} \subseteq T$ describe a menu of treatments that the administrator is willing to fund. That is, $T_{0}$ is a set of treatments that the specialist could persuade the administrator to fund for a given strategy of the administrator, $\tau$ :

$$
T_{0}=\{t \in T \mid \exists m \in M, \tau(m)=t\} .
$$

If the administrator could be persuaded to fund any treatment, i.e. $T_{0}=T$, the specialist's utility maximising choice corresponds to the array in equation 4.4 on page 89.

The specialist may not always be able to convince the administrator to follow his ideal profile of treatments. In general terms, let $Z^{S}\left(t^{\prime}, T^{\prime}\right)$ represent the set of $\pi$ for which the specialist prefers $t^{\prime}$ to anything else in some set of treatments, $T^{\prime}$ :

$$
Z^{S}\left(t^{\prime}, T^{\prime}\right)=\left\{\pi \mid u_{S}(t, \pi) \leq u_{S}\left(t^{\prime}, \pi\right), \forall t \in T^{\prime}\right\}
$$

It is convenient to define the following terminology. The following sets divide up the support of $H$ with reference to the specialist's preferences: 25

$$
\begin{aligned}
Z_{1} & =\left\{\pi \mid 0 \leq \pi \leq \gamma_{12}\right\} \\
Z_{12} & =\left\{\pi \mid \gamma_{12} \leq \pi \leq \gamma_{13}\right\} \\
Z_{23} & =\left\{\pi \mid \gamma_{13} \leq \pi \leq \gamma_{23}\right\} \\
Z_{2} & =Z_{12} \cup Z_{23} \\
Z_{3} & =\left\{\pi \mid \gamma_{23} \leq \pi \leq 1\right\} .
\end{aligned}
$$

\footnotetext{
${ }^{25}$ The $\gamma_{i}$ thresholds are defined in array 4.4 .3 on page 90
} 
Table 4.2 shows the specialist's preferred treatment for any menu of treatments for various values of $T_{0}$ and $\pi$. That is, it shows when the specialist is playing a best response.

\begin{tabular}{|c|c|c|c|}
\hline When $T_{0}$ is... & $\begin{array}{c}S \text { prefers. . } \\
t_{1} \text { when }\end{array}$ & $t_{2}$ when & $t_{3}$ when \\
\hline$\left\{t_{1}, t_{2}, t_{3}\right\}$ & $\pi \in Z_{1}$ & $\pi \in Z_{2}$ & $\pi \in Z_{3}$ \\
\hline$\left\{t_{1}, t_{3}\right\}$ & $\pi \in Z_{1} \cup Z_{12}$ & & $\pi \in Z_{23} \cup Z_{3}$ \\
\hline$\left\{t_{1}, t_{2}\right\}$ & $\pi \in Z_{1}$ & $\pi \in Z_{2} \cup Z_{3}$ & \\
\hline$\left\{t_{2}, t_{3}\right\}$ & & $\pi \in Z_{1} \cup Z_{2}$ & $\pi \in Z_{3}$ \\
\hline$\left\{t_{1}\right\}$ & always & & \\
\hline$\left\{t_{2}\right\}$ & & always & \\
\hline$\left\{t_{3}\right\}$ & & & always \\
\hline
\end{tabular}

Table 4.2: S's preferred choices

The administrator's choice of $T_{0}$ is implied by her strategy, and her preferred treatment is characterised by array 4.5. However, as the administrator does not directly observe the patient's medical condition, $\pi$, that array does not characterise her actual decision. Rather, her preferred choice depends on what she believes about $\pi$ given the specialist's message.

Let $Z^{A}\left(t^{\prime}, T\right)$ be the set of $\pi$ for which the administrator would prefer $t^{\prime}$ out of the set of possible treatments, $T$ :

$$
Z^{A}\left(t^{\prime}, T\right)=\left\{\pi \mid E\left[u_{A}(t, \pi)\right] \leq E\left[u_{A}\left(t^{\prime}, \pi\right)\right], \forall t \in T\right\}
$$

In equilibrium, the administrator bases her decision on what she can infer from the specialist's message, given the menu of treatments the specialist can make recommendations from, $T_{0}$. The administrator's choice of $t^{\prime}$ is consistent with her also playing a best response under the following condition:

$$
t^{\prime} \in \arg \max _{t \in T} E\left[u_{A}(t, \pi) \mid \pi \in Z^{S}\left(t^{\prime}, T_{0}\right)\right]
$$


Under this condition, if the administrator believes that $\pi \in Z^{S}\left(t_{i}, T_{0}\right)$, then she will authorise $t_{i}$. Hence, the choice of $t^{\prime}$, for a given $\pi$ and $T_{0}$, is consistent with both players using a best response if $E\left[\pi \mid \pi \in Z^{S}\left(t^{\prime}, T_{0}\right)\right] \in Z^{A}\left(t^{\prime}, T\right)$.

Table 4.3 on suggests BNE for various treatment menus, and shows the conditions that must be satisfied for a given $T_{0}$ to be consistent with a BNE. When these conditions are satisfied, the menu $T_{0}$ characterises the equilibrium outcome.

\begin{tabular}{|c|c|c|c|}
\hline $\begin{array}{l}\text { When } T_{0} \\
\text { is. . }\end{array}$ & \multicolumn{2}{|c|}{$\begin{array}{c}\text { if } S \text { plays a best response } \\
\text { the outcome is... }\end{array}$} & $\begin{array}{l}\text { which is consistent with } A \\
\text { also playing a best response if. . }\end{array}$ \\
\hline$\left\{t_{1}, t_{2}, t_{3}\right\}$ & $o^{*}(\pi)=\left\{\begin{array}{l}t_{1} \\
t_{2} \\
t_{3}\end{array}\right.$ & $\begin{array}{c}\pi \leq \gamma_{12} \\
\gamma_{12} \leq \pi \leq \gamma_{23} \\
\gamma_{23} \leq \pi\end{array}$ & $\begin{array}{c}E\left[\pi \mid \pi \in Z_{1}\right] \leq(1+\theta) \gamma_{12} \\
(1+\theta) \gamma_{12} \leq E\left[\pi \mid \pi \in Z_{2}\right] \leq(1+\theta) \gamma_{23} \\
(1+\theta) \gamma_{23} \leq E\left[\pi \mid \pi \in Z_{3}\right]\end{array}$ \\
\hline$\left\{t_{1}, t_{3}\right\}$ & $o^{*}(\pi)=\left\{\begin{array}{l}t_{1} \\
t_{3}\end{array}\right.$ & $\begin{array}{l}\pi \leq \gamma_{13} \\
\gamma_{13} \leq \pi\end{array}$ & $\begin{array}{l}E\left[\pi \mid \pi \in Z_{1} \cup Z_{12}\right] \leq(1+\theta) \gamma_{12} \\
(1+\theta) \gamma_{23} \leq E\left[\pi \mid \pi \in Z_{23} \cup Z_{3}\right]\end{array}$ \\
\hline$\left\{t_{1}, t_{2}\right\}$ & $o^{*}(\pi)=\left\{\begin{array}{l}t_{1} \\
t_{2}\end{array}\right.$ & $\begin{array}{l}\pi \leq \gamma_{12} \\
\gamma_{12} \leq \pi\end{array}$ & $\begin{array}{c}E\left[\pi \mid \pi \in Z_{1}\right] \leq(1+\theta) \gamma_{12} \\
(1+\theta) \gamma_{12} \leq E\left[\pi \mid \pi \in Z_{2} \cup Z_{3}\right] \leq(1+\theta) \gamma_{23}\end{array}$ \\
\hline$\left\{t_{2}, t_{3}\right\}$ & $o^{*}(\pi)=\left\{\begin{array}{l}t_{2} \\
t_{3}\end{array}\right.$ & $\begin{array}{l}\pi \leq \gamma_{23} \\
\gamma_{23} \leq \pi\end{array}$ & $\begin{array}{c}(1+\theta) \gamma_{12} \leq E\left[\pi \mid \pi \in Z_{1} \cup Z_{2}\right] \leq(1+\theta) \gamma_{23} \\
(1+\theta) \gamma_{23} \leq E\left[\pi \mid \pi \in Z_{3}\right]\end{array}$ \\
\hline$\left\{t_{1}\right\}$ & $o^{*}(\pi)=t_{1}$ & always & $E[\pi] \leq(1+\theta) \gamma_{12}$ \\
\hline$\left\{t_{2}\right\}$ & $o^{*}(\pi)=t_{2}$ & always & $(1+\theta) \gamma_{12} \leq E[\pi] \leq(1+\theta) \gamma_{23}$ \\
\hline$\left\{t_{3}\right\}$ & $o^{*}(\pi)=t_{3}$ & always & $(1+\theta) \gamma_{23} \leq E[\pi]$ \\
\hline
\end{tabular}

Table 4.3: Conditions for $T_{0}$ to be consistent with a BNE outcome

In some equilibrium outcomes, the administrator is unwilling to fund some treatments. For example, in an equilibrium outcome with $T_{0}=\left\{t_{1}, t_{2}\right\}$ the specialist must choose between $t_{1}$ or $t_{2}$ : he will recommend $t_{1}$ when $\pi \in Z_{1}$, and $t_{2}$ otherwise.

Given the menu of treatments $T_{0}=\left\{t_{1}, t_{3}\right\}$, in equilibrium, the specialist recommends $t_{1}$ 
when $\pi \in Z_{1} \cup Z_{12}$, and $t_{3}$ otherwise. If the specialist recommends $t_{3}$, for example, the administrator can infer that $\pi \in Z^{S}\left(t_{3}, T_{0}=\left\{t_{1}, t_{3}\right\}\right)=Z_{23} \cup Z_{3}=\left[\gamma_{13}, 1\right]$. Authorising $t_{3}$ over any other treatment in $T$ would be consistent with the administrator playing a best response if $(1+\theta) \gamma_{23} \leq E\left[\pi \mid \pi \in Z_{23} \cup Z_{3}\right]$. This requirement is captured by the condition in the fifth row of Table 4.3 .

Not all of the conditions in Table 4.3 are substantive restrictions. For example, $E[\pi \mid \pi \in$ $\left.Z_{1}\right] \leq(1+\theta) \gamma_{12}$ is always true: the specialist recommends $t_{1}$ in this outcome when $\pi \in Z_{1}$ and the administrator is always willing to follow this recommendation.

The conditions in Table 4.3 are not always mutually exclusive, so the model does not necessarily have a unique BNE outcome. For example, a BNE outcome with either $T_{0}=$ $\left\{t_{1}, t_{2}\right\}$ or $T_{0}=\left\{t_{1}, t_{3}\right\}$ is possible for a certain range of parameters.

The outcomes suggest that a range of treatment rationing could potentially occur, consistent with the expectations of the CHE reformers or the actual CHE performance. Some would be consistent with low costs, $T_{0}=\left\{t_{1}\right\}$, a responsive treatment regime, $T_{0}=\left\{t_{1}, t_{2}, t_{3}\right\}$ or high costs, $T_{0}=\left\{t_{3}\right\}$. Below we narrow down the set of equilibrium outcomes to those that are plausible, and investigate how sharp incentives might result in an outcome with high costs, as in the second criticism of the CHE reforms.

\subsubsection{Neologism-proof Bayesian Nash equilibrium outcomes}

Although the outcomes specified in Table 4.3 are consistent with a BNE, they are not all plausible. In some BNE outcomes, there would be circumstances where the specialist fails to recommend an alternative to the putative equilibrium treatment, even though that alternative would be better for both players.

This section refines the set of BNE outcomes identified in section 4.4 .3 by applying the equilibrium refinement of neologism-proofness. Neologism-proofness strengthens the requirements for a BNE outcome to be considered plausible. A BNE outcome is neologismproof when the specialist expects the administrator to believe all credible neologisms, but 
there is no set of $\pi$ for which the specialist may credibly send a neologism that would disrupt the equilibrium.

Below, we set out the conditions for the putative BNE outcomes identified above to be neologism-proof. Let $Z^{S}\left(\tilde{t}, T_{0}\right)$ represent the set of $\pi$ for which the specialist prefers an alternative treatment, $\tilde{t} \notin T_{0}$, to anything else in the menu of treatments, $T_{0}:{ }^{26}$

$$
Z^{S}\left(\tilde{t}, T_{0}\right)=\left\{\pi \mid u_{S}(t, \pi) \leq u_{S}(\tilde{t}, \pi)\right\}
$$

The specialist would only want to use a neologism, instead of his putative equilibrium message, if he expects the administrator to believe that neologism.

In the extreme case, when $\theta=0$ and $Z^{A}(\tilde{t}, T)=Z^{S}\left(\tilde{t}, T_{0}\right)$ in equilibrium, then any neologism recommending $\tilde{t}$ is credible. The specialist would want to use such a neologism when $\pi \in Z^{S}\left(\tilde{t}, T_{0}\right)$, and the administrator is willing to follow this recommendation as $E[\pi \mid m] \in Z^{A}$. The specialist would want to deviate from his putative equilibrium strategy when $\pi \in Z^{S}\left(\tilde{t}, T_{0}\right)$, as he expects the administrator would believe the neologism and act accordingly.

More generally, if $\theta>0$ then $Z^{A}(\tilde{t}, T) \neq Z^{S}\left(\tilde{t}, T_{0}\right)$. The administrator does not observe $\pi$ and learns about it indirectly, and possibly imprecisely, from the specialist's message. The administrator can only deduce that $\pi \in Z^{S}\left(\tilde{t}, T_{0}\right)$. Consequently, her interpretation of the message will be $E[\pi \mid m]=E\left[\pi \mid \pi \in Z^{S}\left(\tilde{t}, T_{0}\right)\right]$. If $E[\pi \mid m] \in Z^{A}(\tilde{t}, T)$, then the neologism is credible, and the administrator would authorise $\tilde{t}$. Thus, in the case where $\theta>0$, the specialist would prefer to deviate from the putative equilibrium message and use a neologism, upsetting the putative equilibrium.

The condition below summarises when a neologism can upset a proposed BNE with $T_{0}:{ }^{27}$

${ }^{26}$ In order to evaluate the BNE outcomes from section $4.4 .3, Z^{S}$ may also be expressed as follows:

$$
Z^{S}\left(\tilde{t}, T_{0}\right)=\left\{\pi \mid u_{S}(o(\pi), \pi) \leq u_{S}(\tilde{t}, \pi) \forall t \in T_{0}\right\}
$$

${ }^{27}$ This condition requires the administrator to authorise $\tilde{t}$ rather than the putative equilibrium choice, $o^{*}(\pi)$, for some given $T_{0}$ as it is utility maximising to do so:

$$
\tilde{t} \in \arg \max _{t \in T} E\left[u_{A}(t, \pi) \mid u_{S}(o(\pi), \pi) \leq u_{S}(\tilde{t}, \pi)\right] .
$$




$$
E\left[\pi \mid \pi \in Z^{S}\left(\tilde{t}, T_{0}\right)\right] \in Z^{A}(\tilde{t}, T), \exists \tilde{t} \notin T_{0} .
$$

Equation (4.16) indicates that authorising the alternative treatment recommended by a neologism is consistent with both players maximising their expected utility. If this condition is satisfied, the putative equilibrium outcome is not neologism-proof and is therefore judged implausible.

For example, in the potential BNE outcome with $T_{0}=\left\{t_{1}\right\}$, the only treatment ever provided is $t_{1}$. However, for $\pi \in Z_{2} \cup Z_{3}$, the specialist prefers $t_{2}$ to $t_{1}$. Although this alternative is not part of the putative treatment menu, he could use an unexpected message to recommend it. The administrator prefers $t_{2}$ for higher values of $\pi$. The neologism would be persuasive if $(1+\theta) \gamma_{12} \leq E\left[\pi \mid \pi \in Z^{S}\left(t_{2}, T_{0}\right)\right]{ }^{28}$

Below, equations (4.15) and (4.16) are used to characterise the conditions under which the potential BNE outcomes from Table 4.3 in section 4.4 .3 are neologism-proof.

Table 4.4 shows when the specialist may use a credible neologism to suggest the only unexpected treatment. The set of $\pi$ that satisfies the conditions in the table is a selfsignalling set of $\pi$ for a given $T_{0}$.

\begin{tabular}{|l|c|c|c|}
\hline When & \multicolumn{2}{|c|}{$S$ prefers to use a neologism } & and which is credible to $A$ and affects \\
$T_{0}$ is... & $\tilde{t} \notin T_{0}$ & when $\pi \in Z^{S}\left(\tilde{t}, T_{0}\right)$ & her choice if $E\left[\pi \mid \pi \in Z^{S}\left(\tilde{t}, T_{0}\right)\right] \in Z^{A}(\tilde{t}, T)$ \\
\hline$\left\{t_{1}, t_{3}\right\}$ & $t_{2}$ & $\gamma_{12} \leq \pi \leq \gamma_{23}$ & $(1+\theta) \gamma_{12} \leq E\left[\pi \mid \pi \in Z_{2}\right] \leq(1+\theta) \gamma_{23}$ \\
\hline$\left\{t_{1}, t_{2}\right\}$ & $t_{3}$ & $\gamma_{23} \leq \pi$ & $(1+\theta) \gamma_{23} \leq E\left[\pi \mid \pi \in Z_{3}\right]$ \\
\hline$\left\{t_{2}, t_{3}\right\}$ & $t_{1}$ & $\pi \leq \gamma_{12}$ & $E\left[\pi \mid \pi \in Z_{1}\right] \leq(1+\theta) \gamma_{12}{ }^{\dagger}$ \\
\hline
\end{tabular}

$\dagger$ Denotes the condition is always true.

Table 4.4: Conditions for deviating from a potential BNE outcome with $\left|T_{0}\right|=2$

There can never be an NPE with $T_{0}=\left\{t_{2}, t_{3}\right\}$, as the condition for a credible neologism is always satisfied. The specialist could use a neologism if $E\left[\pi \mid \pi \in Z_{1}\right] \leq(1+\theta) \gamma_{12}$ to

\footnotetext{
${ }^{28}$ However, the neologism would not be persuasive if $E\left[\pi \mid \pi \in Z^{S}\left(t_{2}, T_{0}\right)\right] \leq(1+\theta) \gamma_{12}$, that is, if $E\left[\pi \mid \pi \in Z^{S}\left(t_{2}, T_{0}\right)\right] \notin Z^{A}\left(t_{2}, T_{0}\right)$. In this circumstance, the administrator does not believe that the specialist would only want to use a neologism recommending $t_{2}$ when $\pi \in Z^{A}\left(t_{2}, T_{0}\right)$.
} 
recommend the unexpected treatment, $t_{1}$. As $0 \leq \theta$, this is always true. The intuition here is that the specialist can always convince the administrator to approve a lower cost treatment. Given the specialist's preference for more costly treatment, the administrator knows that the specialist would only recommend a lower cost treatment if it were also in the administrator's interests to authorise this treatment.

When a proposed BNE only has one level of treatment, then the specialist can express two kinds of neologism. Table 4.5 reports the conditions required for a credible neologism that would cause a deviation from a particular putative equilibrium outcome, with $\left|T_{0}\right|=1$.

\begin{tabular}{|c|c|c|c|c|}
\hline $\begin{array}{l}\text { When } \\
T_{0} \text { is. . }\end{array}$ & \multicolumn{2}{|c|}{$\begin{array}{l}S \text { prefers to use a neologism } \\
\tilde{t} \notin T_{0} \quad \text { when } \pi \in Z^{S}\left(\tilde{t}, T_{0}\right)\end{array}$} & \multicolumn{2}{|l|}{$\begin{array}{c}\text { and which is credible to } A \text { and affects } \\
\text { her choice if } E\left[\pi \mid \pi \in Z^{S}\left(\tilde{t}, T_{0}\right)\right] \in Z^{A}(\tilde{t}, T)\end{array}$} \\
\hline$\left\{t_{1}\right\}$ & $\begin{array}{l}t_{2} \\
t_{3}\end{array}$ & $\begin{array}{l}\gamma_{12} \leq \pi \\
\gamma_{13} \leq \pi\end{array}$ & $\begin{array}{c}(1+\theta) \gamma_{12} \leq E\left[\pi \mid \pi \in Z_{2} \cup Z_{3}\right] \leq(1+\theta) \gamma_{23} \\
(1+\theta) \gamma_{23} \leq E\left[\pi \mid \pi \in Z_{23} \cup Z_{3}\right]\end{array}$ & \\
\hline$\left\{t_{2}\right\}$ & $\begin{array}{l}t_{1} \\
t_{3}\end{array}$ & $\begin{array}{l}\pi \leq \gamma_{12} \\
\gamma_{23} \leq \pi\end{array}$ & $\begin{array}{l}E\left[\pi \mid \pi \in Z_{1}\right] \leq(1+\theta) \gamma_{12} \\
(1+\theta) \gamma_{23} \leq E\left[\pi \mid \pi \in Z_{3}\right]\end{array}$ & $\dagger$ \\
\hline$\left\{t_{3}\right\}$ & $\begin{array}{l}t_{2} \\
t_{1}\end{array}$ & $\begin{array}{l}\pi \leq \gamma_{23} \\
\pi \leq \gamma_{13}\end{array}$ & $\begin{array}{c}(1+\theta) \gamma_{12} \leq E\left[\pi \mid \pi \in Z_{1} \cup Z_{2}\right] \leq(1+\theta) \gamma_{23} \\
E\left[\pi \mid \pi \in Z_{1} \cup Z_{12}\right] \leq(1+\theta) \gamma_{12}\end{array}$ & \\
\hline
\end{tabular}

$\dagger$ Denotes the condition is always true.

$\dagger^{\dagger}$ Denotes either condition 1 is true, or if it is not, then condition 2 is true.

Table 4.5: Conditions for deviating from a potential BNE outcome with $\left|T_{0}\right|=1$

There can never be an NPE with $T_{0}=\left\{t_{2}\right\}$ or $T_{0}=\left\{t_{3}\right\}$. The specialist can always use a credible neologism to convince the administrator to switch to a lower cost treatment; namely, when $\pi \in Z_{1}$ for the putative outcome with $T_{0}=\left\{t_{2}\right\}$ and $\pi \in Z_{1} \cup Z_{2}$ for the outcome with $T_{0}=\left\{t_{3}\right\}$. For example, with $T_{0}=\left\{t_{3}\right\}$, the specialist can certainly devise a credible neologism when $\pi \in Z_{1} \cup Z_{2}$.

But which neologism disrupts a putative equilibrium depends on the distribution of $\pi$. If $(1+\theta) \gamma_{12} \leq E\left[\pi \mid \pi \in Z_{1} \cup Z_{2}\right]$, then the administrator will authorise $t_{2}$ when the specialist wants to recommend it. But this condition is not always true. If this condition is not true, 
so $E\left[\pi \mid \pi \in Z_{1} \cup Z_{2}\right] \leq(1+\theta) \gamma_{12}$, then a neologism recommending $t_{1}$ would be credible. That is, if $E\left[\pi \mid \pi \in Z_{1} \cup Z_{2}\right] \leq(1+\theta) \gamma_{12}$ then $E\left[\pi \mid \pi \in Z_{1} \cup Z_{12}\right] \leq(1+\theta) \gamma_{12}$. Hence, the specialist can always use some neologism to disrupt the putative outcome $T_{0}=\left\{t_{3}\right\}$ when $\pi \in Z_{1} \cup Z_{2}$.

If $T_{0}=\left\{t_{1}\right\}$ then the specialist can sometimes convince the administrator to deviate from the putative equilibrium. When $\pi \in Z_{2} \cup Z_{3}$, the specialist prefers $t_{2}$ over the putative equilibrium treatment of $t_{1}$. If $(1+\theta) \gamma_{12} \leq E\left[\pi \mid \pi \in Z_{2} \cup Z_{3}\right]$, then the administrator is willing to deviate from the putative equilibrium outcome when the specialist wants to use a neologism recommending $t_{2}$. However, there is not always a self-signalling set of $\pi$. That is, the specialist cannot always use a neologism that is credible to the administrator and which would disrupt the potential BNE outcome with $T_{0}=\left\{t_{1}\right\}$, as it is possible that $E\left[\pi \mid \pi \in Z_{2} \cup Z_{3}\right]<(1+\theta) \gamma_{12}$

Below, we discard the implausible BNE outcomes, that is, those that could be disrupted by credible deviations, as specified in Tables 4.4 and 4.5 . Table 4.6 on page 99 reports the set of conditions required for a neologism-proof BNE outcome for different values of $T_{0}$, that is, a NPE outcome ${ }^{29}$ For ease of reference, we label the four potential NPE outcomes as: $A=\left\{t_{1}, t_{2}, t_{3}\right\}, B=\left\{t_{1}, t_{3}\right\}, C=\left\{t_{1}, t_{2}\right\}$ and $D=\left\{t_{1}\right\}$; the substantive conditions for each outcome are labelled accordingly in the table below.

There can never be an NPE with $T_{0}=\left\{t_{2}, t_{3}\right\}, T_{0}=\left\{t_{2}\right\}$, or $T_{0}=\left\{t_{3}\right\}$ With these selections of $T_{0}$, the specialist can always devise a credible neologism that would cause the administrator to deviate from the putative equilibrium outcome.

\footnotetext{
${ }^{29}$ The additional conditions in this table over Table 4.3 reflect the stronger conditions for an NPE.

${ }^{30}$ Matthews et al. (1991) offers the alternative, but more complex, refinements of announcement proof (AP) and strong announcement proof (SAP) equilibrium. These concepts allow for more sophisticated messages. Where the sender only communicates a message about a single unexpected alternative, however, neologism-proofness and AP/SAP give equivalent results.

Where more sophisticated deviations are possible, the AP/SAP concepts can be used to provide greater refinement. For example, when $T_{0}=\left\{t_{3}\right\}$, a neologism indicates a preference for either $t_{1}$ or $t_{2}$, i.e. $\pi \in Z_{1} \cup Z_{2}$. With an announcement the specialist might indicate a preference for $t_{2}$ over $t_{1}$, i.e. when $\pi \in Z_{2}$. This could provide the administrator with greater information on which to condition her expectations. We would still rule out $T_{0}=\left\{t_{3}\right\}$ as a credible outcome under the AP/SAP criterion.

The NPE concept yields existence and permits our novel result, so we adopt the less restrictive approach.
} 


\begin{tabular}{|c|c|c|c|c|}
\hline $\begin{array}{l}\text { When } T_{0} \\
\text { is... }\end{array}$ & $o(\cdot)$ & & and is robust to neologisms if. & \\
\hline$\left\{t_{1}, t_{2}, t_{3}\right\}$ & $o^{*}(\pi)=\left\{\begin{array}{l}t_{1} \\
t_{2} \\
t_{3}\end{array}\right.$ & $\begin{array}{l}\pi \leq \gamma_{12} \\
2 \leq \pi \leq \gamma_{23} \\
\gamma_{23} \leq \pi\end{array}$ & $\begin{array}{c}E\left[\pi \mid \pi \in Z_{1}\right] \leq(1+\theta) \gamma_{12} \\
(1+\theta) \gamma_{12} \leq E\left[\pi \mid \pi \in Z_{2}\right] \leq(1+\theta) \gamma_{23} \\
(1+\theta) \gamma_{23} \leq E\left[\pi \mid \pi \in Z_{3}\right]\end{array}$ & $\begin{array}{l}\dagger \\
{ }^{A} 1 \\
{ }^{+}\end{array}$ \\
\hline$\left\{t_{1}, t_{3}\right\}$ & $o^{*}(\pi)=\left\{\begin{array}{l}t_{1} \\
\end{array}\right.$ & $\begin{array}{l}\pi \leq \gamma_{13} \\
\gamma_{13} \leq \pi\end{array}$ & $\begin{array}{c}E\left[\pi \mid \pi \in Z_{1} \cup Z_{12}\right] \leq(1+\theta) \gamma_{12} \\
E\left[\pi \mid \pi \in Z_{2}\right] \leq(1+\theta) \gamma_{12} \\
(1+\theta) \gamma_{23} \leq E\left[\pi \mid \pi \in Z_{23} \cup Z_{3}\right]\end{array}$ & $\begin{array}{l}\dagger B \\
B 1 \\
B 2\end{array}$ \\
\hline$\left\{t_{1}, t_{2}\right\}$ & $o^{*}(\pi)=\left\{\begin{array}{l}t_{1} \\
t_{2}\end{array}\right.$ & $\begin{array}{l}\pi \leq \gamma_{12} \\
\gamma_{12} \leq \pi\end{array}$ & $\begin{array}{c}E\left[\pi \mid \pi \in Z_{1}\right] \leq(1+\theta) \gamma_{12} \\
(1+\theta) \gamma_{12} \leq E\left[\pi \mid \pi \in Z_{2} \cup Z_{3}\right] \leq(1+\theta) \gamma_{23} \\
E\left[\pi \mid \pi \in Z_{3}\right] \leq(1+\theta) \gamma_{23}\end{array}$ & ${ }^{\dagger}$ \\
\hline$\left\{t_{2}, t_{3}\right\}$ & $o^{*}(\pi)=\left\{\begin{array}{l}t_{2} \\
t_{3}\end{array}\right.$ & $\begin{array}{l}\pi \leq \gamma_{23} \\
\gamma_{23} \leq \pi\end{array}$ & $\begin{array}{c}(1+\theta) \gamma_{12} \leq E\left[\pi \mid \pi \in Z_{1}\right] \\
(1+\theta) \gamma_{12} \leq E\left[\pi \mid \pi \in Z_{1} \cup Z_{2}\right] \leq(1+\theta) \gamma_{23} \\
(1+\theta) \gamma_{23} \leq E\left[\pi \mid \pi \in Z_{3}\right]\end{array}$ & $\dagger$ \\
\hline$\left\{t_{1}\right\}$ & $o^{*}(\pi)=t_{1}$ & always & $\begin{array}{c}E[\pi] \leq(1+\theta) \gamma_{12} \\
E\left[\pi \mid \pi \in Z_{2} \cup Z_{3}\right] \leq(1+\theta) \gamma_{12} \\
E\left[\pi \mid \pi \in Z_{23} \cup Z_{3}\right] \leq(1+\theta) \gamma_{23}\end{array}$ & $\begin{array}{l}\dagger D \\
D 1 \\
D 2\end{array}$ \\
\hline$\left\{t_{2}\right\}$ & $o^{*}(\pi)=t_{2}$ & always & $\begin{array}{c}(1+\theta) \gamma_{12} \leq E\left[\pi \mid \pi \in Z_{1}\right] \leq(1+\theta) \gamma_{23} \\
E\left[\pi \mid \pi \in Z_{3}\right] \leq(1+\theta) \gamma_{23}\end{array}$ & $\dagger$ \\
\hline$\left\{t_{3}\right\}$ & $o^{*}(\pi)=t_{3}$ & always & $(1+\theta) \gamma_{23} \leq E\left[\pi \mid \gamma_{23} \leq \pi\right]$ & $\dagger$ \\
\hline
\end{tabular}

$\dagger$ denotes the condition is always true. $\quad \dagger^{\dagger}$ denotes the condition is never true.

$\dagger B$ denotes the condition is true if $\mathrm{B} 1$ is true. ${ }^{\dagger D}$ denotes the condition is true if $\mathrm{D} 1$ is true.

Table 4.6: Conditions for $T_{0}$ to be consistent with a NPE outcome

When $\theta=0$, then the administrator and the specialist have the same preferences, and only $T_{0}=\left\{t_{1}, t_{2}, t_{3}\right\}$ is neologism-proof. In this outcome, there are no deviations from the putative equilibrium that the specialist would be tempted to make. Furthermore, when $\theta=0$ none of the potential outcomes with restricted treatment sets is neologism- 
proof. That is, when the two players' interests are perfectly aligned, it would be in the administrator's best interests to follow any recommendation the specialist makes.

The conditions in Table 4.6 are mutually exclusive, so the model has a unique NPE outcome if one exists (we demonstrate this below). This implies that we can identify a particular equilibrium outcome, for given parameter values. In section 4.4.5, we examine how changes in these parameter values, such as sharpening the administrator's incentives, could cause a shift from one outcome to another, resulting in higher treatment costs.

\section{Uniqueness and existence of an NPE outcome}

Before proceeding, we show that a NPE outcome is unique over a generic range of parameter values and the circumstances under which existence can be guaranteed.

For an equilibrium outcome with a given menu $T_{0}$, we require that there be no credible neologism that will cause the administrator to choose to authorise $\tilde{t} \notin T_{0}$, while a message recommending $t_{i} \in T_{0}$ elicits the desired response. The proof for each proposition mirrors the substantive necessary conditions for each type of NPE outcome indicated in Table 4.6. The propositions below express these conditions in terms of the range of $\theta$ over which the conditions would be satisfied.

The variables below represent thresholds at which the administrator is indifferent about funding a given treatment, $t_{i}$. We assume that if the administrator is indifferent between an equilibrium treatment and one recommended by a credible neologism, then she keeps to the putative equilibrium:

$$
\begin{aligned}
& \theta_{1} \text { solves }\left(1+\theta_{1}\right)=\frac{E\left[\pi \mid Z_{2}\right]}{\gamma_{12}} \\
& \theta_{2} \text { solves }\left(1+\theta_{2}\right)=\frac{E\left[\pi \mid Z_{2} \cup Z_{3}\right]}{\gamma_{12}} \\
& \theta_{3} \text { solves }\left(1+\theta_{3}\right)=\frac{E\left[\pi \mid Z_{23} \cup Z_{3}\right]}{\gamma_{23}} \\
& \theta_{4} \text { solves }\left(1+\theta_{4}\right)=\frac{E\left[\pi \mid Z_{3}\right]}{\gamma_{23}}
\end{aligned}
$$


Let $\underline{\theta}=\min \left\{\theta_{1}, \theta_{4}\right\}$ and $\bar{\theta}=\max \left\{\theta_{2}, \theta_{3}\right\}$. As $\theta_{1}<\theta_{2}$ and $\theta_{3}<\theta_{4}$, this implies that $\underline{\theta}<\bar{\theta}$.

Proofs of the following three propositions are given in Appendix A.3.1.

Proposition 1 For $\underline{\theta}$ and $\bar{\theta}$ as defined above

i) A NPE with menu of treatments $T_{0}=\left\{t_{1}, t_{2}, t_{3}\right\}$ exists if and only if $\theta \leq \underline{\theta}$.

ii) A NPE with menu of treatments $T_{0}=\left\{t_{1}\right\}$ exists if and only if $\bar{\theta} \leq \theta$.

The two parts in proposition (1) reflect the degree of alignment between the players' preferences and the level of meaningful communication between them. The closer the alignment, the greater the amount of meaningful communication. For example, the administrator will authorise an alternative treatment to $t_{1}$ if the marginal cost of increasing the treatment is less than her expected marginal benefit, that is, the reduction in expected harm. The conditions for this proposition are satisfied if and only if the necessary conditions for the respective outcomes hold, as given in Table 4.6, and a greater range of treatments tend to be authorised with lower values of $\theta$ (we give a specific numerical example below). This proposition ensures that an equilibrium outcome exists for $\theta \leq \underline{\theta}$ and $\bar{\theta} \leq \theta$.

Proposition 2 For $\underline{\theta}$ and $\bar{\theta}$ as defined above,

i) A NPE with the menu of treatments $T_{0}=\left\{t_{1}, t_{2}\right\}$ exists if and only if $\theta_{4} \leq \theta \leq \theta_{2}$.

ii) A NPE with the menu of treatments $T_{0}=\left\{t_{1}, t_{3}\right\}$ exists if and only if $\theta_{1} \leq \theta \leq \theta_{3}$.

Following a similar intuition as above, an NPE outcome exists with at least one of the menus $T_{0}=\left\{t_{1}, t_{2}\right\}$ or $T_{0}=\left\{t_{1}, t_{3}\right\}$ if no credible neologism exists for the omitted treatment and the interval $[\underline{\theta}, \bar{\theta}]$ is non-empty. However, one of these NPE outcomes does not necessarily exist for the entire interval $[\underline{\theta}, \bar{\theta}]$.

Because $\theta_{3}<\theta_{4}$, we can draw some conclusions about the existence and uniqueness of the NPE outcomes. First, there can be no overlap between the two types of equilibria described by Proposition 1. This conclusion, taken together with Proposition 2, implies 
that an equilibrium outcome is unique, except when an equilibrium outcome does not exist for some $\theta \in\{\underline{\theta}, \bar{\theta}\}$.

Furthermore, the equilibrium correspondence on $\theta$ can take three different base shapes:

1. The correspondence might include an equilibrium outcome with $T_{0}=\left\{t_{1}, t_{2}\right\}$ over the entire range $[\underline{\theta}, \bar{\theta}]$.

2. The correspondence might include an equilibrium outcome with $T_{0}=\left\{t_{1}, t_{3}\right\}$ over the entire range $[\underline{\theta}, \bar{\theta}]$.

3. There might be a region in which there is non-existence.

In the third case, this region of non-existence might encompass the entire range $(\underline{\theta}, \bar{\theta})$. However, there might be a region with $T_{0}=\left\{t_{1}, t_{3}\right\}$ between $\underline{\theta}$ and the region of nonexistence. Likewise, there might be a region with $T_{0}=\left\{t_{1}, t_{2}\right\}$ between the region of nonexistence and $\bar{\theta}$. Proposition 3 in Appendix A.3.1 explicitly examines these possibilities. Together the three propositions guarantee that any of the four NPE outcomes may exist, and a NPE may exist for the entire range of $\theta$, but not necessarily. Below we provide an example illustrating these propositions.

This analysis shows that an outcome with less intensive or more intensive treatment could occur. Thus, CHE reformers might have expected costs to fall, but it is possible to move to an outcome that resulted in higher costs. In section 4.4.5 we examine how sharpening the administrator's incentives can result in various outcomes and the impact on expected treatment costs and patient harm. 


\section{Example of NPE outcome existence for the range of $\theta$}

Consider the following illustrative example where $\pi \sim U[0,1]$ and given the parameters in Table 4.7 on page 103 . In this example, $\gamma_{12}=0.17, \gamma_{13}=0.2, \gamma_{23}=0.22$, which implies that $\theta_{1}=1.15, \theta_{2}=3.5, \theta_{3}=2.76$, and $\theta_{4}=2.8$.

\begin{tabular}{|c|c|c|}
\hline Treatment $\left(t_{i}\right)$ & $C\left(t_{i}\right)$ & $\delta\left(t_{i}\right)$ \\
\hline 1 & 3.7 & 0 \\
\hline 2 & 2.5 & 0.2 \\
\hline 3 & 0.2 & 0.7 \\
\hline
\end{tabular}

Table 4.7: Illustrative example of NPE outcome existence

This example captures the richest case where any of the four of the NPE outcomes may exist (see Appendix A.3.1). It might also be that any one of the three zones in the range $(\underline{\theta}, \bar{\theta})$ might expand, so only one or neither of the intermediate outcomes might exist.

Figure 4.2 on page 103 uses the numerical thresholds above. The figure shows how as $\theta$ rises, the degree of communication falls. It indicates that any of the four equilibrium outcomes can exist for $0 \leq \theta$, but there need not be a NPE if $\theta$ is between $\theta_{3}$ and $\theta_{4}$.

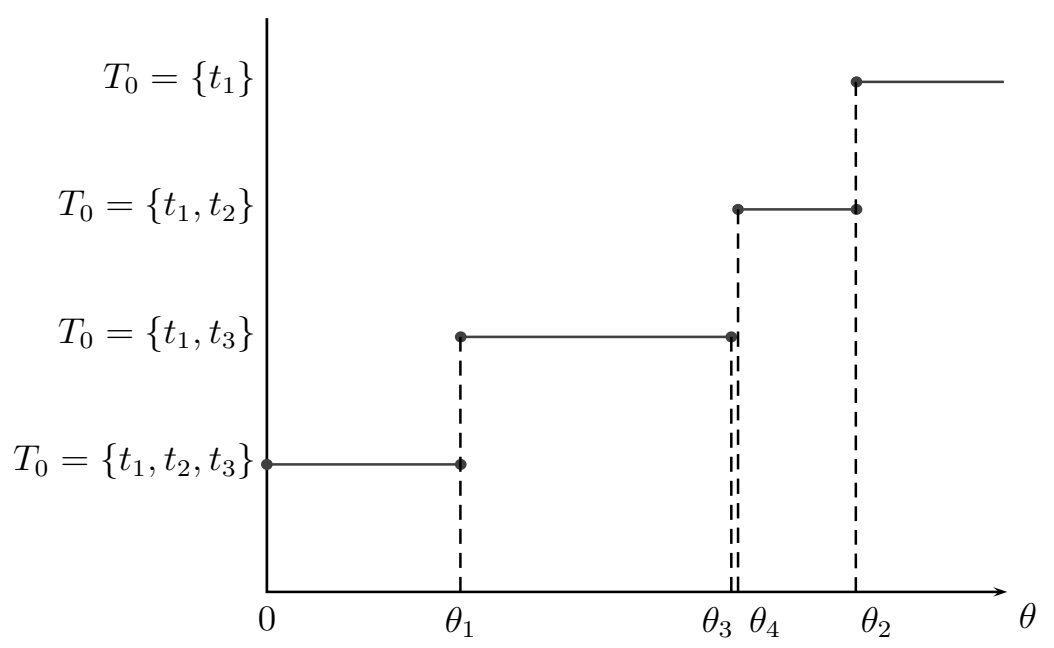

Figure 4.2: Example of equilibrium outcomes with various $T_{0}$ for a range of $\theta$ 


\subsubsection{Sharp incentives}

This section considers how sharpening the administrator's incentives might change treatment costs and expected harm. We analyse how these costs change with a move from a base case to one of the other NPE outcomes characterised above.

We assume that the funder could use the administrator's contract to sharpen the administrator's incentives for cost control. This affects the degree of misalignment between the specialist's and the administrator's preferences.

In practice, the funder will have some uncertainty when setting the administrator's contract. In the CHE reforms, there were many treatment options. The effect of sharpening incentives may have been clearer and more effective for some treatments or medical specialities than for others. As such, the funder might not be able to determine precisely which outcome would occur as a result of sharpening the administrator's incentives. Therefore, we explore the range of possibilities. In one case, sharper incentives counterproductively cause an increase in expected treatment costs as well as expected harm.

The funder's expected utility depends on both treatment costs and harm, that is, $-[(1+\lambda) C(t)+\pi \delta(t)]$, where $\lambda>0$. For ease of exposition, we refer to this expression as 'total costs', from the perspective of the funder. Here, the funder puts a greater weight on treatment costs than on the harm of an adverse event in evaluating expected total cost.

We assume that the funder can frame the administrator's contract in order to set $\theta=\lambda$. This would appear to align the administrator's interests with the funder's interests ${ }^{31}$ We consider $\theta=0$ or $\theta=\lambda>0$, and examine whether the impacts of sharpening the administrator's incentives by increasing $\theta$ to $\lambda$ can reduce the funder's expected payoff. ${ }^{32}$

\footnotetext{
${ }^{31}$ In this model, the funder cannot incentivise the specialist equally, as the specialist's actions relate to non-verifiable information. The assumption of a weaker ability to incentivise a specialist is plausible for doctors in the New Zealand health sector, and was discussed in section 4.2 .

${ }^{32}$ Appendices A.3.1 and A.3.2 give a proof of the existence of the four NPE outcomes in Table 4.6 (on page 99p and illustrative examples of when the NPE outcomes may occur for various parameter values. The examples show that it is possible to move from one outcome to one of several others by sharpening the administrator's incentives. If the funder is uncertain about some parameters, sharpening the administrator's incentives may increase or reduce the funder's expected utility. We discuss the implication of this below, including in footnote 36 .
} 
If $\theta=0$, then the administrator's and specialist's interests are aligned. We show an NPE outcome with a treatment menu of $T_{0}=\left\{t_{1}, t_{2}, t_{3}\right\}$ for this case at the end of section 4.4.4. If $\theta=\lambda>0$, then in equilibrium, $T_{0}$ could be any of $\left\{t_{1}, t_{2}, t_{3}\right\},\left\{t_{1}, t_{2}\right\},\left\{t_{1}\right\}$ or $\left\{t_{1}, t_{3}\right\}$ from Table 4.6. The equilibrium outcome depends how the misalignment of preferences affects meaningful communication between the specialist and administrator. Sharpening the administrator's incentives, $\theta>0$, does not affect the expected utility of the funder if it still results in the outcome with the full treatment menu, $T_{0}=\left\{t_{1}, t_{2}, t_{3}\right\}$.

Below, we consider the remaining three cases in Table 4.6. We compare the expected treatment and total costs from the funder's perspective in these three cases to the base case, $T_{0}=\left\{t_{1}, t_{2}, t_{3}\right\}$.

The first case is when an increase in $\theta$ results in an outcome with $T_{0}=\left\{t_{1}, t_{2}\right\}$. In this case, the authorised treatment would be the same as in the base case unless $\pi$ is greater than $\gamma_{23}$. In this upper range, treatment costs would only be $C\left(t_{2}\right)$ rather than $C\left(t_{3}\right)$. Thus, we can focus on when $\gamma_{23}<\pi$ to assess the change in expected total costs. Expected total costs would fall, relative to $T_{0}=\left\{t_{1}, t_{2}, t_{3}\right\}$, if the following condition is satisfied 33

$$
E\left[\pi \mid \gamma_{23} \leq \pi\right]<(1+\lambda) \gamma_{23}
$$

\footnotetext{
${ }^{33}$ Expected total costs will fall in this case if the following condition is true:$$
\int_{0}^{\gamma_{12}}\left((1+\lambda) C\left(t_{1}\right)+\pi \delta\left(t_{1}\right)\right) d H+\int_{\gamma_{12}}^{1}\left((1+\lambda) C\left(t_{2}\right)+\pi \delta\left(t_{2}\right)\right) d H
$$$$
<\int_{0}^{\gamma_{12}}\left((1+\lambda) C\left(t_{1}\right)+\pi \delta\left(t_{1}\right)\right) d H+\int_{\gamma_{12}}^{\gamma_{23}}\left((1+\lambda) C\left(t_{2}\right)+\pi \delta\left(t_{2}\right)\right) d H+\int_{\gamma_{23}}^{1}\left((1+\lambda) C\left(t_{3}\right)+\pi \delta\left(t_{3}\right)\right) d H
$$$$
\Rightarrow \quad \int_{\gamma_{23}}^{1}\left((1+\lambda) C\left(t_{2}\right)+\pi \delta\left(t_{2}\right)\right) d H<\int_{\gamma_{23}}^{1}\left((1+\lambda) C\left(t_{3}\right)+\pi \delta\left(t_{3}\right)\right) d H
$$$$
\Rightarrow P\left(\gamma_{23} \leq \pi\right)\left[(1+\lambda) C\left(t_{2}\right)+E\left[\pi \mid \gamma_{23} \leq \pi\right] \delta\left(t_{2}\right)\right]<P\left(\gamma_{23} \leq \pi\right)\left[(1+\lambda) C\left(t_{3}\right)+E\left[\pi \mid \gamma_{23} \leq \pi\right] \delta\left(t_{3}\right)\right]
$$$$
\Rightarrow E\left[\pi \mid \gamma_{23} \leq \pi\right]<(1+\lambda) \gamma_{23} \text {. }
$$ 
When $\theta=\lambda$ condition 4.18 must hold for $T_{0}=\left\{t_{1}, t_{2}\right\}$ to be consistent with an NPE outcome, as shown in Table 4.6. That is, in this outcome the funder judges that, on average, $t_{2}$ is best when $\gamma_{23}<\pi$, and expects total costs to fall. Therefore, an increase in $\theta$ to $\lambda$ would be beneficial from the funder's perspective as it lowers both treatment costs and expected total costs.

A second case is when an increase in $\theta$ results in an outcome with $T_{0}=\left\{t_{1}\right\}$. Although expected treatment costs would also fall, unlike the first case, expected total costs might rise or fall. Expected treatment costs would fall as treatment costs would be $C\left(t_{1}\right)$ regardless of the patient's condition $\pi$. Any patient who would have received $t_{2}$ or $t_{3}$ would receive $t_{1}$ in this outcome.

Expected total costs, however, would rise if the decrease in treatment costs was less than the increase in expected harm. Expected harm would increase relative to the base case if a patient were to receive a lower intensity treatment, that is, $t_{1}$ rather than $t_{2}$ or $t_{3}$. Thus, expected total costs would increase with a move to $T_{0}=\left\{t_{1}\right\}$ if the following condition is satisfied:

$$
\begin{aligned}
\int_{\gamma_{12}}^{\gamma_{23}} & \left((1+\lambda) C\left(t_{2}\right)+\pi \delta\left(t_{2}\right)\right) d H+\int_{\gamma_{23}}^{1}\left((1+\lambda) C\left(t_{3}\right)+\pi \delta\left(t_{3}\right)\right) d H \\
< & \int_{\gamma_{12}}^{1}\left((1+\lambda) C\left(t_{1}\right)+\pi \delta\left(t_{1}\right)\right) d H .
\end{aligned}
$$

This may be expressed as:

$$
\begin{aligned}
& P\left(\gamma_{23} \leq \pi\right)\left(\delta\left(t_{1}\right)-\delta\left(t_{3}\right)\right)\left((1+\lambda) \gamma_{13}-E\left[\pi \mid \gamma_{23} \leq \pi\right]\right) \\
& \quad<P\left(\gamma_{12} \leq \pi \leq \gamma_{23}\right)\left(\delta\left(t_{1}\right)-\delta\left(t_{2}\right)\right)\left(E\left[\pi \mid \gamma_{12} \leq \pi \leq \gamma_{23}\right]-(1+\lambda) \gamma_{12}\right)
\end{aligned}
$$

For an NPE with $T_{0}=\left\{t_{1}\right\}$, when $\theta=\lambda$, the right hand side of equation (4.19) is nonpositive according to the conditions in Table $4.6{ }^{34}$ That is, a necessary condition for a

\footnotetext{
${ }^{34}$ Condition 14, marked $D 1$, in Table 4.6 requires $E\left[\pi \mid \pi \in Z_{2} \cup Z_{3}\right] \leq(1+\theta) \gamma_{12}$. Satisfying this condition also implies that $E\left[\pi \mid \pi \in Z_{2}\right] \leq(1+\theta) \gamma_{12}$. Thus, when $\theta$ is set to $\lambda$, this latter condition implies that $E\left[\pi \mid \gamma_{12} \leq \pi \leq \gamma_{23}\right] \leq(1+\lambda) \gamma_{12}$.
} 
switch to $T_{0}=\left\{t_{1}\right\}$ in equilibrium is that the funder expects total costs to fall for patients when $\pi$ is between $\gamma_{12}$ and $\gamma_{23}$. The left hand side, however, could be either positive or negative. Thus, expected total costs could rise or fall when $\pi$ is above $\gamma_{23}$.

For example, if the probability that $\pi$ is between $\gamma_{12}$ and $\gamma_{23}$ is small enough, then the switch to $T_{0}=\left\{t_{1}\right\}$ will increase expected total costs from the funder's perspective if $(1+\lambda) \gamma_{13}<E\left[\pi \mid \gamma_{23} \leq \pi\right]$. This might be the case where there is only a relatively small group of moderately ill patients $\left(\pi \in Z_{2}\right)$, and the remainder require either minor $\left(\pi \in Z_{1}\right)$ or intensive $\left(\pi \in Z_{3}\right)$ treatment. The increase in expected harm for the latter group could be greater than the saving in their treatment costs from receiving $t_{1}$ rather than $t_{3}$. In this case, sharper incentives would be counterproductive.

A third case is when an increase in $\theta$ results in the outcome with $T_{0}=\left\{t_{1}, t_{3}\right\}$. Unlike the two cases above, both expected treatment costs and expected total costs may rise. In this outcome, patients receiving $t_{2}$ would be reallocated to either $t_{1}$ or $t_{3}$. Expected treatment costs would rise if the following condition is satisfied 35

$$
\int_{\gamma_{12}}^{\gamma_{23}}(1+\lambda) C\left(t_{2}\right) d H<\int_{\gamma_{12}}^{\gamma_{13}}(1+\lambda) C\left(t_{1}\right) d H+\int_{\gamma_{13}}^{\gamma_{23}}(1+\lambda) C\left(t_{3}\right) d H
$$

This may be expressed as:

$$
P\left(\gamma_{12} \leq \pi \leq \gamma_{13}\right)\left(C\left(t_{2}\right)-C\left(t_{1}\right)\right)<P\left(\gamma_{13} \leq \pi \leq \gamma_{23}\right)\left(C\left(t_{3}\right)-C\left(t_{2}\right)\right)
$$

Expected treatment costs could rise or fall with a move to this outcome. The change depends on the distribution of $\pi$, and therefore on the appropriateness of reallocating

${ }^{35}$ This condition is a simplification of:

$$
\begin{aligned}
& \int_{0}^{\gamma_{12}}(1+\lambda) C\left(t_{1}\right) d H+\int_{\gamma_{12}}^{\gamma_{23}}(1+\lambda) C\left(t_{2}\right) d H+\int_{\gamma_{23}}^{1}(1+\lambda) C\left(t_{3}\right) d H \\
& \quad<\int_{0}^{\gamma_{13}}(1+\lambda) C\left(t_{1}\right) d H+\int_{\gamma_{13}}^{1}(1+\lambda) C\left(t_{3}\right) d H .
\end{aligned}
$$


patients who would have received $t_{2}$ to either $t_{1}$ or $t_{3}{ }^{36}$ When $\pi \in Z_{12}$ a patient would receive the less expensive treatment of $t_{1}$ rather than $t_{2}$; but when $\pi \in Z_{23}$ a patient would receive $t_{3}$ rather than $t_{2}$. The increase in treatment costs from patients ratcheted up from $t_{2}$ to $t_{3}$ may be greater than the savings from those $t_{2}$ patients reallocated to $t_{1}$. For example, condition 4.20 would be satisfied when the probability that $\pi$ is between $\gamma_{12}$ and $\gamma_{13}$ is relatively low, and the probability that $\pi$ is between $\gamma_{13}$ and $\gamma_{23}$ is relatively high.

Expected total costs would rise if the following condition is satisfied:

$$
\begin{aligned}
\int_{\gamma_{12}}^{\gamma_{23}} & \left((1+\lambda) C\left(t_{2}\right)+\pi \delta\left(t_{2}\right)\right) d H< \\
& \int_{\gamma_{12}}^{\gamma_{13}}\left((1+\lambda) C\left(t_{1}\right)+\pi \delta\left(t_{1}\right)\right) d H+\int_{\gamma_{13}}^{\gamma_{23}}\left((1+\lambda) C\left(t_{3}\right)+\pi \delta\left(t_{3}\right)\right) d H
\end{aligned}
$$

This may be expressed as:

$$
\begin{gathered}
P\left(\gamma_{12} \leq \pi \leq \gamma_{13}\right)\left(\delta\left(t_{1}\right)-\delta\left(t_{2}\right)\right)\left((1+\lambda) \gamma_{12}-E\left[\pi \mid \gamma_{12} \leq \pi \leq \gamma_{13}\right]\right)< \\
P\left(\gamma_{13} \leq \pi \leq \gamma_{23}\right)\left(\delta\left(t_{2}\right)-\delta\left(t_{3}\right)\right)\left((1+\lambda) \gamma_{23}-E\left[\pi \mid \gamma_{13} \leq \pi \leq \gamma_{23}\right]\right)
\end{gathered}
$$

If $T_{0}=\left\{t_{1}, t_{3}\right\}$ is consistent with an NPE outcome, when $\theta=\lambda$ the left hand side of equation (4.21) is non-negative by condition 5 in Table $4.6{ }^{37}$ This reflects that, in this outcome, the funder does not expect total costs to rise for the group reallocated from $t_{2}$ to $t_{1}$ (when $\pi \in Z_{12}$ ). The right hand side is necessarily positive, as $E\left[\pi \mid \pi \in Z_{23}\right]<(1+\lambda) \gamma_{23}$ for $0<\lambda$. Therefore, this inequality does not necessarily hold, so expected total costs may rise or fall with a change to this outcome ${ }^{38}$ Thus, the additional harm to patients

\footnotetext{
${ }^{36}$ When $T_{0}=\left\{t_{1}, t_{3}\right\}$, the specialist cannot convince the administrator to authorise $t_{2}$. As such, he must compromise when $\gamma_{12} \leq \pi \leq \gamma_{23}$ even though he would prefer the patient to receive $t_{2}$. Instead, the specialist has to make a less than ideal recommendation (from his perspective): $t_{1}$ when $\pi \in Z_{12}$, i.e. $t_{21}$, and $t_{3}$ when $\pi \in Z_{23}$, i.e. $t_{23}$.

${ }^{37}$ Condition 5 in Table 4.6 requires $E\left[\pi \mid \pi \in Z_{2}\right] \leq(1+\theta) \gamma_{12}$. Satisfying this condition then implies that $E\left[\pi \mid \pi \in Z_{12}\right] \leq(1+\theta) \gamma_{12}$.

${ }^{38} \mathrm{~A}$ move to this outcome would see a patient who would have received $t_{2}$ receive $t_{3}$ when $\gamma_{13} \leq \pi \leq$ $(1+\lambda) \gamma_{23}$, which is a higher treatment level than the funder prefers, but inappropriately receive $t_{1}$ when $(1+\lambda) \gamma_{12} \leq \pi \leq \gamma_{13}$. The reduction in expected total costs from patients appropriately receiving less intensive treatment need not offset the increases from the inappropriate reallocations.
} 
reallocated from $t_{2}$ to $t_{1}$ plus the extra treatment cost of patients reallocated from $t_{2}$ to $t_{3}$ may outweigh the cost savings from the restricting treatment menu and reallocating some patients from $t_{2}$ to $t_{1}$.

The insight from section 4.4.1 was not just that high powered incentives could be harmful by increasing expected harm. Rather, sharper incentives to control costs can counterproductively cause an increase in expected treatment costs. The illustration of section 4.4 .1 corresponds to an outcome with $T_{0}=\left\{t_{1}, t_{3}\right\}$ in which $\pi$ is never less than $\gamma_{13}$. In this special case, the specialist would never want to recommend $t_{1}$. As a result, the impact on treatment and total costs with $T_{0}=\left\{t_{1}, t_{3}\right\}$ is equivalent to an outcome with $T_{0}=\left\{t_{3}\right\}$. This implies that equation (4.21) is satisfied when $E\left[\pi \mid \gamma_{13} \leq \pi \leq \gamma_{23}\right]<(1+\lambda) \gamma_{23}$, and this condition is true for any $0<\lambda$, as $E\left[\pi \mid \pi \in Z_{23}\right] \leq \gamma_{23}$. This case is consistent with the corresponding NPE outcome in Table 4.6 when $\theta=\lambda$ if $E\left[\pi \mid \pi \in Z_{23}\right]<(1+\lambda) \gamma_{23}<$ $E\left[\pi \mid \pi \in Z_{23} \cup Z_{3}\right]$. Under these conditions, a contract that lifts $\theta$ and results in an NPE outcome of $T_{0}=\left\{t_{1}, t_{3}\right\}$ would increase treatment costs as well as total costs.

In the case of the CHE reforms, the reforms may have expected a reduction in treatment and total costs as predicted by case 1, or may have predicted a reduction treatment costs even if total costs were to rise as in cases 2 or 3 . However, case 3 shows that dividing the administrator's and specialist's incentives may not only increase expected total costs; it could also increase expected treatment costs. This theoretical result may help to provide a more sophisticated understanding of why, despite giving CEOs strong incentives for cost control, many hospitals' deficits escalated contrary to expectations, thereby "frustrating attempts to institute equitable, consistent and agreed rationing" (Crown Company Monitoring Advisory Unit 1996, 8). 


\subsection{Discussion}

This chapter shows how stronger incentives for cost control can perversely raise an organisation's costs. Sharper managerial incentives for cost control can reduce the credibility of an informed specialist with an administrator who is responsible for authorising treatment. This loss of credibility can reduce meaningful communication between the two actors, resulting in greater expected harm and potentially increased costs.

This result is novel and contrasts with the standard 'quality-quantity' trade-off. We develop a multi-layered organisational hierarchy to model how sharp incentives affect the communication of soft information. We show how increasing incentives for a verifiable

dimension of output, such as increasing cost control, can affect patient harm (quality) and treatment costs (quantity). Sharper incentives increase expected patient harm and counterproductively worsen expected treatment costs.

The model also highlights that stronger incentives may have an uncertain effect, as costs may increase or decrease depending on the outcome that results. For some parameters, an equilibrium may not exist, so it is not possible to determine precisely what outcome would occur as a result of strengthening incentives. This suggests that the reformers might have been unable to confidently determine ex ante which outcome would occur, but ex post might have wished to reverse the reforms in light of an undesirable outcome.

The CHE reformers may have incorrectly estimated the risk profile of the population. In this case reformers may have believed that pursuing the reforms would reduce treatment cost and improve efficiency. Instead, sharp managerial incentives may have contributed to inflating costs and flagging service quality. 


\section{Chapter 5}

\section{Advocacy and conflicts of interest}

\section{$5.1 \quad$ Introduction}

The CHE reforms created public concern that cost-orientated administrators were less interested in patients' health than were the previous hospital management teams (Crown Company Monitoring Advisory Unit 1997, 6). Chapter 4 showed how sharp incentives

for cost control could worsen patient outcomes in spite of medical specialists' efforts to communicate with managers.

The public's concern may have given medical specialists an expanded role to act as patient advocates. But this expanded role might also have given specialists greater power to pursue their own private interests. In this chapter, we address a third criticism of the CHE reforms: that the reforms gave medical specialists the opportunity and incentives to manipulate managers politically, which reduced hospital efficiency.

This criticism hinges on when a consumer should believe a specialist. We add a further layer to the organisational hierarchy outlined in Chapter 4 by introducing a consumer. We model a consumer's response to a specialist indicating that service quality is poor. If the consumer believes the specialist, she would hold the administrator accountable and support the dismissal of the administrator. But the information the specialist communicates is "soft" (Aghion \& Tirole 1997, 7), and the consumer knows the specialist is torn 
by conflicting incentives. Although the specialist has an ethical responsibility to act in a patient's best interest, he also has a private interest in his own working conditions, which are determined by the administrator.

We develop two theoretical models to answer the following questions:

- Can an informed specialist use quality warnings to manipulate the public to his advantage?

- Might an administrator benefit from allowing a specialist to communicate information on quality?

- Can an administrator benefit from reducing communication by using her own efforts and her authority in the organisation?

We use an innovative approach by embedding cheap talk subgames and using neologismproofness to identify plausible equilibria. We reinforce our finding of Chapter 4 that a principal may face a tradeoff between sharp incentives for an administrator's performance and soft incentives that promote communication. In this chapter, the communication is between a specialist and a consumer.

Section 5.3 sets up a three-actor model involving a consumer, an administrator, and a specialist, who reports on service quality to the consumer. We focus on when the administrator wants the specialist to send a credible signal to the consumer.

We modify the first model in section 5.4 to explore an important feature of the CHE reforms: the effort of CEOs to reform the systems and incentives within hospitals. We assume that the administrator puts in effort that increases the probability of high quality outcomes. This reduces the administrator's dependence on the specialist to provide a credible quality signal.

A result of both models is that the specialist can sometimes benefit from his role as an agent for patients. However, the modified model also shows that the administrator might take advantage of her role in providing effort and controlling the specialist's credibility. 
Although the first result is consistent with the third criticism of the reforms, we find that medical specialists would not necessarily have been able to manipulate the public. Moreover, the second result suggests that CHE managers might have benefitted from their efforts, but such efforts may have resulted in inefficiently low communication.

\subsection{Background to the patient-doctor relationship in the CHE reforms}

The health sector reforms that introduced CHEs also reduced the public's direct influence over governance. Easton $(1997,154)$ concluded that the abolition of "the (two thirds)

elected [area health] boards... eras[ed] overnight the one institutionalised and well-funded group with official moral authority who could have resisted the reforms". He argues that reform initiatives, such as the introduction of user charges, "warned [the public] that the reform proposals were radical and - in its judgement - nasty" (Easton 1997, 156).

The quote above suggests some public suspicion of the CHE reforms. The cost orientation of the newly installed CHE managers may have reduced the public's trust that hospital managers were working in patients' best interests. This created a public perception that there was a gap in accountability for patients' health interests under the new system (Crown Company Monitoring Advisory Unit 1997, 7).

Howell (2000) suggests that doctors observed the gap in the management structure created by public distrust, and sought to take up the role of patients' advocates in order to "gain some control of the executive process". That is, doctors took advantage of the opportunities presented by the reforms to manipulate the system. Howell $(2000,6)$ concludes that the reforms meant doctors "increased their effective power as it was unchecked by any formal accountability process. Opportunistic exploitation of this unbridled power was inevitable, with consequent efficiency losses...".

In several instances during the reforms, medical professionals publicly criticised managerial proposals and in some cases prevented managers from exercising their delegated 
authority (Vaithianathan 1999b). Anecdotal evidence suggests that some CHE managers lost control of some aspects of the hospital's management (George et al. 1997). Over half of the CEOs resigned within three years of appointment, many citing frustration at not being able to carry out their plans, with one noting the difficulty of "meet[ing] the conflicting expectations of the public, staff, and the Government" (Gilling 1996, 6). Vaithianathan $(1999 b, 176-77)$ cautions that surgeons had financial incentives to manipulate the supply of public hospital services, to "ensure a steady supply of patients for their private practices". We focus on a different mechanism: the manipulation of a patient's beliefs about service quality, rather than a dynamic related to hospital waiting lists.

In addition to the literature on the CHE reforms, we draw on ideas developed by Tirole (1986) and Aghion \& Tirole (1997) on communication in hierarchies. We add to this theoretical literature by formally modelling the communication of "soft" information in a four-layer organisational hierarchy. Our model also endogenises the administrator's choices of effort and the degree of information transfer.

\subsection{Conflicts of interest and credibility}

We present a three-actor model involving a consumer, an administrator, and a specialist to address our first two research questions in this chapter. We set up the game in section 5.3.1. In section 5.3.2 we characterise the potential Perfect Bayesian Nash equilibria (PBE) of the game. We initially focus on the subgames of these PBE outcomes. We examine the specialist's incentives to communicate truthfully and how the consumer should respond in these subgames.

One drawback with the PBE concept is that implausible equilibria are possible. For example, in a babbling equilibrium the specialist does not expect his message to be interpreted as meaningful, so he is willing to send any conceivable message, that is, to babble. Such an outcome is implausible if meaningful communication is in the interests of both the sender and receiver. 
Therefore, in section 5.3.3, we use the equilibrium refinement of neologism-proofness, which rules out these implausible equilibria. We outline the circumstances under which the administrator is willing to pay for the specialist to communicate credibly, and characterise a set of conditions for a plausible PBE outcome with neologism-proof subgames (an NPE).

\subsubsection{Play of the game}

The model has three players: an administrator, $A$, a specialist, $S$, and a consumer, $C$.

In general, an administrator could offer a specialist a package of remuneration and working conditions that includes both pecuniary and non-pecuniary elements. We represent this general possibility with a simple, concrete version by assuming that the administrator offers the specialist a wage.

The steps of the game are as follows:

1. A chooses a wage to offer $S, w \in\left[0, w_{G}\right]$.

2. $S$ observes $w$ and decides whether to accept the offer, $O$. If he rejects it, $Q$, then the player's receive their reservation utilities, $\bar{u}_{i}, i \in\{A, S, C\}$. Otherwise ...

3. Nature, $N$, chooses the organisation's quality level, $q \in\left\{q_{L}, q_{H}\right\}$, where $P\left(q=q_{H}\right)=\pi$.

4. $S$ observes $q$ (as well as $w$ ), and chooses a message, $m \in M$.

5. $C$ observes $w$ and $m$, but not $q$, before making her decision. $C$ decides whether to reappoint $A$ or terminate, $y \in\{R, T\}$.

Figure 5.1 on page 116 illustrates the steps of the game; the utility functions of the players are specified in array 5.1 on page 117 . 


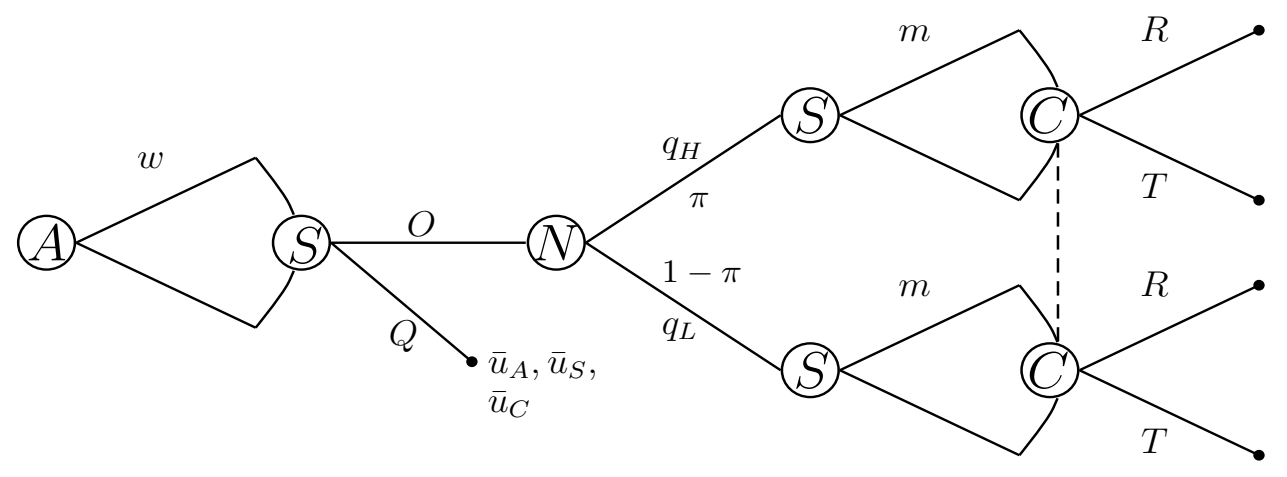

Figure 5.1: Game tree for three-actor model

We assume that the administrator is initially uncertain about the quality level, but must commit to a wage for the specialist before she observes quality. The specialist has conflicting incentives: he is not a perfect agent for the consumer and he may not always want to reveal quality accurately. The administrator may use the specialist's wage to support or erode his credibility, essentially turning a quality signal to the consumer on or off.

The specialist's participation decision will be characterised by a reservation wage, $w_{B}$. His reservation wage will depend on the value of his outside option, $\bar{u}_{S}$, and on $\pi$, as this affects the anticipated equilibrium and thus the specialist's payoff in the second stage. Below, we treat $w_{B}$ as a constant, given $\pi$, but do not assume a specific value $!^{1}$

If the specialist accepts the offer, he observes quality and decides what message about quality to send. We treat the specialist's message as cheap talk. ${ }^{2}$ Such a message is comprehensible to the consumer but is not necessarily credible. Let $M_{i}$ be a set of messages with the literal meaning indicating that quality is $q_{i}$.

We model the consumer's ability to hold an administrator accountable for service quality as a decision of whether to reappoint or dismiss the administrator. This step is a placeholder for some political process. For example, the public may vote on the funder's tenure,

\footnotetext{
${ }^{1}$ In Appendix A.4.2 we derive expressions for the reservation wage for different values of $\bar{u}_{S}$ and $\pi$.

${ }^{2}$ This assumption may be interpreted as medical specialists having no formal authority to report on quality.
} 
whose fate also affects the administrator ${ }^{3}$ This allows the public to hold the funder, and thereby the administrator, responsible for quality.

We use the following terminology in the players' utility functions, which are given below:

- An administrator's benefit from her tenure is $G$.

- The specialist cares about his wage, $w$, and quality, $q$.

- The consumer expects quality of $q^{\prime}$ under a replacement administrator ${ }^{4}$

- The specialist's expected utility under a new administrator's regime is $w^{\prime}+q^{\prime}$.

$$
\begin{aligned}
& U_{A}=\left\{\begin{array}{l}
2(G-w) \text { if reappointed } \\
G-w \text { otherwise }
\end{array}\right. \\
& U_{S}=\left\{\begin{array}{l}
2(w+q) \text { if } C \text { reappoints } A \\
w+q+w^{\prime}+q^{\prime} \text { otherwise }
\end{array}\right. \\
& U_{C}=\left\{\begin{array}{l}
2 q \text { if } C \text { reappoints } A \\
q+q^{\prime} \text { otherwise }
\end{array}\right.
\end{aligned}
$$

If the administrator is reappointed and has two periods of tenure, for example, then the quality level is the same in both periods, and the consumer receives $2 q$. If the administrator is dismissed, expected quality in the second period is $q^{\prime}$, so she receives $q+q^{\prime}$.

\footnotetext{
${ }^{3}$ The political process could be thought of as a simplified version of New Zealand's democratic voting system. Although a CHE manager was not an elected position, the role, and to some degree the appointee, depends on the health system implemented by the elected politicians. When the public votes for change, then the system and its senior actors, such as managers, may be removed as the system is reformed.

${ }^{4} \mathrm{We}$ do not assume that the quality provided by the incumbent administrator is equal to that expected under a replacement administrator. Thus, when the specialist's message is not credible and the consumer is imperfectly informed, the expected value of $q$ need not equal that expected under a replacement administrator, $q^{\prime}$.
} 


\subsubsection{Why setting wages can turn signals on and off}

We use backwards induction to identify potential PBE outcomes for this game.

The array below outlines the conditions under which each player would want a particular tenure decision if $q$ were known. Let $Y=\{R, T\}$ be the set of possible tenure decisions:

$$
\begin{aligned}
& y_{A}^{\star}=\left\{\begin{array}{lll}
T & \text { if } & G<w \\
R & \text { if } & 0 \leq G-w
\end{array}\right. \\
& y_{S}^{\star}=\left\{\begin{array}{lll}
T & \text { if } & w+q<w^{\prime}+q^{\prime} \\
R & \text { if } & w^{\prime}+q^{\prime} \leq w+q
\end{array}\right. \\
& y_{C}^{\star}=\left\{\begin{array}{lll}
T & \text { if } & q<q^{\prime} \\
R & \text { if } & q^{\prime} \leq q .
\end{array}\right.
\end{aligned}
$$

\section{The consumer's choice}

We assume that the consumer prefers the administrator to be replaced if she believes quality is low, and to be reappointed if she believes quality is high. That is, we assume:

$$
q_{L}<q^{\prime}<q_{H} .
$$

At step 5 in Figure 5.1, the consumer's choice depends on her beliefs about whether quality is high under the incumbent administrator. If the consumer learns the quality level through a credible message from the specialist then she acts accordingly.

If the consumer does not learn the quality level, she acts on her prior beliefs about quality. The consumer will reappoint when she is uninformed if her expected payoff is greater than her expected utility under a replacement administrator. The consumer's best choice is to reappoint when uninformed if she believes $\pi$ is equal to or above some threshold, 
$\pi^{*}=\frac{q^{\prime}-q_{L}}{q_{H}-q_{L}}$. We describe this case as the consumer being optimistic $5^{5}$ Equivalently, we describe the consumer as pessimistic when $\pi<\pi^{*}$ and she would prefer to dismiss the administrator if uninformed.

\section{The specialist's choice}

The specialist may wish to persuade the consumer to take one of two actions: to reappoint the administrator or terminate her tenure. This implies there are three plausible types of strategy that the specialist might use: to seek the administrator's reappointment by always indicating that quality is high, indicating quality is high or low when it is, or, to seek the administrator's termination by always indicating quality is low ${ }^{6}$

Let $Y_{0} \subseteq Y$ be the set of tenure decisions that the consumer could be persuaded to make. Let $Z^{S}\left(y, Y_{0}\right)$ be the set of $q$, given $w$, for which the specialist prefers $y$ over anything else in $Y_{0}$. The consumer can deduce that $q \in Z^{S}\left(y, Y_{0}\right)$ from the specialist's message. If $E\left[q \mid q \in Z^{S}\left(y, Y_{0}\right)\right] \in Z^{C}(y, Y)$ then the specialist persuades the consumer to choose $y$.

The specialist prefers the administrator's reappointment if his expected utility is greater under the incumbent than a replacement. Table 5.1 shows the specialist's preferred tenure decision, or the content of $Z^{S}\left(y, Y_{0}\right)$, for various values of $y$ and $Y_{0}$.

\begin{tabular}{|l|c|c|}
\hline When $Y_{0}$ is... & $\ldots$ then $S^{\prime}$ 's preferred $y \in Y_{0}$ is... \\
& $T$ when... & $R$ when... \\
\hline$\{T, R\}$ & $q<q^{\prime}+w^{\prime}-w$ & $q^{\prime}+w^{\prime}-w \leq q$ \\
$\{T\}$ & always & \\
$\{R\}$ & & always \\
\hline
\end{tabular}

Table 5.1: The specialist's choice

\footnotetext{
${ }^{5}$ In subgames where the consumer is uninformed, she will reappoint rather than terminate in equilibrium if it gives her a higher expected payoff: $\pi\left(q_{H}+q^{\prime}\right)+(1-\pi)\left(q_{L}+q^{\prime}\right) \leq \pi \cdot 2\left(q_{H}\right)+(1-\pi) \cdot 2\left(q_{L}\right)$, that is, $\frac{q^{\prime}-q_{L}}{q_{H}-q_{L}}=\pi^{*} \leq \pi$. This is equivalent to $q^{\prime} \leq E[q]$.

${ }^{6} \mathrm{~A}$ fourth, but implausible, pure strategy is that the specialist seeks termination of the administrator's tenure by indicating quality is low when it is high.
} 
The specialist will not necessarily prefer to reveal quality, as he cares about both quality and his wage 7 For example, if the administrator offers the specialist a sufficiently low wage then the specialist may expect better conditions under a replacement. The specialist might indicate strategically that quality is low, as an implicit recommendation to replace the incumbent administrator. The equilibrium effect of his message depends on its credibility and how the consumer responds to it.

\section{Wages and the specialist's incentives to reveal quality accurately}

We rule out implausible equilibria, such as babbling equilibria, and in section 5.3 .3 we establish when the administrator will wish to pay for informative messages. We foreshadow, here, what wages give the specialist an incentive to reveal quality accurately.

Wages in the following band imply that the specialist has an incentive to reveal quality:

$$
w^{\prime}+q^{\prime}-q_{H} \leq w<w^{\prime}+q^{\prime}-q_{L}
$$

We refer to these as 'signalling wages', and denote this set of wages $W_{\text {on }}=[\underline{w}, \bar{w})$. The consumer can deduce, for example, that the specialist would only want to recommend the administrator's reappointment if quality was high when $w \in W_{\text {on }}$. In this example, the consumer would update her beliefs according to Bayes' rule, so her belief that quality is high equals one when $m \in M_{H}$ and $w \in W_{\text {on }}$.

Wages below $\underline{w}$ or above $\bar{w}$ mean the specialist lacks the incentive to reveal quality accurately. We denote such a set of wages as $W_{\text {off }}$. For example, if the administrator sets a wage below $\underline{w}$, then the specialist expects a higher payoff under a new regime. As the specialist lacks the incentive to reveal quality, the consumer cannot deduce anything meaningful about $q$ from the specialist's message. Thus, when $w \in W_{\text {off }}$, the consumer would act on her prior beliefs, which equals the probability that quality is high, $\pi$.

\footnotetext{
${ }^{7}$ We assume for simplicity that if the specialist is indifferent over the administrator's tenure that he sends the administrator's preferred message. This assumption simplifies the analysis without substantially affecting our results. Appendix A.4.1 discusses an alternative assumption suggested by Farrell (1993).
} 
Figure 5.2 shows the wage bands and critical wage thresholds.

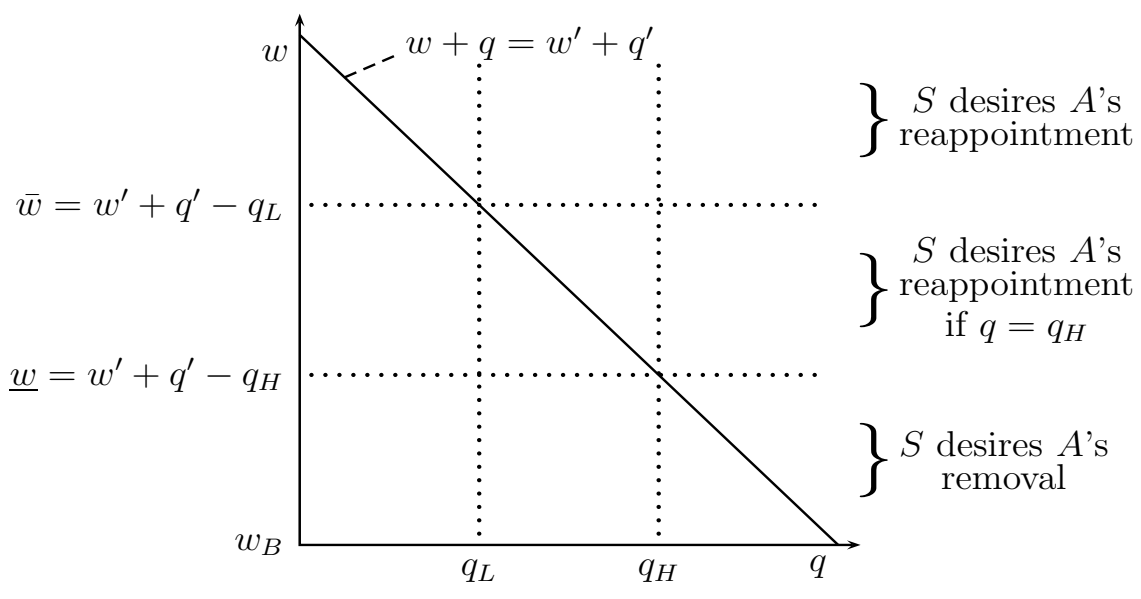

Figure 5.2: Critical wage bands

The negatively sloped line may be interpreted as an indifference curve for the specialist, where $w^{\prime}+q^{\prime}$ represents the specialist's reservation utility, given he has accepted the administrator's offer at step $\left.2\right|^{8}$ Points above the indifference curve give the specialist higher expected utility from the administrator's reappointment than her replacement. The three wage bands correspond to the three plausible types of strategy that the specialist may wish to use: indicating quality is always high, sometimes high or always low.

\section{Potential Perfect Bayesian Equilibria}

Below, we characterise potential PBE outcomes of the game. In section 5.3.3 we check that the sub-games of these PBE are plausible, and establish when the administrator wishes to pay a wage premium to ensure the specialist's messages are informative.

Table 5.2 on page 122 suggests putative PBE for various voting sets, $Y_{0}$, and shows the conditions for a given $Y_{0}$ to be consistent with a PBE. The first row corresponds to a separating equilibrium with informative messages, and the second two rows correspond to pooling equilibria with uninformative messages.

${ }^{8}$ The line in the figure is an example drawn for all conceivable values of $q$. Our model assumes quality is discrete and will take one of two values, $q_{L}$ or $q_{H}$. 
As $q_{L}<q^{\prime}<q_{H}$, we can express the two conditions in Table 5.2 for $Y_{0}=\{T, R\}$ as $E\left[q \mid q<q^{\prime}+w^{\prime}-w\right]=q_{L}$ and $E\left[q \mid q^{\prime}+w^{\prime}-w \leq q\right]=q_{H}$. These conditions are equivalent to $w \in[\underline{w}, \bar{w})=W_{o n} !^{9}$ In the other two outcomes, the consumer acts on her prior beliefs about the probability of high quality, $E[q]$. That is, when $\pi<\pi^{*}$ then $E[q]<q^{\prime}$ and when $\pi^{*} \leq \pi$ then $q^{\prime} \leq E[q]$.

\begin{tabular}{|c|c|c|}
\hline $\begin{array}{c}\text { When } Y_{0} \\
\text { is ... }\end{array}$ & $\begin{array}{c}\text {... then } S \text { can persuade } C \\
\text { to take the decision } \\
y \in Y_{0} \ldots\end{array}$ & $\begin{array}{c}\ldots \text { if } C \text { believes } S \text { 's best choice in } Y_{0} \text { is } \\
\text { consistent with her preferred choice } \\
E\left[q \mid q \in Z^{S}\left(y, Y_{0}\right)\right] \in Z^{C}(y, Y) .\end{array}$ \\
\hline \multirow[t]{2}{*}{$T, R$} & $T$ & $E\left[q \mid q<q^{\prime}+w^{\prime}-w\right]<q^{\prime}$ \\
\hline & $R$ & $q^{\prime} \leq E\left[q \mid q^{\prime}+w^{\prime}-w \leq q\right]$ \\
\hline$\{T\}$ & $T$ & $E[q]<q^{\prime}$ \\
\hline$\{R\}$ & $R$ & $q^{\prime} \leq E[q]$ \\
\hline
\end{tabular}

Table 5.2: Conditions for $Y_{0}$ to be consistent with a PBE outcome

Table 5.2 indicates that there may be an equilibrium with either informative or uninformative messages. This suggests that the specialist would not always be able to take advantage of his role as an information provider. While the separating equilibrium allows the specialist to influence the public, the pooling equilibrium outcome contrasts with the third criticism of the CHE reforms.

\subsubsection{Plausible equilibria with neologism-proof subgames}

The conditions in Table 5.2 do not guarantee that the potential equilibrium is plausible. For example, babbling equilibria are not ruled out. Below we apply an equilibrium refinement, neologism-proofness, to rule out such implausible equilibria. ${ }^{10}$ First, we outline when the specialist might want to send an unexpected message, that is a neologism, and

\footnotetext{
${ }^{9}$ For all $w \in[\underline{w}, \bar{w}), q-w<q^{\prime}+w^{\prime}$ if $q=q_{L}$ and $q^{\prime}+w^{\prime} \leq q-w$ if $q=q_{H}$. The consumer may infer that $q=q_{i}$ when the specialist sends the message $m \in M_{i}$, which conditions her expectations, $E\left[q \mid m \in M_{i}, w \in[\underline{w}, \bar{w})\right]=q_{i}$.

${ }^{10} \mathrm{~A}$ neologism is an unexpected, but credible (self-signalling) message (Farrell 1993). A subgame is neologism-proof if the specialist cannot devise a neologism that would cause the consumer to change her putative equilibrium strategy.
} 
when this would persuade the consumer to deviate from the putative equilibrium. Then we consider the game as a whole and outline when the administrator would be willing to pay to ensure the specialist's messages are credible.

Consider a putative equilibrium in which the consumer's actions do not depend on the specialist's message, that is, an equilibrium with either $Y_{0}=\{R\}$ or $Y_{0}=\{T\}$. Let $y^{\prime}$ represent an unexpected message, that is, a recommendation that the consumer would not comply with in the putative equilibrium outcome ${ }^{11}$ Let $Z^{S}\left(y^{\prime}, Y_{0}\right)$ be the set of $q$ for which the specialist prefers $y^{\prime} \notin Y_{0}$ to $y \in Y_{0}$.

The specialist should be able to convince the consumer to choose $y^{\prime}$ if the specialist would only want the consumer to believe it when it was true. Such a neologism should cause the consumer to update her belief about the actual value of $q, E[q \mid m]=E\left[q \mid q \in Z^{S}\left(y^{\prime}, Y_{0}\right)\right]$, and may disrupt the putative PBE.

The following condition summarises when a neologism recommending $y^{\prime} \notin Y_{0}$ can upset a PBE involving $Y_{0}$ :

$$
E\left[q \mid q \in Z^{S}\left(y^{\prime}, Y_{0}\right)\right] \in Z^{C}\left(y^{\prime}, Y\right)
$$

Table 5.3 outlines when the specialist can disrupt a putative PBE. This table is the result of applying condition (5.7) for particular values of $y$ to equations (5.3) and (5.4). That is, it shows the conditions for sets of $q$ to be self-signalling.

\begin{tabular}{|l|c|c|c|}
\hline $\begin{array}{c}\text { When } Y_{0} \\
\text { is. . }\end{array}$ & $\begin{array}{c}\ldots S \text { prefers } \\
y^{\prime} \notin Y_{0} \ldots\end{array}$ & $\begin{array}{c}\ldots \text { when there is a } \\
q \in Z^{S}\left(y^{\prime}, Y_{0}\right) \ldots\end{array}$ & $\begin{array}{c}\ldots \text { and } S \text { can convince } C \text { to choose } y^{\prime} \\
\text { if } E\left[q \mid q \in Z^{S}\left(y^{\prime}, Y_{0}\right)\right] \in Z^{C}\left(y^{\prime}, Y\right)\end{array}$ \\
\hline$\{T\}$ & $R$ & $w^{\prime}+q^{\prime}-w \leq q$ & $q^{\prime}<E\left[q \mid w^{\prime}+q^{\prime}-w \leq q\right]$ \\
\hline$\{R\}$ & $T$ & $q<w^{\prime}+q^{\prime}-w$ & $E\left[q \mid q<w^{\prime}+q^{\prime}-w\right]<q^{\prime}$ \\
\hline
\end{tabular}

Table 5.3: Conditions for deviating from a potential PBE outcome with $\left|Y_{0}\right|=1$

PBE outcomes with uninformative messages, that is with $Y_{0}=\{T\}$ or $Y_{0}=\{R\}$, would be vulnerable to neologisms when $E\left[q \mid w^{\prime}+q^{\prime}-w \leq q\right]=q_{H}$ or $E\left[q \mid q<w^{\prime}+q^{\prime}-w\right]=q_{L}$.

\footnotetext{
${ }^{11}$ Note that when $\left|Y_{0}\right|=2$ is consistent with a PBE outcome, no credible neologisms are possible.
} 
That is, an NPE of a cheap-talk subgame will have informative messages when $w \in W_{\text {on }}$, as as either $\left\{q_{L}\right\}$ or $\left\{q_{H}\right\}$ would be a self-signalling set. However, when $w<\underline{w}$ then a neologism recommending $y=R$ would not be credible as the specialist always prefers termination; $Z^{S}\left(R, Y_{0}=\{T\}\right)=\varnothing$. In this case, the specialist would never want to recommend the administrator's reappointment over her termination. Equivalently, when $\bar{w}<w$ then a neologism recommending $y=T$ would not be credible as the specialist always prefers reappointment; $Z^{S}\left(T, Y_{0}=\{R\}\right)=\varnothing$. Thus, the specialist would be unable to disrupt a putative equilibrium when $w \notin W_{\text {on }}$.

In Table 5.4, we characterise a set of conditions for a potential PBE with neologism-proof message/voting subgames. We use Table 5.3 to restrict the conditions in Table 5.2 so that the subgames in a putative equilibrium outcome are robust to potential neologisms, that is, the equilibrium outcome is plausible.

\begin{tabular}{|l|c|}
\hline A PBE with $Y_{0}=\ldots$ & $\ldots$ has subgames that are robust to neologisms if... \\
\hline$\{T, R\}$ & $w \in W_{\text {on }}$ \\
\hline$\{T\}$ & $E[q]<q^{\prime}$ and $w \notin W_{\text {on }}$ \\
\hline$\{R\}$ & $q^{\prime} \leq E[q]$ and $w \notin W_{\text {on }}$ \\
\hline
\end{tabular}

Table 5.4: Conditions for $Y_{0}$ to be consistent with a potential NPE outcome

A condition for $Y_{0}=\{T, R\}$ to be consistent with an NPE outcome is that $E[q \mid q<$ $\left.w^{\prime}+q^{\prime}-w\right]<q^{\prime} \leq E\left[q \mid w^{\prime}+q^{\prime}-w \leq q\right]$. This is only possible when $\underline{w} \leq w<\bar{w}$ as $q_{L}<q^{\prime}<q_{H}$. Thus, we summarise the conditions from the first row of Table 5.2 as $w \in W_{\text {on }}$ in Table 5.4 .

Figure 5.3 on page 125 is based on the conditions in Table 5.4 , and corresponds to the subgames of the three potential equilibrium outcomes identified above in Table 5.2 .

This figure represents the specialist's and consumer's actions. For example, when $w \in$ $W_{\text {off }}$ and $E[q]<q^{\prime}$, the consumer would always terminate the incumbent administrator's tenure. 


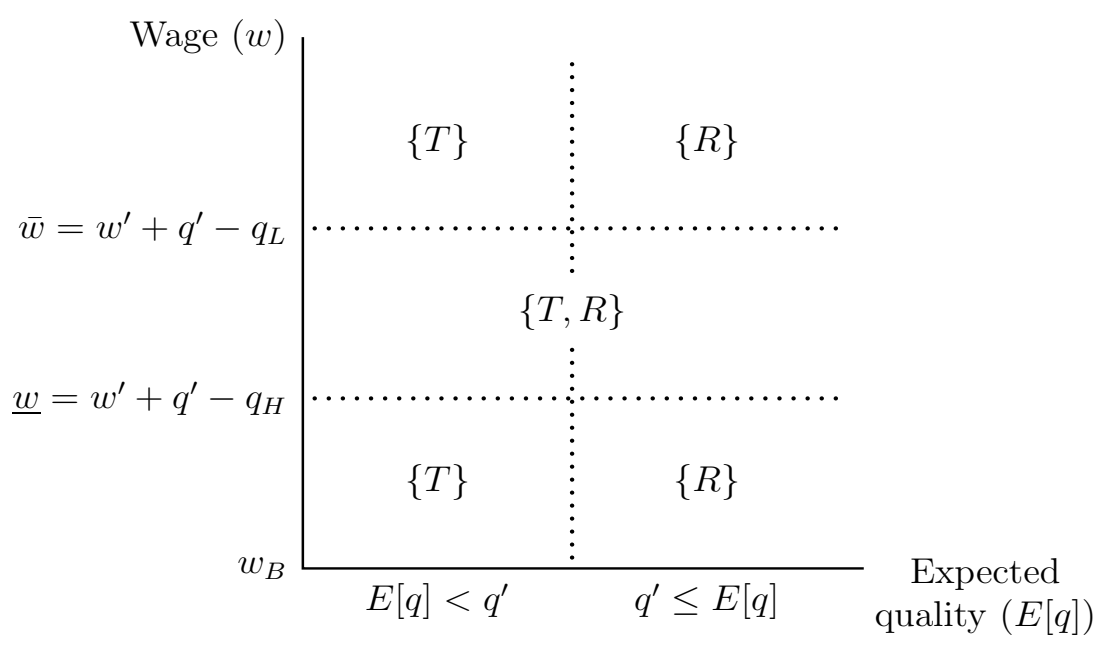

Figure 5.3: Outcomes in neologism-proof subgames

\section{The administrator's choice of the specialist's wage}

The conditions in Table 5.4 refer to the specialist's and the consumer's choices. These conditions are consistent with the specialist and consumer playing best responses in a message and voting subgame, given the administrator's choice of $w$. To identify an equilibrium for the game as a whole, we must also consider the administrator's choice of $w$ at step 1 .

\section{Informative messages in a separating NPE}

An informative message means that the consumer accurately learns the quality level. Such a message involves the administrator setting a wage that gives the specialist an incentive to reveal quality truthfully. However, it is not always in the interests of the administrator to provide such incentives.

Two conditions are required for the administrator to prefer the specialist's message to be informative. First, the administrator must face dismissal when the specialist's messages are uninformative. In this situation, the administrator may wish to offer a wage that will encourage the specialist to tell the truth. Second, there must be a wage that is high enough to ensure the specialist wishes to reveal the quality level truthfully yet low enough that the administrator is willing to offer it. 
The first condition is that the consumer's default choice would be to dismiss the incumbent administrator, that is, $\pi<\pi^{*}$. In this case, the administrator requires the specialist to provide a credible message in order for her to have a chance of reappointment. The administrator may achieve this by paying the specialist $w \in W_{\text {on }}{ }^{12}$

The second condition for an informative message to be part of an equilibrium strategy is that the administrator must be willing to pay for it. Although talk is cheap and the signal does not impose a direct cost on the specialist, a credible signal is costly to the administrator. The administrator has to pay the specialist a wage that is higher than the minimum possible wage, $w_{B}<w, \forall w \in W_{\text {on }}$. We assume that the administrator is willing to incur the signalling cost if it is necessary for reappointment:

$$
0 \leq G-\underline{w}
$$

If the consumer is pessimistic, then the administrator wants to reveal quality when $q=q_{H}$. The lowest wage the administrator can pay that is consistent with a credible message is $w=\underline{w}$. Therefore, to have a chance of staying in office, the administrator must pay the specialist a margin (in each period), $\underline{w}-w_{B}$, to secure a credible message ${ }^{13}$

Thus, in a separating NPE with informative messages, where $\pi<\pi^{*}$, the players' beliefs are driven by the structure of the game and they use the following strategies:

$$
\begin{aligned}
& A: w=\underline{w} . \\
& S: m \in\left\{\begin{array}{l}
M_{L} \text { if } w+q<w^{\prime}+q^{\prime} \\
M_{H} \text { otherwise. }
\end{array}\right.
\end{aligned}
$$

\footnotetext{
${ }^{12}$ Given the specialist's and the consumer's equilibrium strategies, the administrator is willing to turn the signal on if the expected benefit of reappointment is greater than the incremental cost of a credible message. This is true when $\pi<\pi^{*}$ if $w$ satisfies the following condition: $G-w_{B}<\pi(G-\underline{w})+(1-$ $\pi)(G-\underline{w}) \Rightarrow(1+\pi) \underline{w}-w_{B}<\pi G \Rightarrow \underline{w}<\frac{\pi}{1+\pi}\left(G+w_{B}\right)$.

${ }^{13}$ We concentrate on the case where $w_{B}<\underline{w}$, that is, where a signalling wage exceeds the minimum wage. We provide a rationale for this assumption in Appendix A.4.2. An alternative case is that $\underline{w}<w_{B}$. In this case, $\underline{w}$ is not feasible, and the administrator would have to pay the higher wage of $\bar{w} \geq w$ if she wants to turn the signal off. Although this alternative case would make informative messages cheaper than eroding the specialist's credibility with $\bar{w} \geq w$, the administrator would still face the risk of dismissal with the signal on. This provides some motivation for the administrator to wish to turn the signal off, but in this case the specialist would benefit from the erosion of his credibility.
} 


$$
C: y=\left\{\begin{array}{l}
T \text { if } w \notin[\underline{w}, \bar{w}), \text { or, if } m \in M_{L} \text { and } w \in[\underline{w}, \bar{w}) \\
R \text { otherwise. }
\end{array}\right.
$$

In a separating equilibrium, the administrator expects to be dismissed when the signal is off. She prefers to turn the signal on to have a chance of reappointment and is willing to pay $\underline{w}$ to achieve this. This wage is compatible with the specialist accurately reporting quality, which lets the consumer decide according to the quality level signalled.

\section{Uninformative messages in a pooling NPE}

The administrator may prefer uninformative messages when the consumer is sufficiently optimistic. She may choose a wage that undermines the specialist's incentive to tell the truth, which erodes his credibility with the consumer. This takes advantage of a consumer's inability to verify the accuracy of the specialist's message and knowledge of the incentives on the specialist to reveal quality accurately.

Two conditions are required for the administrator to prefer uninformative messages. First, the administrator must expect reappointment even if the specialist's message is uninformative. This is the case where the consumer's default choice is to reappoint the incumbent administrator, $\pi^{*} \leq \pi$.

The second condition for the administrator to prefer uninformative messages is that the specialist's wage is low enough so that the administrator prefers to be reappointed. Wages in either band, $w<\underline{w}$ or $\bar{w}<w$, turn the signal off. The wage bill for an uninformative message is lower if the administrator chooses a wage in the lower band ${ }^{14}$ The assumption that $w_{B}<G$ ensures that the second condition is satisfied and that the administrator would prefer to be reappointed.

A necessary condition for a pooling NPE with uninformative messages is that $\pi^{*} \leq \pi$. An example of this type of equilibrium is that the players use the following strategies, with

\footnotetext{
${ }^{14}$ The administrator's choice of wage from either the low or high band does not reveal any information about quality to the consumer as the administrator does not observe quality before setting the wage. That is, ceteris paribus, any of the wage offers that result in uninformative messages, $w \in W_{\text {off }}$, should have the same effect via Bayes' rule on the consumer's interpretation of the specialist's messages.
} 
beliefs driven by the probability distribution of quality:

$A: w=w_{B}$.

$S: m \in\left\{\begin{array}{l}M_{L} \text { if } w+q<w^{\prime}+q^{\prime} \\ M_{H} \text { otherwise. }\end{array}\right.$

$C: y=\left\{\begin{array}{l}T \text { if } m \in M_{L} \text { and } w \in[\underline{w}, \bar{w}) \\ R \text { otherwise. }\end{array}\right.$

In a pooling equilibrium, an optimistic consumer, $\pi^{*} \leq \pi$, would reappoint the incumbent when she is uninformed. The administrator maximises her payoff by paying $w_{B}$. By eroding the specialist's credibility, the administrator avoids paying a higher (signalling) wage and the risk of the specialist revealing that quality is low and her consequent dismissal. The specialist, however, is unable to benefit from his role as an information provider. The consumer also suffers from the lack of information as she fails to dismiss the administrator when quality is low.

\section{Plausible NPE outcomes}

Below we check to see whether the three potential PBE outcomes characterised above are consistent with the administrator making a utility maximising choice of $w$. That is, we check that the administrator's choice is consistent with her playing a best response as part of an NPE outcome for the entire game.

The first line of Table 5.4 presents conditions that would need to be satisfied in an informative NPE. In such an NPE, the administrator prefers the specialist to send credible messages, as she would otherwise be dismissed. When $\pi<\pi^{*}$, the administrator sets $w=\underline{w}$. In this outcome, the specialist can always persuade the consumer to believe that quality is either high or low, and there are no neologisms that could upset the putative equilibrium as $\left|Y_{0}\right|=2$. Therefore, $Y_{0}=\{T, R\}$ is consistent with an NPE outcome when $\pi<\pi^{*}$. 
In the other two outcomes detailed in Table 5.4, the administrator sets the specialist's wage so as to erode his credibility, that is, $w \in W_{\text {off }}$. A necessary condition for the administrator to prefer an uninformative message is $\pi^{*} \leq \pi$. In this type of outcome, although the specialist might wish to persuade the consumer to deviate from her putative choice, he is unable to devise a credible neologism. That is, because of the specialist's wage, the consumer does not believe their interests are sufficiently aligned for the specialist's messages to be credible.

The second line, $Y_{0}=\{T\}$, corresponds to an outcome where the consumer always expects the specialist to recommend removing the administrator. In this outcome, the consumer would act on her prior beliefs and dismiss the administrator, as $E[q]<q^{\prime}$. However, $w \in w_{\text {off }}$ is not a utility-maximising choice for the administrator, as she would prefer to pay to turn the signal on than to be terminated for sure, as $0 \leq \underline{w}<G$. Therefore, $Y_{0}=\{T\}$ is not consistent with an NPE outcome.

The third line, $Y_{0}=\{R\}$, corresponds to an outcome where the specialist cannot persuade the consumer to terminate the administrator's tenure. This outcome is only neologismproof when $q^{\prime}<E[q]$. As the administrator would always be reappointed in this outcome, her utility maximising choice is to pay the minimum wage, $w=w_{B}$. Therefore, $Y_{0}=\{R\}$ is consistent with an (uninformative) NPE when $\pi^{*} \leq \pi$.

The equilibrium outcome is unique as the conditions for an equilibrium consistent with $Y_{0}=\{T, R\}$ or $Y_{0}=\{R\}$ are mutually exclusive.

\subsubsection{Results}

We embed cheap talk subgames in multi-layered organisational hierarchy model, which is an innovation on existing literature. We use this model to examine when a specialist has an opportunity to take advantage of soft information on quality to influence the public. We conclude that there is a unique NPE outcome. The type of equilibrium depends on $\pi$. It can be either a separating equilibrium (with informative message), when $\pi<\pi^{*}$, or a pooling equilibrium (with uninformative message), when $\pi^{*} \leq \pi$. 
An important insight of this model is that the administrator can influence the specialist's credibility. The administrator can set the specialist's remuneration so as to ensure or erode his credibility. The separating equilibrium result indicates that, in some circumstances, administrators may have preferred that specialists provide consumers with information. In this type of outcome, the specialist benefits from his role as an information provider in the sense that he earns a wage above the minimum possible. But it is in the administrator's interests to permit the specialist to carry out this role.

Our results suggest that medical specialists in the CHE reforms might not have had the opportunity to communicate effectively with, and manipulate, the public. In the pooling equilibrium outcome with uninformative messages, the specialist does poorly by being held to his minimum wage, and consumers are left making poorly informed decisions. Thus, although reformers may have aimed to improve the system's performance by introducing market disciplines administered by cost-conscious CEOs, these managers potentially had perverse incentives to manipulate specialists to the detriment of both specialists and the public.

In the next section, we consider how managerial effort to redesign the hospital's operations, which was an important feature of the reforms, might also have created perverse incentives for managers.

\subsection{Managerial effort and public expectations}

The model in the previous section identified a role for the specialist as an information provider. In this section, we explore how the administrator might take advantage of her own role to reduce her dependence on the specialist. We incorporate an important feature of the CHE reforms: CEOs were expected to reform management and information systems in hospitals. The result, that the administrator can take advantage of her role, contrasts with the third criticism of the CHE reforms.

We modify the model in section 5.3 so that the administrator can reduce her dependence 
on the specialist as a conduit of information. We let the administrator take some preliminary action that increases the probability of high quality. The administrator and the specialist then negotiate a wage in light of the administrator's commitment.

These modifications allow the administrator to make a credible commitment that gives her more influence. In particular, the administrator has an alternative way to influence the consumer's beliefs in addition to manipulating the specialist's credibility. This reduces her need for the specialist's message to be informative ${ }^{15}$ As such, the administrator's effort allows a pooling equilibrium under a wider range of circumstances than in the previous section, where the administrator implicitly provided no effort. That is, now the administrator may use her effort to directly manipulate the consumer, which may permit the administrator to secure reappointment without turning the signal on.

\subsubsection{Play of the game}

We add an initial step in which the administrator commits to a level of effort, $e \in\{0,1\}$. The administrator's effort increases the likelihood that quality is high, $\pi(0)<\pi(1)$; $e$ is public knowledge ${ }^{16}$ The specialist observes $e$, and decides in step 3 whether to accept the administrator's offer. If the specialist accepts the wage, the game continues from Nature's move in much the same way as in section 5.3 .

The steps of the game are as follows:

1. A chooses $e \in\{0,1\}$, which influences $\pi(e)$.

\footnotetext{
${ }^{15}$ Another criticism of the CHE reforms, which is not directly addressed here, provides a reinforcing motivation as to why an administrator might prefer uninformative messages. More than half of the CEOs appointed came from outside the health sector (Troughton 1993). Some commentators have suggested that a lack of health sector experience of these new CEOs could have contributed to the poor performance of the CHEs (Easton 1997, Boston et al. 1999). If experience working in the health was important to managing public hospitals, then new CEOs may have wanted to redirect attention from their lack of experience. Such actions would be consistent with an equilibrium result in this model where the administrator makes some strategic commitment, turns the signal off and is reappointed.

${ }^{16}$ In the case of the health reforms, a hospital manager's effort might manifest in a number of ways, such as developing (and publicising) a business plan for a hospital's operation. This could improve the public's perception of the hospital manager's control of the organisation, or the manager's competence at addressing rationing or perceived resource allocation problems within hospitals.
} 
2. $A$ chooses a wage to offer $S, w \in\left[0, w_{G}\right]$.

3. $S$ observes $e$ and $w$, and decides whether to accept the offer, $O$. If he rejects it, $Q$, then the player's receive their reservation utilities, $\bar{u}_{i}, i \in\{A, S, C\}$. Otherwise ...

4. $N$ chooses the organisation's quality level, $q \in\left\{q_{L}, q_{H}\right\}$, where $P\left(q=q_{H}\right)=\pi(e)$.

5. $S$ observes $q$ (as well as $e$ and $w$ ), and chooses a message, $m \in M$.

6. $C$ observes $e, w$ and $m$. She does not observe $q$, before making her decision. She decides whether to reappoint $A(\mathrm{R})$ or terminate her contract (T).

Figure 5.4 illustrates the steps of the game, where the players' payoffs are specified in array 5.9 on page 133 .

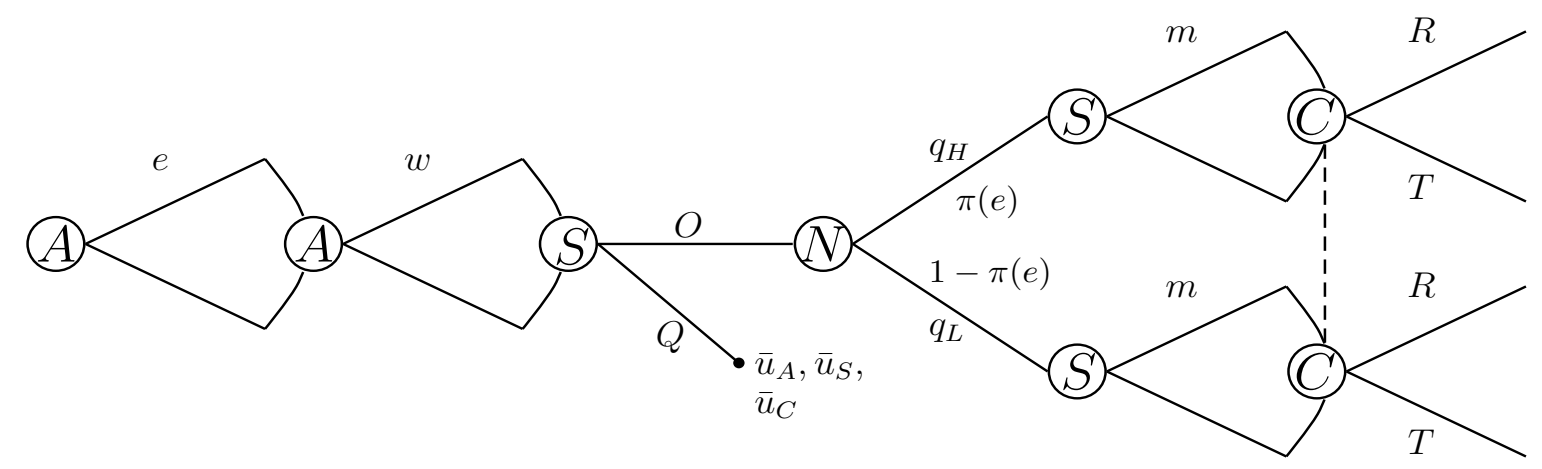

Figure 5.4: Game tree for three-actor model with commitment step

The administrator's choice of whether to have informative messages depends on the effect of her effort on the consumer's optimism. 17 In sub-games where the specialist's messages are uninformative, the consumer can condition her belief about $\pi$ on $e$, consistent with Bayes' rule. By increasing the probability of high quality via her effort, the administrator increases the likelihood that the consumer will reappoint her without having to pay for an informative message.

\footnotetext{
${ }^{17}$ Although the consumer's prior beliefs are driven by the structure of the game, for convenience we describe the consumer as optimistic if $\pi^{*} \leq \pi$ and pessimistic if $\pi<\pi^{*}$.
} 
Except for the cost of the administrator's effort, the payoffs are the same as for the game in section 5.3 ;

$$
\begin{aligned}
& U_{A}=\left\{\begin{array}{l}
2(G-w)-e \text { if reappointed } \\
G-w-e \text { otherwise }
\end{array}\right. \\
& U_{S}=\left\{\begin{array}{l}
2(w+q) \text { if } A \text { reappointed } \\
w+q+w^{\prime}+q^{\prime} \text { otherwise }
\end{array}\right. \\
& U_{C}=\left\{\begin{array}{l}
2 q \text { if } C \text { reappoints } A \\
q+q^{\prime} \text { otherwise }
\end{array}\right.
\end{aligned}
$$

As in the previous version of the model, the specialist will accept the administrator's offer when the wage is above a reservation level, $w_{B}$. We assume that the administrator is willing to incur some cost if it is necessary for reappointment:

$$
0<G-w_{B}-e
$$

This assumption means that the administrator's net payoff may be positive, including when she provides effort. The assumption is similar to, though not necessarily stronger than, assumption (5.8) in section 5.3.

\subsubsection{Solving the game}

The administrator's effort and signalling choices depend on the impact of her effort on the consumer's beliefs about $\pi$. We use backwards induction to characterise these choices in equilibrium.

\section{The reappointment decision}

At step 5 in Figure 5.4. Nature has determined the quality level. If the quality signal is on, that is the specialist's message is credible, then the consumer learns what the quality level is and makes her choice accordingly. 
If the signal is off, the consumer's reappointment choice depends on her beliefs, conditioned on $e$, relative to her critical threshold $\pi^{*}$. This threshold was defined in section 5.3. The consumer would dismiss the administrator if she is pessimistic, $\pi(e)<\pi^{*}$, or reappoint the administrator if she is optimistic, $\pi^{*} \leq \pi(e)$.

Below we examine three cases where $\pi^{*}$ could fall below, between or above $\pi(0)$ and $\pi(1)$.

\section{The effort and signalling decisions}

At step 2 in Figure 5.4, the administrator will only pay for an informative message if it is worth the expense ${ }^{18}$ Recall that a message is informative if the specialist has an incentive to reveal quality accurately, and the minimum wage consistent with informative messages is $\underline{w}$.

- Case 1: $\pi(0)<\pi(1)<\pi^{*}$

When $\pi(0)<\pi(1)<\pi^{*}$ the consumer is pessimistic, and the administrator cannot sway her reappointment decision with effort alone. Nonetheless, effort would increase the administrator's chance of reappointment by increasing the probability of high quality.

The administrator has an incentive to pay for an informative message in this case, and may also want to put in high effort under some conditions, which we explore below. As in the informative equilibrium in section 5.3, the administrator pays the specialist to provide informative messages in order to have a chance of reappointment. The specialist can persuade the consumer to believe that quality is either high or low, so there are no neologisms that could upset the putative equilibrium.

The equilibrium has two necessary conditions, analogous to those in section 5.3 on page

\footnotetext{
${ }^{18}$ For the administrator to prefer to pay for a credible message, it must be required for re-election, i.e. $\pi(e)<\pi^{*}$, and not be so costly as to outweigh the benefit of reappointment. The wage decision is made independently of the effort choice, as at step 2 . effort is a sunk cost. If the administrator turns the signal on, her expected payoff is $\pi(e)(2(G-\underline{w})-e)+(1-\pi(e))(G-\underline{w}-e)$, which simplifies to $(1+\pi(e))(G-\underline{w})-e$. If the administrator turns the signal off, then her expected payoff is $G-w_{B}-e$ if $\pi(e)<\pi^{*}$. Given the specialist's and the consumer's equilibrium strategies, the administrator is willing to turn the signal on when $\pi(e)<\pi^{*}$ if $w$ satisfies the following condition: $G-w_{B}-e<$ $(1+\pi(e))(G-\underline{w})-e \Rightarrow(1+\pi(e)) \underline{w}-w_{B}<\pi(e) G \Rightarrow \underline{w}<\frac{\pi(e) G+w_{B}}{1+\pi(e)}$.
} 
126. The first of these conditions relates to the need for the administrator to pay for a signal to have a chance of reappointment, namely $\pi(e)<\pi^{*}$. The second condition is qualitatively the same, and requires a signalling wage to be cost-effective from the administrator's perspective. The condition is modified to reflect the impact of the administrator's effort and is $\underline{w}<\frac{\pi(e)}{1+\pi(e)}\left(G+w_{B}\right) 19$

Although the administrator's payoff does not directly depend on quality, effort increases the probability of $q_{H}$ and therefore her probability of reappointment. In the present case where $\pi(0)<\pi(1)<\pi^{*}$, the administrator's expected payoff is higher with high effort than with low effort under the following condition:

$$
\pi(0) 2(G-\underline{w})+(1-\pi(0))(G-\underline{w})<\pi(1) 2[G-\underline{w}]+(1-\pi(1))(G-\underline{w})-1 .
$$

This condition can be simplified as follows:

$$
1<(\pi(1)-\pi(0))(G-\underline{w})
$$

If this condition is satisfied, then the administrator will provide effort and turn the signal on in equilibrium. However, the administrator's decision about effort does not necessarily align with a socially optimal outcome. As effort increases the probability of high quality, the administrator's effort affects the specialist's and the consumer's payoffs. The administrator's effort choice would be socially efficient if her private benefit were calibrated to society's benefit. In principle, the funder could align the administrator's incentives to maximise total surplus by negotiating a remuneration package so that $G=2 q_{H}-\left(q_{L}+q^{\prime}\right) .^{2021}$

\footnotetext{
${ }^{19}$ In equilibrium, the administrator is willing to turn the signal on if the expected benefit of reappointment is greater than the incremental cost of a credible message. This is true when $\pi(e)<\pi^{*}$ if $w$ satisfies the following condition: $G-w_{B}<\pi(e)(G-\underline{w})+(1-\pi(e))(G-\underline{w}) \Rightarrow \underline{w}<\frac{\pi(e)}{1+\pi(e)}\left(G+w_{B}\right)$.

${ }^{20}$ If $G$ and $w$ are pure transfers, total surplus plausibly equals the quality level in each period minus effort. Then, effort maximises total surplus under the following condition:

$$
\pi(0)\left(2 q_{H}\right)+(1-\pi(0))\left(q^{\prime}+q_{L}\right)-0<\pi(1)\left(2 q_{H}\right)+(1-\pi(1))\left(q^{\prime}+q_{L}\right)-1 .
$$

This condition can be simplified as follows.

$$
1<(\pi(1)-\pi(0))\left[2 q_{H}-\left(q_{L}+q^{\prime}\right)\right]
$$

This condition indicates that effort is efficient if the increase in expected quality exceeds the marginal cost of effort.

${ }^{21}$ This simple characterisation of total surplus focuses on the alignment between an administrator's
} 
- Case 2: $\pi^{*} \leq \pi(\mathbf{0})<\pi(\mathbf{1})$

At the other extreme to case 1 , if the consumer is sufficiently optimistic she would reappoint the administrator in the absence of an informative message from the specialist. The administrator avoids the risk of being dismissed by paying a wage that makes the specialist's messages uninformative. The administrator can erode the specialist's credibility with the consumer and minimise the wage bill by paying $w_{B}$. Such a wage means the specialist lacks the incentive to report quality accurately when $q=q_{H}$, thereby eroding his credibility.

In the initial step, the administrator's utility is higher without providing costly effort. She is guaranteed reappointment, so receives no benefit from providing effort or paying for an informative message.

This case is equivalent to the uninformative NPE in section 5.3 , but with the inclusion of an effort step. The equilibrium outcome delivers $Y_{0}=\{R\}$. As the administrator offers the specialist a wage of $w_{B}$, the specialist would never want to indicate that quality is high, which would be an implicit recommendation to reappoint the administrator. Therefore, there are no credible neologisms that could disturb this equilibrium.

The consumer receives too little information, in the sense that she reappoints the administrator even when quality is low. In addition, the administrator's effort may be inefficiently low. That is, the administrator expects to be reappointed without providing effort so the private marginal benefit of effort is zero. But effort would be efficient where the social marginal benefit of effort exceeds its social marginal cost, $1<(\pi(1)-\pi(0))\left[2 q_{H}-\left(q_{L}+q^{\prime}\right)\right]$, as noted in footnote 5.4 .2 .

- Case 3: $\pi(\mathbf{0})<\pi^{*} \leq \pi(\mathbf{1})$

In this case, when the signal is off, the consumer will dismiss the administrator if effort was low, but reappoint if effort was high.

private incentives for effort and society's preferences. While this is a common simplification, it ignores issues such as any deadweight loss that results from raising funding with taxes. 
Thus in the initial step, the administrator has a substantive choice about providing effort versus paying for an informative message. Table 5.5 summarises the probability of reappointment for various combinations of effort and the wage.

\begin{tabular}{|c|c|c|}
\cline { 2 - 3 } \multicolumn{1}{c|}{} & $w \in W_{\text {on }}$ & $w \in W_{\text {off }}$ \\
\hline $\mathrm{e}=1$ & $\pi(1)$ & 1 \\
\hline $\mathrm{e}=0$ & $\pi(0)$ & 0 \\
\hline
\end{tabular}

Table 5.5: The probability of reappointment in case 3 given effort and the wage

Thus, the administrator's effective decision is between $e=1, w=w_{B}$ and $e=0, w=\underline{w}$. The administrator will provide effort, and turn the signal off, if the cost of effort is outweighed by the greater chance of reappointment. This is expressed mathematically in the following condition:

$$
\pi(0) 2(G-\underline{w})+(1-\pi(0))(G-\underline{w})<2\left(G-w_{B}\right)-1 .
$$

This simplifies as follows:

$$
1<(1-\pi(0)) G+(1+\pi(0)) \underline{w}-2 w_{B} .
$$

The administrator can secure reappointment without paying for informative messages, but only with high effort. If the cost of effort is sufficiently high, however, her expected payoff may be higher with informative messages and no effort.

This case is a variant of the two NPE outcomes in section 5.3 where either $Y_{0}=\{T, R\}$ or $Y_{0}=\{R\}$. The administrator may prefer a signal over effort, and would set $w=\underline{w}$. In this case, the specialist's messages are credible and there are no neologisms that could upset the putative equilibrium where $Y_{0}=\{T, R\}$. On the other hand, the administrator may prefer to provide effort and to reduce her dependence on the specialist. In this case, the administrator would erode the specialist's credibility by paying $w=w_{B}$. Such a wage rules out any credible neologisms, and corresponds to the neologism-proof outcome with $Y_{0}=\{R\}$ 
When $\pi(0)<\pi^{*} \leq \pi(1)$ either type of equilibrium outcome may occur, depending on the relative impact of effort and the cost of the signal. In a pooling equilibrium outcome, when condition 5.12 holds, the administrator modifies the consumer's prior beliefs with her effort, but she prevents informative communication by eroding the specialist's credibility. In the separating equilibrium outcome, effort will be low and the message will be informative. Both outcomes involve a trade-off so neither may be socially optimal: either effort or communication could be inefficiently low.

\subsubsection{Results}

A key result of our model is that sometimes the administrator wants to provide effort and to prevent meaningful communication. This result provides a more sophisticated insight than is suggested by the third criticism of the CHE reforms. Although we find that the specialist might have an opportunity to influence the public, the administrator's role and incentives within the stylised CHE might also have given her the opportunity to influence the public to the detriment of specialists and the public.

The administrator's effort makes her less dependent on the specialist than under the model in section 5.3. An administrator who is sufficiently confident about her ability to deliver reforms that will be well-regarded by the public has an incentive to combine effort with reduced remuneration or conditions for medical staff. That is, the administrator could benefit from leveraging her effort and reducing meaningful communication.

Our analysis provides an alternative view of some of the third criticism of the reforms. The key result above contrasts with the criticism that specialists manipulated their roles as service and information providers to their own benefit. Under the reformed CHEs, the specialists' role as providers of information to the public may have increased in importance. However, through setting wages or providing effort, administrators may have limited the ability of specialists to take advantage of their role as information providers. This may have also resulted in inefficiently low communication with consumers. 
The results in this section amplify the finding of Chapter 4 , where strong incentives for cost control have the potential to increase costs as a result of poor information flow. This section demonstrates that an administrator introduced to improve an organisation's operation may face perverse incentives to prevent the flow of beneficial information.

\subsection{Discussion}

This chapter developed two models to consider the third criticism of the reforms: that the reforms gave medical specialists the opportunity and incentives to manipulate managers to the detriment of hospital efficiency.

We use an innovative modelling approach by embedding cheap talk subgames in a multilayered organisational hierarchy and applying the concept of neologism-proofness to identify plausible equilibria. In our first model, we show that a superior agent in an organisation may prefer to encourage the communication of soft information. Our second model expands the first to allow for managerial effort, and shows how a superior agent might benefit from preventing communication. This second result reinforces our finding of Chapter 4 that a principal may face a tradeoff between sharp incentives for performance by an administrator and soft incentives that promote communication, here between the specialist and the consumer.

The first model examined when an administrator would pay for a costly signal that informs the consumer about quality. The administrator can manipulate the specialist's wage to influence his credibility, thereby affecting the information he can successfully transmit to the consumer about quality. In a pooling equilibrium, the administrator prefers to turn off the flow of information and to hold the specialist to his minimum possible wage. This suggests that medical specialists in the CHE reforms did not necessarily benefit from the increased prominence of their role as providers of information as well as medical services. However, the CHE reforms may have raised consumers' fears of cost-cutting and quality reductions. Such a situation could correspond with the separating equilibrium, where the 
administrator relies on the specialist to provide credible information to the public.

In the modified model, the administrator can avoid relying so heavily on the specialist by substituting effort for information. Under particular conditions, the administrator prefers to secure reappointment by putting in effort, thereby making the consumer more optimistic. In this case, the administrator can avoid the cost of turning the signal on, and erodes the specialist's credibility.

A central thread of the CHE reforms was to reduce the alignment between the interests of hospital managers and medical specialists, which some of the reformers felt were too closely aligned. Managers who conformed with the new corporate agenda, and who typically came from outside the health sector, were appointed to drive the reforms home within CHEs. This included putting managerial effort into designing and implementing new processes in a CHE, and relying less on medical specialists in managerial decision-making processes.

As we show in the second model, managerial effort could erode the specialist's role as an information provider, resulting in a pooling equilibrium with uninformative messages. Such an outcome is not always in the public's interest, as the uninformed public fails to dismiss the administrator when quality is low.

This chapter shows that the reforms did not necessarily give specialists the opportunity to manipulate the system. We show that specialists might have done poorly. Furthermore, the introduction of managers expert in innovation could have allowed them to disrupt the specialists' role as information providers, and to silence the whistle-blower. As shown in the second model, this may not be in the public's interest as it could lead to inefficiently low effort or an inefficiently low transfer of information.

The concluding chapter draws together the results from this chapter with the results of the earlier chapters. It notes how the findings in each chapter provide a mutually reinforcing explanation of how a set of reforms designed to improve a failing system could fail to achieve even their primary objective. 


\section{Chapter 6}

\section{Conclusion}

The health reforms have yet to yield the original expectations.

- Crown Company Monitoring Advisory Unit (1997, 21)

The CHE reforms aimed to control costs and improve operational efficiency. One action the reformers believed would achieve this goal was to increase tension between management and clinicians in public hospitals. The sharp incentives for performance created for CEOs were expected to flow down through the hospital hierarchy, improving performance at all levels.

Although the reforms may have succeeded in creating tension within the public hospital system, the reforms had unanticipated effects that generated substantial criticism of the system. Ultimately, these criticisms contributed to the replacement of the corporatised CHEs with non-profit hospitals. New Zealand's health reformers may have initially incorrectly estimated the risk profile of the population. In this case, the reformers may have believed that pursuing the reforms would reduce treatment cost and improve efficiency. Instead, our model suggests how sharper managerial incentives could have led to an outcome with increased costs. In the light of inflating costs and flagging service quality, the CHE reformers may have realised this error and retracted the corporate model, thereby relaxing the administrators' sharp cost control incentives. Waikato Hospital, for example, introduced specific initiatives aimed at fostering cooperation between managers and 
specialists, which contributed to the CHE regaining control of its finances and taming its deficit (Mold 1996).

This thesis formally examines three of the major criticisms of the CHEs.

1. CHE Boards failed to provide strong incentives and paid managers bonuses despite failing to achieve performance targets.

2. Although managers were given strong incentives for cost control, costs escalated.

3. The reforms gave medical specialists the opportunity and incentives to manipulate managers politically to the detriment of hospital efficiency.

We use a stylised characterisation of a CHE to analyse formally the interactions in a multi-layered organisational hierarchy that could lead to these criticisms.

We introduce the criticisms in Chapter 1, as well as providing the general context of the CHE reforms and a review of the relevant theoretical literature to which this thesis adds.

Chapter 2 complements the backdrop provided in Chapter 1. It econometrically tests whether the CHE reforms were independently associated with increases in treatment costs, or whether changes in treatment costs might have reflected coincident changes in other cost drivers, such as patient demographics or a background trend in medical technology. The empirical analysis finds that the CHE reforms increased treatment costs within New Zealand's public hospitals. This finding motivates the theoretical analyses.

Chapter 3 builds on Chapter 2 and considers how a funder might incentivise an administrator to pursue a tighter budget target in an uncertain environment where budgets could blow out despite the administrator's efforts. It examines the mechanisms a funder might use to encourage efficient, delegated decision making focussed on cost control.

While imposing an HBC on an agent can be desirable, this is not always the case. We generate the novel result that budget flexibility paired with bonuses can be efficient in the light of uncontrollable environmental conditions, rather than a sign of poor management. 
The flexibility in an FBC regime can enhance an administrator's ex ante incentives to provide the funder's desired level of effort, while also encouraging ex post efficient decisions in light of actual conditions. The incentives required in a simple organisational hierarchy, however, may not be sufficient in a complex organisational hierarchy like a CHE.

One aim of the reforms was that efficiency incentives given to CEOs should pass down the organisational hierarchy to subordinate levels. Chapter 4 considers the interaction between a manager, who focuses on cost control, and a specialist, who has better information about patients than the manager. We argue that the reforms created sharp performance incentives for managers by manipulating the CHEs' budgets and CEOs' bonuses.

Our model in Chapter 4 shows how sharp incentives may erode an agent's credibility and reduce the meaningful communication of soft information. Realigning an administrator's interests towards those of the funder, could also create a misalignment between the administrator and the specialist. We show that such a misalignment could leave the administrator less well informed so she makes poorer rationing decisions. We argue that sharpening an administrator's incentives for cost control can impair communication and can cause costs to rise. This result seems plausible where the administrator anticipates the funder will be flexible about the budget, so that the administrator would prefer to blow the budget rather than risk under-treatment.

Chapter 5 examines how incentives created by reforms to the top of the organisational hierarchy could affect the interaction between specialists and consumers at the coalface of the organisation. While CEOs may have lost access to some important information from specialists, the reforms may have unexpectedly increased the importance consumers placed on specialists as a credible source. Although specialists might have benefitted such a role, the reforms may have given an administrator the opportunity and incentives to hinder the information flow to consumers. By manipulating the working conditions of specialists, managers might erode the credibility of specialists' messages to consumers. This reduces meaningful communication, to the detriment of specialists and consumers.

In the context of the CHEs, the reformers aimed to change the behaviour throughout 
a hospital's hierarchy by sharpening performance incentives at the management layer. But the reformers may not have accurately anticipated the impacts of this tension in an environment where information is an important resource but its communication may be impaired by strong incentives. Our results provide insight into three criticisms of the CHE reforms. Tension and perverse incentives could have caused costs to rise, necessitating budget revisions and additional bonus payments, while permitting administrators to silence the whistle-blowers.

There are a number of useful extensions that could be made, particularly to the core cheap talk model in Chapter 4. Our cheap talk model abstracts from how people enter the system; that is, how patients choose to access the public hospital system. One extension is to endogenise a consumer's choice to enter the system. If people are aware of their individual risk, the entry choice may be biased resulting in adverse selection. Adverse selection may raise the administrator's prior belief about a patient's risk, resulting in more cautious rationing in the absence of credible information.

Chapter 3 assumed that CHE managers had a uniform impact on the organisation's operation. However, the CHE reforms introduced new managers, many of whom came from outside the health sector. These new managers may have had less understanding of, or empathy with, the medical establishment and patient outcomes. They may also have been less effective due to a lack of health sector-specific knowledge or because of professional divides between the cultures of management and medicine.

The CHE boards, however, retained some managers who were involved with public hospitals prior to the reforms. Further investigation could establish the relative importance of incentive effects and manager type to explain the relative performance of CHEs. The investigation could examine whether CEO performance was uniform across reappointed managers, with health sector expertise, and those new to the sector, with a comparative strength in administrative and managerial services. Such an investigation could inform an analysis of New Zealand's current District Health Board system, especially with moves to centralise some administrative and purchasing services in a central services agency. 
Chapter 5 considered how specialists might have had the opportunity and incentives to manipulate the public using a novel mechanism related to the communication of soft information to influence patient's beliefs about service quality. An extension to our model could draw on Vaithianathan's (1999b, 176-77) conclusion that medical specialists had incentives to manipulate the supply of public hospital services. The extension could endogenise the medical labour supply. The results of such work are likely to yield valuable insights into complex organisational forms within a multi-sector environment.

The insights from this thesis extend beyond the health sector. This thesis adds to the literature on incomplete contracting, particularly in terms of the communication of soft information in multi-layered organisational hierarchies. We show that a principal may face a tradeoff between sharp incentives for performance and soft incentives that promote communication in a complex organisational hierarchy.

The core results could be applied to sectors where the communication of soft information is important for a complex organisation to function efficiently. This is particularly relevant to service organisations, or research and development settings, where ideas are poorly defined but not necessarily difficult to communicate. Success in such settings requires systems that enhance trust and collaboration, rather than create unhealthy tension. 


\section{Bibliography}

Aghion, P. \& Tirole, J. (1997), 'Formal and real authority in organizations', Journal of Political Economy 105, 1-28.

Alam, M. \& Lawrence, S. (1994), 'A new era in costing and budgeting: implications of health sector reform in New Zealand', International Journal of Public Sector Management 7(6), 41-51.

Alchian, A. A. \& Demsetz, H. (1972), 'Production, information costs, and economic organization', The American Economic Review 62(5), 777-795.

Arrow, K. J. (1963), 'Uncertainty and the welfare economics of medical care', The American Economic Review 53(5), 941-973.

Arthur Anderson and Co (1987a), Public hospital performance assessment, Wellington.

Arthur Anderson and Co (1987b), Public hospital performance assessment - supplementary report, Wellington.

Ashton, T. (1999), Redesigning the welfare state in New Zealand: problems, policies, prospects, Oxford University Press, Auckland, chapter 10: The health reforms: to market and back?, pp. 134-153.

Bai, C. E. (1998), 'Introduction - the soft budget constraint and transition economies', Journal of Comparative Economics 26(1), 9-10.

Bai, C. E., Li, D. D., Tao, Z. \& Wang, Y. J. (2000), 'A multitask theory of state enterprise reform', Journal of Comparative Economics 28(4), 716-38. 
Bai, C. E. \& Wang, Y. J. (1998), 'Bureaucratic control and the soft budget constraint', Journal of Comparative Economics 26(1), 41-61.

Bernheim, B. D. \& Whinston, M. D. (1998), 'Incomplete contracts and strategic ambiguity', American Economic Review 88(4), 902-32.

Boston, J., Dalziel, P. \& St John, S. (1999), Redesigning the welfare state in New Zealand: problems, policies, prospects, Oxford University Press, Auckland.

Calcott, P. (2004), 'Government warnings and the information provided by safety regulation', International Review of Law and Economics 24, 71-88.

Clark, M. J. \& Randal, J. (2004), A first course in applied statistics, 1 edn, Pearson Education New Zealand, Auckland.

Coast Health Care Limited (1994), 'Annual report 1993-1994'.

Controller and Auditor General (1998), Report of the Controller and Auditor General: second report for 1998, Wellington.

Crawford, V. P. \& Sobel, J. (1982), 'Strategic information transmission', Econometrica $50(6), 1431-51$.

Crown Company Monitoring Advisory Unit (1996), Crown Health Enterprises - briefing to the incoming minister, CCMAU, Wellington.

Crown Company Monitoring Advisory Unit (1997), CHE comparative performance report for the fifteen months ended 30 September 1997, CCMAU, Wellington.

Davies, P., Ashton, T. \& Brown, P. (2001), From market power to people power: new directions in health reform in New Zealand, 3rd European Conference on Health Economics, York.

de Boer, M. (2000), WIES5A methodology and casemix purchase unit allocation for the 2000/2001 financial year. Frozen Specification for implementation on NMDS. 26 May 2000, Wellington. 
Deloitte, Touche \& Tohmatsu (1996), Trends in Area Health Board/CHE Performance, Crown Company Monitoring Advisory Unit, Wellington.

Deloitte, Touche \& Tohmatsu (1998), Booking System Implementation Review, Wellington.

Dewatripont, M. \& Maskin, E. S. (1995), 'Credit and efficiency in centralized and decentralized economies', The Review of Economic Studies 62(4), 541-555.

Donelan, K., Blendon, R. J., Schoen, C., Davis, K. \& Binns, K. (1999), 'The cost of health system change: public discontent in five nations', Health Affairs 18(2), 206-16.

Dur, R. \& Swank, O. H. (2005), 'Producing and manipulating information', Economic Journal 115(January), 185-99.

Easton, B. (1997), The commercialisation of New Zealand, University of Auckland Press, Auckland.

Evans, R. (1984), Strained mercy: The economics of Canadian health care, Butterworks, Toronto.

Farrell, J. (1993), 'Meaning and credibility in cheap-talk games', Games and Economic Behavior 5(4), 514-31.

Farrell, J. \& Gibbons, R. (1989), 'Cheap talk can matter in bargaining', Journal of Economic Theory.

Farrell, J. \& Rabin, M. (1996), 'Cheap talk', Journal of Economic Perspectives 10(3), 10318.

Fehr, E. \& Feld, A. (2002), Psychological foundations of incentives, IZA Discussion Paper 507, University of Zurich, Bonn.

Fielden, J., Cumming, J., Horne, J., Devane, P., Slack, A. \& Gallagher, L. (2005), 'Waiting for hip arthroplasty: economic costs and health outcomes', The Journal of Arthroplasty 20(8), 990-97. 
Fraser, G., Alley, P. \& Morris, R. (1993), Waiting Lists and Waiting Times: Their Nature and Management, National Advisory Committee on Core Health and Disability Support Services, Wellington.

George, D., Inglis, M. \& Rippin, S. (1997), Improving the performance of CHE Chief Executives, Study commissioned for CCMAU, Wellington.

Gibbons, R. (1992), A primer in game theory, Harvester Wheatsheaf, Hertfordshire.

Gilling, N. (1996), 'Resignation 'shows health reform failure", The Press 4 July (ed. 2), 6.

Gneezy, U. \& Rustichini, A. (2000), 'Pay enough or don’t pay at all', Quarterly Journal of Economics 115(3), 791-810.

Grossman, S. J. \& Hart, O. D. (1986), 'The costs and benefits of ownership: A theory of vertical and lateral integration', The Journal of Political Economy 94(4), 691-719.

Gujarati, D. (1995), Basic econometrics, 3rd edn, McGraw Hill, New York.

Guyan, C. (1998), 'Capital CHE cuts costs by $\$ 33 \mathrm{~m}$ ', The Evening Post 2 April (Edition 1), 1.

Hadorn, D. \& Holmes, A. (1997a), 'The New Zealand priority criteria project. part 1: Overview', British Medical Journal 314(7074), 131-34.

Hadorn, D. \& Holmes, A. (1997b), 'The New Zealand priority criteria project. part 2: coronary artery bypass graft surgery', British Medical Journal 314(7074), 135-38.

Harris, J. E. (1977), 'The internal organization of hospitals: Some economic implications', The Bell Journal of Economics 8(2), 467-482.

Hart, O. (1989), 'An economist's perspective on the theory of the firm', Columbia Law Review 89(7), 1757-1774.

Hart, O. D. \& Moore, J. (1985), Incomplete contracts and renegotiation, London School of Economics, London. 
Health and Disability Commissioner (1998), Canterbury Health Limited, Auckland.

Healthlink South (1996), 'Annual report'.

Holmström, B. (1982), 'Moral hazard in teams', Bell Journal of Economics 13(2), 324-40.

Holmström, B. \& Milgrom, P. (1991), 'Multitask principal-agent analyses: incentive contracts, asset ownership and job design', Journal of Law, Economics, and Organization $7,24-52$.

Holmström, B. \& Milgrom, P. (1994), 'The firm as an incentive system', American Economic Review 84(4), 972-91.

Hornblow, A. \& Barnett, P. (2000), 'A turbulent decade: lessons from the "health reforms"', New Zealand Medical Journal 113, 133-34.

Hospital and Related Services Taskforce (1988), Unshackling the hospitals, Government Printing Office, Wellington.

Howell, B. (2000), 'Ownership-based analysis of public hospital corporatisation in New Zealand', Agenda 7(3), 237-250.

James, H. S. (2003), Why does the introduction of monetary compensation produce a reduction in performance?, Contracting and Organizations Research Institute Working Paper, Columbia.

Kisely, S. (1997), 'Working in New Zealand', British Medical Journal 315(7105), 2.

Kornai, J. (1980), Economics of shortage, North-Holland, Amsterdam.

Kornai, J. (1986), 'The soft budget constraint', Kyklos 46(3), 13-30.

Kornai, J. (1998), 'The place of the soft budget constraint syndrome in economic theory', Journal of Comparative Economics 26(1), 11-17.

Kornai, J. (2001), 'Hardening the budget constraint: the experience of the post-socialist countries', European Economic Review 45(9), 1573-99. 
Kreps, D. M. (1997), 'Interaction between norms and economic incentives: intrinsic motivation and extrinsic incentives', Journal of Finance 87(2), 359-64.

Kreps, D. M. \& Sobel, J. (1994), Handbook of game theory with economic applications. Edited by R. Aumann and H. Sergiu, Elsevier Science, Amsterdam, chapter 25: Signalling.

Labour Party (1999), Focus on patients: Labour on health, New Zealand Labour Party, Wellington.

Lazear, E. P. (1989), 'Pay equality and industrial politics', Journal of Political Economy $97(3), 561-80$.

Maskin, E. S. \& Tirole, J. (2004), 'The politician and the judge: accountability in government', The American Economic Review 94(4), 1034-1054.

Maskin, E. S. \& Xu, G. (2001), 'Soft budget constraint theories - from centralization to the market', Economics of transition 9(1), 1-27.

Matthews, S. A., Okuno-Fujiwara, M. \& Postlewaite, A. (1991), 'Refining cheap talk equilibria', Journal of Economic Theory 55(2), 247-73.

Medical Council of New Zealand (1994), Doctors' duties in an environment of competition or resource limitation, Wellington.

Medical Council of New Zealand (2000), Guidelines on Responsibilities of Doctors in Management and Governance, Wellington.

Medical Council of New Zealand (updated October 1999), Ethical guidelines for doctors' duties in an environment of competition or resource limitation, Wellington.

Ministry of Health (1995), Acute medical volumes: trends 1988/89 - 1994/95, Ministry of Health, Wellington.

Ministry of Health (1996), Selected morbidity and hospital data 1995/96, Ministry of Health, Wellington. 
Ministry of Health (1998), Public Perceptions of the New Zealand Health System, Ministry of Health, Wellington.

Ministry of Health (1999), Hospital Throughput 1997/98, Ministry of Health, Wellington.

Ministry of Health (2000), Hospital Throughput 1998/99, Ministry of Health, Wellington.

Ministry of Health (2001a), Health Expenditure Trends in New Zealand 1980-2000, Ministry of Health, Wellington.

Ministry of Health (2001b), Hospital Throughput 1999/2000, Ministry of Health, Wellington.

Ministry of Health (2005), Health Expenditure Trends in New Zealand 1992-2003, Ministry of Health, Wellington.

Mold, F. (1996), 'CHE reaps $\$ 23 \mathrm{~m}$ from cost cuts', Waikato Times 18 Sept (Edition 2), 1.

Myerson, R. (1989), 'Credible negotiation statements and coherent plans', Journal of Economic Theory 48(1), 264-303.

Myerson, R. (2008), The New Palgrave Dictionary of Economics, second edn, Macmillan, London, chapter 3016: Revelation principle.

Pauly, M. V. (1980), Doctors and their workshops, University of Chicago Press, Chicago.

Rains, M., Hogan, J. \& de Boer, M. (2001), WIES8A methodology and casemix purchase unit allocation for the 2001/2002 financial year. Frozen Specification for implementation on NMDS. 9 May 2001 with minor modifications on 3 August and 10 October 2001, Wellington.

Schmidt, K. M. (1996), 'The costs and benefits of privatization: an incomplete contracts approach', Journal of Law, Economics, and Organization 12(1), 1-24.

Simon, H. A. (1951), 'A formal theory of the employment relationship', Econometrica $19(3), 293-305$. 
Spence, A. M. (1973), 'Job market signalling', Quarterly Journal of Economics 87(3), 35574.

Steinwald, B. \& Dummit, L. (1989), 'Hospital case-mix change: sicker patients or drg creep', Health Affairs 8(2), 35-47.

Stewart Lawrence, M. A. \& Lowe, T. (1994), 'the great experiment: financial management reform in the NZ health sector', Accounting, Auditing 85 Accountability Journal 7, 6895.

Tirole, J. (1986), 'Hierachies and bureaucracies: on the role of collusion in organizations', Journal of Law, Economics, and Organization 2(2), 181-214.

Tirole, J. (1999), 'Incomplete contracts: where do we stand?', Econometrica 67(4), 74181.

Troughton, P. (1993), The creation of New Zealand's Crown Health Enterprises, Crown Health Enterprise Establishment Unit, Wellington.

Upton, S. (1991), Your Health and the Public Health: A Statement of Government Health Policy, Wellington.

Vaithianathan, R. (1999a), Economic incentives and clinical decisions, PhD thesis, Economics Department, University of Auckland, Auckland.

Vaithianathan, R. (1999b), 'The failure of corporatisation: public hospitals in New Zealand', Agenda 6(4), 325-38.

Varian, H. (1992), Microeconomic analysis, W. W. Norton \& Company, New York.

Williams, C. (1996), 'Doctors warned over New Zealand jobs', British Medical Journal 313(7057), 578.

Williamson, O. (1985), The economic institutions of capitalism: firms, markets, relational contracting, Free Press, New York. 


\title{
Glossary
}

\section{Health sector-related acronyms}

\author{
AHB Area Health Board \\ CHE Crown Health Enterprise \\ DRG Diagnostic Related Grouping \\ HHS Hospital and Health Service \\ ICD International Classification of Diseases \\ MDC Major Diagnostic Category \\ $\mathrm{MoH} \quad$ Ministry of Health \\ NMDS National Minimum Data Set \\ NZHIS New Zealand Health Information Service
}

\section{General modelling terminology}

The actors are assigned genders to allow the use of personal pronouns where they can be used unambiguously. The genders are noted in brackets below.
F $\quad$ Funder (male)
A Administrator (female)
$S \quad$ Specialist (male)
C Consumer (female)
BNE Bayesian Nash equilibrium
NPE Neologism-proof Bayesian Nash equilibrium
PBE Perfect Bayesian Nash equilibrium 


\section{Chapter-specific modelling terminology}

\section{Chapter 3}

$\begin{array}{cl}\text { FBC } & \text { Flexible budget constraint } \\ \text { HBC } & \text { Hard budget constraint } \\ \text { SBC } & \text { Soft budget constraint } \\ b_{t} & \text { The budget the funder sets in stage } t \\ c \sim G_{e_{1}} & \text { The organisation's baseline costs (determined in stage 1) } \\ \kappa & \text { The organisation's ultimate costs (determined in stage } 2) \\ e_{t} & \text { The administrator's effort in stage } t . \\ w_{1 M} & \text { A bonus that } A \text { is eligible for if she meets the original budget target. } \\ w_{1 N} & \text { A bonus that } A \text { is eligible for if she does not meet the original budget target. } \\ w_{2} & \text { A bonus that } A \text { is eligible for if she meets a revised budget target. } \\ \omega & \text { The administrator's ultimate bonus payment }\end{array}$

\section{Chapter 4}

$t \in\left\{t_{1}, t_{2}, t_{3}\right\} \quad$ A treatment authorised by the administrator

$T \quad$ The set of possible treatments

$T_{0} \quad$ The menu of treatments $S$ could convince $A$ to authorise

$C(t) \quad$ The cost of performing a treatment $t$

$\delta(t) \quad$ The damage from an adverse event following treatment $t$

$\pi \sim H \quad$ Probability that a patient suffers an adverse event; $\pi \in[0,1]=\Pi$

$\mu(\pi) \quad$ The specialist's message strategy

$\tau(\mu) \quad$ The administrators's treatment strategy 


\section{Chapter 5}

$e \quad$ An action that $A$ may carry out that increases the likelihood of high quality

$m_{i} \quad$ A message, or signal, about $q_{i}$, where a message can indicate quality is low $\left(m \in M_{L}\right)$ or high $\left(m \in M_{H}\right)$

$q_{i} \quad$ A state nature of nature reflecting the effect, or quality, associated with a particular administrator, where quality can be low $\left(q_{L}\right)$ or high $\left(q_{H}\right)$

$q^{\prime} \quad$ Expected quality under a replacement administrator

$\pi \quad$ Probability of high quality

$\pi(e) \quad$ Probability of high quality for a given level of effort

$w \quad$ The specialist's wage, which is decided by the administrator

$w^{\prime} \quad$ The wage the specialist expected under a new administrator's regime

$\underline{w} \quad$ A wage threshold below which the specialist's message lacks credibility

$\bar{w} \quad$ A wage threshold above which the specialist's message lacks credibility

$W_{\text {on }} \quad$ A set of wages that, in equilibrium, permit the specialist to send a message that is credible to the consumer

$W_{\text {off }} \quad$ A set of wages that, in equilibrium, erodes the specialist's credibility with the consumer

$y \in\{R, T\} \quad$ The consumer's vote to reappoint or terminate the administrator's tenure $Y \quad$ The set of possible voting outcomes over the administrator's tenure

$Y_{0} \quad$ The set of tenure decisions that the consumer could be persuaded to make 
Appendices 


\section{A.1 Appendices to Chapter 2}

\section{A.1.1 Weighted Inlier Equivalent Separations methodology}

This appendix discusses the Weighted Inlier Equivalent Separations (WIES) methodology that underpins the calculation of the cost weight variable used in the empirical analysis. The case-mix methodology used to calculate cost weights was introduced to New Zealand from Victoria, Australia in 1998. The methodology can be applied to administrative inpatient data collected from 1988.

\section{Method}

For a given year, the cost weight index for all public inpatient admissions is normalised to a value of 1.0. That is, a case of average complexity with an average length of stay would be given a cost weight of 1.0. Less complex cases attract a lower cost weight and cases that are more complex or involve longer-than-average stays, but are otherwise the same, attract a higher cost weight.

Cost weights are generated for each inpatient event in five steps.

1. Hospitals provide patient event information to NZHIS.

2. The NZHIS allocates the event a Diagnostic Related Group (DRG) based on the diagnosis and treatment information in the record.$^{1}$

3. The patient's length of stay (LOS) is calculated and this is used to determine whether their stay is a low outlier, an inlier (ie. an average length of stay), or a high outlier.

4. The DRG and LOS combination is matched to a cost weight from the Victorian DRG cost weight schedule.

\footnotetext{
${ }^{1}$ Diagnostic Related Group (DRG) codes categorise discharges based on the diagnosis made and procedures undertaken (Ministry of Health 2001b). This analysis uses version 3.1 of the Australian National Diagnosis Related Groups (AN-DRG) system.
} 
5. The final step is to adjust the WIES cost weight to include a co-payment if mechanical ventilation was required.

\section{Changes to the WIES methodology and coding behaviour}

Since being introduced to New Zealand this methodology has been updated several times. This research uses the first version introduced at the end of the reform period, Version 5 with Amendments for New Zealand (WIES5A) (de Boer 2000).

Cost weights were constructed for each discharge record specifically for this research. This provides a single, consistent methodology and set of reference variables for all years of data in the data set. The cost weights for all years are based on a single Victorian DRG cost weight reference table for 1999. This approach gives the cost per case in real, inflation-free terms. As such, the cost weights do not change over time due to changes in the costs of treating a particular DRG due to health service price inflation. Another, but trivial, implication is that the index is equal to 1.0 for the 1999 year only. We normalise the cost weights in all other years to $19992^{2}$

The NZHIS began applying cost weights to discharge records in 1998. Therefore, no single cost weight methodology is appropriate for the entire period of analysis from 1988 to 1999. Our empirical analysis uses the WIES5A cost weight methodology, the earliest version that the NZHIS used, to calculate a consistent series of cost weights for the entire period.

This approach has some limitations primarily related to concern around coding consistency and changes to the coding practices (Ministry of Health 1996). In particular, the WIES5A cost weight methodology assumes that records are generated using the Australian coding rules (AN-DRG 3.1). These rules were not used in New Zealand prior to 1

\footnotetext{
${ }^{2}$ The average cost weight for the complete NMDS is 1.0. However, this research focuses on publiclyfunded discharges and some additional records in the complete NMDS have been excluded for consistency purposes; these exclusions are discussed below. The excluded cases mean that the average cost weight in 1999 for the analytical data set used in this research is not equal to 1.0. This does not have a material impact on our analysis as the cost weights form a cost index, and the absolute values are not the focus of the analysis.
} 
July 1995. Prior to this time fewer diagnoses were reported per discharge record, which may alter the DRG to which a patient's treatment is assigned in turn affecting the cost weight. Under-reporting tends to lower the DRG assigned to an event, in turn resulting in a lower cost weight being assigned than should be if the discharge record were completed. While reporting behaviour may change after July 1995, reporting behaviour from before the CHE reforms up to July 1995 is likely to be relatively consistent.

\section{'Waiting list' funding injections}

The government injected extra funding into the public health system to address concerns over long waiting times on surgical priority lists during 1995 and again in 1997!3 The funding injections allowed people with less severe conditions to enter the hospital system and reduced waiting times, which has been shown to be associated with lower economic costs for some conditions (Fielden et al. 2005). As well as changing the health status profile and cost of treating the average patient, the funding injections were associated with a change in the proportion of operations performed in public and private facilities.

Therefore, the main analysis takes a conservative approach and concentrates on data from 1 July 1988 to 30 June 1995. Section 2.5.2 in Chapter 2 reports on supplementary analyses using data beyond 1 July 1995; analyses including the additional data from 1 July 1995 are specifically noted. The coding changes and the funding injections noted above mean the results of these supplementary analyses should be read with caution. In particular, the coding changes are expected to result in higher reported costs beyond 1995. This change might therefore provide spurious support for the hypothesis about the effects of the CHE reforms on costs, where the effect of the coding changes are not statistically controlled for. Equivalently, the funding injections could potentially lower the average cost weight by allowing access to lower priority patients. Such a change could disguise the effect of the CHE reforms on costs.

\footnotetext{
$3 \$ 20$ million was injected into the health budget as of July 1995 to fund extra operations, which was equivalent to around $0.5 \%$ of the public funding to public and private hospitals.
} 


\section{A.1.2 NMDS records excluded from analytical data set}

This appendix discusses NMDS records excluded from the analytical data set, which were summarised in Section 2.3.1. We list the main types of exclusions below.

1. Non-treated admissions, such as boarders who accompany patients receiving treatment but who are not treated themselves, or people who are admitted and then have their operation cancelled.

2. Error DRGs (AN-DRGs 951, 952, 955 and 956) that indicate invalid or missing diagnosis data. These codes are discussed below.

3. Procedures that are excluded from case-mix purchasing (brief or same day procedures including renal dialysis and some chemotherapy, radiotherapy, sleep apnoea, lithotripsy, colposcopies, cystoscopies, endoscopic retrograde cholangiopancreatography, colonoscopies, gastroscopies, bronchoscopies and blood transfusion services).

4. 'Well babies' due to variation in recording practices across hospitals.

\section{Cases with zero cost weights}

The AN-DRG method may allocate a case a zero cost weight where there is insufficient or incorrect information. For example, error DRGs may be applied where insufficient or contradictory information is provided, and a zero cost weight is assigned to such cases. This analysis excludes cases that are assigned one of four error DRGs: AN-DRGs 951, 952, 955, and 956.4 Including records with a zero cost weight would create a downward bias in the data. Excluding these records is a conservative approach, as 68.7 percent of the cases with zero cost weights fall in the period before the reforms. Including these cases would increase the likelihood of showing a specious, but statistically significant, increase in costs after the reforms.

\footnotetext{
${ }^{4}$ Two of the error codes relate to maternity diagnoses. AN-DRG 951 is used for unacceptable obstetric diagnosis. AN-DRG 955 is used for a neonatal diagnosis not consistent with age or weight. The other two error codes are general recording error codes. AN-DRG 952 is applied to discharges with invalid data. AN-DRG 956 is used where records have valid diagnoses but which are not acceptable as a principal diagnosis (reason for admission).
} 


\section{Publicly-funded, privately-performed procedures}

Publicly-funded, privately-performed procedures are excluded due to both theoretical and empirical concerns. A priori, it is unclear that the CHE reforms would have had the same effect on the relationship between medical staff and private hospital managers as the private hospitals were for-profit organisations or were non-profit but run by private interests. As private hospitals were not directly subject to the CHE reforms, the reforms are unlikely to have directly affected the relations between private hospital managers and clinicians. Despite this independence from the CHE reforms, however, the CHE reforms may have altered the contracting processes and incentives between the public purchasing agent and private provider. The objective of this analysis is not to examine alternative purchasing models, such as a split between a public purchaser and private provider. Rather we focus on the effect of changing incentives within a complex organisational structure.

Furthermore, there are differences between the patient populations receiving publiclyfunded treatment in public or private hospitals. For example, the demographic characteristics of patients and types of publicly-funded procedures performed in private facilities differed significantly from those in public facilities. Unobserved variables that explain these different characteristics may make it difficult to identify any independent relationship between the reforms and costs. The theoretical concerns and empirical differences between the publicly and privately-performed procedures raise significant doubts about the relevance of including, and validity of comparing, privately-performed procedures with publicly-performed procedures.

The appendix table below reproduces Table A2.1 of the Ministry of Health (2000) report. A subset of the Ministry's exclusions were relevant to our study, so the total number of exclusions in Table 2.1 is greater than the exclusions from our analytical data set. 


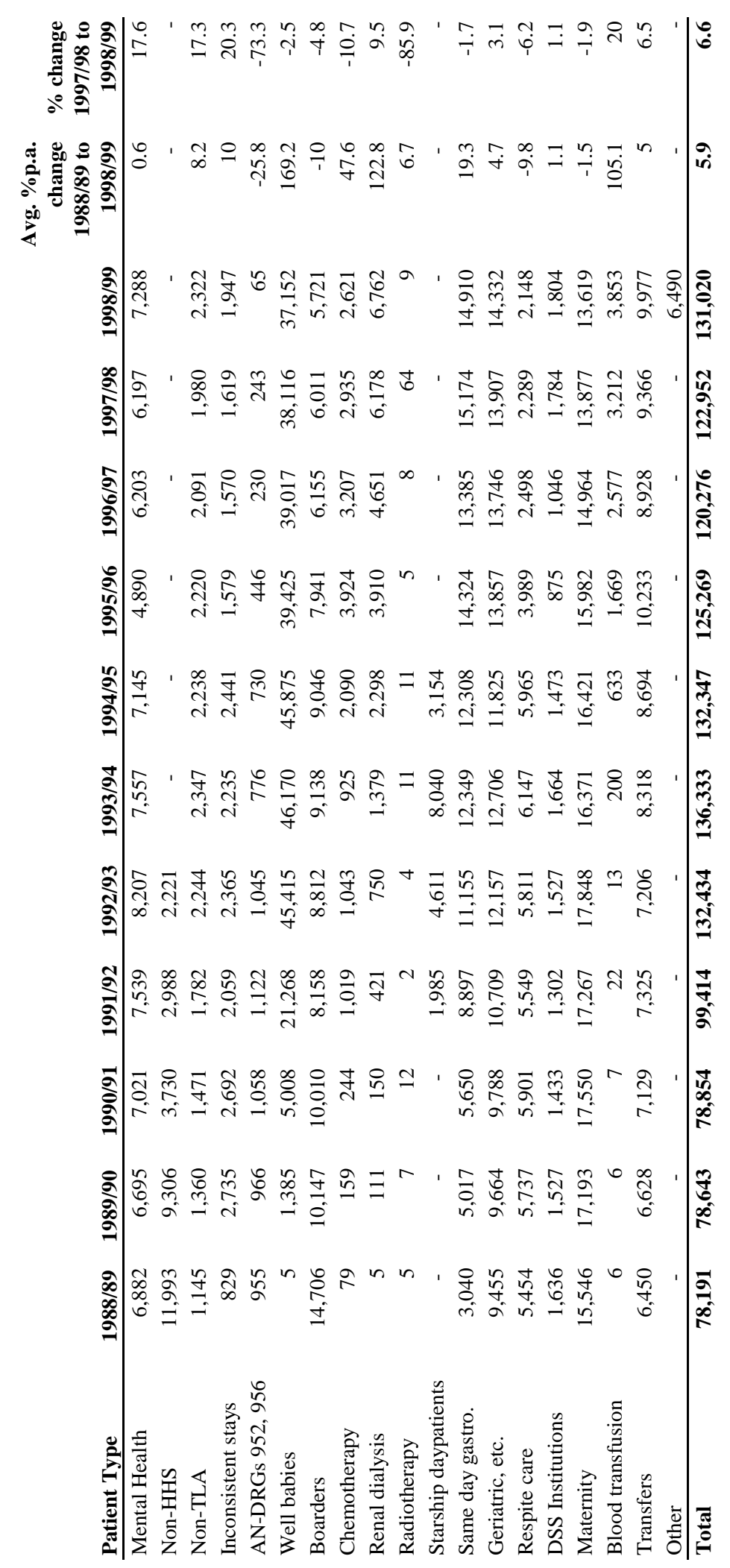

Table A.1: Records excluded by the filtering process - 1988/89 to 1998/99 


\section{A.1.3 Tabulation of regression statistics}

This appendix expands on Table 2.8 on page 36 in Chapter 2, Table A.2 adds the standard errors (s.e.) and p-values of the analysis' main regression models.

\begin{tabular}{|c|c|c|c|c|c|c|c|c|c|c|}
\hline Model & Intercept & Period & Age & $\begin{array}{c}\text { Period } \\
\text { x Age }\end{array}$ & Age $^{2}$ & $\begin{array}{c}\text { Period } \\
\text { x Age }^{2}\end{array}$ & Month & $\begin{array}{c}\text { Period } \\
\text { x Month }\end{array}$ & Month $^{2}$ & $\begin{array}{c}\text { Period } \\
\text { x Month }^{2}\end{array}$ \\
\hline 1 & 0.7768 & & 0.0034 & & 0.00021 & & & & & \\
s.e. & 0.0052 & & 0.0003 & & 0.000003 & & & & & \\
p & $<0.001$ & & $<0.001$ & & $<0.001$ & & & & & \\
\hline 2 & 0.8758 & -0.256 & 0.0023 & & 0.00023 & & & & & \\
s.e. & 0.0055 & 0.0048 & 0.0003 & & 0.000003 & & & & & \\
p & $<0.001$ & $<0.001$ & $<0.001$ & & $<0.001$ & & & & & \\
\hline 3 & 0.8626 & -0.2179 & -0.0005 & 0.009 & 0.00028 & -0.00018 & & & & \\
s.e. & 0.0066 & 0.0106 & 0.0004 & 0.0006 & 0.000004 & 0.00001 & & & & \\
p & $<0.001$ & $<0.001$ & 0.1949 & $<0.001$ & $<0.001$ & $<0.001$ & & & & \\
\hline 4 & 1.1069 & -0.3369 & -0.0017 & 0.0103 & 0.00029 & -0.00019 & -0.007 & 0.0053 & & \\
s.e. & 0.0077 & 0.0382 & 0.0003 & 0.0006 & 0.000004 & 0.00001 & 0.0001 & 0.0005 & & \\
p & $<0.001$ & $<0.001$ & $<0.001$ & $<0.001$ & $<0.001$ & $<0.001$ & $<0.001$ & $<0.001$ & & \\
\hline 5 & 1.1074 & 1.849 & -0.0017 & 0.0103 & 0.00029 & -0.00019 & -0.0071 & -0.0555 & 0.000001 & 0.0004 \\
s.e. & 0.0096 & 0.4237 & 0.0003 & 0.0006 & 0.000004 & 0.00001 & 0.0006 & 0.0118 & 0.000009 & 0.0001 \\
p & $<0.001$ & $<0.001$ & $<0.001$ & $<0.001$ & $<0.001$ & $<0.001$ & $<0.001$ & $<0.001$ & 0.927 & $<0.001$ \\
\hline 6 & -0.2552 & -0.0886 & 0.0016 & 0.0019 & 0.000043 & -0.000026 & -0.0015 & 0.0013 & & \\
s.e. & 0.0007 & 0.0036 & 0.00003 & 0.0001 & 0.0000004 & 0.0000006 & 0.00001 & 0 & & \\
p & $<0.001$ & $<0.001$ & $<0.001$ & $<0.001$ & $<0.001$ & $<0.001$ & $<0.001$ & $<0.001$ & & \\
\hline 7 & -0.2561 & 0.2171 & 0.0016 & 0.0019 & 0.000043 & -0.000026 & -0.0015 & -0.0073 & -0.000001 & 0.00006 \\
s.e. & 0.0009 & 0.0397 & 0.00003 & 0.0001 & 0.0000004 & 0.0000006 & 0.0001 & 0.0011 & 0.000001 & 0.000008 \\
p & $<0.001$ & $<0.001$ & $<0.001$ & $<0.001$ & $<0.001$ & $<0.001$ & $<0.001$ & $<0.001$ & 0.1151 & $<0.001$ \\
\hline
\end{tabular}

Table A.2: Summary of regression model coefficients and statistics 


\section{A.1.4 Supplementary analysis excluding outliers}

This appendix reports on a supplementary analysis to Chapter 2 that excludes cost weight outliers.

A standard outlier test identified that cases with a cost weight greater than 2.13 were outliers (Clark \& Randal 2004). The test determined that 727,997 cases (11.0 percent) were outliers. To examine the effects of the outliers on the estimated relationships, the models specified in Table 2.3 were re-estimated using a data set excluding outliers cases. Table A.3 reports the supplementary regression results, and is comparable to Table 2.8 on page 36 .

\begin{tabular}{|c|c|c|c|c|c|c|c|c|c|c|}
\hline $\begin{array}{c}\text { Model } \\
\text { (Coefficient) }\end{array}$ & $\begin{array}{c}\text { Intercept } \\
\left(\alpha_{0}\right)\end{array}$ & $\begin{array}{c}\text { Period } \\
\left(\beta_{0}\right)\end{array}$ & $\begin{array}{c}\text { Age } \\
\left(\alpha_{1}\right)\end{array}$ & $\begin{array}{c}\text { Period } \\
\text { x Age } \\
\left(\beta_{1}\right)\end{array}$ & $\begin{array}{c}\text { Age }{ }^{2} \\
\left(\alpha_{2}\right)\end{array}$ & $\begin{array}{c}\text { Period } \\
x_{\text {Age }}^{2} \\
\left(\beta_{2}\right)\end{array}$ & $\begin{array}{c}\text { Month } \\
\left(\alpha_{3}\right)\end{array}$ & $\begin{array}{c}\text { Period } \\
\text { x Month } \\
\left(\beta_{3}\right)\end{array}$ & $\begin{array}{c}\text { Month }{ }^{2} \\
\left(\alpha_{4}\right)\end{array}$ & $\begin{array}{c}\text { Period } \\
\text { x Month } \\
\left(\beta_{4}\right)\end{array}$ \\
\hline 1 & $0.4647 \ddagger$ & & $0.0039 \ddagger$ & & $0.00002 \ddagger$ & & & & & \\
\hline 2 & $0.4769 \ddagger$ & $-0.0312 \ddagger$ & $0.0038 \ddagger$ & & $0.00002 \ddagger$ & & & & & \\
\hline 3 & $0.4817 \ddagger$ & $-0.0430 \ddagger$ & 0.0033 & $0.0014 \ddagger$ & $0.00003 \ddagger$ & $-0.00002 \ddagger$ & & & & \\
\hline 4 & $0.5253 \ddagger$ & $-0.0743 \ddagger$ & $0.0031 \ddagger$ & $0.0016 \ddagger$ & $0.00003 \ddagger$ & $-0.00002 \ddagger$ & $-0.0013 \ddagger$ & $0.0011 \ddagger$ & & \\
\hline 5 & $0.5241 \ddagger$ & $0.1756 \ddagger$ & $0.0030 \ddagger$ & $0.0016 \ddagger$ & $0.00003 \ddagger$ & $-0.00002 \ddagger$ & $-0.0011 \ddagger$ & $-0.0060 \ddagger$ & -0.000002 & $0.00005 \ddagger$ \\
\hline 6 & $-0.3359 \ddagger$ & $-0.0456 \ddagger$ & $0.0019 \ddagger$ & $0.0011 \ddagger$ & $0.00002 \ddagger$ & $-0.00001 \ddagger$ & $-0.0009 \ddagger$ & $0.0006 \ddagger$ & & \\
\hline 7 & $-0.3370 \ddagger$ & $0.1330 \ddagger$ & $0.0019 \ddagger$ & $0.0011 \ddagger$ & $0.00002 \ddagger$ & $-0.00001 \ddagger$ & $-0.0008 \ddagger$ & $-0.0044 \ddagger$ & -0.000002 & $0.00004 \ddagger$ \\
\hline
\end{tabular}

$¥$ significant at an $\alpha<0.01 \%$ level

Table A.3: Summary of supplementary regression model results, excluding outliers

Table A.3 indicates that excluding outlier cases did not have a statistically significant effect on the main qualitative results related to the period variables. However, the higher cost outlier cases were mainly associated with younger and older people, although the highest cost cases were for 25-29 year olds with mental health issues, as shown in Figures A.1 and A.3 below. As a result, removing these cases changed the algebraic signs of the estimated coefficients for the age variable in some models. In essence, excluding the outlier cases disproportionately censors cases at very young ages, and this could cloud or hide the expected quadratic relationship between age and cost weight. 
Figures A.1 and A.3 show the simple relationship between age and cost weight for inlier and outlier cases. For presentation purposes, the figures use the average cost weight per age group. The records are grouped into 5-year bands, except for the following age groups: under 1 year old, 1 up to but not including 5 year olds, and 90 years old plus.

Although only just over 4.8 percent of the cases in the under-one year old age group are outliers, these cases account for just under 41.6 percent of the cost for this age group. The next two older age groups account for a substantially lower proportion of the age group costs. The outliers in the 5-9 and 10-14 year old age groups account for 2.7 percent and 3.9 percent of the cases, but 22.3 percent and 29.2 percent of the cost. By removing the very young, but disproportionately high cost outlier cases, this is likely to weaken or eliminate the expected non-linear relationship between age and cost weight. 


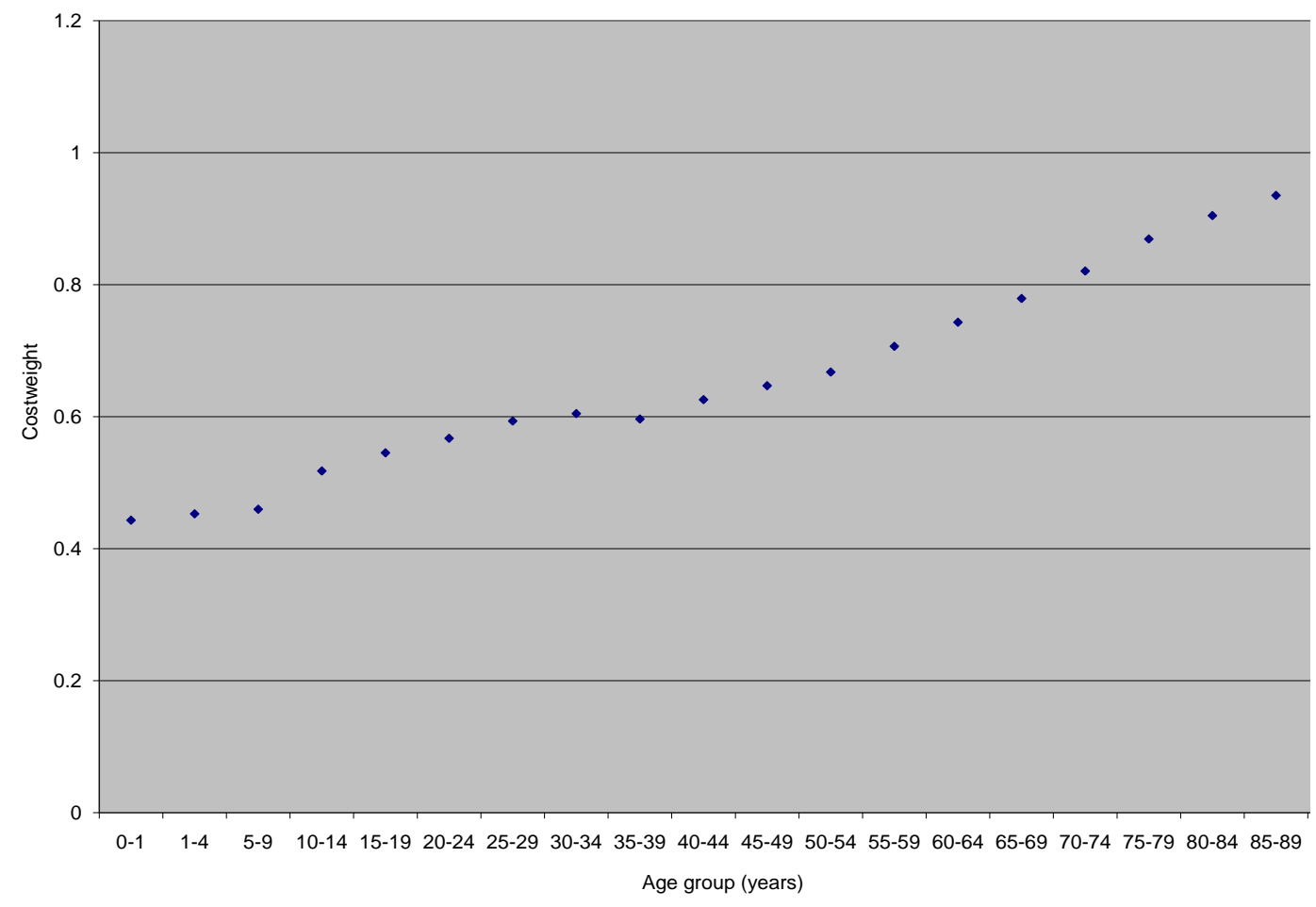

Figure A.1: Relationship between cost weight and age, for inlier cases

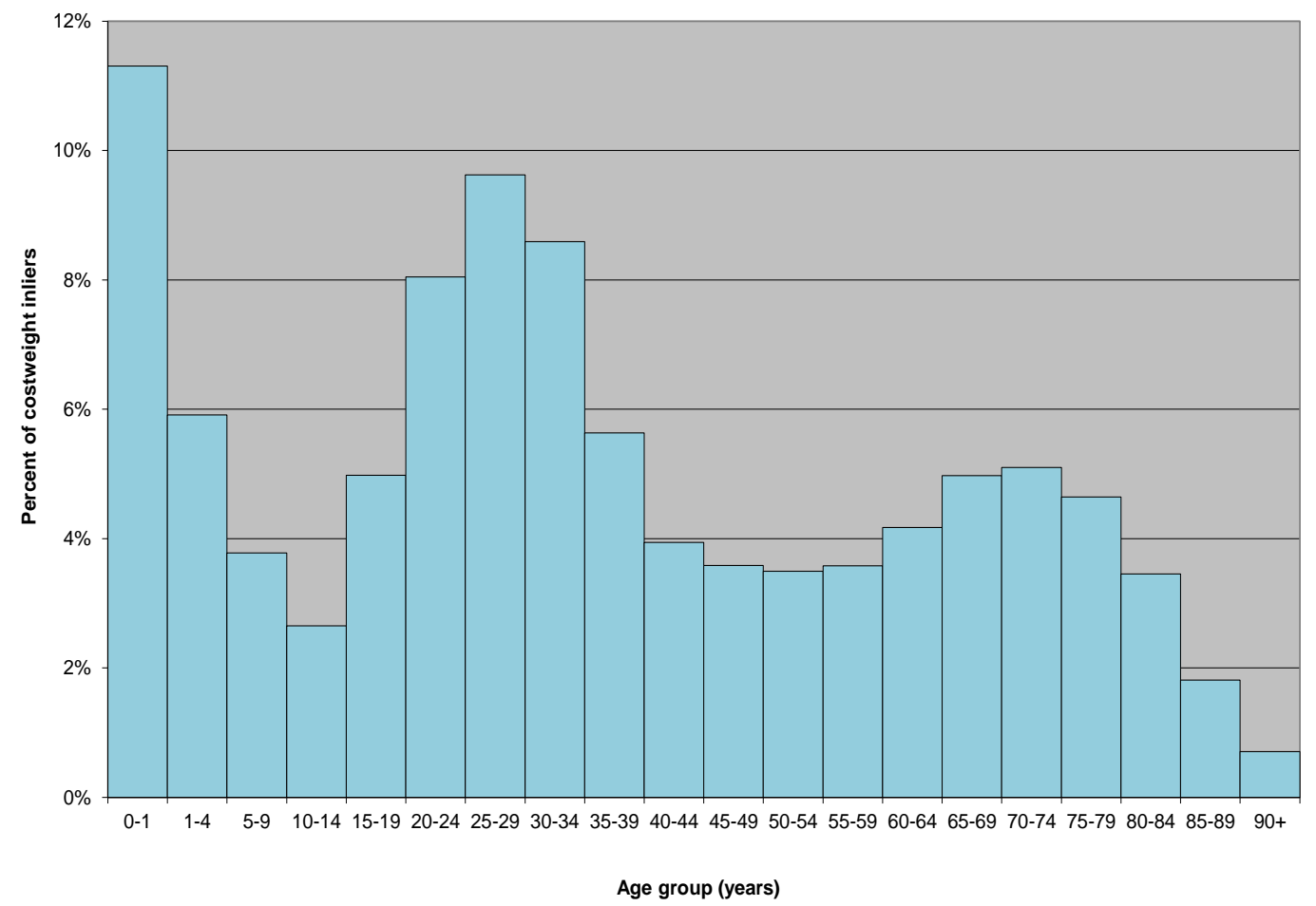

Figure A.2: Frequency of inlier cases by age 


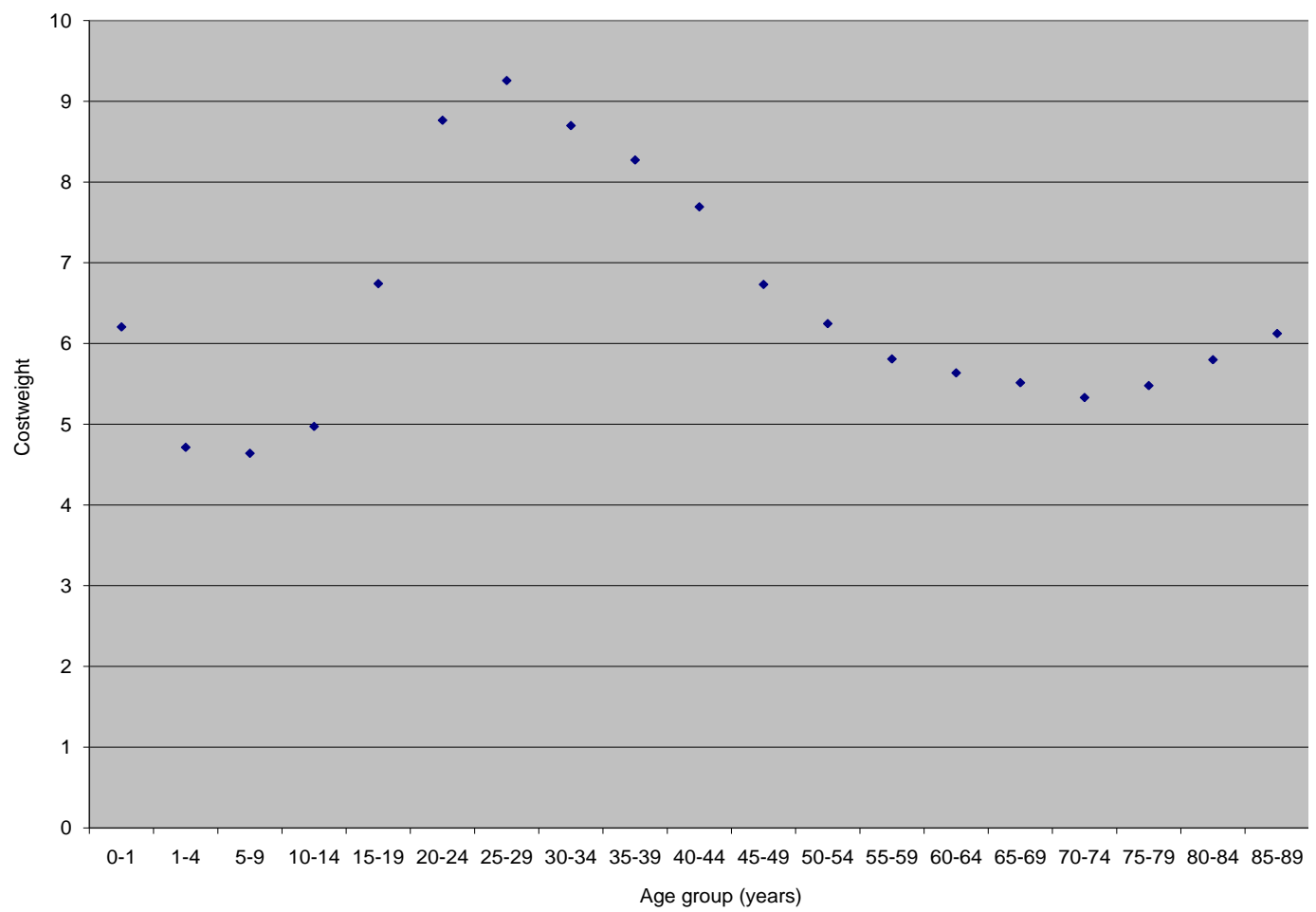

Figure A.3: Relationship between cost weight and age, for outlier cases

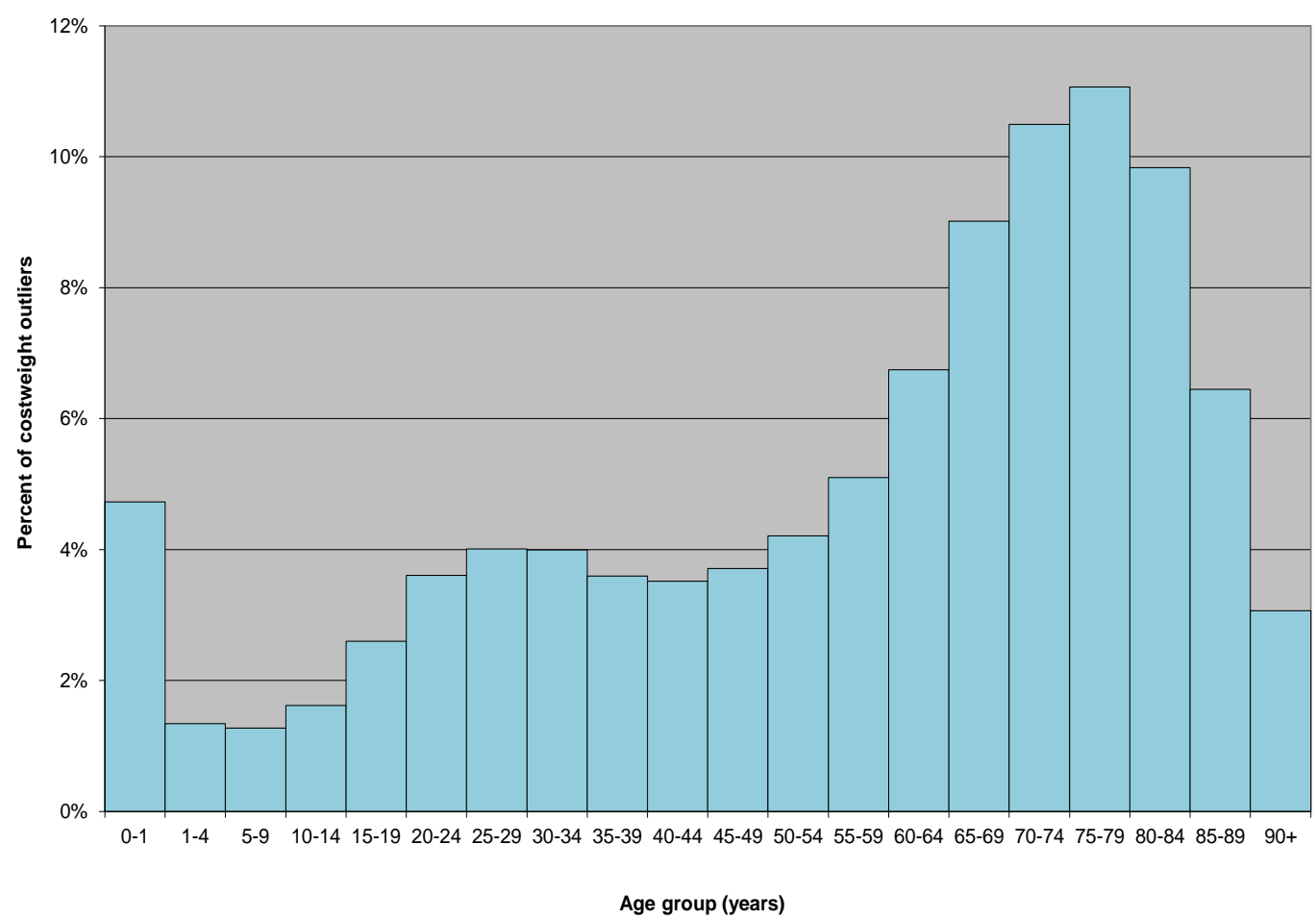

Figure A.4: Frequency of outlier cases by age 


\section{A.1.5 Supplementary analysis censoring transition period}

This appendix reports on a supplementary analysis to Chapter 2 that censors a 3 month or 6 month transitionary period following the reforms.

These models give statistically significant coefficients that indicate the reforms were associated with cost increases immediately after the reforms.

1 July 1993 to 30 September censored

\begin{tabular}{|c|c|c|c|c|c|c|c|c|c|c|}
\hline Model & Intercept & Period & Age & $\begin{array}{c}\text { Period } \\
\text { x Age }\end{array}$ & Age $^{2}$ & $\begin{array}{c}\text { Period } \\
\text { x Age }^{2}\end{array}$ & Month & $\begin{array}{c}\text { Period } \\
\text { x Month }\end{array}$ & Month $^{2}$ & $\begin{array}{c}\text { Period } \\
\text { x Month }^{2}\end{array}$ \\
\hline (Coefficient) & $\left(\alpha_{0}\right)$ & $\left(\beta_{0}\right)$ & $\left(\alpha_{1}\right)$ & $\left(\beta_{1}\right)$ & $\left(\alpha_{2}\right)$ & $\left(\beta_{2}\right)$ & $\left(\alpha_{3}\right)$ & $\left(\beta_{3}\right)$ & $\left(\alpha_{4}\right)$ & $\left(\beta_{4}\right)$ \\
\hline $4-3$ & $1.0882 \ddagger$ & $-0.3768 \ddagger$ & $-0.0023 \ddagger$ & $0.0101 \ddagger$ & $0.0003 \ddagger$ & $-0.0002 \ddagger$ & $-0.0067 \ddagger$ & $0.0058 \ddagger$ & & \\
\hline $5-3$ & $1.0857 \ddagger$ & $2.5442 \ddagger$ & $-0.0023 \ddagger$ & $0.0101 \ddagger$ & $0.0003 \ddagger$ & $-0.0002 \ddagger$ & $-0.0065 \ddagger$ & $-0.0738 \ddagger$ & -0.000004 & $0.0005 \ddagger$ \\
\hline
\end{tabular}

1 July 1993 to 30 December censored

\begin{tabular}{|c|c|c|c|c|c|c|c|c|c|c|}
\hline Model & Intercept & Period & Age & $\begin{array}{l}\text { Period } \\
\text { x Age }\end{array}$ & $\mathrm{Age}^{2}$ & $\begin{array}{l}\text { Period } \\
\mathrm{x} \mathrm{Age}^{2}\end{array}$ & Month & $\begin{array}{c}\text { Period } \\
\text { x Month }\end{array}$ & Month $^{2}$ & $\begin{array}{c}\text { Period } \\
\text { x Month }\end{array}$ \\
\hline (Coefficient) & $\left(\alpha_{0}\right)$ & $\left(\beta_{0}\right)$ & $\left(\alpha_{1}\right)$ & $\left(\beta_{1}\right)$ & $\left(\alpha_{2}\right)$ & $\left(\beta_{2}\right)$ & $\left(\alpha_{3}\right)$ & $\left(\beta_{3}\right)$ & $\left(\alpha_{4}\right)$ & $\left(\beta_{4}\right)$ \\
\hline 4-6 & $1.0882^{\ddagger}$ & $-0.5423^{\dagger}$ & $-0.0023 \ddagger$ & $0.0099 \ddagger$ & $0.0003 \ddagger$ & $-0.0002 \ddagger$ & $-0.0067 \ddagger$ & $0.008 \ddagger$ & & \\
\hline $5-6$ & $1.0857^{\ddagger}$ & $1.4715^{\ddagger}$ & $-0.0023 \ddagger$ & $0.0099 \ddagger$ & $0.0003 \ddagger$ & $-0.0002 \ddagger$ & $-0.0065^{\ddagger}$ & $-0.0457 \ddagger$ & -0.000004 & $0.0004^{\ddagger}$ \\
\hline
\end{tabular}

Table A.4: Summary of regression model variables using censored data

Models 4-3 and 5-3, which censor three months of data following the reforms, have similar patterns to the main models, 6 and 7. Models 4-6 and 5-6 censor six months of data following the reforms. Model 4-6 shows that the reforms were associated with a switch from a negative linear trend to a positive linear trend, as represented in Figure A.7; Model 5-6 also gives a similar pattern to model 7, as shown in Figure A.8.

These results do not provide strong evidence that there was a transition period before the full effects of the reforms manifested. Comparing Model 4-3 and Model 4-6 suggests that initial efforts at cost control may have become even less effective three to six months after the reforms. 


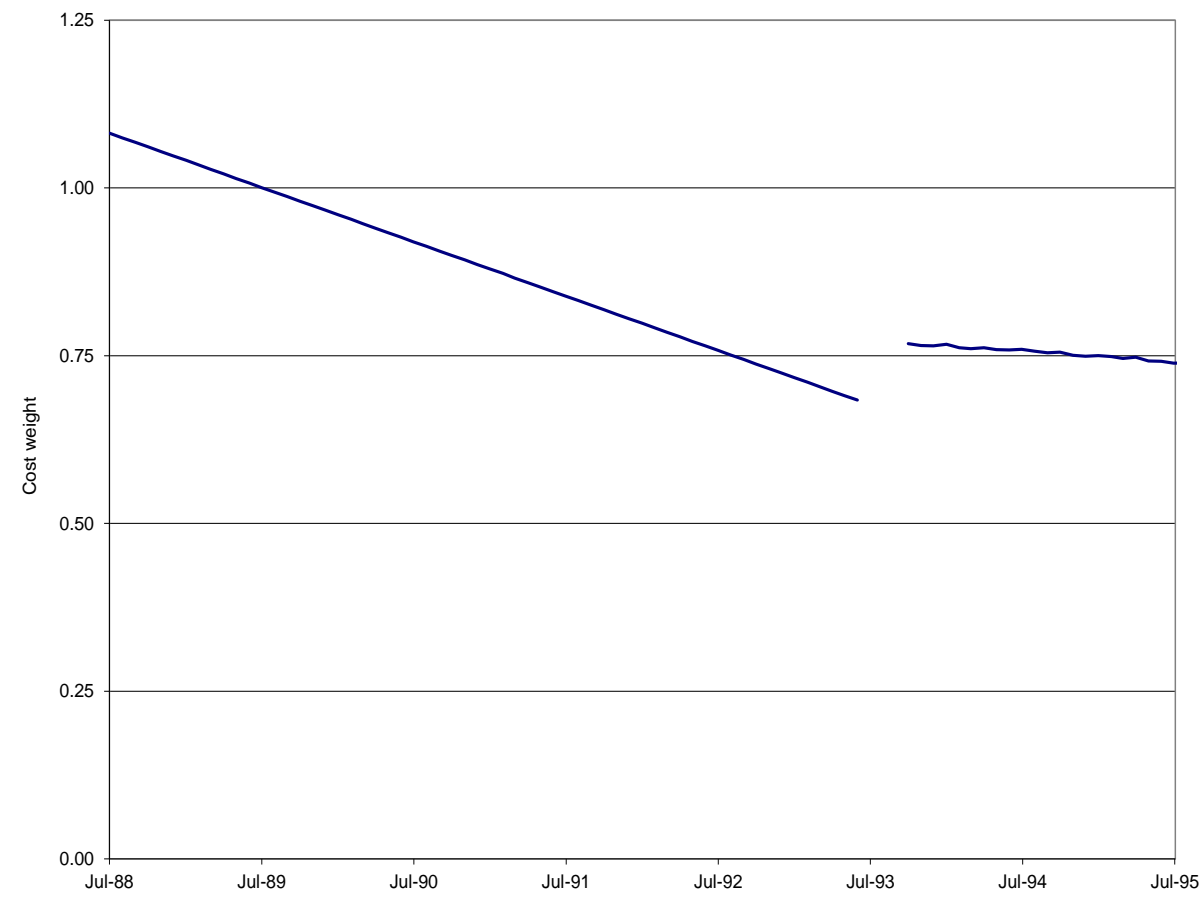

Figure A.5: Model of cost weight with a linear time trend (model 4-3), censored data

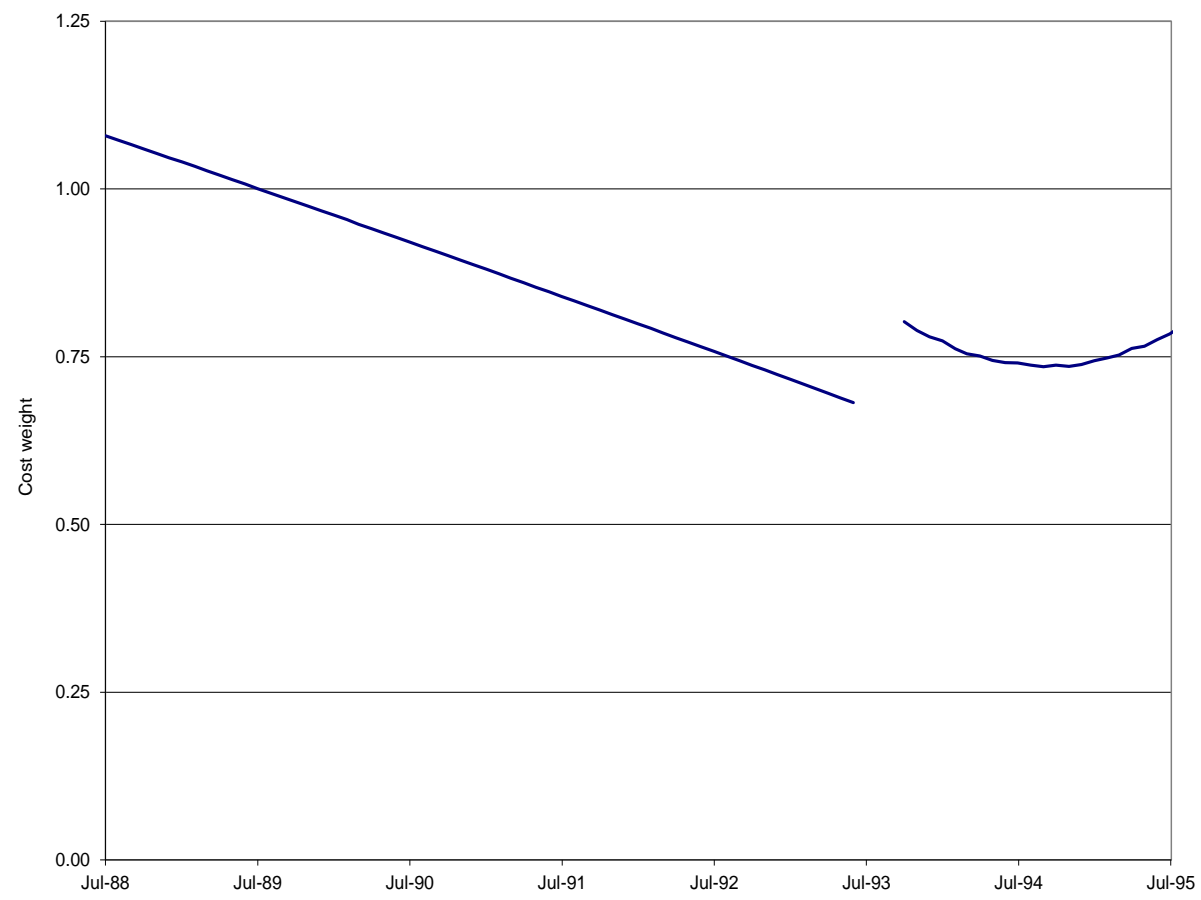

Figure A.6: Model of cost weight with a quadratic time trend (model 5-3), censored data 


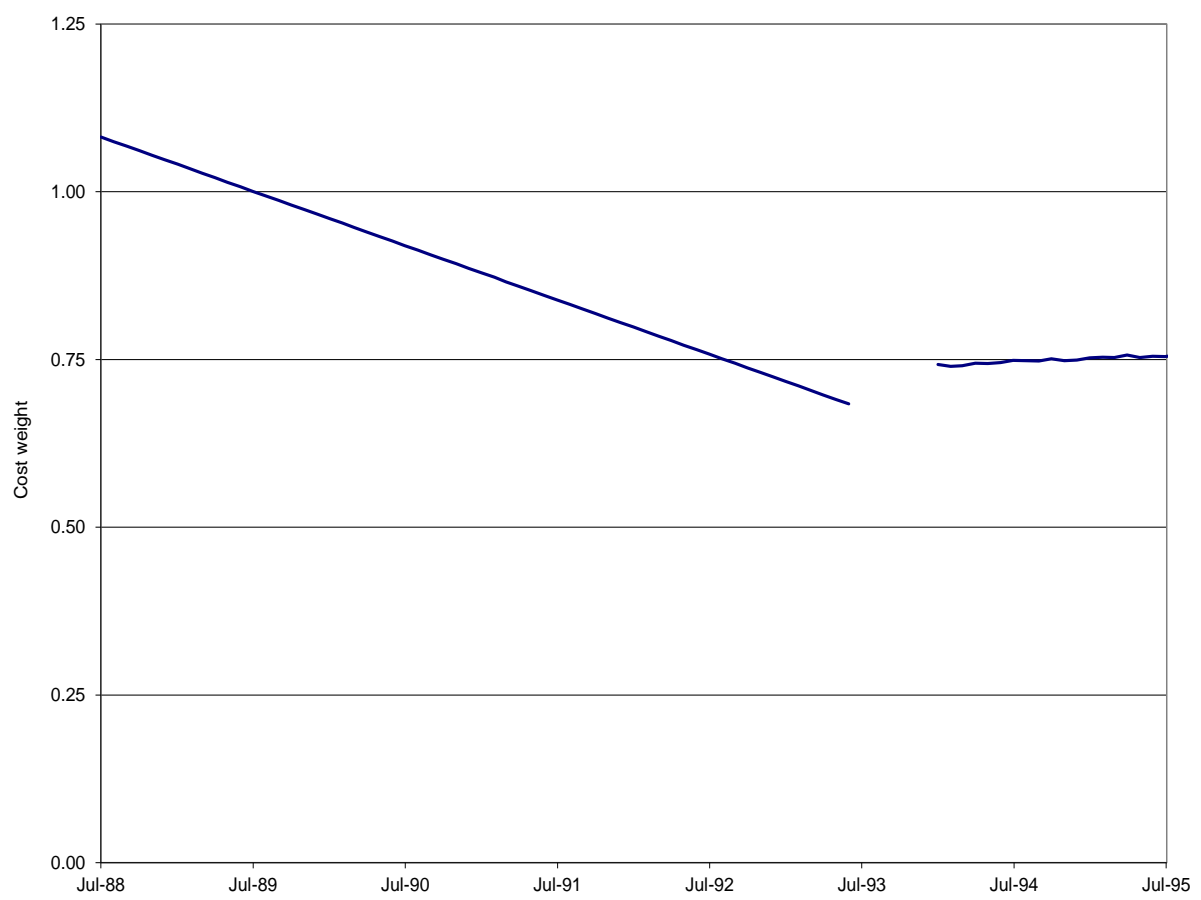

Figure A.7: Model of cost weight with a linear time trend (model 4-6), censored data

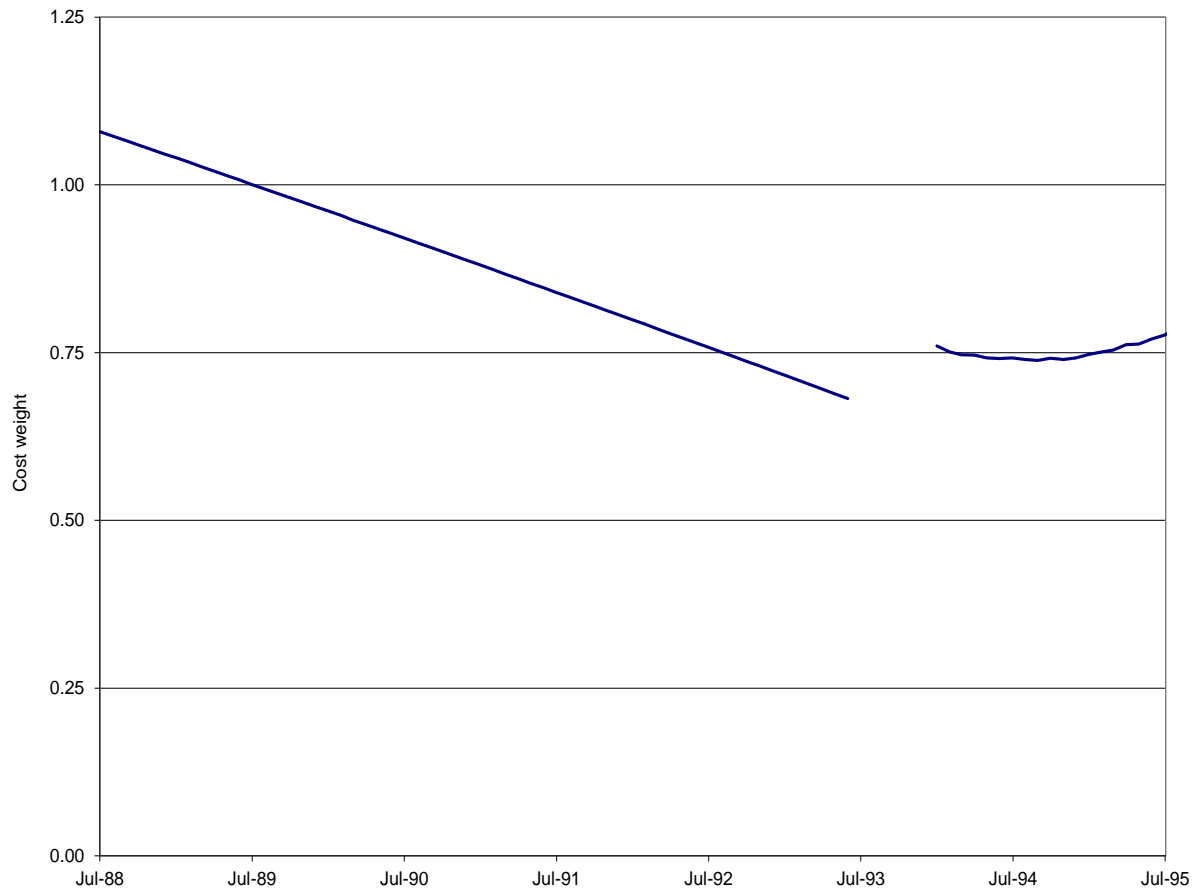

Figure A.8: Model of cost weight with a quadratic time trend (model 5-6), censored data 


\section{A.2 Appendices to Chapter 3}

\section{A.2.1 Delegation model}

This appendix provides a motivation for the SBC-alternative model developed in Chapter 3. We outline a a simple delegation model, and show why a health funder might choose a delegated governance structure like a CHE over direct control. It also notes a risk of delegating to an agent with incongruent incentives via an incomplete contract.

We show that delegation becomes less attractive as the interests of the funder and the administrator diverge, which is a conventional result from the literature. This result suggests why a funder might consider reallocating decision-making to another agent whose incentives can be more easily aligned with the funder's interests. This model also suggests that a funder might offer an administrator strong incentives for specific targets in order to align the administrator's incentives with the funder's interests.

In the case of AHB hospitals the funder delegated authority to a set of intermediate agents, a hospital management team. The CHE reformers believed the AHB hospital management team was too accommodating of clinical staff suggestions as the management layer lacked strong enough incentives from the funder. CHEs saw the triumvirate management team replaced by a single administrator, a CEO, who was given strong incentives for cost control. The reformers expected that this structure and set of incentives would better align the delegated agent's interests with those of the funder, thereby improving costefficiency from the funder's perspective.

\section{Background}

Delegation may benefit a funder despite incomplete contracts and imperfectly aligned incentives $5^{5}$ This conclusion is consistent with a funding system that delegates rationing decisions to an administrator, such as in CHEs, as a way to lower total costs.

\footnotetext{
${ }^{5}$ Our modelling approach is similar to Aghion \& Tirole's (1997) model of an organisational hierarchy. An organisation may have several tiers down which authority may be delegated.
} 
In the model below, delegation shifts treatment decisions to a better informed actor, an administrator. The model identifies why a funder might choose to delegate authority. Delegation takes advantage of an administrator who is better informed than the funder about cost-related conditions. It allows the administrator to ration treatment according to specific conditions rather than using a generic rule, which an uninformed funder would use. Delegation introduces tension where the funder and administrator's interests differ, as the administrator may use her authority to pursue her own interests rather than the funder's objective.

\section{Play of the game}

The model outlines the potential advantages of delegating the authority to decide whether a patient should receive some costly treatment. ${ }^{6}$ The model has two players: a funder $(F)$ and an administrator $(A) \cdot 7$ A patient's condition affects her cost of treatment, $c$, and these costs have a publicly known distribution $c \sim G]^{8}$ The administrator observes a patient's condition and knows the corresponding cost of treatment.9 We assume that the funder has all the bargaining power ex ante, but does not observe the patient's condition. We also assume that the funder is unable to verify the administrator's information, so a given patient's condition is non-contractible information 10 This means that the funder cannot enforce contractual provisions that depend on the patient's condition. For example, the

\footnotetext{
${ }^{6}$ This model assumes that a patient accepts treatment if it is offered. This assumption is revisited in Chapter 5 .

${ }^{7}$ The model assumes the agent potentially has two roles: an administrator, who makes rationing decisions according to the authority delegated to her and a specialist, who has private information about a patient's condition. This assumption may be interpreted as a situation where an administrator's and a specialist's incentives are closely aligned so they effectively make a single, joint, choice. Chapter 4 divides these roles between two actors who are involved in running the organisation. This division explicitly allows for the interests of the administrator and the specialist to diverge.

${ }^{8} \mathrm{~A}$ more general interpretation is possible, where the patient's condition could represent a wide range of cost-related factors about which the funder is uncertain ex ante.

${ }^{9}$ Although the funder concentrates on one type of public expenditure, for example rationing health services, we assume that the funder is equivalent to a benevolent social planner who acts in the best interests of society. Here, $c$ may be interpreted as the marginal social cost of a particular treatment, so a health funder and a social planner place the same value on the resources.

${ }^{10}$ Non-contractibility refers to a situation where a principal cannot observe or verify some relevant variable(s).
} 
funder cannot enforce a rule that requires the administrator to treat a patient only if that patient's condition exceeds the funder's cost-benefit threshold.

The steps of the game are as follows:

1. Nature $N$ chooses the patient's condition (and hence treatment cost) $c \sim G$.

2. $F$ chooses whether to delegate authority over the treatment decision to $A, D \in\left\{d_{0}, d_{1}\right\}$.

3. If $F$ retains control $\left(D=d_{0}\right)$ then he chooses whether to offer treatment or not, $T_{F} \in\{T, N\}$. If he delegates authority $\left(D=d_{1}\right)$ to $A$ then...

4. $A$ observes $c$ and she chooses whether to treat or not, $T_{A} \in\{T, N\}$.

The funder's objectives may differ from those of the administrator. To capture this incongruence we assume that both the funder and administrator derive some benefit from treating patients, but that they may value this benefit differently. In particular, we assume that an administrator may prefer to treat more often than the funder 11

An administrator may prefer more treatment for a number of reasons. She may receive a private benefit from treating a patient. This assumption is plausible where the administrator is a medical specialist, or concurs with a specialist, and specialists acquire valuable human capital through learning by doing. Alternatively an administrator may prefer to agree to extra treatment rather than to control costs in order to avoid pressure from demanding or concerned patients. In contrast, a funder who is remote from this interaction might be more concerned with cost. Alternatively, an administrator and a funder may have different perspectives. The administrator may focus on a particular service, sector or location in which she operates. The funder may have a broader perspective that reflects his responsibility to allocate resources across a range of services or sectors.

The extra benefit the administrator receives from treating a patient is represented by a parameter $0 \leq \beta$. If $0<\beta$, the administrator has a lower treatment threshold than the

\footnotetext{
${ }^{11}$ In a model with treatment intensity or quality this assumption might be interpreted as an administrator preferring safer, more complex or costly treatment rather than greater quantity.
} 
funder for a specific health condition, given its corresponding cost 12

The players' payoffs are noted below. The players would prefer to treat a patient if the perceived benefit exceeds the cost of treatment. Both the funder and administrator believe treatment may be beneficial, that is $0 \leq v$. The parameter $v$ is a minimum treatment threshold that is common to both the funder and administrator, such as a health service target. We assume it equals the social marginal benefit of treatment ${ }^{13}$ Differences in the value that the funder and the administrator place on treatment are represented by $\beta$ :

$$
\begin{aligned}
& U_{F}= \begin{cases}v-c & \text { with treatment } \\
0 & \text { without treatment }\end{cases} \\
& U_{A}= \begin{cases}v+\beta-c & \text { with treatment } \\
0 & \text { without treatment. }\end{cases}
\end{aligned}
$$

Figure A.9 summarises the steps of the game and the players' payoffs.

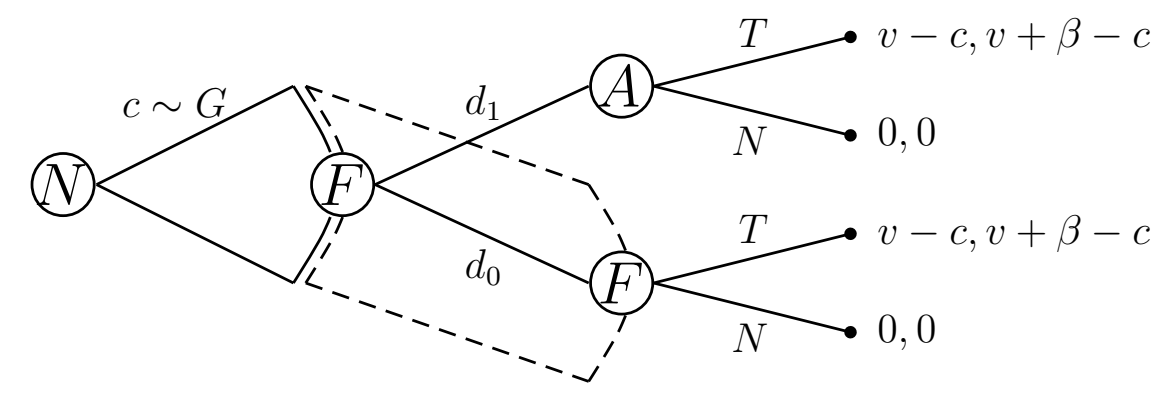

Figure A.9: Game tree for simple delegation model

\section{Solving the game}

The funder's delegation choice depends on how well the administrator's treatment choices would align with his preferences. Whenever the funder would offer treatment, the ad-

\footnotetext{
${ }^{12}$ This section focuses on the case where an administrator prefers to treat more than a funder, i.e. $0<\beta$.

${ }^{13}$ It is possible that a health funder's assessment of a health service's value differs from those of politicians or a social planner responsible for a broader set of prioritisation decisions.
} 
ministrator's positive treatment bias means the administrator would also offer treatment. When $v<c<v+\beta$ the administrator prefers treatment but the funder would not. The funder's uncertainty about $c$ means he may withhold treatment that, if informed, he would offer, i.e. those for whom $c<v \leq E[c]$. Informed, and if authorised, the administrator would offer such treatment.

The funder faces a potential trade-off in the delegation choice. Delegation allows the better informed actor to make the treatment choice. When the funder's and administrator's preferences perfectly align, delegation is desirable from the funder's perspective. When the administrator's preferences are incongruent with the funder's preferences, the administrator would offer treatment to some patients to whom the funder would prefer to withhold it, i.e. those for whom $v<c<v+\beta$. The delegation choice requires the funder to balance the cost of the administrator's inappropriately generous choices with that of the funder's poorly informed ones. The dilemma is characterised formally below.

Equation A.2 shows the funder's expected utility without delegation:

$$
\begin{aligned}
U_{F} & =\int_{0}^{1}(v-c) d G \\
& =P(0<c<1)(v-E[c]) \\
& =v-E[c] .
\end{aligned}
$$

Based on equation A.2, the funder's treatment choices at step 3 are as follows:

$$
T_{F}=\left\{\begin{array}{lll}
T & \text { if } E[c] \leq v & \text { yielding } E\left[U_{F}\right]>0 \\
N & \text { if } v<E[c] & \text { yielding } E\left[U_{F}\right]=0
\end{array}\right.
$$

The funder would offer treatment to all patients if the average patient's social benefit exceeds the social cost. Ex ante as the funder does not know the cost of treating a particular patient $c$, the funder may approve some inappropriate treatment, i.e. where $E(c) \leq v<c$. If $v<E[c]$, the funder declines all treatment and gets a payoff of 0 . This choice inappropriately denies some patients treatment, i.e. those with $c<v$. 
Delegation can improve the funder's payoff. With delegation, the administrator can accurately identify a patient's treatment cost, and only treats those patients where the cost does not exceed the administrator's treatment threshold, i.e. $c<v+\beta$. This yields:

$$
\begin{aligned}
U_{F} & =\int_{0}^{v+\beta}(v-c) d G+\int_{v+\beta}^{1}(0) d G \\
& =P(c<v+\beta)(v-E[c \mid c<v+\beta]) .
\end{aligned}
$$

The desirability of delegation depends on whether the expected cost of treatment, $E[c]$, is greater than the benefit of treatment, $v$. In the first case, where $E[c] \leq v$, delegation improves the funder's payoff. In the second case, where $v<E[c]$, the funder has a substantive choice: delegation may increase or decrease the funder's payoff.

In the first case, the funder would treat all patients where the average patient's cost is less than the funder's evaluation of the benefit, $E[c] \leq v$. Delegation can lower the upper treatment threshold as the administrator rations treatment where $v+\beta \leq c$. In this case, delegation restrains the funder's inappropriate (over-)treatment decisions. Equation A.5 below shows the condition for delegation to increase the funder's expected payoff.

The administrator would deny treatment to patients that do not meet her threshold, $v+\beta<c$. For this group of patients, the expected cost of treatment is greater than the funder's assessment of the social benefit, $v<E[c \mid v+\beta<c]$.

In the first case, where $E[c] \leq v$, funder would treat these patients if she does not delegate treatment authority. This would incur higher costs and lower his expected payoff. Here, his net payoff is greater under delegation than under direct control:

$$
v-E[c]<P(c<v+\beta)(v-E[c \mid c<v+\beta])
$$

The left hand side of equation (A.5) is the funder's expected payoff from treating all patients without delegation, as in equation (A.2). The right hand side of equation A.5 is the funder's expected payoff under delegation, where the administrator denies treatment to those patients that do not meet her treatment threshold, i.e. $c<v+\beta$. The second line of equation A.5 is the cost that the funder would incur without delegation from 
treating patients whose cost exceed the administrator's threshold. Equation A.5 is true if the funder expects the administrator to deny treatment to at least one patient who does not meet the administrator's cost-benefit threshold, $P(c<v+\beta)<1.14$ Therefore, delegation raises the funder's payoff when $E[c]<v$.

Equation A.5 may be interpreted as follows. For a given level of benefit, the administrator can lower costs by denying treatment to those who do not meet her threshold, but who the funder would have treated. This lowers expected costs, $E[c \mid c<v+\beta]<E[c] \leq v$. Where the administrator withholds treatment it is in the funder's interests. The administrator's restraint reflects her ability to target treatment on the basis of a particular patient's characteristics, which the funder lacks the information to do. In this case, delegation increases the funder's expected payoff despite the incongruence.

In the second case, where $v<E[c]$, the funder faces a substantive delegation choice. Deviation between the funder's and the administrator's preferred treatment choice is not always in the funder's interests. The administrator has a lower treatment threshold than the funder, so the funder would not always approve treatment when the administrator would. If the funder retains control and $v<E[c]$, he chooses not to any treat patients, yielding $U_{F}=U_{A}=0$. Comparing this payoff with that under delegation, in equation A.4, delegation can improve the funder's expected payoff if the following inequality holds:

$$
\begin{aligned}
& 0<\int_{0}^{v+\beta}(v-c) d G \\
\Rightarrow & 0<P(c<v+\beta)(v-E[c \mid c<v+\beta]) \\
\Rightarrow & E[c \mid c<v+\beta]<v .
\end{aligned}
$$

Delegation results in both appropriate $(c<v)$ and inappropriate $(v<c<v+\beta)$ treatment decisions from the funder's perspective. Equation A.6 implies that delegation is

\footnotetext{
${ }^{14}$ The administrator's cost-benefit threshold is higher than the funder's threshold, as $0<\beta$. Under this assumption, the administrator would not deny treatment to anyone to whom the funder would offer it, were the funder fully informed.
} 
desirable if the funder expects the net benefit of treating both appropriate and inappropriate patients to outweigh the cost of under-treatment resulting from his choice. Although the funder acknowledges the administrator may offer treatment to inappropriate patients, delegation remains preferable where he expects the typical patient to whom the administrator offers treatment to be worthy. The larger the incongruence between the funder and the administrator, that is a larger $\beta$, the less likely delegation would benefit the funder.

The funder's joint delegation-treatment choice $A T_{F}$ is as follows, where $d_{1}$ represents delegation where the administrator rations treatment and $d_{0}$ represents no delegation and the funder directly controls a treatment decision:

$$
A T_{F}= \begin{cases}d_{1} & \text { if } E(c \mid c<v+\beta)<v \\ d_{0} & \text { if } v<E(c \mid c<v+\beta) .\end{cases}
$$

As an example, with a uniform distribution on $[0,1], E(c \mid c<v+\beta)=\frac{v+\beta}{2}$. Hence equation A.6 is equivalent to the following inequality:

$$
\begin{gathered}
\frac{v+\beta}{2}<v \\
\Rightarrow \beta<v .
\end{gathered}
$$

Equation A.8 suggests delegation is preferable where the misalignment between the funder and the administrator, $\beta$, does not exceed a threshold, $v 15$

${ }^{15}$ Assuming $c \sim U(0,1)$ the expression in equation A.9 is equivalent to:

$$
0<v\left(v-\frac{v}{2}\right)+\beta\left(v-\frac{2 v+\beta}{2}\right) \Rightarrow \beta<v .
$$

Delegation is preferable if the expected cost of the administrator's over-treatment is less than the expected benefit of appropriately treating patients that the funder would otherwise failed to treat. 
The intuition for the threshold is clear from rearranging equation A.4 and relating it to the funder's zero payoff under direct control:

$$
\begin{aligned}
& 0<\int_{0}^{v}(v-c) d G+\int_{v}^{v+\beta}(v-c) d G+\int_{v+\beta}^{1}(0) d G \\
& 0<P(c<v)(v-E[c \mid c<v))+P(v<c<v+\beta)(v-E[c \mid v<c<v+\beta]) .
\end{aligned}
$$

Note that when $c<v$ then $P(c<v)(v-c)$ is positive. That is, delegation results in appropriate treatment of some patients that the funder would have denied under the direct control regime. When $v<c<v+\beta$, however, then $P(v<c<v+\beta)(v-c)$ is negative. This represents the cost of over-generous treatment. That is, delegation results in the treatment of some patients that the funder would prefer to deny it to.

Figure A.10 illustrates the funder's payoffs when he delegates authority, assuming $c \sim$ $U(0,1)$. The dark line shows the funder's payoff for a particular cost of treatment.

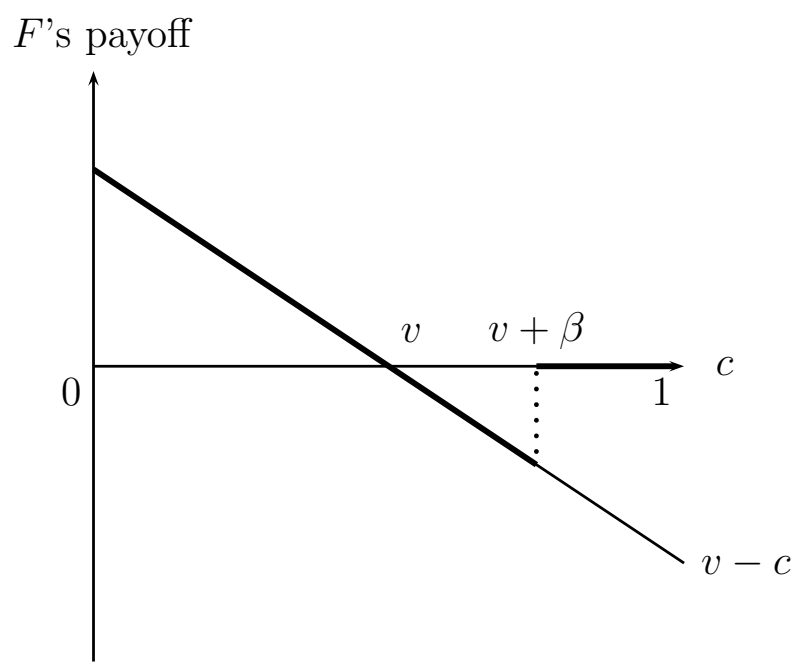

Figure A.10: Example of F's payoffs under delegation

The delegation choice depends on the size of the funder's expected disutility from overgenerous treatment relative to the utility from allowing appropriate treatment. If $c \sim$ $U(0,1)$, the funder's expected cost from delegation is the triangle between $v$ and $v+\beta$, and the expected benefit is the triangle to the left of $v$. Delegation can be beneficial for the funder even if the funder's and administrator's incentives are imperfectly aligned. 


\section{A.2.2 Soft budget constraint model}

This appendix provides a motivation for why health reformers might expect performancebased bonuses for CEOs to help rein in costs. This appendix outlines a conventional SBC model based on our stylised CHE organisational hierarchy. The model in Chapter 3 extends this conventional model to incorporate three important features of the CHE reforms that conventional SBC models neglect.

The previous appendix outlined conditions under which a funder might delegate decisionmaking authority to an administrator. This appendix examines what budget and bonus a funder might set for an administrator to whom authority has been delegated. It investigates whether performance bonuses can undermine an administrator's incentives to pursue cost efficiency under a particular set of circumstances.

This appendix outlines two models. The first model introduces the concept of an SBC, where the funder is willing to revise the organisation's budget once some uncertainty is resolved. In this model, a budget increase, that is a relaxation of an initial budget constraint, represents a failure to achieve efficiency.

The second model extends the first. It shows how an appropriate set of budgets and bonuses can be used to achieve an ex post efficient outcome. The model does not result in an SBC-type result, in the sense that a funder would not benefit from being able to commit to the original budget. This model may explain the first criticism about bonus payments despite failures to achieve budget targets. However, this simple model only partially addresses the first criticism addressed by the thesis. It does not fully explain the incentive role of both budget revision and bonus payments in an environment where a poorly informed funder delegates authority to a better informed agent. A fuller explanation is the territory of the chapter's main model in section 3.4 .

Both models in this appendix assume that contracts are incomplete and the funder cannot credibly commit to an HBC. 


\section{SBC model with a single effort stage}

Below we set up an SBC model that illustrates some conventional SBC results.

\section{Play of the game}

This model has two players: a funder $(F)$ and an administrator $(A)$. It is a one-stage model where the administrator has one opportunity to provide effort that affects the organisation's costs. However, the funder has two opportunities to influence the budget and bonus. In the first stage the funder offers an initial budget before learning the cost conditions that the organisation faces. In the second stage, the funder can offer a revised contract, in light of the administrator's choice of effort and the organisation's costs, that alters the budget and bonus.

The steps of the game are as follows:

1. F makes an offer of a budget and bonus, $\left\{b_{1}, w_{1}\right\} \in \mathbb{R}_{+}^{2}$.

2. A observes the offer and decides whether to accept it. If not, then both players get zero. Otherwise ...

3. $A$ chooses an effort level $e \in\{0,1\}$, which determines costs $c(e) 16$

4. $F$ observes $e$. If $F$ wishes, he can revise his offer, but only by making it more generous; i.e. he chooses $\left\{b_{2}, w_{2}\right\} \in \mathbb{R}_{+} \backslash\left[0, b_{1}\right) \times \mathbb{R}_{+} \backslash\left[0, w_{1}\right)$.

5. A observes the revised offer and decides whether to accept it, or reject it in favour of the original contract.

The funder gets a benefit of $v$ if the budget is balanced, i.e. if $c(e) \leq b$, and otherwise wants to keep total costs, $b+w$, as low as possible. He gets $v-c(e)-w$ if the budget is balanced, and $-c(e)$ otherwise. The administrator gets $w-e$ if the budget is balanced, and $-e$ otherwise. We assume that even with low effort the funder wishes the hospital

\footnotetext{
${ }^{16}$ This section assumes effort is discrete, taking a value of 0 or 1 ; the qualitative results are the same if effort is continuous, $e_{1} \in \mathbb{R}_{+}$.
} 
to operate, $c(0) \leq v$. This assumption relates to the idea of an $\mathrm{SBC}$, where the on-going operation of an organisation is valuable and may be worthwhile even in the face of a deficit. In the context of New Zealand's extensive public secondary health service, the widespread closure of public hospitals that ran deficits, and consequent service shortage, would likely have been less politically palatable than deficits.

Figure A.11 illustrates the steps of the game and the players' payoffs.

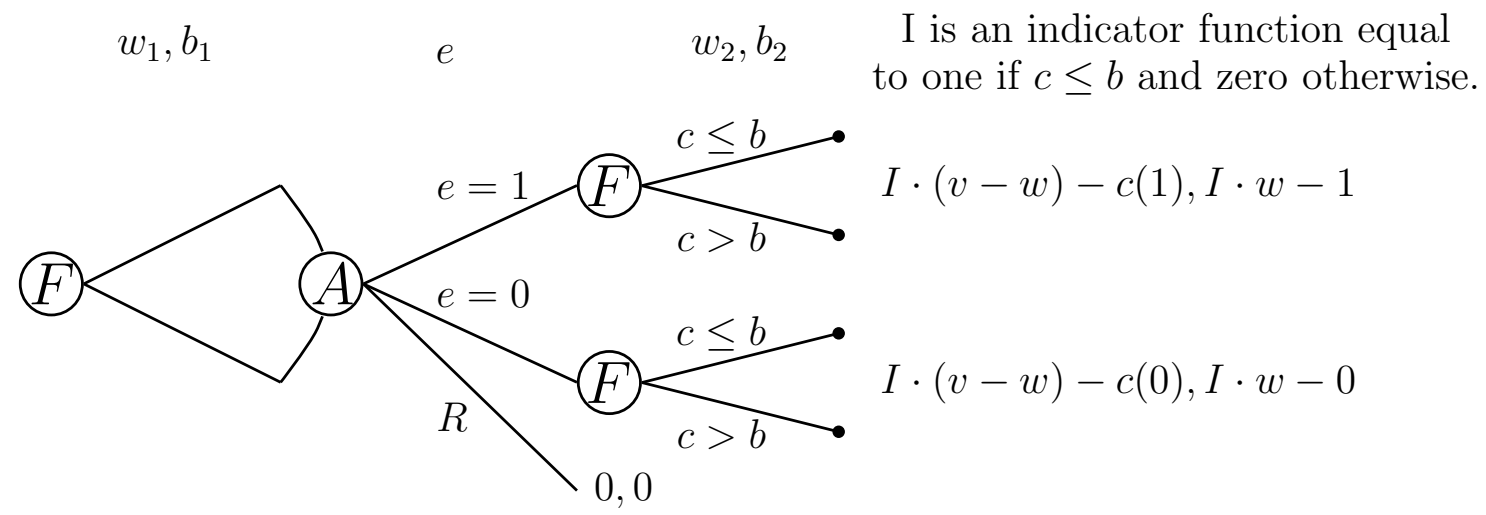

Figure A.11: Game tree for first SBC model

\section{Outcomes of the game}

In step 4, the funder will ensure that the budget is high enough to cover the costs determined in step 3. That is, $b_{2}=\max \left\{b_{1}, c(e)\right\}$. The funder wishes to minimise costs subject to meeting the budget constraint $c(e) \leq b$. In the first step, he would like to offer the administrator a contract that keeps the administrator on her participation constraint. The best outcome for the funder could be characterised as follows:

$$
e=1, w_{1}=1, b_{1}=b_{2}=c(1)
$$

While this is the efficient outcome, it is not an equilibrium outcome. Anticipating the funder's reaction in step 3 , the administrator could do better under such a contract by putting in low effort $e=0$. If $b_{1}<c(0)$, the funder would prefer to increase the budget in stage 2 to $b_{2}=c(0)$, rather than violate the budget constraint, as $c(0)<v$. The administrator would receive a bonus $w_{1}$ even if she provided low effort. Therefore in step 
3 the administrator will choose $e=0$, anticipating a payoff of $w$ rather than $e=1$ and a payoff of $w-1$.

The funder's inability to commit to an HBC means that the administrator prefers inefficiently low effort in step 3. The funder is unable to overcome this perverse incentive using a bonus because the administrator's actions are non-contractible ex ante and the funder cannot credibly commit to a low initial budget. In equilibrium, the funder cannot encourage the administrator to provide early effort. As the bonus serves no useful role in promoting effort, the funder can hold the administrator to her participation constraint with a bonus of $w=0$. The administrator is indifferent and accepts, yielding the funder a non-negative payoff. The budget set in the first stage is irrelevant to the play of the game as long as it is less than $c(0)$. The ultimate budget, however, is inefficiently high as it does not minimise overall costs: $b_{1}<b_{2}=c(0)$. The choices in the equilibrium outcome are as follows:

$$
e=0, w_{1}=0, b_{1}=b_{2}=c(0)
$$

Although the funder has all the ex ante bargaining power, it does not allow him to design a contract that results in an efficient outcome because of his inability to commit to an HBC ex post (as we assume the funder prefers to preserve the organisation rather than liquidate it). There is an SBC as the funder would be willing to revise the budget ex post, if necessary, to cover costs and this leads to ex ante inefficiency. The funder is willing to set the initial budget above the cost-minimising level, $c(1)$, as he does not expect the administrator to provide effort, $e=1$, in equilibrium. This result differs from the standard result of ex post budget increases of SBC models ${ }^{17}$ The next section examines how bonuses might be used to encourage optimal effort.

\footnotetext{
${ }^{17}$ This difference stems from the funder's willingness in this model that the organisation operates despite a high-cost budget. Conventional SBC models tend to concentrate on the decision to set up a new organisation and where the funder would not invest in a high-cost organisation if he knew it would be high cost ex ante. This model focuses on an organisation that has already been established and the merits of its on-going operation, for example, whether to shut down an existing, high-cost public hospital.
} 


\section{SBC model with two effort stages}

This model builds on the one-stage SBC model above. We now assume that the administrator has two opportunities to influence costs. Costs depend on her effort choices in both stages, i.e. $c=c\left(e_{1}, e_{2}\right)$. Effort remains non-contractible, but the funder observes $c\left(e_{1}, \cdot\right)$. The budget constraint is contractible ex ante, as costs are observable. That is, the funder can make the administrator's bonus contingent on costs relative to the budget.

\section{Play of the game}

This game adds a second opportunity, or stage, for the administrator to provide effort. The steps of the game are as follows:

1. $F$ offers a budget and a bonus, $\left\{b_{1}, w_{1}\right\} \in \mathbb{R}_{+}^{2}$.

2. A observes the offer and decides whether to accept it. If not, then both players get zero. Otherwise ...

3. $A$ chooses an effort level $e_{1} \in\{0,1\}$.

4. $F$ observes $e_{1}$. $F$ can make his offer more generous; i.e. he chooses $\left\{b_{2}, w_{2}\right\} \in$ $\mathbb{R}_{+} \backslash\left[0, b_{1}\right) \times\left[0, w_{1}\right){ }^{18}$

5. $A$ chooses another effort level, $e_{2} \in\{0,1\}$.

This model assumes that effort lowers costs but a second unit of effort has less impact on costs than the first. Early effort is assumed to be more effective than later effort in restraining costs, and second stage effort has no additional impact on $c$ if the administrator provided effort in stage one, $e_{1}=1$. These assumptions are summarised as: $c(1,1)=$ $c(1,0)<c(0,1)<c(0,0)$. In addition, the model assumes the sum of operational costs plus the cost of the administrator's effort is lower than costs without effort, $c(0,1)+1<c(0,0)$. This implies that it can be worthwhile for the funder to pay for at least one unit of effort.

\footnotetext{
${ }^{18}$ We assume that $F$ cannot replace the initial contract $A$ accepted in step 2 without $A$ 's agreement. Therefore a revised offer must be more generous for $A$ to be willing to replace the initial contract.
} 
The players' payoffs in the two-stage game are as follows:

$$
\begin{aligned}
& U_{F}= \begin{cases}v-c\left(e_{1}, e_{2}\right)-w & \text { if } c\left(e_{1}, e_{2}\right) \leq b_{2} \\
-c\left(e_{1}, e_{2}\right) & \text { otherwise }\end{cases} \\
& U_{A}= \begin{cases}w-\left(e_{1}+e_{2}\right) & \text { if } c\left(e_{1}, e_{2}\right) \leq b_{2} \\
-\left(e_{1}+e_{2}\right) & \text { otherwise. }\end{cases}
\end{aligned}
$$

\section{Outcomes of the game}

As in section A.2.2, we assume that the funder wishes the hospital to operate even with the minimum level of effort, i.e. $c(0,0)<v$. The funder prefers the administrator to provide effort in the first stage as it is more effective than later effort at controlling operation costs. As the funder wishes to minimise total costs subject to meeting the budget constraint, $c\left(e_{1}, e_{2}\right) \leq b$, he would like to offer the administrator a contract that keeps her on her participation constraint, that is a contract which pays the administrator the minimum necessary for the administrator to accept the contract and to provide cost-minimising effort. This outcome involves the following choices:

$$
e_{1}=\arg \min _{e} e+c(e), e_{2}=0, b=c\left(e_{1}\right), w=e_{1}
$$

The efficient outcome, where the administrator provides early effort, is equivalent to section A.2.2 but now the efficient outcome is also the equilibrium outcome. In section A.2.2, an administrator might benefit from shirking by providing zero effort in step 3 , The second stage gives the funder leverage over an administrator's choice of effort because the administrator moves last. Now, the administrator cannot rely on the funder to relax the budget constraint to ensure the budget covers costs, even when the administrator provides the minimum level of effort. A funder can use the bonus to compensate an administrator for her effort and can now credibly commit to the initial budget as it is the administrator who has the last chance to balance the budget, rather than the funder.

If the funder finds that $e_{1}=0$ in step 4 , in the following sub-game he can set $b_{2}=c(0,1)$ and $w_{2}=1$. This gives the administrator an incentive to provide effort in the second 
stage, but the cost-minimising scheme leaves her with a net payoff of zero. In step 1, the funder can contract for early effort by offering $w_{1}=1$ and $b_{1}=c(1,0)$. This contract also gives the administrator a zero payoff, so she is indifferent to when she provides effort. In this game as the funder can write a contract so that in either stage the administrator is willing to provide effort and receives a zero payoff. In equilibrium, the funder can achieve his desired outcome of early effort in a way that minimises costs.

This model shows that bonuses may be used to overcome the undesirable incentive effects of incomplete contracts that can result in a standard SBC-type result of inefficiently large budgets. The result of this model suggests that the standard SBC result of inefficient budget revision is not necessarily a universal outcome of non-contractible effort. Even with non-contractible effort, the administrator can be held to her reservation level of utility and the budget is both ex ante and ex post optimal for the funder.

This model suggests that bonuses may have desirable incentive effects where effort is non-contractible. But it does not predict budget increases in combination with bonus payments to the delegated agent. Therefore the model in this section partially addresses the criticism that CHEs paid performance bonuses despite budget increases. Section 3.4 of Chapter 3 adapts the two-stage effort model to address this issue more fully. 


\section{A.3 Appendices to Chapter 4}

\section{A.3.1 Proof of the uniqueness of an NPE outcome}

In order to examine how a change in parameter values alters the equilibrium outcome in Chapter 4, we determine whether a particular set of parameters corresponds to a particular outcomes.

This appendix: shows that a NPE is unique if it exists; and, a NPE might exist for the entire range of $\theta$; and, outlines the conditions under which an outcome is guaranteed to exist. It provides a proof of propositions (1) and (2) and provides a supplementary proposition, Proposition (3), which focuses on the conditions for the existence and uniqueness of a NPE for $\theta \in(\underline{\theta}, \bar{\theta})$.

Recall the following terminology. The first set divides up the support of $H$ with reference to the specialist's preferences ${ }^{19}$

$$
\begin{aligned}
& Z_{1}=\left\{\pi \mid 0 \leq \pi \leq \gamma_{12}\right\} \\
& Z_{12}=\left\{\pi \mid \gamma_{12} \leq \pi \leq \gamma_{13}\right\} \\
& Z_{23}=\left\{\pi \mid \gamma_{13} \leq \pi \leq \gamma_{23}\right\} \\
& Z_{2}=Z_{12} \cup Z_{23} \\
& Z_{3}=\left\{\pi \mid \gamma_{23} \leq \pi \leq 1\right\}
\end{aligned}
$$

The second set shows the thresholds at which the administrator is indifferent about funding a given treatment, $t_{i}$.

$$
\begin{aligned}
& \theta_{1} \text { solves }\left(1+\theta_{1}\right)=\frac{E\left[\pi \mid Z_{2}\right]}{\gamma_{12}} \\
& \theta_{2} \text { solves }\left(1+\theta_{2}\right)=\frac{E\left[\pi \mid Z_{2} \cup Z_{3}\right]}{\gamma_{12}} \\
& \theta_{3} \text { solves }\left(1+\theta_{3}\right)=\frac{E\left[\pi \mid Z_{23} \cup Z_{3}\right]}{\gamma_{23}} \\
& \theta_{4} \text { solves }\left(1+\theta_{4}\right)=\frac{E\left[\pi \mid Z_{3}\right]}{\gamma_{23}}
\end{aligned}
$$

\footnotetext{
${ }^{19}$ The $\gamma_{i}$ thresholds are defined in equation array 4.4 .3 on page 90
} 
Finally, recall that $\underline{\theta}=\min \left\{\theta_{1}, \theta_{4}\right\}$ and $\bar{\theta}=\max \left\{\theta_{2}, \theta_{3}\right\}$. As $\theta_{1}<\theta_{2}$ and $\theta_{3}<\theta_{4}$, this implies that $\underline{\theta}<\bar{\theta}$.

\section{Proof for proposition (1).}

Proposition 1 For $\underline{\theta}$ and $\bar{\theta}$ as defined above

i) A neologism-proof equilibrium with menu of treatments $T_{0}=\left\{t_{1}, t_{2}, t_{3}\right\}$ exists if and only if $\theta \leq \underline{\theta}$.

ii) A neologism-proof equilibrium with menu of treatments $T_{0}=\left\{t_{1}\right\}$ exists if and only if $\bar{\theta} \leq \theta$.

The first part of proposition (1) states that a neologism-proof equilibrium with menu of treatments $T_{0}=\left\{t_{1}, t_{2}, t_{3}\right\}$ exists if and only if $\theta \leq \underline{\theta}$.

An equilibrium outcome with the menu $T_{0}=\left\{t_{1}, t_{2}, t_{3}\right\}$ requires that the first three conditions in Table 4.6 are all satisfied. The two substantive conditions may be expressed as follows 20

$$
\begin{aligned}
& (1+\theta) \gamma_{12} \leq E\left[\pi \mid \pi \in Z_{2}\right] \Leftrightarrow(1+\theta) \leq\left(1+\theta_{1}\right) \\
& (1+\theta) \gamma_{23} \leq E\left[\pi \mid \pi \in Z_{3}\right] \Leftrightarrow(1+\theta) \leq\left(1+\theta_{4}\right)
\end{aligned}
$$

The threshold $\underline{\theta}$ is defined so that these two conditions are satisfied if and only if $\theta \leq \underline{\theta}$.

The second part of proposition (1) states that a neologism-proof equilibrium with menu of treatments $T_{0}=\left\{t_{1}\right\}$ exists if and only if $\bar{\theta} \leq \theta$. The two substantive conditions from Table 4.6 may be expressed as follows ${ }^{21}$

$$
\begin{gathered}
E\left[\pi \mid \pi \in Z_{2} \cup Z_{3}\right] \leq(1+\theta) \gamma_{12} \Leftrightarrow\left(1+\theta_{2}\right) \leq(1+\theta) \\
E\left[\pi \mid \pi \in Z_{23} \cup Z_{3}\right] \leq(1+\theta) \gamma_{23} \Leftrightarrow\left(1+\theta_{3}\right) \leq(1+\theta)
\end{gathered}
$$

\footnotetext{
${ }^{20}$ The conditions in Table 4.6 marked with a dagger are not substantive, and we do not consider them further in this proof. For example, the first condition for $T_{0}=\left\{t_{1}, t_{2}, t_{3}\right\}$ to be consistent with a NPE outcome, in row one of Table 4.6 is $E\left[\pi \mid o(\pi)=t_{1}\right] \leq(1+\theta) \gamma_{12}$. For $T_{0}=\left\{t_{1}, t_{2}, t_{3}\right\}, o^{*}(\pi)=t_{1}$ when $Z^{S}=\left[0, \gamma_{12}\right]$ and under the condition that $E\left[\pi \mid \pi \in Z_{1}\right] \leq(1+\theta) \gamma_{12}$. This condition is always true.

${ }^{21}$ If the second necessary condition for this equilibrium outcome in Table 4.6 is satisfied, then the first condition is also satisfied.
} 
By definition, these two conditions are satisfied if and only if $\bar{\theta} \leq \theta$.

Thus, proposition (1) ensures that an equilibrium outcome exists for $\theta \leq \underline{\theta}$ and $\bar{\theta} \leq \theta$.

\section{Proof for proposition (2).22}

Proposition 2 For $\underline{\theta}$ and $\bar{\theta}$ as defined above,

i) A neologism-proof equilibrium with the menu of treatments $T_{0}=\left\{t_{1}, t_{2}\right\}$ exists if and only if $\theta_{4} \leq \theta \leq \theta_{2}$.

ii) A neologism-proof equilibrium with the menu of treatments $T_{0}=\left\{t_{1}, t_{3}\right\}$ exists if and only if $\theta_{1} \leq \theta \leq \theta_{3}$.

The first part of proposition (2) states that a neologism-proof equilibrium with the menu of treatments $T_{0}=\left\{t_{1}, t_{2}\right\}$ exists if and only if $\theta_{4}<\theta \leq \theta_{2}$. This proof is similar to the first proof. The two substantive conditions for this equilibrium may be expressed as follows 23

$$
\begin{array}{r}
(1+\theta) \gamma_{12} \leq E\left[\pi \mid \pi \in Z_{2} \cup Z_{3}\right] \Leftrightarrow(1+\theta) \leq\left(1+\theta_{2}\right) \\
E\left[\pi \mid \pi \in Z_{3}\right] \leq(1+\theta) \gamma_{23} \Leftrightarrow\left(1+\theta_{4}\right) \leq(1+\theta)
\end{array}
$$

Thus, this equilibrium exists if and only if $\theta_{4} \leq \theta \leq \theta_{2}$.

The second part of proposition (2) states that a neologism-proof equilibrium with the menu of treatments $T_{0}=\left\{t_{1}, t_{3}\right\}$ exists if and only if $\theta_{1} \leq \theta \leq \theta_{3}{ }^{24}$

$$
\begin{array}{r}
E\left[\pi \mid \pi \in Z_{2}\right] \leq(1+\theta) \gamma_{12} \Leftrightarrow\left(1+\theta_{1}\right) \leq(1+\theta) \\
(1+\theta) \gamma_{23} \leq E\left[\pi \mid \pi \in Z_{23} \cup Z_{3}\right] \Leftrightarrow(1+\theta) \leq\left(1+\theta_{3}\right)
\end{array}
$$

\footnotetext{
${ }^{22}$ Appendix A.3.2 explores the intuition behind the two cases in this proposition, and what bias would lead to which outcome when only one exists.

${ }^{23}$ If the second condition below is true, then $\frac{E\left[\pi \mid Z_{2} \cup Z_{3}\right]}{\gamma_{23}}<\theta_{4}$, which implies the upper bound of condition $\mathrm{C} 1$ in Table 4.6 is also satisfied. Therefore, we only consider the lower bound in this proof, which is the first condition below.

${ }^{24}$ We focus on the second necessary condition for this outcome in Table 4.6 as one of two substantive conditions. We begin by noting that the first necessary condition for this outcome in Table 4.6 is satisfied if the second necessary condition is true.

We also note this outcome can be disrupted if $(1+\theta) \gamma_{12}<E\left[\pi \mid \pi \in Z_{2}\right]<(1+\theta) \gamma_{23}$, as shown in table 4.4 on page 96 . Therefore, for this outcome to be neologism proof either $E\left[\pi \mid Z_{2}\right] \leq(1+\theta) \gamma_{12}$ or $(1+\theta) \gamma_{23} \leq E\left[\pi \mid Z_{2}\right]$. The latter is impossible, so the former must hold for the outcome to be neologism proof and this is simply the second necessary condition for this outcome as given in Table 4.6
} 
These conditions are true if and only if $\theta_{1} \leq \theta \leq \theta_{3}$.

The following proposition focuses on the interval $(\underline{\theta}, \bar{\theta})$, as the first proposition guarantees uniqueness and existence elsewhere.

Proposition 3 There are six possibilities for the existence of a NPE in the range $(\underline{\theta}, \bar{\theta})$. i) If $\theta_{3}<\underline{\theta}=\theta_{1}<\theta_{2}=\bar{\theta}<\theta_{4}$ then neither a NPE with $T_{0}=\left\{t_{1}, t_{2}\right\}$ nor a NPE with $T_{0}=\left\{t_{1}, t_{3}\right\}$ exists, and the interval $(\underline{\theta}, \bar{\theta})$ has no NPE.

ii) If $\theta_{3}<\underline{\theta}=\theta_{1}<\theta_{4}<\theta_{2}=\bar{\theta}$ then a NPE with $T_{0}=\left\{t_{1}, t_{2}\right\}$ may exist in the interval $\left[\theta_{4}, \bar{\theta}\right]$ if the interval is non-empty, but the interval $\left(\underline{\theta}, \theta_{4}\right)$ has no NPE and a NPE with $T_{0}=\left\{t_{1}, t_{3}\right\}$ does not exist.

iii) If $\underline{\theta}=\theta_{1}<\theta_{3}<\theta_{2}=\bar{\theta}<\theta_{4}$ then a NPE with $T_{0}=\left\{t_{1}, t_{3}\right\}$ may exist in the interval $\left[\underline{\theta}, \theta_{3}\right]$ if the interval is non-empty, but the interval $\left(\theta_{3}, \bar{\theta}\right)$ has no NPE and a NPE with $T_{0}=\left\{t_{1}, t_{2}\right\}$ does not exist.

iv) If $\underline{\theta}=\theta_{1}<\theta_{3}<\theta_{4}<\theta_{2}=\bar{\theta}$ then NPEs with both $T_{0}=\left\{t_{1}, t_{2}\right\}$ and $T_{0}=\left\{t_{1}, t_{3}\right\}$ may exist if the relevant intervals, $\left(\underline{\theta}, \theta_{3}\right)$ and $\left(\theta_{4}, \bar{\theta}\right)$, are non-empty, but the interval $\left(\theta_{3}, \theta_{4}\right)$ has no NPE.

v) If $\theta_{3}<\underline{\theta}=\theta_{4}<\theta_{1}<\theta_{2}=\bar{\theta}$ then a NPE with $T_{0}=\left\{t_{1}, t_{2}\right\}$ may exist in the interval $[\underline{\theta}, \bar{\theta}]$ if the interval is non-empty, but a NPE with $T_{0}=\left\{t_{1}, t_{3}\right\}$ does not exist.

vi) If $\underline{\theta}=\theta_{1}<\theta_{2}<\theta_{3}=\bar{\theta}<\theta_{4}$ then a NPE with $T_{0}=\left\{t_{1}, t_{3}\right\}$ may exist in the interval $[\underline{\theta}, \bar{\theta}]$ if the interval is non-empty, but a NPE with $T_{0}=\left\{t_{1}, t_{2}\right\}$ does not exist.

There are six possibilities for the shape of the equilibrium correspondence in the interval $(\underline{\theta}, \bar{\theta})$ that are consistent with the assumptions on the $\theta$ thresholds given on page 191 . The first four parts of this proposition show that one or neither of the equilibrium outcomes exists for the interval $(\underline{\theta}, \bar{\theta})$, but if an outcome does exist in this range then it is unique. The last two parts show that an equilibrium outcome exists for the entire interval of $(\underline{\theta}, \bar{\theta})$, but it is either a NPE with $T_{0}=\left\{t_{1}, t_{2}\right\}$ or a NPE with $T_{0}=\left\{t_{1}, t_{3}\right\}$.

Taking the last two parts of Proposition (3) together with Proposition (1) implies that an equilibrium outcome can exist for the entire range of $\theta$, and the outcome is unique. 


\section{A.3.2 Example of NPE outcomes}

This appendix illustrates which of the NPE outcomes determined in Chapter 4 occur for particular values of the model parameters. The example considers how it is possible to move from an outcome involving all treatments to one of several outcomes where only a limited set of treatments would be authorised. In one case, the move may result in an outcome with higher treatment and total costs.

The example differs from the main chapter by adopting a specific distribution for $\pi$ and differs from appendix A.3.1 by varying the $\gamma$ thresholds rather than focusing on $\theta$.

The example assumes that $\pi$ has a uniform distribution, $\pi \sim U(0,1)$. By adopting this distribution, we can characterise the substantive equilibrium outcome conditions in Table 4.6 more specifically. In the figure below, we do not show the trivial or non-substantive conditions, which are marked with daggers in Table 4.6. We also simplify the presentation by letting $\gamma_{13}$ equal a weighted average of $\gamma_{12}$ and $\gamma_{23}: \gamma_{13}=\alpha \gamma_{23}+(1-\alpha) \gamma_{12}$. Table A.5 (on page 197) reproduces Table 4.6 on the basis of these two simplifications.

Figure A.12 on page 195 graphically summarises the results in Table A.5. This figure shows the areas where particular values of $\gamma_{12}$ and $\gamma_{23}$ satisfy the equilibrium conditions for the outcomes listed in table A.5 The areas correspond to the outcome labels: $A=\left\{t_{1}, t_{2}, t_{3}\right\}, B=\left\{t_{1}, t_{3}\right\}, C=\left\{t_{1}, t_{2}\right\}$ and $D=\left\{t_{1}\right\}$. Solid lines indicate boundaries where all the necessary conditions for a given outcome are satisfied. Dashed lines indicate that a single necessary condition is satisfied for the given equilibrium, but the other necessary conditions are not. Thus, zones bounded by solid lines indicate the various sets of parameter values that are consistent with a given equilibrium. By changing the values of $\gamma_{12}$ and $\gamma_{23}$, we can see which outcome is consistent with equilibrium as this will shift the solid and dotted lines. This would alter the zones consistent with the various outcomes.

\footnotetext{
${ }^{25}$ No parameter values that fall below the $45^{\circ}$ line satisfy our assumption that $\gamma_{12}<\gamma_{23}$.
} 


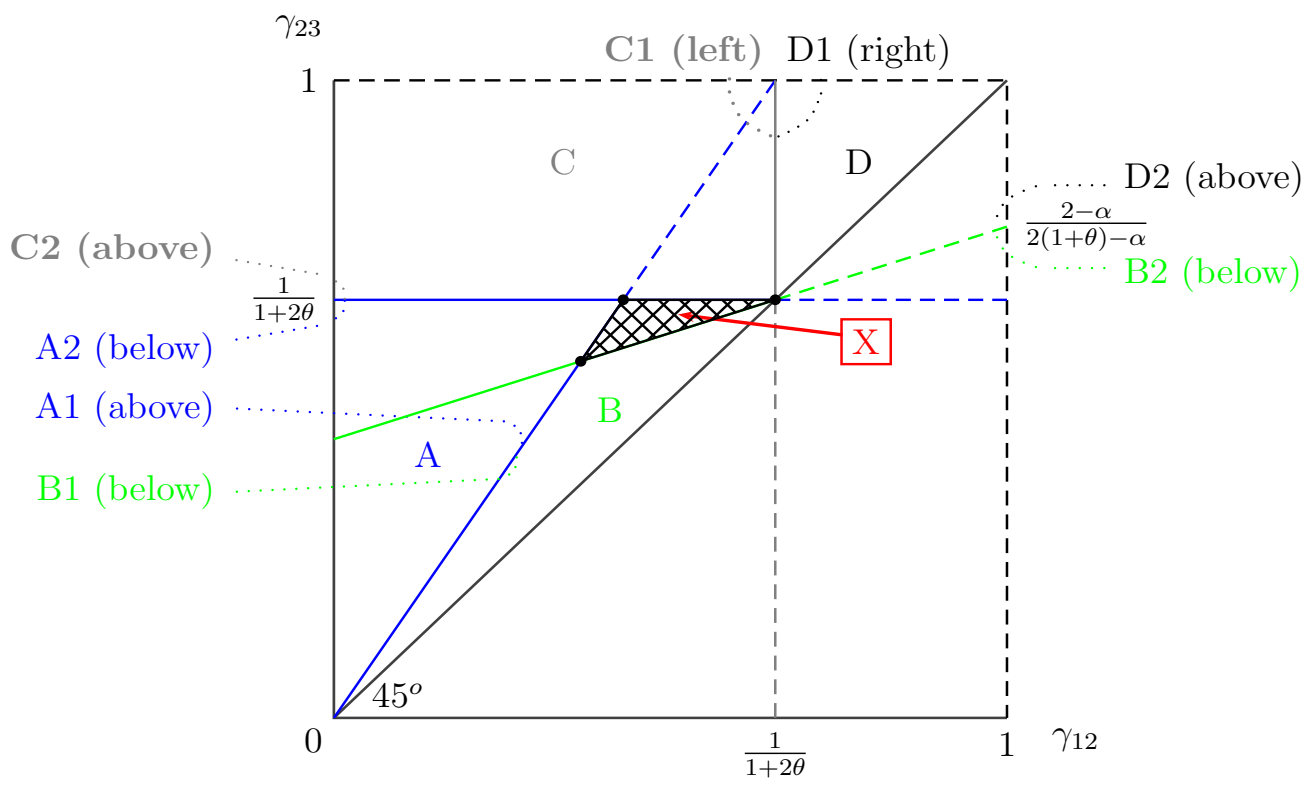

Figure A.12: Example of NPE outcomes for a range of $\gamma_{i}$

Recall that when $\theta=0$, only the outcome with the full set of treatments may exist and is neologism proof (see appendix A.3.1). This case would be shown with area A covering the entire upper quadrant of Figure A.12. The conditions for the other outcomes would not be satisfied, and areas $\mathrm{B}, \mathrm{C}$ and $\mathrm{D}$ would disappear. If $\theta$ were increased, however, area A would contract and some of the other areas would expand.

In this example, the figure is drawn for $\theta>0$ so that any of the four NPE outcomes can occur. For moderate values of $\gamma_{12}$ (about halfway between 0 and $\frac{1}{1+2 \theta}$ ), the equilibrium outcome may be $T_{0}=\left\{t_{1}, t_{2}, t_{3}\right\}$ (area A), $T_{0}=\left\{t_{1}, t_{3}\right\}$ (area B), $T_{0}=\left\{t_{2}, t_{3}\right\}$ (area C) or an equilibrium may not exist (area $\mathrm{X}$ ), depending on whether $\gamma_{23}$ is relatively low or high.

Suppose that both $\gamma_{12}$ and $\gamma_{23}$ were moderate. An increase in $\theta$ would see area A recede and the point corresponding to the moderate values of $\gamma_{12}$ and $\gamma_{23}$ could now fall in area for outcome B. In this outcome expected treatment costs may rise. This would be the result where sharper incentives counterproductively increase expected treatment costs. 
Next we consider the intuition for which outcome may occur when only one of the two equilibrium outcomes with $\left|T_{0}\right|=2$ exists. When $\gamma_{23}$ is very high, $P\left(\gamma_{23} \leq \pi\right)$ is relatively small. This creates a bias towards satisfying the conditions for $T_{0}=\left\{t_{1}, t_{2}\right\}$ (area $\mathrm{C}$ ). The intuition here is that there is a smaller chance that a patient would cost-effectively be treated with $t_{3}$ from the administrator's perspective, so omitting $t_{3}$ imposes a relatively small expected harm while saving on treatment costs. In contrast, when $\gamma_{23}$ is lower and $\gamma_{12}$ higher, $P\left(\pi \leq \gamma_{23}\right)$ is lower and $P\left(\gamma_{12} \leq \pi\right)$ is higher. This favours satisfying the conditions for $T_{0}=\left\{t_{1}, t_{3}\right\}$ (area B). Intuitively, there is a greater proportion of patients who could cost-effectively be treated with $t_{3}$ (or who would suffer relatively high harm if they did not receive $t_{3}$ ), but a smaller proportion of patients who would be cost-effectively served with $t_{2}$.

The figure has no overlapping areas. This indicates that the conditions for each outcome are mutually exclusive. Depending on the set of model parameters, however, it is possible that an equilibrium outcome does not exist. In this example, this possibility is represented by area $X$; this area is affected by the choice of $\alpha$. If $\alpha=1$, which implies that $\gamma_{13}=\gamma_{23}$, then constraints B2 and D2 collapse to A2 and C2. In this case, an equilibrium outcome exists for the entire range of $\theta$, but either $T_{0}=\left\{t_{1}, t_{3}\right\}$ (area B) exists or $T_{0}=\left\{t_{1}, t_{2}\right\}$ (area C) exists for intermediate values of $\gamma_{12}$ and $\gamma_{23}$. 


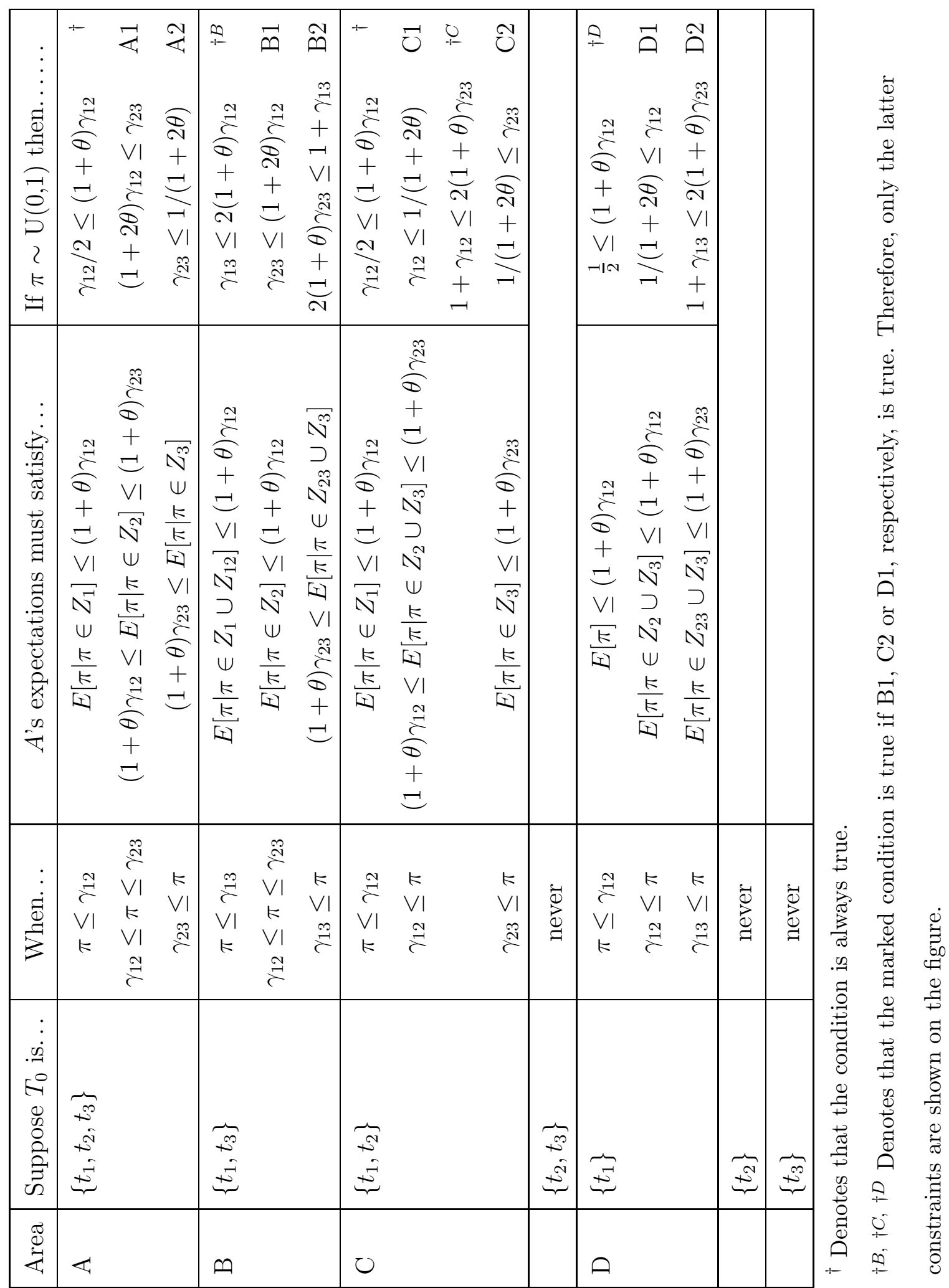

Table A.5: Conditions for $T_{0}$ to be consistent with an NPE outcome assuming $\pi \sim U(0,1)$ 


\section{A.4 Appendices to Chapter 5}

\section{A.4.1 Application of neologism-proofness with continuous choice variables}

This appendix discusses how the assumption used in section 5.3 departs from Farrell (1993). It shows that the result reached in that section is robust to adopting Farrell's (1993) assumption. It also motivates the assumption in section 5.3 by connecting the modelling result to the analysis of the CHE reforms.

Farrell's (1993) definition of a neologism requires that potential deviators strictly prefer that their message is believed. This condition is incorporated into his concept of credibility ${ }^{26}$ Farrell's (1993) credibility concept may be directly applied in section 5.3 where the specialist has a strict preference. That is, differences in the specialists' expected payoff under the incumbent administrator and her replacement influences the type of message he prefers to send. The consumer can factor this preference into how she interprets the specialist's message. We can use this to narrow down the possible equilibrium outcomes in each cheap-talk subgame.

The assumption used in section 5.3 differs slightly from Farrell's (1993) assumption. The model in section 5.3 considers cases where the specialist can be made indifferent between the incumbent administrator's reappointment and dismissal. This is a reflection of the wage being a continuous variable and the administrator wishing to minimise the wage for any particular outcome.

But Farrell's (1993) credibility concept treats boundary cases differently, where the sender's message is only credible when the sender strictly prefers the unanticipated alternative. The model in section 5.3 assumes that the specialist sends the administrator's preferred message when he is indifferent. This assumption may be interpreted as the specialist

\footnotetext{
${ }^{26}$ Farrell (1993):518-19 rules out randomisation over messages that have the same equilibrium meaning by assuming a message sender "has a slight preference for the truth".
} 
following some broader rule or norm of compliance within an employment relationship when he is otherwise indifferent.

The remainder of this note looks at whether the equilibrium outcomes in section 5.3 are robust to the application of Farrell's (1993) approach. In a pooling type equilibrium, credibility is not an issue. The consumer's optimism, that is, the belief that $\pi^{*} \leq \pi$, means she reappoints the administrator when uninformed. Therefore, the administrator does not need to pay the specialist to provide a credible message. Instead, the administrator can minimise the wage bill by paying the minimum possible wage $w_{B}$. In equilibrium, the specialist strictly prefers the incumbent to be replaced regardless of the quality level, so the specialist cannot use a neologism to disturb the putative equilibrium outcome.

In a separating type equilibrium, the consumer's default decision is to replace the incumbent if she does not receive a credible signal that quality is high, that is, the belief that $\pi<\pi^{*}$. Therefore, the administrator requires an informative signal if she is to have a chance of reappointment, and so the administrator offers $\underline{w}$. The specialist strictly prefers the administrator to be replaced when $q=q_{L}$ and $w=\underline{w}$, as $\underline{w}+q_{L}<w^{\prime}+q^{\prime}{ }^{27}$ When $q=q_{H}$ and $w=\underline{w}$ then the specialist is indifferent. This indifference flows through to the message the specialist prefers to send. Any message is equally as good as any other from the specialist's perspective when $q=q_{H}$, so he may randomly choose a message ${ }^{28}$ That is, the probability that the specialist would recommend reappointment when $q=q_{H}$ is $\mu \leq 1$

If the specialist uses a strictly mixed strategy, it would affect the informativeness of some of the specialist's messages. The consumer should find the specialist's recommendation to reappoint is credible, as the specialist would only it suggest it when $q=q_{H} \cdot{ }^{29}$ However, the consumer cannot draw such a clear interpretation from a recommendation to replace the

\footnotetext{
${ }^{27}$ This follows from the definition of threshold wage, $\underline{w}=w^{\prime}+q^{\prime}-q_{H}$.

${ }^{28} \mathrm{An}$ alternative way to think of the specialist's strategy is that the consumer is uncertain whether the specialist is telling the truth in a given instance, but she believes that the specialist tells the truth a certain proportion of the time.

${ }^{29}$ By Bayes' rule, $P\left(q=q_{H} \mid m \in m_{H}\right)=1$, and the consumer finds the specialist's message credible and her best choice is to concur with the specialist and reappoint the administrator.
} 
administrator. The specialist may send a message $m \in M_{L}$ when $q=q_{L}$ or as part of his randomised strategy when $q=q_{H}$. As a result, the specialist's potential indifference (and mixed strategy) erodes the informativeness of his message that quality is low (relative to using a pure strategy). In this scenario, the consumer remains uncertain of quality when the specialist indicates quality is low. As such, the consumer prefers to act on her prior beliefs that $\pi<\pi^{*}$ and she would replace the administrator ${ }^{30}$ This choice is consistent with the specialist's indifference and his consequent message. However, such an outcome is not sequentially rational for the administrator.

A wage offer of $w=\underline{w}$ is not the administrator's best response if it results in a risk of dismissal when $q=q_{H}$. A wage of $w=\underline{w}$ introduces such a risk if the specialist plays a strictly mixed strategy. The administrator could do better by offering a slightly higher wage, $w=\underline{w}+\epsilon$, so that the specialist would strictly prefer to recommend reappointing the administrator when $q=q_{H}{ }^{31}$ Then the consumer should find both types of recommendation credible as the specialist would only want to make a particular recommendation when it is in both their interests for the consumer to follow the specialist's advice. This alternative wage offer would guarantee the administrator's reappointment when $q=q_{H}$, rather than risk dismissal if the consumer remains uncertain because of the specialist's randomisation over his choice of message.

\footnotetext{
${ }^{30} \mathrm{~A}$ recommendation to replace, $m \in M_{L}$, leaves the consumer uncertain about whether the specialist is indifferent or strictly prefers the administrator to be replaced. But in this scenario, the consumer prefers to follow the specialist's recommendation to replace regardless of $\mu$. The probability that $P\left(q=q_{H} \mid m \in M_{L}\right)$ is $\frac{(1-\mu) \pi}{(1-\mu)(1-\pi)}$ by Bayes' rule. When $\mu=1$, then this probability equals zero. That is, as the specialist never indicates quality is low when it is high then the consumer believes there is a zero probability of high quality when the specialist recommends replacement. So the consumer prefers to follow the specialist's implicit recommendation. When $\mu=0$, then $P\left(q=q_{H} \mid m \in M_{L}\right)=\pi$. That is, if the specialist always prefers the administrator's replacement, despite quality being high, then the consumer is uncertain of what quality is when she hears $m \in M_{L}$, and she acts on her prior beliefs. A condition for the separating equilibrium is that the consumer's prior belief is that $\pi<\pi^{*}$. This implies she would prefer to dismiss the administrator without credible information that quality is high. So, regardless of how much the specialist mixes, the consumer prefers to replace the administrator on the specialist's recommendation as $P\left(q=q_{H} \mid m \in M_{L}\right) \leq \pi<\pi^{*}$.

${ }^{31}$ This applies as long as $\epsilon$ is not so great that the administrator does not wish to pay for a signal. I.e. $\mu \pi 2(G-w)+(1-\mu) \pi(G-w)+(1-\pi)(G-w)<\pi 2(G-w-\epsilon)+(1-\pi)(G-w-\epsilon) \Rightarrow \epsilon<\frac{(1-\mu) \pi(G-w)}{1+\pi}$. This condition imposes a restriction on the separating equilibrium, as for an $\epsilon$ above this threshold the administrator would prefer a pooling equilibrium outcome and she would prefer $w=w_{B}$.
} 
As the administrator wishes to minimise the wage, she would aim to make $\epsilon$ as small as possible in equilibrium. In the limit, as $\epsilon$ approaches zero, the wage approaches the putative separating equilibrium offer of $w=\underline{w}$. Here, the specialist plays a pure strategy where $\mu=1$, that is, the specialist always chooses $m \in M_{H}$ when $q=q_{H}$.

While there is a range of strategies the specialist may employ when indifferent, for example he may use a range of randomised strategies, the argument above rules out a mixed strategy for the specialist in equilibrium when $\pi<\pi^{*}$. That is, backwards induction suggests the proposed wage, $\underline{w}$, can be supported as a credible wage even when the specialist is indifferent. The assumption about how the specialist acts when indifferent does not upset the separating equilibrium characterised in section 5.3 .

Applying Farrell's (1993) approach with strict preference would still yield a unique equilibrium outcome of one of two types (where the type depends on $\pi$ ). The indifference assumption used in section 5.3 simplifies the analysis without substantially altering the equilibrium outcome (relative to using Farrell's (1993) approach). But the approach used here allows the administrator to hold the specialist to his reservation utility, allowing consideration of a separating equilibrium where the specialist is indifferent. 


\section{A.4.2 The minimum wage and the signalling wage}

This appendix provides a rationale for the simplifying assumptions used in Chapter 5 that:

- the minimum wage the administrator offers the specialist, $w_{B}$, is sufficient to induce the specialist's participation

- the signalling wage is greater than the minimum wage $\underline{w}>w_{B}$.

\section{Reservation wages and the participation constraint}

The specialist's reservation wage will depend on the anticipated equilibrium, as his payoff depends on what happens in the the second stage. We focus on the three potential PBE outcomes identified in Chapter 5: a separating outcome $(S)$, a pooling outcome where the administrator's tenure is always terminated $(T)$, and a pooling outcome with reappointment $(R)$. We denote the reservation wage of the specialist in each of these three equilibrium candidates as $w_{S}, w_{T}$ and $w_{R}$.

If the specialist accepts the administrator's offer, the specialist's expected payoff of $w+$ $E[q]$, in the first stage and the expected payoff in the second stage as tabulated below.

\begin{tabular}{|l|c|c|c|}
\hline $\begin{array}{c}\text { With } \\
Y_{0} \ldots\end{array}$ & $\begin{array}{c}\text { the type of } \\
\text { equilibrium is } \ldots\end{array}$ & with the result. . & $\begin{array}{c}\text { giving } S \text { an expected payoff } \\
\text { in the second stage of } \ldots\end{array}$ \\
\hline$\{T, R\}$ & separating & $\begin{array}{c}T \text { with probability }(1-\pi) \\
R \text { with probability } \pi\end{array}$ & $\begin{array}{c}(1-\pi)\left(w^{\prime}+q^{\prime}\right) \\
+\pi\left(w_{S}+q_{H}\right)\end{array}$ \\
\hline$\{T\}$ & pooling & $T$ & $w^{\prime}+q^{\prime}$ \\
\hline$\{R\}$ & pooling & $R$ & $w_{R}+E[q]$ \\
\hline
\end{tabular}

Table A.6: Potential PBE outcomes and the specialist's second stage payoff 
Let $\breve{w}+\breve{q}$ be the payoff of the specialist per stage in his best alterative job. Taking the the payoffs in each stage, we can characterise the specialist's participation constraint in each of the three outcomes:

$$
\begin{aligned}
& S: 2(\breve{w}+\breve{q})=\left(w_{S}+E[q]\right)+\left((1-\pi)\left(w^{\prime}+q^{\prime}\right)+\pi\left(w_{S}+q_{H}\right)\right) \\
& T: 2(\breve{w}+\breve{q})=\left(w_{T}+E[q]\right)+\left(w^{\prime}+q^{\prime}\right) \\
& R: 2(\breve{w}+\breve{q})=2\left(w_{R}+E[q]\right)
\end{aligned}
$$

We can solve for the reservation wage in each outcome. For example:

$$
w_{R}=\breve{w}+\breve{q}-E[q]
$$

\section{Reservation wages and the signalling cost}

Chapter 5 concludes that there is a unique NPE outcome, that might be one of two types: a separating equilibrium $(S)$ or a pooling equilibrium where the administrator is always reappointed $(R)$. We focus on these two types of NPE and examine the cost to the administrator when the signal is off and of turning the signal on.

\section{Optimism}

In the outcome where the consumer is optimistic, that is $\pi^{*}<\pi$, and $Y_{0}=\{R\}$, the administrator is always reappointed. In this outcome, the specialist is offered his reservation wage $w_{B}$, which we assumed was less than $\underline{w}$. Below we identify a condition for the specialist to be willing to accept such a wage, given he anticipates that the administrator will always be reappointed.

Equation A.20a gives the specialist's reservation wage in this outcome. For $w_{B}<\underline{w}$ to be true, we require that $\breve{w}+\breve{q}<w+E[q]$ for some $w<\underline{w}$. As the signalling wage is $\underline{w}=w^{\prime}+q^{\prime}-E[q]$ and $E[q]=\pi q_{H}+(1-\pi) q_{L}$, we may express this condition as:

$$
\breve{w}+\breve{q}<w^{\prime}+q^{\prime}-(1-\pi)\left(q_{H}-q_{L}\right) .
$$




\section{Pessimism}

In the outcome where the consumer is pessimistic, that is $\pi<\pi^{*}$, and $Y_{0}=\{T, R\}$, the administrator is dismissed if quality is low and reappointed if quality is high. In this outcome, the administrator has to pay the specialist the higher wage of $\underline{w}$.

The condition for the specialist to accept an offer of $w=\underline{w}$ in this outcome, from equation A.20c and noting the stage two payoffs in Table A.6, is:

$$
2(\breve{w}+\breve{q})<(\underline{w}+E[q])+(1-\pi)\left(w^{\prime}+q^{\prime}\right)+\pi\left(\underline{w}+q_{H}\right)
$$

As the signalling wage is $\underline{w}=w^{\prime}+q^{\prime}-E[q]$ and $E[q]=\pi q_{H}+(1-\pi) q_{L}$, we can simplify condition A.23 to:

$$
\breve{w}+\breve{q}<w^{\prime}+q^{\prime}-\frac{1-\pi}{2}\left(q_{H}-q_{L}\right)
$$

Condition (A.24) is weaker than A.22). Therefore, we need only assume A.22 to justify the simplifying assumption used in section 5.3 that $w_{B}<\underline{w}$. 
